\title{
Review \\ Carbon Nanomaterials: Synthesis, Functionalization and Sensing Applications
}

\author{
Giorgio Speranza ${ }^{1,2,3}$ (D)
}

check for

updates

Citation: Speranza, G. Carbon

Nanomaterials: Synthesis,

Functionalization and Sensing

Applications. Nanomaterials 2021, 11,

967. https://doi.org/10.3390/

nano11040967

Academic Editor: Alexander Kromka

Received: 16 February 2021

Accepted: 17 March 2021

Published: 9 April 2021

Publisher's Note: MDPI stays neutral with regard to jurisdictional claims in published maps and institutional affiliations.

Copyright: (C) 2021 by the author. Licensee MDPI, Basel, Switzerland. This article is an open access article distributed under the terms and conditions of the Creative Commons Attribution (CC BY) license (https:/ / creativecommons.org/licenses/by/ $4.0 /)$.

\author{
CMM-FBK, v. Sommarive 18, 38123 Trento, Italy; speranza@fbk.eu \\ IFN_CNR, CSMFO Lab., via alla Cascata 56/C Povo, 38123 Trento, Italy \\ Department of Industrial Engineering, University of Trento, v. Sommarive 9, 38123 Trento, Italy
}

\begin{abstract}
Recent advances in nanomaterial design and synthesis has resulted in robust sensing systems that display superior analytical performance. The use of nanomaterials within sensors has accelerated new routes and opportunities for the detection of analytes or target molecules. Among others, carbon-based sensors have reported biocompatibility, better sensitivity, better selectivity and lower limits of detection to reveal a wide range of organic and inorganic molecules. Carbon nanomaterials are among the most extensively studied materials because of their unique properties spanning from the high specific surface area, high carrier mobility, high electrical conductivity, flexibility, and optical transparency fostering their use in sensing applications. In this paper, a comprehensive review has been made to cover recent developments in the field of carbon-based nanomaterials for sensing applications. The review describes nanomaterials like fullerenes, carbon onions, carbon quantum dots, nanodiamonds, carbon nanotubes, and graphene. Synthesis of these nanostructures has been discussed along with their functionalization methods. The recent application of all these nanomaterials in sensing applications has been highlighted for the principal applicative field and the future prospects and possibilities have been outlined.
\end{abstract}

Keywords: carbon nanostructures; fullerenes; nano onions; quantum dots; nanodiamonds; carbon nanotubes; graphene

\section{Contents}

1. Introduction

2. Properties of Carbon Nanostructures

3. Fullerenes and Carbon Onions

3.1. Fullerene Synthesis

3.2. Fullerene Functionalization

3.3. Carbon Onion Synthesis

3.4. Carbon Nano-Onion Functionalization

3.5. Fullerenes Sensing

3.6. Carbon Nano-Onion Sensing

4. Nanodiamonds

4.1. Nanodiamond Synthesis

4.2. Nanodiamond Functionalization

4.3. Nanodiamond Sensing

5. Carbon Quantum Dots

5.1. Carbon Quantum Dot Synthesis

5.2. Carbon Quantum Dot Functionalization

5.3. Carbon Dots Sensing

6. Carbon Nanotubes

6.1. Carbon Nanotube Synthesis 
6.2. Carbon Nanotube Functionalization

6.3. Carbon Nanotube Sensing

7. Graphene

7.1. Graphene Synthesis and Functionalization

7.1.1. Bottom-Up Synthesis

7.1.2. Top-Down Synthesis

7.2. Graphene Functionalization

7.2.1. Covalent Functionalization

7.2.2. Non-Covalent Functionalization

7.3. Graphene Sensing

8. Conclusions

\section{Introduction}

Novel technological solutions are needed to face emerging global challenges such as environmental pollution, massive energy production, need of additional agricultural and food production. To increase the productivity while reducing the generation of pollutants, new advanced systems are needed to improve and automate operations which autonomously can monitor infrastructures, the environment, and the processes efficiencies [1-8]. In this respect, the potentialities enabled by sensing technologies are considerable.

The global gas sensor market size was estimated at USD 2.33 billion in 2020 and is expected to reach USD 2.50 billion in 2021. There is growing request of sensing power to monitor the environment, indoor areas, the health conditions, the industrial processes, the components in operando conditions (see applications in automotive), and sparse controls in smart cities. In this respect, the electrochemical segment dominated the market in 2020 and accounted for a share of more than $21.0 \%$ of the global revenue [9].

Nowadays there is broad class of sensors for checking gas molecules, heavy metals, humidity, recognize biomolecules, toxic substances, $\mathrm{pH}$, pressure, and many others [10]. However, most of them display a non-ideal limit of detection (LOD), scarce sensitivity and/or selectivity, have slow responses, need pretreatment and are expensive. In this panorama nanomaterials can play a crucial role because they offer a solution to the limitations of conventional systems providing important advances in the material properties [10].

A revolution in material science occurred with the recognition that reduction in the nanorange of at least one of the system dimensions introduces novel unusual properties. In this respect, nanomaterials open the perspective to improve parameters such as the sensibility and the reliability, make the response and recovery times shorter, open the possibility to perform in situ analysis, and their cost is low. All these properties are required for producing sensor devices.

Nanomaterials are attractive for a number of different applications, including energy production, biomedical applications, environmental protection, information technology, food, agriculture and many others. Then, significant efforts have been recently devoted for both the mass production of structurally homogeneous nanomaterials with well controlled surfaces and interfaces and their assembly into device architectures. In this scenario, the carbon-based nanomaterials have become one of the dominating materials in several sensor applications. A literature survey reveals that more than 2200 publications are related to nanomaterial and sensing and roughly about $50 \%$ of them regard carbon nanomaterials [11].

Among the various chemical elements, carbon plays a very special role. It provides the basis for the life in nature, it displays different orbital hybridization leading to the ability to generate different chemical bonds with different orientations. For this reason, carbon possesses different allotropic forms (graphite and diamond) and has the capability to generate a list of nanostructures namely graphene single sheets, mono and multiwalled carbon nanotubes, carbon fibers, fullerenes, onions, and nanodiamonds. In addition, carbon is able to bind to nearly all chemical elements generating an unlimited variety of molecules and compounds [12] possessing a number of different chemical and physical properties. Typically the size of carbon 
nanomaterials ranges from 1 to $100 \mathrm{~nm}$ and leads to significant changes with respect to the bulk counterparts namely the increased surface to volume ratio, the nanostructure shapes, the different chemical reactivity, the different optical properties ... [13].

As a consequence, carbon nanomaterials are widely utilized in many sectors. They are used in environmental applications for water treatment $[14,15]$, and other separation processes [16,17], for environment remediation [18-20]; in electronics where they have shown remarkable utility for the excellent electrical [21,22] and optical properties [23-25]. This, combined with the molecular sized diameter and microscopic structure enable the development of novel electronic devices [26]. The high mechanical strength, electrical and thermal conductivity [27-29] make them ideal as reinforcing elements [30-32], as protective materials [33-35] and to make conductive polymers [36,37]. Carbon nanomaterials find application also in the biomedical field for sensing applications and in controlled pharmaceuticals and drugs delivery [38-42].

As mentioned before, carbon (C) atoms are able to organize themselves in different structures as depicted in Figure 1. When $\mathrm{C}$ atoms are arranged in a honeycomb lattice, they form the graphite crystal, a stack of two-dimensional single sheets. The single graphite layer constitutes the graphene [43] atomic crystal. Graphene nanostructures (GNs) were firstly isolated by the Nobel-prizes Geim and Novoselov in 2010. Another carbon-based structure is carbon nanotube (CNT). Discovered by Iijima in 1991, it may be regarded as a single graphene layer rolled along an axis aligned along the graphene crystalline directions [44]. As CNTs also carbon fibers (CFs) are unidimensional system. However, CFs are disordered, tangled structures possessing a two-dimensional long-range order of C atoms organized in planar hexagonal networks while in the direction orthogonal to these planes, CFs display only a short range order due to parallel plane stacking [45].

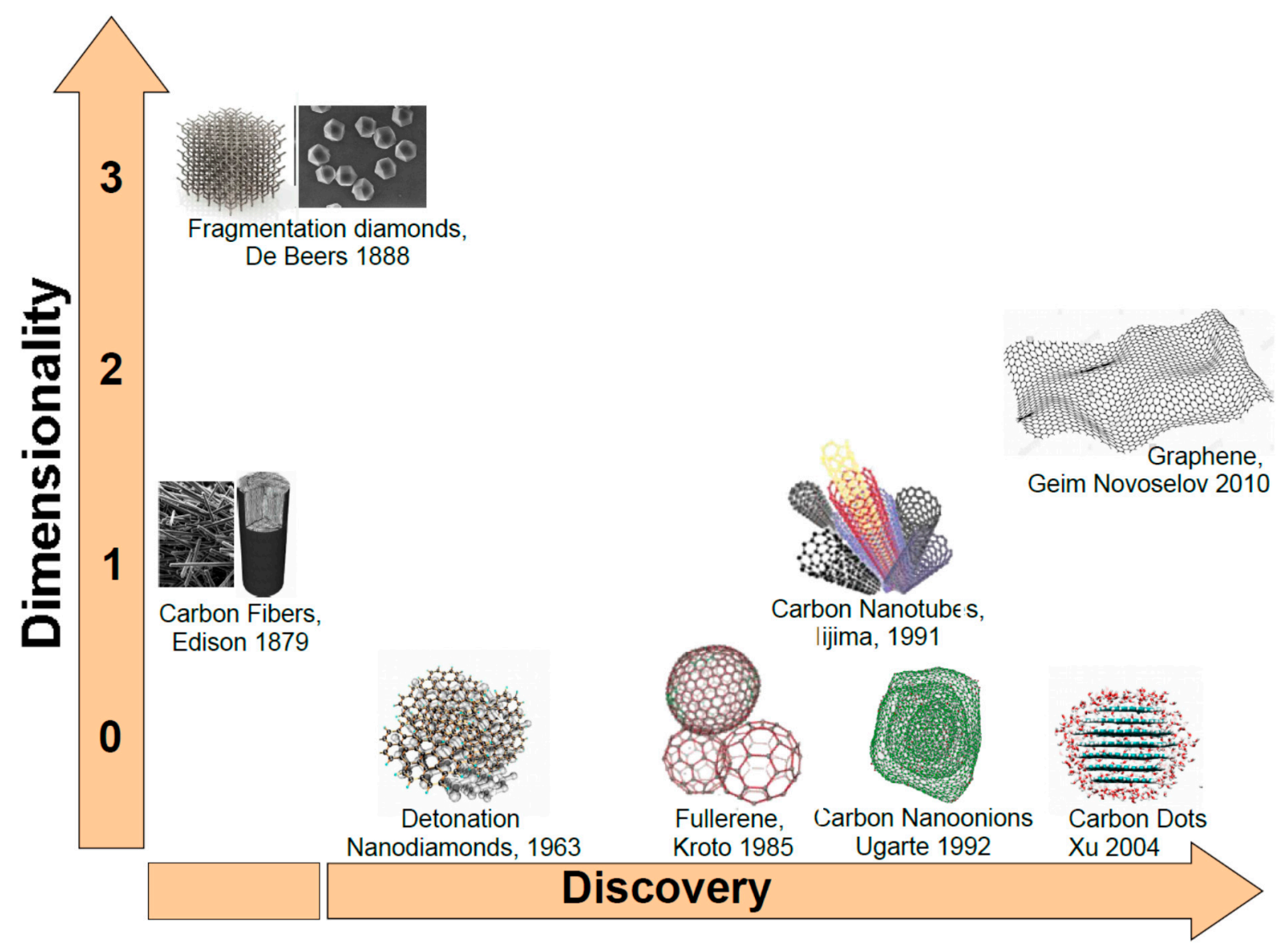

Figure 1. Carbon nanostructures ordered following dimensionality and discovery time. 
If in unidimensional carbon structures we reduce their length to the nanometer size we will obtain nanocages. Fullerenes discovered by Kroto and Smalley in 1985 is a perfectly spherical nanocage formed by a number of pentagonal and hexagonal rings [46]. Carbon nano-onions (CNOs) are cages with spherical or polyhedral shape formed by several fullerene-like overlapped carbon shells which are defective and disordered to a certain degree [47]. They were discovered in 1992 by Ugarte during electron beam irradiation of an amorphous carbon sample using a TEM microscope [48]. Another unidimensional carbon nanostructure (CNS) is represented by the carbon dots (CDs). CDs are nanoparticulate where graphitic and amorphous carbon phases coexist. Typically, the average dimension of carbon dots is about $5 \mathrm{~nm}$. Quantum confinement effects induce excellent optical properties as highly tunable photoluminescence (PL), high photostability easy functionalization of their surface and biocompatibility [49] make them good competitor of quantum dots based on toxic chemical elements such as cadmium. Another CNS with excellent degree of biocompatibility is the nanodiamond. Nanodiamonds (NDs) were discovered in the sixties [50]. Depending on the synthesis process, NDs dimensions are in the 5-100 nm range. In NDs, $\mathrm{C}$ atoms are $\mathrm{sp}^{3}$ hybridized orbitals [51] leading to the formation of the hexagonal or cubic diamond lattices. NDs possess distinctive electronic and optical properties deriving from dopants (N, Si, Ge ... ) present in the structure as defects [52].

This review complements previous works on this topic giving a complete description of carbon-based nanostructure's properties, of their synthesis and functionalization processes. For each of these nanostructures, an extensive survey of the sensing applications is also provided together with an accurate summary of the detection modalities, the kind of surface chemistry and the analyte sensed to facilitate the consultation and retrieve the information sources.

\section{Properties of Carbon Nanostructures}

Carbon is a unique element of the periodic table possessing the extraordinary capability to organize its four valence electrons in different hybridization states, namely sp, sp2, sp3 leading to both strong covalent and weak $\pi-\pi$ bonds. The different hybridizations enable C-atoms to assume different allotropic forms with diamond and graphite as main prototypes and to form a wide range of structures, from small molecules to long chains [53]. The different organization of C-atoms in the crystalline lattice of graphite or diamond is also accompanied by rather different physical properties. Graphite is a stack of weakly bonded single layers where carbon atoms are organized in a honeycomb structure. Because the weak van der Waals interaction between layers, the $\pi$ electrons are quasi-free thus leading to the semimetallic character of graphite [54]. The valence and conduction bands are overlapped in a point thus leading to a zero optical gap. As a consequence, the graphite has a dark aspect with high absorption coefficient. Different is the case of diamond where the $\mathrm{sp}^{3}$ hybridization generates four strong covalent bonds oriented along the axis of a tetrahedron and to a face centered Bravais lattice. In this case mobility of the electrons is absent and diamond is a highly insulating material characterized by an optical gap as high as $5.5 \mathrm{eV}$. The high optical gap makes pure diamond one of the most broadly transmitting of all materials [55]. It is transparent over a wide optical range extended well out from the visible regions (observe that absorption lines are present due to impurities mainly $N, B, H$, $\mathrm{Ni}$... ). Its transmission spectrum shows a flat featureless window for wavelengths longer than $\sim 225 \mathrm{~nm}$ and moderate absorption in the range 2.6 to $6.2 \mu \mathrm{m}$ due to multiphonon processes [55].

Apart from these two representative forms, carbon can also organize in amorphous structures. However, some short-range order can be observed in amorphous carbon $(\mathrm{aC})$ phases. In aC both graphitic $\mathrm{sp}^{2}$ and tetrahedral $\mathrm{sp}^{3}$ hybrids coexist. The prevalence of one or the other of these hybrids imparts properties mirroring those of graphite or diamond. In the first case the amorphous carbon has poor mechanical properties, high extinction coefficient. Differently, in diamond-like-carbons and in highly tetrahedral amorphous carbon are very hard and the optical gap can be increased till to $4.5 \mathrm{eV}$ with correspondent 
high transparency. Presence of hydrogen can also modulate the aC properties leading to both hard and polymer-like structures which can be interesting as biomaterials. Amorphous phases can be present in CNS or amorphous carbon nanoparticles can be produced [56-59] for their optical [60], electrical [61], mechanical properties [62].

As in the macroscopic allotropes, also the properties of a given CNS depend on the type of hybridization assumed by carbon atoms in the nanostructure. However, due to the quantum confinement imposed by the nanometric dimension or on structural arrangements as in fullerenes some differences appear. Considering the applicative point of view, the transduction of a given physical entity (generally an electrical signal) is made by the integration of a CNS into a microsystem which is used in the development of electronic, photonic, and optoelectronic devices. The selection of a given nanostructure depends on the signal to transduce and on the physicochemical properties of the CNS. In the following we will consider the properties of the single carbon nanoallotropes and the relative applications which are summarized in Figure 2.

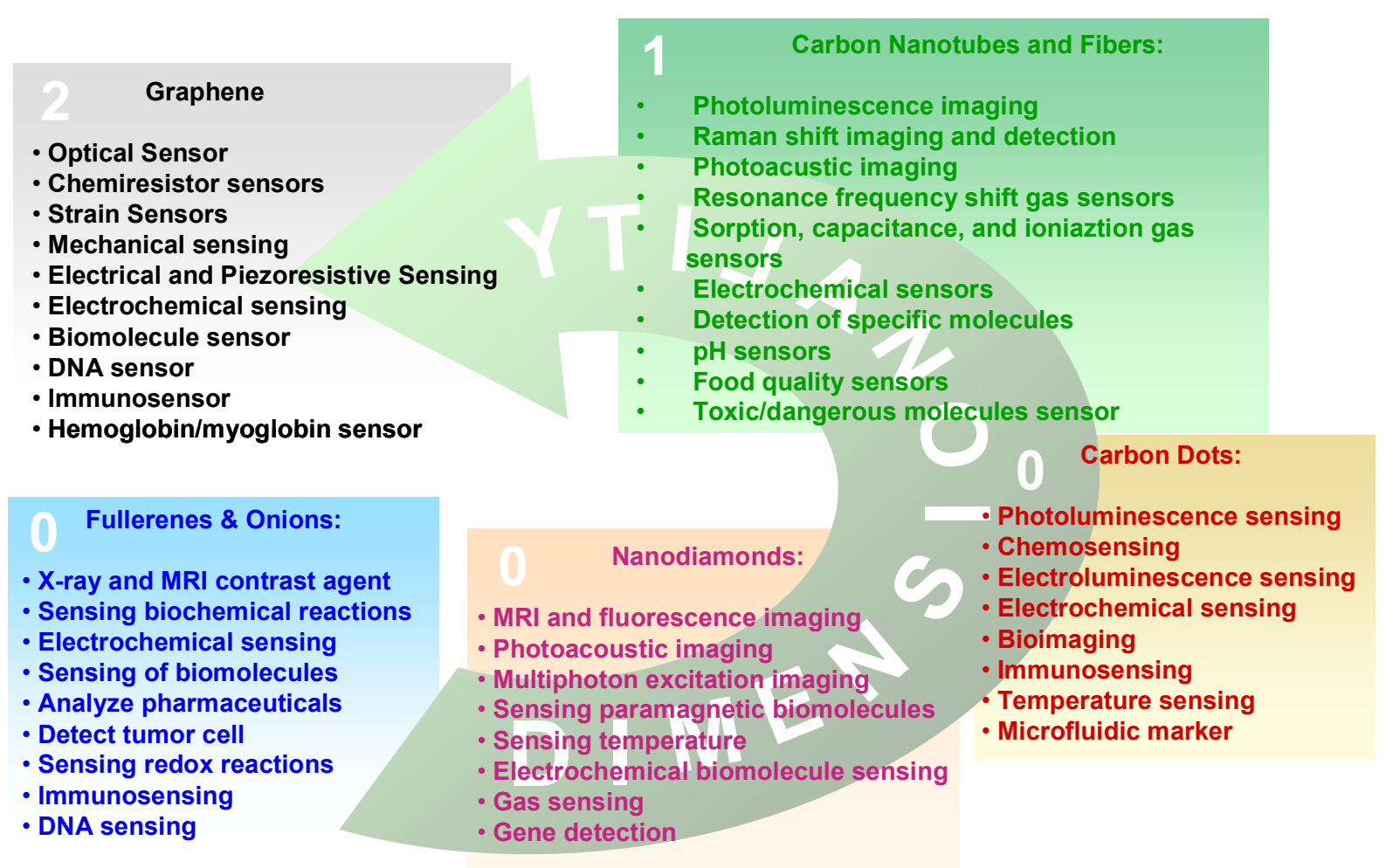

Figure 2. Carbon nanoallotropes and the relative sensing applications.

\section{Fullerenes and Carbon Onions}

As seen, fullerenes are closed hollow cages in which C-atoms are $\mathrm{sp}^{2}$ - hybridized carbon atoms. However, to generate the spherical cage, the structure cannot be generated using only hexagonal rings. Following Euler's theorem, it is possible to show that a spherical surface must contain exactly 12 pentagons. Fullerenes may be regarded as a class of closed-cage carbon molecules, $C_{n}$, where $n$ indicates the total number of carbon atoms forming 12 pentagons and a variable number of hexagons. Depending on the number of hexagons, fullerenes of different sizes are obtained. The number $n$ may assume values $n=20$ and $n=20+2 \mathrm{k}(\mathrm{k}=1,2,3 \ldots)$. A fullerene containing $22 \mathrm{C}$-atoms does not exist. The different dimensions and shapes of fullerenes have significant effects on their optical and chemical properties. For example the absorption spectra of the fullerene depend on the number of atoms $n$ as pictorially showed in Figure 3 where the color changes with $n$. Fullerenes display a hydrophobic trait and the solubility decreases with increasing their size [63]. For their aromatic character fullerenes are generally soluble in organic 
hydrocarbons and halogenated solvent [64]. The dimension of fullerene structure affects also its reactivity. In addition, one of the important driving forces is the relief of strain affecting the cage structure, enabling the return to $\mathrm{sp}^{3}$ hybridization. Then the chemical reactivity of fullerenes increases as their size increases resulting in a reduced curvature and strain towards a more graphitic-like planar surface [65].

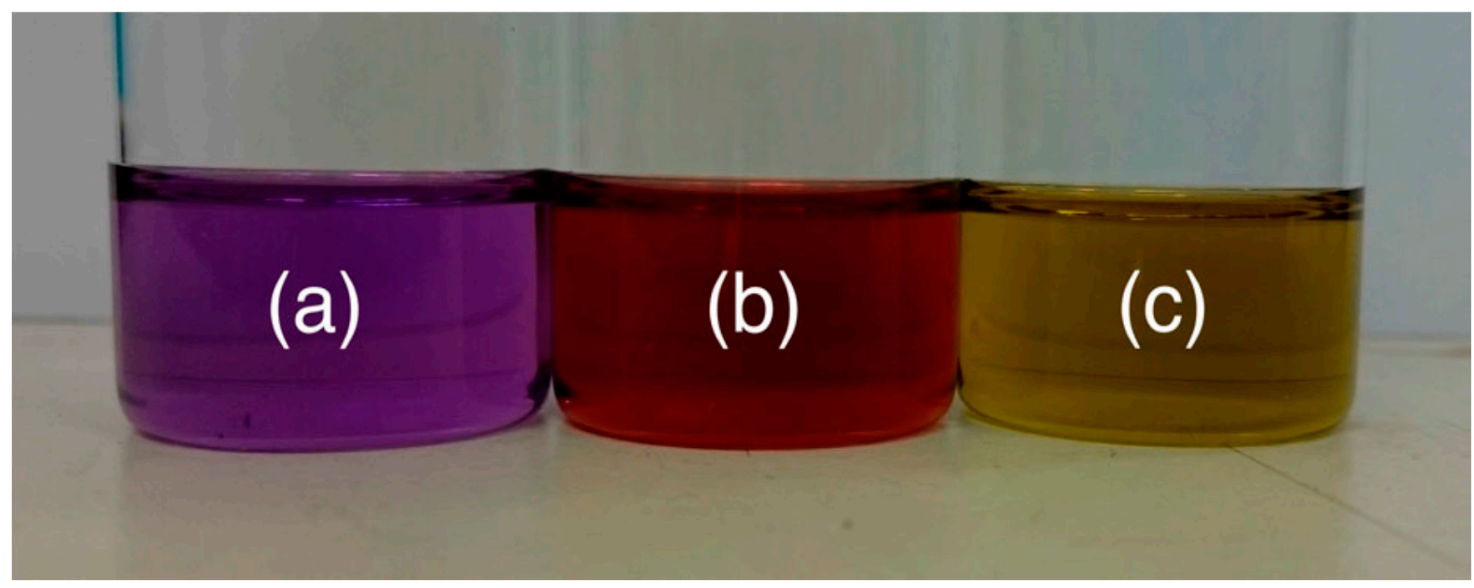

Figure 3. The absorption spectra of the fullerenes change as the size of the conjugated system increases: with slight variations depending on solvent, solutions of $\mathrm{C}_{60}$ are an intense purple color (a), $\mathrm{C}_{70}$ red like wine (b) and $\mathrm{C}_{84}$ a green-yellow (c) (all solutions are in toluene). Generally speaking, the gap between the highest occupied molecular orbital and the lowest unoccupied molecular orbital decreases with increasing cage size leading to optical absorptions of lower energy, i.e., longer wavelength. Reprinted with permission from [65].

\subsection{Fullerene Synthesis}

$\mathrm{C}_{60}$ is the more common among the fullerenes and the most widely studied. However, despite numerous attempts of explanation, still the mechanism of fullerene formation is unknown. The common methods to produce $\mathrm{C}_{60}$ vaporization of graphite electrodes using arc or plasma discharges [66-68] or using laser ablation [46,66,69], pyrolysis of hydrocarbons [70-72]. This process was optimized for mass production by Murayama [73] leading to a soluble mixture of $60 \% \mathrm{C}_{60}, 25 \% \mathrm{C}_{70}$ and a remaining $15 \%$ composed by bigger fullerenes up to $\mathrm{C}_{96}$. Initially the fullerenes are separated from soot using solvents as benzene or toluene. The remaining product is processed using column or liquid chromatography to separate fullerenes of different sizes [74].

Concerning carbon-onions (COs), these structures were obtained irradiating CNTs using an electron beam [75]. A modification of this method introduces gold nanoparticles promoting the formation of the nanoparticles with less extreme irradiation conditions [76] Bigger amounts of COs are produced by heating carbon soot at $2100-2250{ }^{\circ} \mathrm{C}$ under vacuum [77]. The nanostructures obtained with this method generally have a non-spherical shape and are formed by 2-8 shells with diameters of 3-10 $\mathrm{nm}$. Using arc discharge in water it is possible to obtain bigger spherical COs with a $25-30 \mathrm{~nm}$ diameter $[78,79]$. More recent synthesis process are based on solution ozonolysis [80] or by electrochemical processes [81].

$\mathrm{C}_{60}$ has an external diameter of $0.71 \mathrm{~nm}$, and its chemical properties are very similar to those of an organic molecule. However, the $\mathrm{C}_{60}$ molecule is considered to be not superaromatic due to the presence of pentagons where $\mathrm{C}$-atoms have the tendency to avoid formation of double bonds. In particular it is shown in literature that fullerene the $\mathrm{C}_{60}$ molecule will undergo a facile reduction, and because of the surface curvature of the surface, fullerene hybridization falls between $\mathrm{sp}^{2}$ and $\mathrm{sp}^{3}$. This together with topology account for the extraordinary ability of $\mathrm{C}_{60}$ to accept electrons [82]. This characteristic has a peculiar influence on the fullerene chemical reactivity and electrochemical properties and the large number of fullerene functionalization reactions that can be made on fullerenes. 


\subsection{Fullerene Functionalization}

The list of possible chemical reactions utilized to functionalize fullerenes is very long. Here we will present a selection of the most important reactions which are reviewed in [65,83-85]. Surface functionalization is a convenient route making fullerene soluble in both water and organic solvents $[86,87]$. Chemical modification of the fullerene surface may be performed following two different methods: (i) complexation with solubilizing agent to partially hide the fullerene hydrophobic surface [88]; (ii) covalent functionalization of the fullerene surface [89]. Oxygen based functional groups, mainly hydroxyl groups, may be grafted on the fullerene surface using strong acids at high temperature [90].

$C_{60}$ and $C_{70}$ fullerenes were mixed with an aqueous solution of nitric and sulphuric acids at a temperature of $85-115^{\circ} \mathrm{C}$. The acid induced oxidation of the fullerenes surfaces and to a grafting of about 15 hydroxyl groups/fullerene in average. Another possible reaction to form $\mathrm{OH}$ functionalized fullerenes (fullerenols) can be performed also adding strong basic $\mathrm{NaOH}$ to $\mathrm{C}_{60}$ benzene solutions in presence of tetrabutylammonium hydroxide acting as a catalyzer [91]. The reaction is more efficient than that based on strong acids resulting in a higher number of grafted hydroxyl groups. In another work, a drop of liquid $\mathrm{Na} / \mathrm{K}$ alloy was added to a suspension of $\mathrm{C}_{60}$ in tetrahydrofuran and a subsequent reaction of this intermediate with $\mathrm{O}_{2}$ and water [92]. The reaction is very efficient and produced an extended hydroxylation of the fullerenes, inducing hydrophilicity among the highest ever reported in literature. These reactions are broadly used to make fullerenes hydrophilic despite the lack of control on the density of addends. An alternative method to produce highly polyhydroxylated fullerenes is based on the use of a suspension of partially hydroxylated fullerenes and hydrogen peroxide at a temperature of $60^{\circ} \mathrm{C}$ [93]. The reaction is rather slow (4 days) but results in fullerenols with 36-40 hydroxyl groups in average, with 8-9 secondary bound water molecules. The product exhibited a high water solubility up to $58.9 \mathrm{mg} / \mathrm{mL}$ at $\mathrm{pH}=7$. Description of other methods of fullerenol synthesis may be found in [94-98].

Grafting of amine groups is another common functionalization process performed mixing $\mathrm{C}_{60}$ with different aliphatic primary amines as n-propylamine, $\mathrm{t}$-butylamine, and dodecylamine [99]. In other reactions, smaller primary, secondary amine chains as methylamine, diethylamine or ethylenediamine (as shown in Figure $4 \mathrm{~A}$ ) are reacted with $\mathrm{C}_{60}, \mathrm{C}_{70}$ fullerenes [100]. Another popular amination reaction is the 1,3-dipolar cycloaddition of an azomethine ylide to a $\mathrm{C}_{60}$ molecule (Prato reaction, see Figure $4 \mathrm{~B}$ ) produces a stable compound grafting a pyrollidine ring to $\mathrm{C}_{60}$ [101]. The beauty of this reaction is the possibility to introduce different functional groups to the fullerene moiety. Another possible reaction for the generation of well-defined amine addition products utilizes a modest excess of secondary diamines like $\mathrm{N}, \mathrm{N}^{\prime}$-dimethylethylenediamine or piperazine resulting in both monoand bisadducts with an overall $50-85 \%$ yield [102]. Addition of amine to $C_{60}$ can take place at room or high temperature involving electron transfer process [103]. In [104] diamine were added to $\mathrm{C}_{60}$ at low temperature. The reaction was performed to prepare fullerene diamine adduct which was the starting material for the synthesis of $\mathrm{C}_{60}$-polyamides. Amination was performed adding diamine, 1,4-Butanediamine, 1,6-Hexanediamine, to a benzene solution of $\mathrm{C}_{60}$ and diamine in benzene in appropriate proportions. The reaction led to the formation of the desired adducts in $80-89 \%$ yields. Formation of Penta- and Hexaamino- [60] fullerenes was obtained in presence of oxygen and secondary amines such as piperidine and $\mathrm{N}$-methylbenzylamine and $\mathrm{C}_{60}$ fullerenes [105]. A solvent-free chemical modification of $\mathrm{C}_{60}$ fullerene surface was carried out using amino terminated polyethylene glycol [106]. Authors studied the effect of the stirring rate and temperature on the $C_{60}$ modification yield. 
(A)

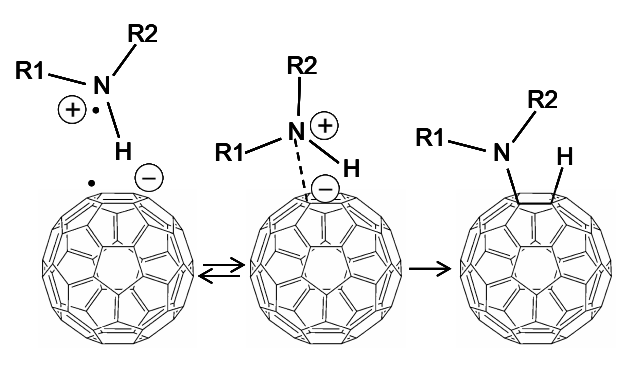

(B)<smiles>[R3]C(=O)C([R2])N([R])[C@H]([R2])C(=O)O</smiles>

(C)

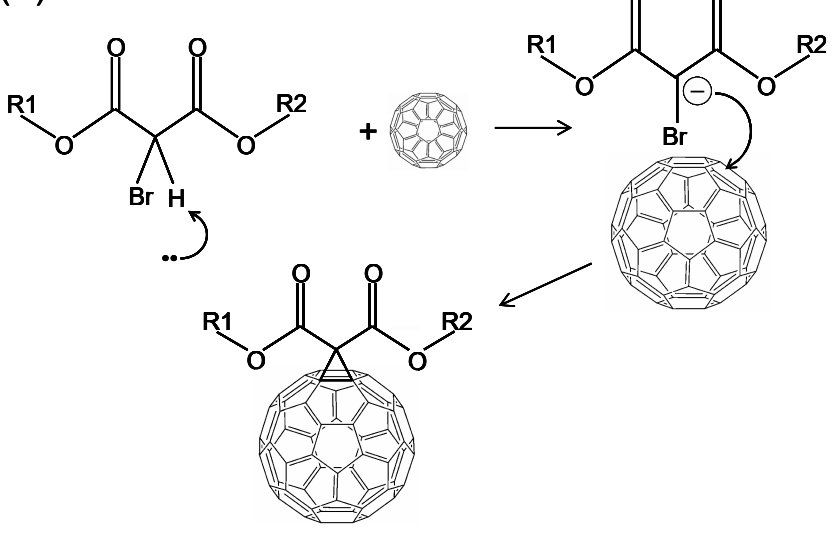

Figure 4. (A) Amination mechanism of $\mathrm{C}_{60}$. The first step is single-electron transfer to produce the $\mathrm{C}_{60}$ anion radical. Then radical recombination gives a zwitterion which can be stabilized by proton transfer to give the final product. (B) Prato reaction mechanism. The nitrogen lone pair of the amino acid attacks the aldehyde/ketone's polar carbonyl group and leads to the expulsion of water, before decarboxylation gives the reactive ylide intermediate. (C) Bingel reaction mechanism. Deprotonation by the strong base gives the nucleophilic malonate anion which then attacks the $[6,6]$ bond, $\mathrm{Br}$ is then expelled.

As a result, the $65 \%$ of $\mathrm{C}_{60}$ was modified at $60^{\circ}$ after $24 \mathrm{~h}$ stirring. Amination was also utilized to produce transparent fullerene containing sol-gel glasses. Fullerene amination was performed at $100{ }^{\circ} \mathrm{C}$ under nitrogen by reacting $\mathrm{C}_{60}$ with 6-amino-1-hexanol, cyclohexylamine, 2-(2-aminoethoxy)ethanol, and 3-aminopropyltriethoxysilane [107]. After filtration and purification, the authors obtained a yield from $32 \%$ to $82 \%$. A review on the reaction of between aliphatic amines and [60] fullerene may be found in [108].

The Bingel reaction is widely utilized for fullerene exohedral functionalization (see Figure 4C). This reaction involves the addition of an $\alpha$-halo ester/ketone to the cage under strongly basic conditions resulting in a methanofullerene [109]. There are several examples of the application of the Bingel reaction to modify the chemistry of the fullerene surface. For example, the Bingel reaction was used to synthesize $\mathrm{C}_{60}$ Hexakis-adducts [110]. Authors found that the original Bingel-Hirsch method cannot be utilized while a modification to the original Bingel reaction without the use of any templating agent was needed for a highyield synthesis of the compound. Differently the Bingel-Hirsch reaction was used with $C_{60}$ and 2-methacryloyloxyethyl-Me-malonate or 2-methacryloyloxyethyl-dichloroacetate to produce fullerene-containing methacrylates [111].

Same reaction was used to produce a bis-malonate $C_{60}$ derivative with terminal alkyne groups [112]. The reaction was carried out using copper-catalyzed azide-alkyne cycloaddition to produce a series of new fullerene glycoconjugate derivatives. $\mathrm{C}_{60}$ and an enantiopure bismalonate tether equipped with two acetonide moieties were Bingel-reacted to produce an enantiomerically pure [60] Fullerene bisadducts [113]. The produce combines the inherent chirality of the fullerene core with the functional glycol groups located on the 
tether. A variation of the Bingel reaction based on cyclopropanation of [60] fullerene with bromo-substituted active methylene compounds was used to produce methanofullerene in the presence of amino acid and DMSO without the use of catalysts [114]. More information about the fullerene functionalization chemistry including the use of the Bingel reaction may be found in $[115,116]$

Among the different chemical processes available for functionalizing fullerenes, the Diels-Alder cycloaddition reactions are very versatile resulting in a large variety of cycloadducts (see Figure 5A). The fullerene being electron-deficient reacts with electron-rich 1,3-dienes to form adducts in which a 6-6 bond is fused to a cyclohexene ring. An example is the reaction of $C_{60}$ with anthracene or other 1,3-dienes [116]. Diels-Alder cycloaddition was also utilized for reacting 2-[(trimethylsilyl)oxy]buta-1,3-diene with $\mathrm{C}_{60}$ after hydrolysis and reduction, obtaining additional functional groups such as $\alpha$-amino acid derivatives could be attached through esterification. There is a large variety if cycloaddition reactions utilized to modify the fullerene surface chemistry upon desired properties [116-120] as shown in Figure 5B,C.

A variety of reactions can be performed to attach complex molecules to fullerenes. Some of the functionalization reactions are sketched in Figure 6.

\subsection{Carbon Onion Synthesis}

Concerning carbon nano-onions, besides electron irradiation there are several methods for the synthesis developed at the end of the 20th century. CNOs can be produced by decomposition of are acetylene, boron trichloride and ammonia precursors using hydrogen as a carrier via chemical vapor deposition (CVD) [121]. The resulting CNOs are containing quasi-stoichiometric boron-nitride domains. In another work CNOs were produced by decomposition of methane via CVD with the help of a Ni/Al catalyst. CVD was also utilized by other authors to synthesize metal-containing CNOs [122]. Firstly, a sol-gel containing $\mathrm{Ni}$ and $\mathrm{Sn}$ was spin-coated on a Si substrate and dried and annealed to $600{ }^{\circ} \mathrm{C}$ to obtain the catalysts. Then decomposition of a mixture of methane and hydrogen was performed by CVD leading to the formation of the CNOs containing $\mathrm{Ni}_{3} \mathrm{Sn}_{2}$ core. $\mathrm{Ni}$ was utilized as a catalyzer also in a counterflow diffusion flames to synthesize CNTs and CNOs [123]. A mixture of 5\% ethylene and $15 \%$ to $45 \%$ methane and the remaining part oxygen was supplied in an upper burner while a mixture of oxygen and nitrogen was used in a lower burner. In low flow rate conditions, a quasi-stable one dimensional flame with working temperatures in the range $400-1200{ }^{\circ} \mathrm{C}$ is obtained. Changing the ethylene, methane and oxygen concentrations, the decomposition of methane led to the formation of high yield of CNTs or CNOs. A combustion flame was also utilized burning naphthalene to produce gram-scale of CNOs [124]. Other precursors as phenolic resins [125] and methods to improve the efficiency of the CNOs production are make use of resonant excitation of precursor molecules [126], acoustic modulation of the laminar flame [127]. CNOs may also be obtained transforming nanodiamonds via thermal treatment in vacuum at $\sim 1700{ }^{\circ} \mathrm{C}$ [128]. The process starts at the surface with the formation of a graphitic outer shell on the diamond core which then transforms into a carbon onion surrounding an inner $\mathrm{sp}^{3}$ phase. Increasing the temperature, the diamond phase transforms in a facetted graphitic structure. In another study, the evolution of the diamond to onion transformation as a function of the temperature was analyzed [129].

Depending on the process, detonation nanodiamonds obtained at high temperatures (up to $2000{ }^{\circ} \mathrm{C}$ ) and high pressure (up to $200 \mathrm{GPa}$ ), were purified from soot in $\mathrm{H}_{2} \mathrm{SO}_{4}$ and $\mathrm{HClO}_{4}$ acids and then heated in vacuum at temperature ranging from $900{ }^{\circ} \mathrm{C}$ to $1400{ }^{\circ} \mathrm{C}$. The analysis of the evolution of the diamond to onion transformation, shows that the graphitization process starts at $900{ }^{\circ} \mathrm{C}$ and proceeds with increasing the temperature till a complete transformation of the diamond phase into onions at $1400{ }^{\circ} \mathrm{C}$. The type of precursor and the synthesis conditions have a strong impact on the structure, as seen from transmission electron micrographs of different types of carbon onions given in Figure 7 , but all carbon onions share the multi-shell fullerene-like architecture. 
(A)
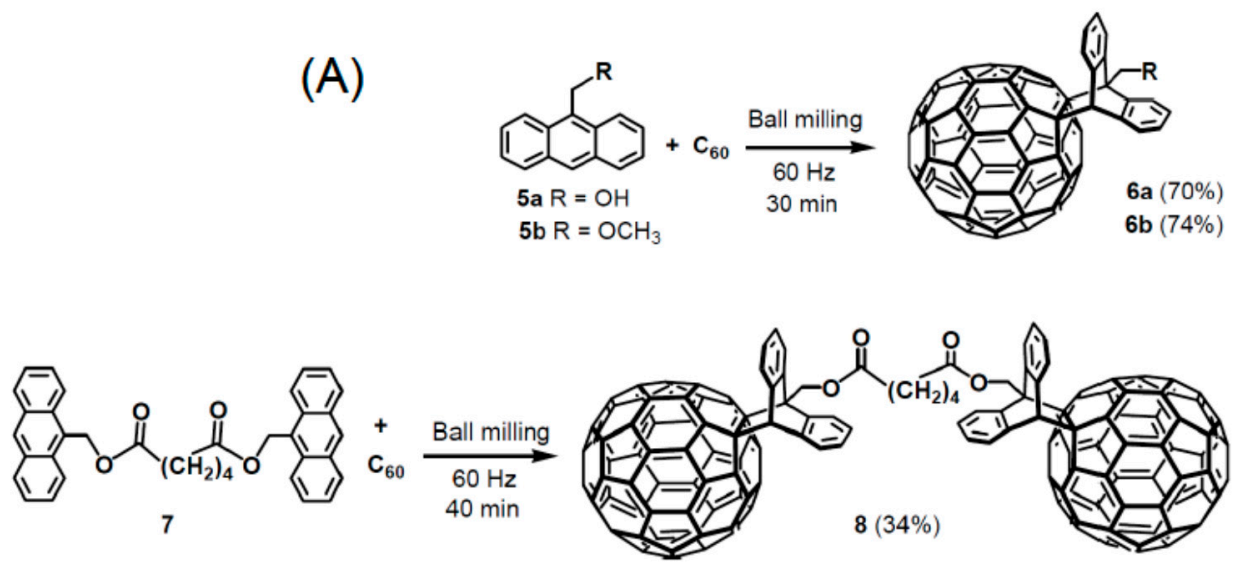

(B)

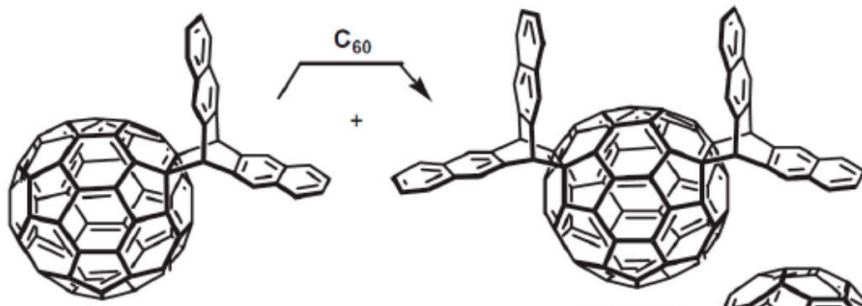

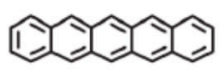

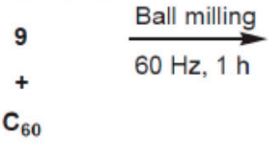

$10(27 \%)$
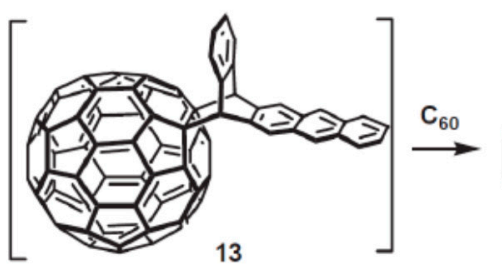

$11(21 \%)$
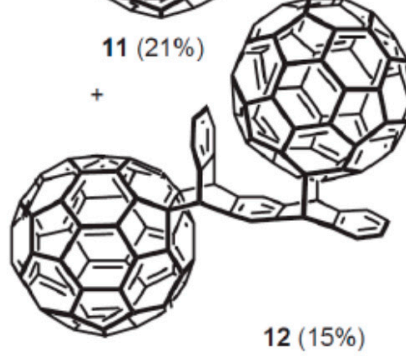

(C)

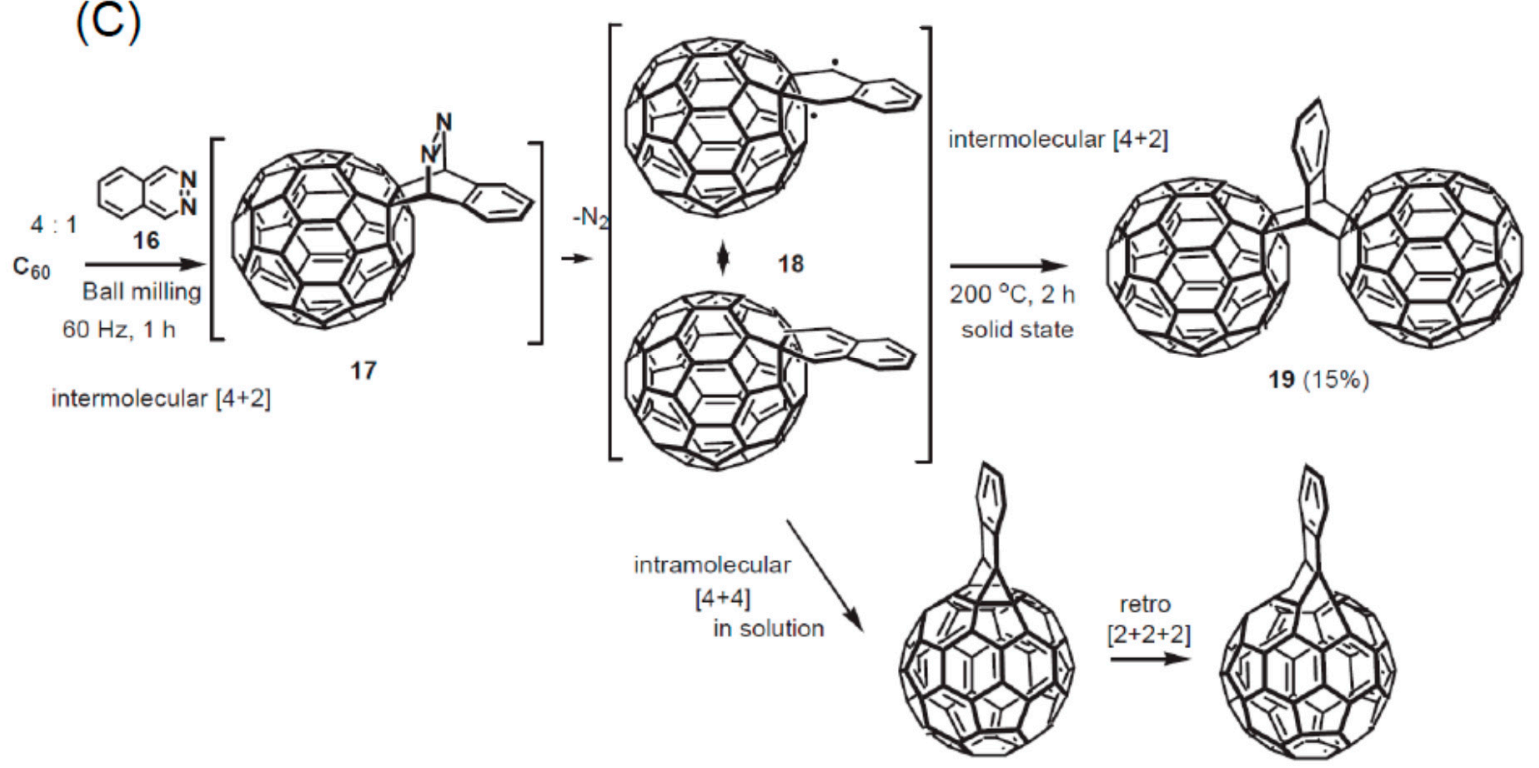

Figure 5. (A) Diels-Alder reactions of fullerene with 9-substituted anthracenes, (B) reaction of fullerene and pentacene, (C) reaction of fullerene and phthalazine. Reprinted with permission from [120]. Copyright 2016 Elsevier. 
(A)

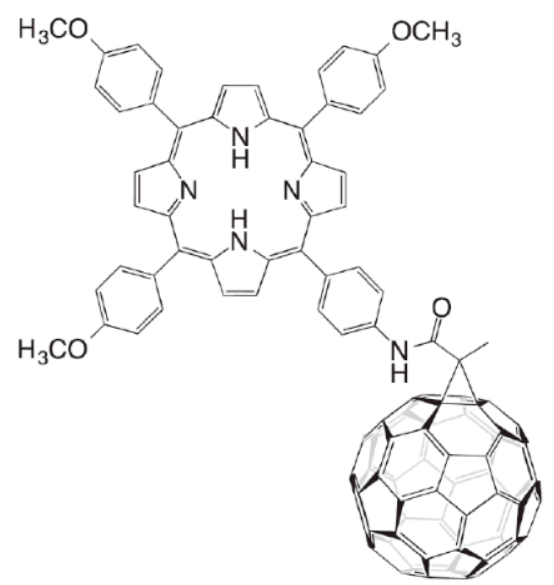

(B)

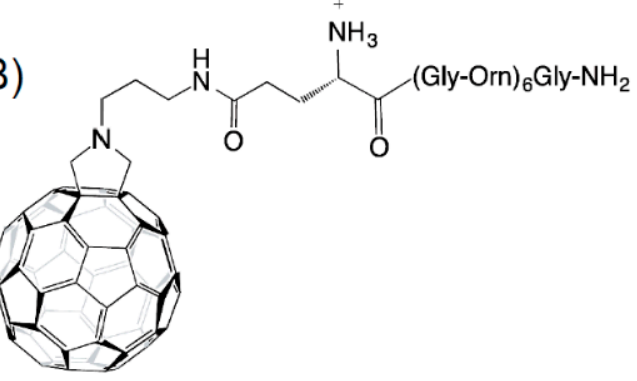

(C)
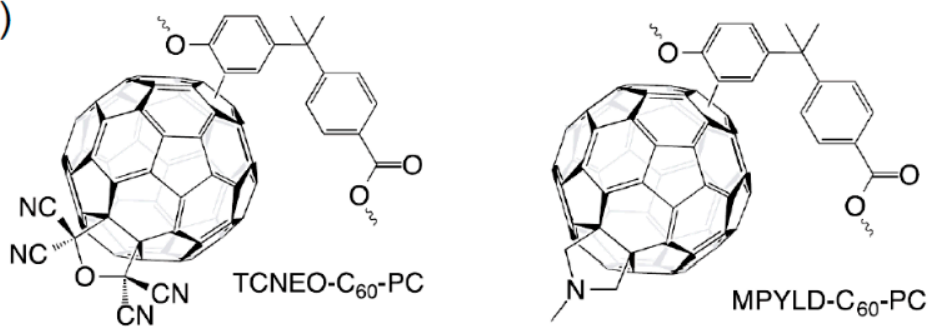

Figure 6. (A) Molecular structure of PS porphyrin-C60 dyad; (B) fullerene peptide fabricated via solid-phase peptide synthesis from amino functionalized fullerene and N-Fmoc-L-glutamic acid a-tert-butyl ester. (C) Polycarbonate containing fullerene derivatives. Reprinted with permission from [120]. Copyright 2017 Elsevier.
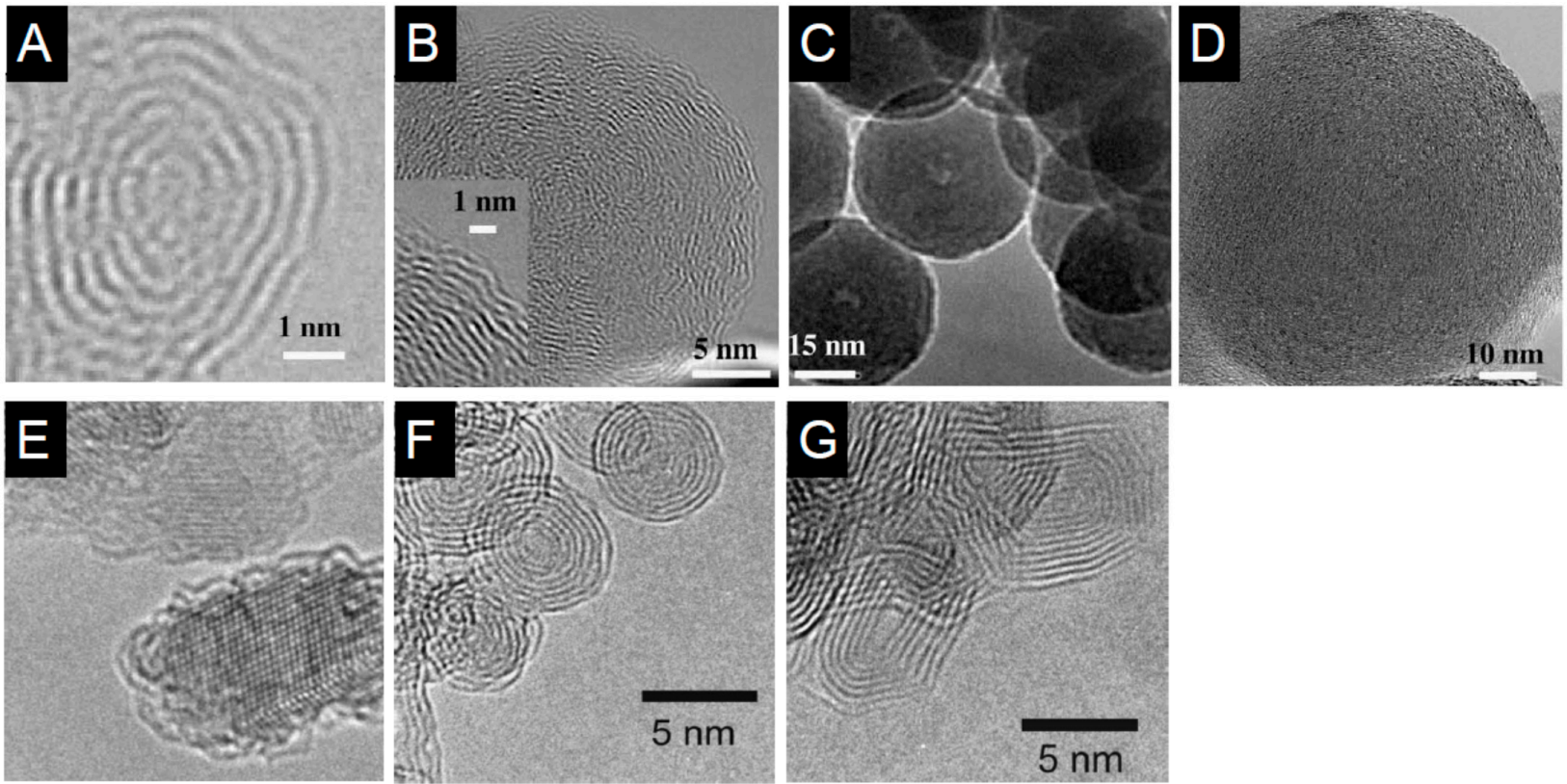

Figure 7. HRTEM images of CNOs produced by a counter-flow diffusion flame process as a function of the methane precursor concentration: (A) $\mathrm{CH}_{4}=15 \%$, (B) $\mathrm{CH}_{4}=25 \%$, (C) $\mathrm{CH}_{4}=35 \%$, and (D) $\mathrm{CH}_{4}=45 \%$ (adapted with permission from [123]. Copyright 2009 Elsevier.). Nano-diamond to CNOs transformation as a function of the temperature. HRTEM images of (E) diamond nanoparticles starting material, (F) spherical carbon onions, and (G) polyhedral carbon onions: Diamond nanoparticles are transformed into spherical onions at about $1700{ }^{\circ} \mathrm{C}$. Polyhedral onions are dominant in the sample annealed above $1900{ }^{\circ} \mathrm{C}$ (adapted with permission from [130]. Copyright 2001 AIP Publishing). 


\subsection{Carbon Nano-Onion Functionalization}

Similarly to fullerenes also pristine CNOs show different solubility in polar, nonpolar, protic and aprotic solvents [131]. The different behavior respect to solvents can be explained through their capability to be good proton acceptors as in the case of N-Ndimethylformamide. This leads to negative $\zeta$-potentials values and good CNOs solubility. Differently, as in the case of chloroform, tetrachloroethane, CNOs are unable to donate protons to the medium. This leads to a positive $\zeta$-potential thus resulting in a poor solubility. Increasing the solubility may be obtained functionalizing the $\mathrm{CNO}$ surface. The presence of curvature renders the $\mathrm{CNO}$ functionalization rather easy via covalent and non-covalent reactions. One of the common reactions is the oxidation of $\mathrm{CNO}$ s obtained using oxidative or reductive reagents such as $\mathrm{HNO}_{3}$ or $\mathrm{H}_{2} \mathrm{SO}_{4}$ or $\mathrm{KOH}$. However, the use of strong acids or bases can damage the CNOs. These strong acids introduce ether-like, hydroxyl, carboxyl functional groups. An alternative route is the use of nitric acid or ozone to attack the CNOs surface grafting oxygen functional groups [132]. Carboxyl groups and azomethine ylide addition (1,3-dipolar cycloaddition) reactions were also utilized to make CNOs soluble [133]. Further reactions with carboxylated CNOs may involve direct acid-base interaction, amidation via in situ generated acid chloride, and carbodiimide-activated coupling. For example, amidation of carboxylated CNOs is performed by direct reaction with diamine terminated polyethyleneglycole. Another possible amidation reaction is obtained reacting carboxylated CNOs with 1-Octadecylamine described as a very good agent for the amidation for CNTs [134]. Introduction of NH groups may be obtained also by microwave irradiation of CNOs suspended in dimethylformamide [133]. Finally, 1,3-dipolar cycloaddition of azomethine ylide is another route to functionalize the CNOs surface [133,135]. The azomethine ylides can be produced by condensation of an $\alpha$-amino acid and an aldehyde, a reaction which was extensively applied to the organic modification of fullerene $C_{60}$ [101]. In another example, a [2+1] Bingel-Hirsch cyclopropanation was used to functionalize the CNOs in presence of dodecyl malonate ester, carbon tetrabromide and 1,8-diazabicyclo[5.4.0]undec-7-ene [136].

Well-dispersible CNOs can be obtained through nucleophilic substitution [137]. The functionalization proceeds in two steps: first the CNOs are reduces in presence of Na-K alloy in 1,2-DME under vacuum, follows the covalent functionalization by an electrophile precursor (1-bromohexadecane). The alkylated CNOs exhibited high dispersion in polar solvents. A covalent $\mathrm{CNO}$ amidation can be performed using 4-aminopyridine, 1-octadecylamine (ODA), polyethylene glycol (PEG1500N), disulfide derivatives, [133,138,139]. Complexation of CNOs was also produced using the procedure indicated by Prato resulting in N-Boc-protected amino-CNOpyrrolidine [140]. Another interesting chemical modification of the $\mathrm{CNO}$ surface is the formation of $\mathrm{CNO}$ derivatives using folic acids polyethylene glycol and fluorescein species using a copper-catalysed azide-alkyne Huisgen cycloaddition [141]. Functionalized CNOs are used as both imaging and targeting cancer cells. The modified CNOs display high brightness and photostability in aqueous solutions, rapid uptake in two different tumor cell lines and negligible cytotoxicity. CNOs may be functionalized also attaching polymeric molecules. As an example using polypyrrole CNO the specific capacitance of supercapacitors are improved [142]. CNOs are coated with polypyrrole using chemical polymerization or electrostatic potentiostatic deposition to form bilayers. The composites obtained with the two techniques show higher mechanical and electrochemical stabilities, with high specific capacitances with specific capacitances of $800 \mathrm{Fg}^{-1}$ and $1300 \mathrm{Fg}^{-1}$ respectively. Other non-covalent functionalization of the $\mathrm{CNO}$ surface include surfactants as and poly(4-vinylpyridine-co-styrene) (PVPS) or poly(ethylene glycol)/polysorbate 20 (PEG-P20). Successive attachment with 3-mercaptopropionic or 2-mercapto-4-methyl-5-thiazoleacetic acids enabling binding phenolic compounds as quercitine [143]. Another example is the modification of CNOs with PEG to attach phenolic compounds as 1,2,3,4,6-penta- $\beta$-O-galloyl-d-glucopyranose ( $\beta$ PGG) and gallic acid molecules known for their protecting properties on mammalian cells. The CNO/PEG systems may be used as signal amplifier in the analytical biosensors or 
for drug targeting applications. Complete description of the surface functionalization of CNOs may be found in $[133,144]$.

\subsection{Fullerenes Sensing}

Technology and automation are even more expanding in our everyday life and this requires an even more distributed use of sensors. Some of the potential applications of sensors are in biomedical industry, electronics and automotive industries, environmental monitoring, agricultural and food industries, defense and homeland security. In all these applications the integration of sensors into intelligent devices and systems require improved sensitivity, selectivity and stability to measure, analyze physical entities. Carbon nanostructures including fullerenes, are materials of choice due to their superior properties and we will report here some applicative examples.

Fullerene films and fullerene compounds with iodine are used to fabricate temperature and pressure sensors [145]. Fullerene films of 2-3 $\mu \mathrm{m}$ thickness were deposited by evaporation at a temperature of $600-680^{\circ} \mathrm{C}$. Authors demonstrated that the film resistivity is sensitive to the temperature the humidity and the pressure [145]. Sensitivity of the sensor increases if the surface of the fullerenes films is oxidized. Another moisture sensor was fabricated immobilizing the $\mathrm{C}_{60}$ nanoparticles on an alumina substrate [146]. Fullerenes led to a corrugated surface with increased specific surface, resulting in an enhancement of the device sensitivity enabling moisture detection at the ppm level. Thanks to the small pore size of the active surface, this device has the potential to be used successfully in the gas, oil, and food industries.

In electrochemical sensors, electrodes are used as transducers of redox chemical reactions. Among the materials carbon derivative electrodes can be used in combination with the immobilized recognition agent for obtaining high analyte selectivity. Electrochemical sensors may be classified in amperometric if an electroactive species is formed at the electrode, in potentiometric it the electrode detects presence of ions or impedimetric if the coupling with an external analyte leads to a change of the electrode impedance $[147,148]$. It is well known the electron accepting ability of $\mathrm{C}_{60}$ molecules. This property has been exploited to modify many substrates to lower their potential of electroreduction and increasing the reaction rates, improving sensitivity and selectivity in electrochemical sensors [149]. For these properties, fullerenes have been used both in mediators electrochemical catalysis and as redox catalysts $[149,150]$. Electrodes modified with fullerenes can be used biomolecular sensing and environmental monitoring [151,152]. As an example, nanoporous fullerene $\mathrm{C}_{60}$ crystals were obtained via self-assembling process. The $\mathrm{C}_{60}$ crystal showed an excellent sensing property in particular for aromatic vapors, due to the easy diffusion through the porous crystal architecture and strong $\pi-\pi$ interaction between the aromatic rings [153]. Fullerenes were also utilized to detect toxic substances as bisphenol. The sensor showed linear behavior in a concentration range $74 \mathrm{nM}-0.23 \mu \mathrm{M}$ [154]. In another work, authors utilized fullerenes derivatives and artificial macrocyclic polyethers to coat a quartz crystal [155]. The oscillating frequency of the quartz crystal changed as a result of the adsorption of organic or inorganic molecules on coating material. The sensor exhibited a short response time $(<2.0 \mathrm{~min}$.) and a high sensitivity, good selectivity, and good reproducibility for polar organic gases.

$\mathrm{C}_{60}$ was also used to drop-coating a film onto an electrode surface which was next coated with a protective Nafion film [156]. Authors showed a quasi-reversible one-electron electroreductions at this fullerene modified electrode CME. Oxygen dissolved in a $2 \%$ $\left(\mathrm{C}_{2} \mathrm{H}_{5}\right)_{4} \mathrm{NOH}$ water-DMF $(3: 2, v: v)$ solution was electrocatalytically reduced at his electrode and the more negative was the redox potential $\mathrm{E}^{\circ}$ value, the more reversible was the electrochemical process. Therefore, this electrode may find practical application as a sensor. Alternative highly sensitive oxygen sensors rely on optical properties of $C_{70}$ [157-159]. In the last work, authors utilized isotopically enriched carbon-13 fullerene $C_{70}$ dissolved in polymer matrixes characterized by different oxygen permeability, as polystyrene (PS), ethyl cellulose (EC) and an organically modified silica gel (OS). Rapid lifetime determination 
method was applied to determine oxygen concentration. At room temperature, the $\mathrm{C}_{70}$ based sensor had a very low LOD dependent on the polymer used: $250 \mathrm{ppbv}$ for EC, 320 ppbv for OS and $\sim 530 \mathrm{ppbv}$ for PS. In another work, fullerene nanorods were synthesized by liquid-liquid interface and immobilized on the surface of a glassy carbon electrode [160]. To produce a highly conductive electrochemically reduced fullerene nanorod the electrode was electrochemically reduced in $1.0 \mathrm{M}$ potassium hydroxide $(\mathrm{KOH})$. The electrode was utilized for sensing ethylparaben (EP) in a concentration range from $0.01-0.52 \mu \mathrm{M}$ and showed a LOD $3.8 \mathrm{nM}$. A stable non-enzymic electrochemical sensors was fabricated coupling zinc porphyrin and fullerene $\left(\mathrm{ZnPp}-\mathrm{C}_{60}\right)$ [161]. Then $\mathrm{ZnPp}-\mathrm{C}_{60}$ was entrapped in tetraoctylammonium bromide film on glassy carbon electrode. The electrode showed excellent reproducibility with extremely fast response in sensing $\mathrm{H}_{2} \mathrm{O}_{2}$ in the range 0.035 to $3.40 \mathrm{mM}$, with LOD of $\sim 0.81 \mu \mathrm{M}$.

Thanks to the biocompatibility of carbon nanomaterials, a wide area of fullerene application is the biosensing where they show sensitive interaction with analytes, efficient transduction of the biorecognition events, and fast response times. The biosensor is based on a transducer element able to convert an interaction with biological molecules into an electrical signal. Among the interactions, that with DNA is very important in life science being in direct contact with the transcription processes, mutation of genes, origins of diseases, and molecular recognition studies [162]. Biorecognition of DNA was accomplished by using $\left[\mathrm{Co}(\mathrm{phen})_{3}\right]^{3+/ 2+}$ as an appropriate electroactive element [163]. In particular, the interaction with DNA led to a decreased peak current and the electrode recovered significantly in the presence of $\mathrm{H}_{10} \mathrm{C}_{60}\left(\mathrm{NHCH}_{2} \mathrm{CH}_{2} \mathrm{OH}\right)_{10}$. An ultra-sensitive fullerene-modified biosensor was made to detect miRNA-141 [164] as depicted in Figure 8.

In the first step A, two sequences containing G-quadruplex were connected by click chemistry-mediated nucleic acid strands as shown in Figure 8. To separate the miRNA, the obtained complete G-quadruplex was used to form DNA-RNA hybrid duplexes. Subsequently, the DNA parts of the duplexes were cleaved and the miRNA-141 was released. The second step B consists in a signal amplification by enzyme-assisted target recycling. In this respect multi-labeled functionalized fullerene nanoparticles (FC60) thiol-attached to an $\mathrm{Au}$ electrode, provides a large surface area with active sites to obtain multiply-enhanced amplified signal. The device sensitivity allowed detection of miRNA-141in the range $0.1 \mathrm{pM}$ and $100 \mathrm{nM}$, and the lowest LOD of $7.78 \mathrm{fM}$. Fullerenes were also utilized in the ink of screen printed electrodes to detect rDNA of Escherichia Coli [165]. The electrode was treated in a plasma to graft a DNA probe allowing a direct detection of the 16S rDNA extracted from Escherichia Coli with reduction of the redox peak of $\left[\mathrm{Co}(\mathrm{phen})_{3}\right]^{3+/ 2+}$ occurring only when perfect hybridization between $16 \mathrm{~S}$ rDNA sequence and probe strands occurred. An impedimetric Fetuin-A biosensor was fabricated by modifying a gold electrode activated EDC/NHS poly-hydroxylated fullerene and then coated with PAMAM (G5) [166]. Then anti-Fetuin-A antibodies were attached to the electrode surface enabling detection of Fetuin concentrations in the range between 5 and $400 \mathrm{ng} / \mathrm{mL}$ and the lowest LOD of $1.44 \mathrm{ng} / \mathrm{mL}$. Compared to ELISA test the sensor provides linear behavior and fast response time. Another ultrasensitive electrochemical sensor based on fullerenes was fabricated to detect Mycobacterium tuberculosis (MTB) [167]. The sensor provided a rapid and efficient detection method for MTB with excellent specificity and sensitivity for MTB antigen in serum samples obtained from patients infected by tuberculosis. Authors produced fullerene $\mathrm{C}_{60}$-polyaniline $\left(\mathrm{C}_{60}-\mathrm{PAn}\right)$ nanohybrids possessing large surface area, high concentration of active groups and excellent electric performance. The nanoprobes were attached to gold nanoparticles and labeled with signal antigen MPT64 to form the tracer label. The electrochemical response signal is generated by the $\mathrm{C}_{60}$-Pan-MPT64 and is further amplified by the electrocatalysis of ascorbic acid through $\mathrm{C}_{60}-\mathrm{Pan}$. The device showed a linear detection in the range from 0.02 to $1000 \mathrm{pg} / \mathrm{mL}$ with a LOD $20 \mathrm{fg} / \mathrm{mL}$ for MPT64. More importantly, it also exhibited excellent specificity and sensitivity for MPT64 detection in serum samples of tuberculosis patients, which provided a rapid and efficient detection method for MTB infection. 


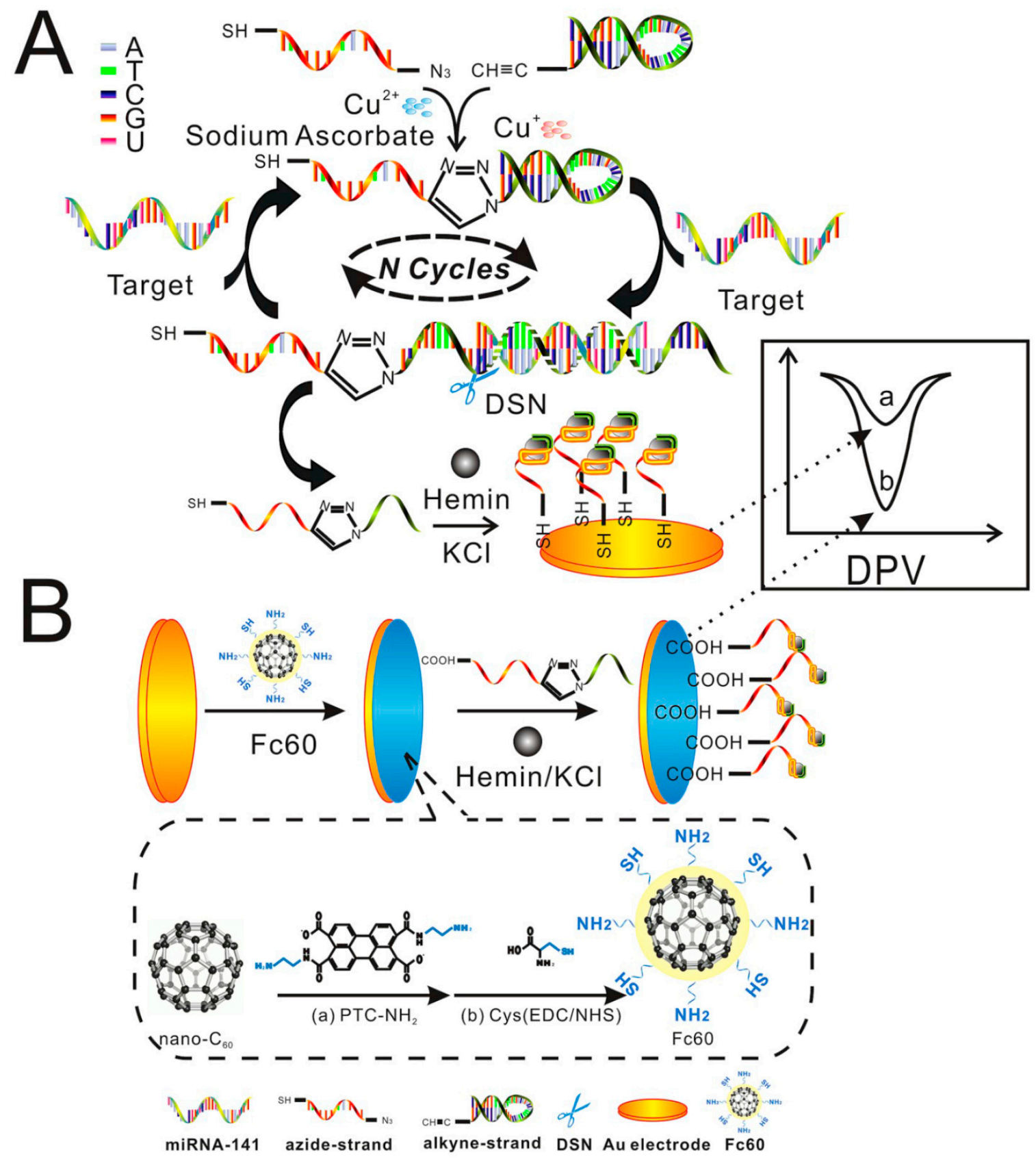

Figure 8. The schematic diagram of the EATR (A) and FC60 dual-amplified strategy electrochemical biosensor (B) (reproduced with permission from [164]. Copyright 2020 Elsevier.).

Fullerenes are also used in glucose sensors. In [168] glucose oxidase (GOD) electrochemistry observed using a glassy carbon electrode modified with GOD-hydroxyl fullerenes. In another work a glucose sensor was produced using a mixed-valence cluster of cobalt(II) hexacyanoferrate and fullerene $\mathrm{C}_{60}-\mathrm{GOD}$ enzyme [169]. The $\mathrm{C}_{60}$-GOD enzymebased glucose sensor showed linear response up to $8 \mathrm{mM}$ glucose with a sensitivity of $5.60 \times 10^{2} \mathrm{nA} / \mathrm{mM}$ and a $5 \mathrm{~s}$ fast response time. Finally, the sensor also showed a detection limit of $1.6 \times 10^{-6} \mathrm{M}$ and a high reproducibility. Glucose was also detected using a piezoelectric quartz crystal [170]. Authors used GOD functionalized fullerenes to catalyze the oxidation of glucose. The production of gluconic acid was then detected by a piezoelectric quartz crystal sensor obtaining a good lower limit of detection of $3.9 \times 10^{-5} \mathrm{M}$ for glucose in aqueous solutions. Fullerenes were also used to fabricate urea sensors [171]. The urease enzyme was immobilized on $\mathrm{C}_{60}$ and subsequently deposited on a screen-printed electrode 
containing a non-plasticized poly(n-butyl acrylate) membrane entrapped with a hydrogen ionophore. In optimal $\mathrm{pH}$ condition, the biosensor led to a linear response in the range from $2.31 \times 10^{-3} \mathrm{M}$ to $8.28 \times 10^{-5} \mathrm{M}$. Presence of cations such as $\mathrm{Na}^{+}, \mathrm{K}^{+}, \mathrm{Ca}^{2+}, \mathrm{Mg}^{2+}$ and $\mathrm{NH}_{4}{ }^{+}$did not influence the response of the urea biosensor. Sensing modalities analytes and sensor performances are summarized in Table 1.

Table 1. Detection modality, analyte and detection limit of fullerene-based sensors.

\begin{tabular}{|c|c|c|c|c|}
\hline Method & Functionalization & Analyte & LOD & Reference \\
\hline Resistivity & $\mathrm{C}_{60}$ films & $\begin{array}{l}\text { Temperature, } \\
\text { Pressure }\end{array}$ & $2 \% /{ }^{\circ} \mathrm{C}$ & [145] \\
\hline Chronoamperometry & Pd@Cys- $\mathrm{C}_{60}$ & Glucose & $1 \mu \mathrm{M}$ & [172] \\
\hline Amperometric & Carboxylated $\mathrm{C}_{60}$ & Glucose & $2 \mathrm{mg} / \mathrm{mL}$ & [149] \\
\hline Amperometric & $\mathrm{C}_{60}-\mathrm{GOD}$ & Glucose & $1.6 \times 10^{-6} \mathrm{M}$ & [169] \\
\hline Piezoelectric & $\mathrm{C}_{60}-\mathrm{GOD}$ & Glucose & $3.9 \times 10^{-5} \mathrm{M}$ & [170] \\
\hline Potentiometric & $\mathrm{C}_{60}$-urease & Urea & $8.28 \times 10^{-5} \mathrm{M}$ & [171] \\
\hline Microbalance & $\mathrm{C}_{60} \mathrm{SAM}\left({ }^{1}\right)$ & $\begin{array}{l}\text { VOCs }\left({ }^{2}\right) \text { of methanol, hexane, } \\
\text { benzene, toluene, aniline }\end{array}$ & High gas selectivity & [153] \\
\hline Capacitive & $\mathrm{C}_{60}$ Alumina & Moisture & $1 \mathrm{ppm}$ & [146] \\
\hline Voltammetry & $\mathrm{C}_{60}$ nanorods & Ethylparaben & $3.8 \mathrm{nM}$ & [160] \\
\hline Amperometric & $\mathrm{ZnPp}-\mathrm{C}_{60}$ & $\mathrm{H}_{2} \mathrm{O}_{2}$ & $\sim 0.81 \mu \mathrm{M}$ & {$[161]$} \\
\hline Cyclic voltammetry & NiNP-CuNP-C 60 & Vitamin $\mathrm{D}_{3}$ & $0.00241 \mu \mathrm{M}$ & {$[173]$} \\
\hline Amperometric & Cys-PTC-NH $2-\mathrm{C}_{60}$ & miRNA-141 & $7.78 \mathrm{fM}$ & [164] \\
\hline Impedimetric & $\begin{array}{l}\text { poly-hydroxylated } \\
\text { fullerene }\end{array}$ & Fetuin-A & $1.44 \mathrm{ng} / \mathrm{mL}$ & [166] \\
\hline Amperometric & $\mathrm{C}_{60}-\mathrm{PAn}$ & Mycobacterium tuberculosis & $20 \mathrm{fg} / \mathrm{mL}$ & [167] \\
\hline $\begin{array}{l}\text { Differential pulse } \\
\text { Voltammetry }\end{array}$ & $\mathrm{ACV}-\mathrm{C}_{60}$ & Acylovir & $1.48 \mathrm{nM}$ & [174] \\
\hline $\begin{array}{l}\text { Electrochemical } \\
\text { impedance } \\
\text { spectroscopy }\end{array}$ & $\mathrm{C}_{60}$-Polyacrilamide & Cortisol & $0.14 \mathrm{nM}$ & [175] \\
\hline Capacitive & $\begin{array}{c}\mathrm{C}_{60} \text {-Amide } \\
\mathrm{C}_{60} \text {-carboxyl } \\
\mathrm{C}_{60} \text {-uracil }\end{array}$ & ATP & $0.31 \mathrm{mM}$ & [176] \\
\hline Piezoelectric & Anti-IgG-PVA-C 60 & $\operatorname{IgG}$ & $0.1 \mu \mathrm{g} / \mathrm{mL}$ & [177] \\
\hline
\end{tabular}

$\left({ }^{1}\right)$ : SAM: self assembled monolayer. $\left({ }^{2}\right)$ VOCs: Volatile Organic Compounds.

\subsection{Carbon Nano-Onion Sensing}

As for CNOs, it has been demonstrated that polarization of the electronic states of fullerenes are preserved in CNOs. However, with respect to free fullerenes, the absorption energies of CNO states are significantly red-shifted allowing their use as photosensitizers in nanotechnology applications [178]. Luminescent properties of CNOs are also proposed in [179] as fluorophores. CNOs modified using a coupling the multicomponent Ugi reaction with a complex Pd-mediated cascade to attach a deep blue emitting furo[2,3-c]isoquinolines to $\mathrm{CNO}$ surface which renders them soluble.

Since CNO's physical and chemical properties resemble those of fullerenes, also the sensing applications are similar. CNOs were used to produce a hydrogen sensor thanks to their hydrophobic nature and their non-porous texture [180]. The $\mathrm{CNO} / \mathrm{C}_{2} \mathrm{H}_{6} \mathrm{O}$ sensor displayed a fast decrease in resistance with $\mathrm{H}_{2}$ concentration under 10 ppm making CNOs a good active element for the detection this gas. The decreased resistance can be attributed to the change of the CNOs electrical properties in a n-type semiconductor with an increase of 
the number of electrons in the conduction band. The electrical behavior of CNOs is similar to that found by other authors [181]. They tested the conductivity of CNOs in presence of different gaseous species as nitrogen, oxygen, hydrogen and methane. The gasses acted as electron donors for CNO semi-metallic electronic structure of CNOs leading to a decrease of their conductivity. In another work, palladium nanoparticles were deposited on onion-like mesoporous carbon vesicle to sense hydrazine [182]. Pd nanoparticles act as a catalyzer facilitating the hydrazine oxidation at a more negative potential and delivers higher oxidation current with respect the simple onion-like structure. The sensor provided a linear behavior from $2.0 \times 10^{-8}$ to $7.1 \times 10^{-5} \mathrm{M}$ and a low LOD of $14.9 \mathrm{nM}$ for hydrazine. Same authors developed also an amperometric sensor for hydrogen peroxide [182]. A glassy carbon electrode modified with a mesoporous onion-like coating decorated with $\mathrm{Pd}$ nanoparticles was developed. The electrode showed a linear enhanced amperometric responses towards hydrogen peroxide in the range from $1.0 \times 10^{-7}$ to $6.1 \times 10^{-3} \mathrm{M}$. In addition, the electrode showed a fast response of $1 \mathrm{~s}$ achieving the $95 \%$ of the steady-current in presence of hydrogen peroxide. Phosphorus doped CNOs are utilized to fabricate highly sensitive devices for $\mathrm{NH}_{3}$ [183]. The sensing mechanism is based on chemisorbed oxygen on the P-doped CNOs resulting in a charge transfer from $\mathrm{C}$ atoms to oxygen atoms. These last are adsorption site for $\mathrm{NH}_{3}$ molecules which reacting with oxygen atoms form $\mathrm{H}_{2} \mathrm{O}$ molecules and let the CNO surface less conductive. Then a measure of the device resistance can be correlated with the presence of ammonia gas in a range $10 \mathrm{ppb}-1 \mathrm{ppm}$ with a LOD of $10 \mathrm{ppb}$.

Another interesting application of CNOs is the electrochemical sensing of the $\mathrm{pH}$ [184]. A glassy carbon electrode was modified with a CNO deposited by electropolymerization. This modification induced a high chemical and electrochemical stability over a of 2-10 pH range with negligible interference of monovalent cations. The reproducibility and the lowcost easy fabrication open interesting perspectives in for the fabrication of miniaturized $\mathrm{pH}$ sensor devices. Some of the sensing modalities based on CNOs are summarized in Figure 9. The low toxicity of CNOs and their stability and high conductivity permit their use as sensors of biomolecules as hormones or enzymes. CNOs show high absorption in the UV region while its emission properties are wavelength dependent $[185,186]$. CNOs can be utilized directly as bio-imaging agent in living microorganisms [186].

There are different possible mechanisms explaining the CNO PL: the electron hole radiative recombination; the quantum confinement effects; the presence of emissive surface traps; dipole emitted centers; the electron-phonons coupling occurring in defects of CNO surfaces $[185,187]$. However, the need to detect specific molecules requires selectivity which is obtained through CNO surface functionalization [185] which renders the interaction between the analyte and the CNOs univocal. Functionalization was utilized to detect glucose using a "turn-off/turn-on" mechanism [185]. Methylene blue (MB) was adsorbed on the CNO surface via charge transfer and hydrophilic interactions. The charge transfer from CNOs to MB molecules caused the fluorescence to be turned off. CNO emission was recovered by the addition of glucose $\left(0.1 \mathrm{~mL}, 1.8 \times 10^{-2} \mathrm{M}\right)$. A possible mechanism is the H-bonding interaction between the nitrogen atom of the central ring of the $\mathrm{MB}$ molecules and the primary alcoholic group of glucose. This likely weakens the interaction between MB and CNOs leading to a "turn-on" of the fluorescence. The detection limit of this mechanism is $1.3 \times 10^{-2} \mathrm{M}$. Glucose was detected also using an amperometric sensing approach coupling CNOs to CNTs [188] or to Pt nanoparticles [189]. 
(A)

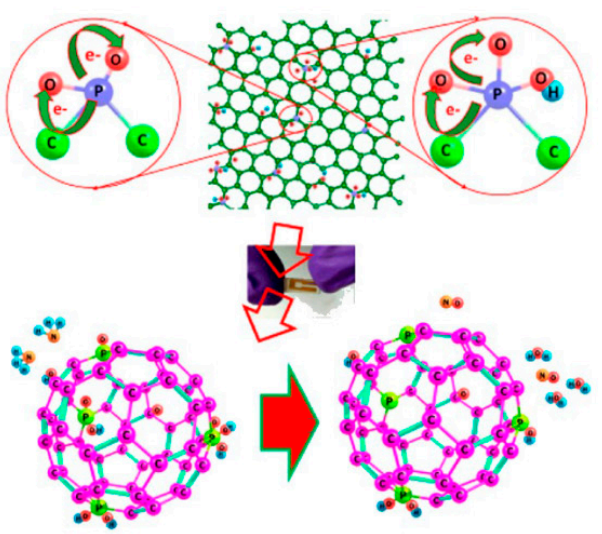

(C)

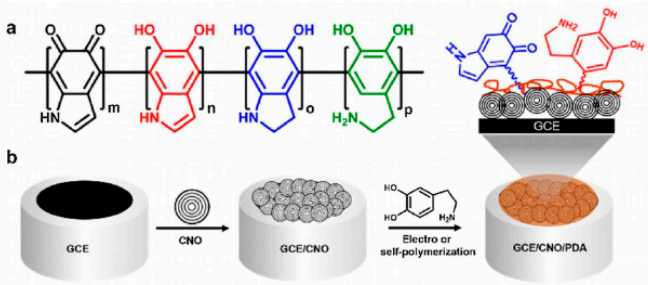

(B)

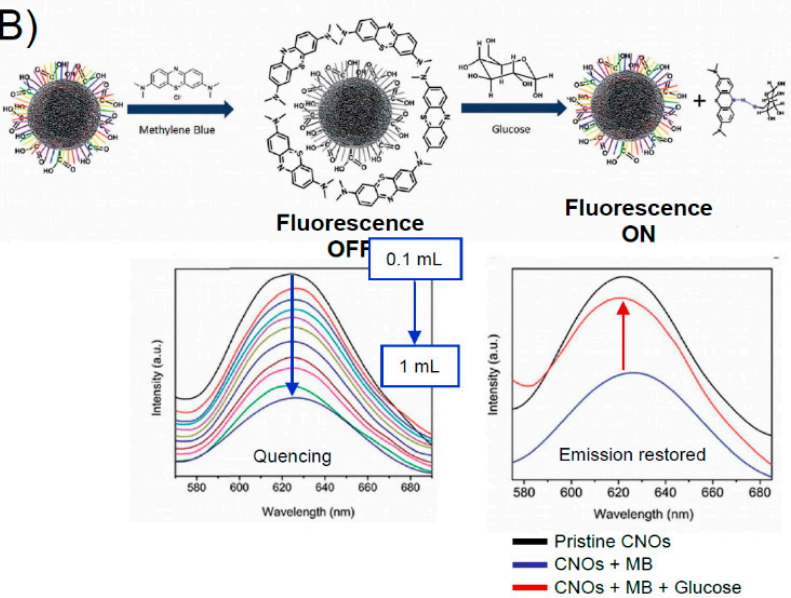

(D)

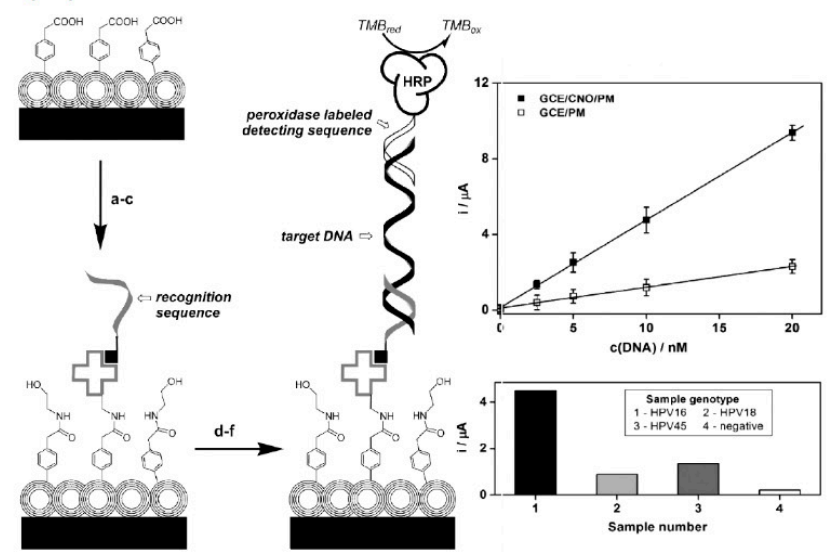

Figure 9. (A) electrochemical sensor based on CNOs: charge transfer between $\mathrm{CNOs}$ and $\mathrm{NH}_{3}$ molecules results in a change of the sensor resistance thus enabling detection of ammonia gas [183]. (B) Glucose sensor based on the optical properties of CNOs: addition of methylene blue quenches the natural emission from CNOs. Glucose molecules bind to MB thus reducing the interaction with CNOs and removing the quenching [185]. (C) Structure of polydopamine, and modification strategy of a glassy carbon electrode with polydopamine functionalized CNOs. Polydopamine voltammograms change as the $\mathrm{pH}$ changes thus enabling $\mathrm{pH}$ detection with potentiometric measurements [184]. (D) Fabrication of carcinoembryonic antigen-immunosensor based on a glassy carbon electrode modified with oxidized CNOs. Diazonoum salt was then used to functionalize the CNOs and sequentially immobilize streptavidin, biotinylated DNA, and target DNA. The hybridization of the target DNA with the DNA sequence of the papilloma virus can be detected by amperometric measurements [190]. Reprinted with permission from [183-185,190]. Copyright: 2019 American Chemical Society; 2018 Elsevier, 2016 RSC Publishing and 2009 Elsevier respectively.

The nanodiamond-derived CNOs sensing performances towards dopamine, epinephrine, and norepinephrine were studied in [191] revealing the high sensitivity, selectivity, and stability of the responses of the CNO-modified electrode. In particular authors found that in a concentration range of $0.1-6 \mu \mathrm{M}$ the oxidation peak current was proportional to the analyte concentration. For all the neurotransmitters the detection limit was found to be $100 \mathrm{nM}$. In other works CNOs were utilized for electrochemical sensing of dopamine in combination with carbon nanofibers (CNF) and polyacrylonitrile (PAN) [192]. The characterization of the materials indicates that the electron transfer properties decreases from CNOs $>$ CNOs-PAN $>$ CNOs-CNF $>$ PAN. The modified GCE were used as sensors for the dopamine using cyclic voltammetry (CV), square wave voltammetry (SWV), and electrochemical impedance spectroscopy. CNOs and CNOs-CNF gave comparable electrocatalytic activities in terms of sensitivity and LOD (CNOs $1.23 \mu \mathrm{M}$ and sensitivity of $0.74 \mu \mathrm{A} / \mu \mathrm{M}$, and CNOs-CNF $1.42 \mu \mathrm{M}, 0.31 \mu \mathrm{A} / \mu \mathrm{M}$ ). Surface of oxidized CNOs was modified using 
covalent interaction to link biotin-avidin molecules to fabricate an optical sensor [193]. The sensor was fabricated by layer-by-layer assembly utilized to modify a gold surface used to enable plasmon resonance. In addition, it was shown that also CNOs contribute to the amplification of the analytical signals of the biosensor. CNO based sensors find application also for the recognition of DNA strands. A DNA Sensors for Human Papillomavirus Oncogene Detection with Enhanced Sensitivity was fabricated modifying a glassy carbon electrode with CNOs [190]. These last are then functionalized with diazonium salts to bind streptavidin and then a recognition DNA sequence. This last is utilized to attach a target DNA sequence used to detect the papillomavirus oncogene via direct hybridization. This changes the electrochemical properties of the electrode thus allowing the hybridization to be detected through amperometric measurements. The sensor showed a higher sensitivity $(0.91 \mu \mathrm{A} / \mathrm{nM})$ and a lower limit of detection $(0.54 \mathrm{nM})$ with respect to the unmodified GCE (sensitivity $=0.21 \mu \mathrm{A} / \mathrm{nM}$ and a LOD $=3.9 \mathrm{nM}$ ). Table 2 summarizes characteristics of GCO sensors.

Table 2. Detection modality, analyte and detection limit of GCO based sensors.

\begin{tabular}{ccccc}
\hline Method & Functionalization & Analyte & LOD & Reference \\
\hline Potentiometric & PolyDopamine-OLC & $\mathrm{pH}$ & - & {$[184]$} \\
\hline Amperometric & Pd-OLC & Hydrazine, $\mathrm{H}_{2} \mathrm{O}_{2}$ & $14.9 \mathrm{nM}, 79 \mathrm{nM}$ & {$[182]$} \\
\hline Resistivity & $\mathrm{P}-\mathrm{OLC}$ & $\mathrm{NH}_{3}$ & $10 \mathrm{ppb}$ & - \\
\hline Potentiometric & PolyDopamine-OLC & $\mathrm{pH}$ & $<10 \mathrm{ppm}$ & {$[180]$} \\
\hline Resistivity & OLC/C ${ }_{2} \mathrm{H}_{6} \mathrm{O}$ & $\mathrm{H}_{2}$ & $1.3 \times 10^{-2} \mathrm{M}$ & {$[185]$} \\
\hline Fluorimetric & Oxidized OLC & Glucose & $1 \mathrm{mM}$ & {$[188]$} \\
\hline Amperometric & OLC-PDDA & Glucose & LOD $=3.9 \mathrm{nM}$ & {$[190]$} \\
\hline Amperometric & CNO-biotin & DNA & $100 \mathrm{nM}$ & {$[191]$} \\
\hline Cyclic voltammetry & CNO-PDDA & dopamine, epinephrine, and norepinephrine & dopamine & [192] \\
\hline Cyclic voltammetry & CNO & d &
\end{tabular}

\section{Nanodiamonds}

Diamond crystals are pure $\mathrm{sp}^{3}$ hybrids leading to a tetrahedral symmetry in which carbon atoms interact through strong covalent bonds. This perfect symmetric arrangement of the four orbitals of the valence electrons results in a structure with a density $\left(3.514 \mathrm{~g} \mathrm{~cm}^{3}\right)$ higher than that of graphite. This highly dense crystalline structure explains why diamond possesses an extraordinary strength, an unpaired resistance to compression, and hardness the highest of all other materials on both the Vickers and Mohs scales. The C-C bond properties also induce high chemical stability of diamond chemically also when in contact with strong acids. Diamond can react with the oxygen at a temperature of $\sim 700{ }^{\circ} \mathrm{C}$, leading to decomposition in $\mathrm{CO}, \mathrm{CO}_{2}$ [194]. Besides high resistivity, the diamond crystal is characterized by a very prominent phonon mobility corresponding to the highest heat conductivity of $3320 \mathrm{~W} / \mathrm{m}^{-1} \mathrm{~K}^{-1}$ at room temperature ( five times that of copper) [195].

Concerning electronic properties, the carbon-carbon strong covalent bonds causes diamond to be a wide-bandgap material of $\sim 5.5 \mathrm{eV}$ [196] characterized by a remarkable resistivity from $10^{11}$ to $10^{18} \Omega / \mathrm{m}$. Correspondingly, diamond possesses a high refracting index varying from 2.465 in the violet to 2.409 in the red [55]. This generates the prismatic colors of gemstones. The absorption of diamond is essentially due to the dopant exoatoms contained in the crystal. Diamond exhibits many different color centers. Nitrogen is the more common, boron, phosphorous, hydrogen, nickel, cobalt, silicon, germanium and sulphur are also frequently found in diamond crystals. Nitrogen defects are present in diamond crystals in a variety of forms called A-, B-, C- N2, N3 centers. In the visible, their characteristic absorption transitions fall at 575, 527, 478, 465, 452, 435, and $423 \mathrm{~nm}$ [197,198]. Diamonds are classified using the concentration of nitrogen defects. In $I a$ diamond the 
nitrogen impurities are $\sim 0.3 \%(3000 \mathrm{ppm})$ and includes about $95 \%$ of all natural diamonds. In type $I b$ diamonds the nitrogen impurities are up to $0.05 \%$ (500 ppm) and are about $0.1 \%$ of all natural diamonds. Type IIa diamonds are almost or entirely impurity free, colorless and constitute $\sim 1-2 \%$ of all natural diamonds. These diamonds possess the highest thermal conductivity. Finally, type $I I b$ diamonds have the lowest level of nitrogen impurities but contain significant boron impurities diamonds and amount to $\sim 0.1 \%$ of all natural diamonds.

\subsection{Nanodiamond Synthesis}

The diamond classification holds also for nanodiamonds which are obtained with top-down methods as the fragmentation of massive bulk diamonds. This means that the properties of fragmented NDs mirror those of their bulk counterpart in terms of biocompatibility, mechanical, optical, thermal, and electrical properties. Recently, the process of diamond fragmentation was studied to refine the production of high quality diamond powders [199]. Microcrystalline diamonds were compressed at different pressures (0.2-0.8 GPa) at ambient temperature to study the fragmentation process. The results show that with increasing pressure the fragmentation of the crystals proceeds through three stages: (i) fracturing of edges and corners, (ii) cracking of the crystal plane, and (iii) refinement of particle disorder. Experiments show also that increasing the pressure the particles reach a relatively stable size. Reduction of the size causes an increase of the contact area among the diamond particles with relaxation of the stress among the particles. As a consequence, the particle refinement vanishes. The production of macroscopic diamond crystals relies on two kinds of technologies, the HPHT and CVD which enable the production of single or polycrystalline diamonds at reasonable costs, fostering their use in diamond based technologies with outcomes in a variety of ordinary commercial products. CVD processes are utilized also to produce NDs $[200,201]$. In the first work NDs are obtained via dissociation of ethanol vapor in a microplasma at atmospheric pressure and neutral gas temperatures of $100{ }^{\circ} \mathrm{C}$. Addition of $\mathrm{H}$ in the plasma atmosphere, allows etching of the non-diamond phases and passivation of the ND surfaces. The size of the produced NDs is in the range 2-5 nm exhibiting cubic and lonsdaleite crystal structures. In the second work a CVD plasma process in a vertical configuration was utilized to synthesize high quality diamond crystals from nanometric to macroscopic dimensions. A laser induced plasma in liquid ethanol is also utilized to decompose the ethanol molecules and synthesize ND particulate [202]. The authors used a femtosecond laser with pulse repetition rate of $1 \mathrm{KHz}$ and a wavelength of $1030 \mathrm{~nm}$. The laser energy was tuned in the range 360-550 $\mu \mathrm{J}$ resulting in the formation of atomic $C$, ionized $C$ and $C_{2}$ clusters which acted as precursors for the formation of the nanodiamond crystals. Another used method for the production of NDs is the detonation process [203]. Three possible detonation processes are commonly utilized: (i) conversion of graphite or a carbon precursor into NDs in presence of catalysts at high pressure ( $7 \mathrm{GPa})$ and temperature (around $2000^{\circ} \mathrm{C}$ ) [204]; (ii) detonation of carbon precursors in a closed chamber using explosives. The process is based on shock waves leading to pressures in the range $20-100 \mathrm{GPa}$ and temperatures $>1700{ }^{\circ} \mathrm{C}$. Pressures and temperatures are high enough to induce a graphite to diamond conversion $[205,206]$; finally the third process relies on a mixture of $60 \mathrm{wt} \% \mathrm{TNT}\left(\mathrm{C}_{6} \mathrm{H}_{2}\left(\mathrm{NO}_{2}\right)_{3} \mathrm{CH}_{3}\right)$ and $40 \mathrm{wt} \%$ hexogen $\left(\mathrm{C}_{3} \mathrm{H}_{6} \mathrm{~N}_{6} \mathrm{O}_{6}\right)$ ) which are detonated in a closed metallic vessel in presence of $\mathrm{N}_{2}, \mathrm{CO}_{2}$ and $\mathrm{H}_{2} \mathrm{O}$ [207]. During the explosion the pressure rises to $\sim 28 \mathrm{GPa}$ and the temperature rises around $3800 \mathrm{~K}$ (Jouguet point) corresponding to liquid carbon clusters region. The decrease of the temperature leads to carbon cluster solidification in diamond nanocrystals. These processes lead to the formation of very small nanodiamonds with dimensions in the $1-5 \mathrm{~nm}$ and likely surrounded by a graphitic/amorphous carbon shell which is removed using strong acids [208]. Figure 10 shows the nanodiamonds produced by different methods. 

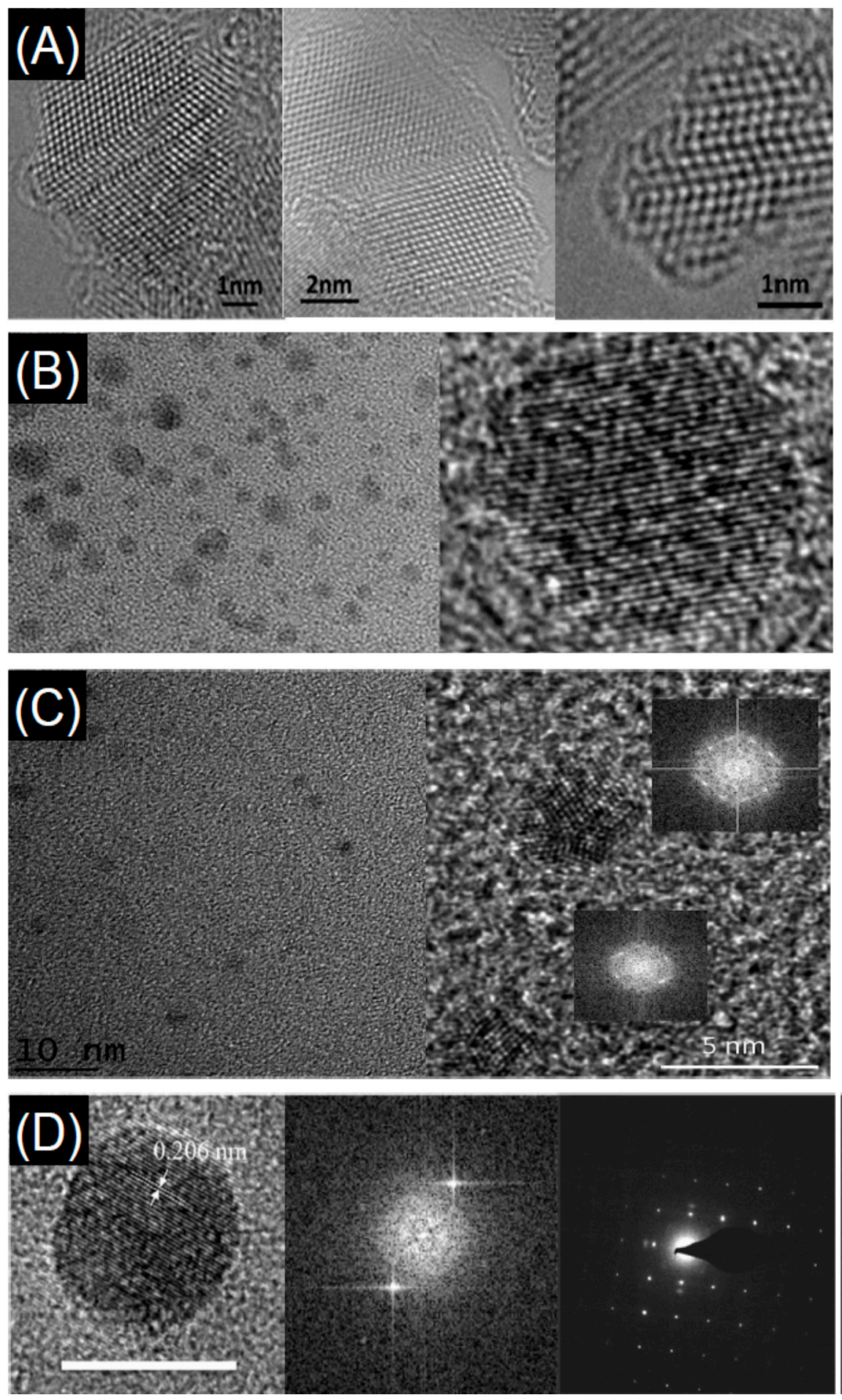

Figure 10. NDs synthesized (A) by detonation; (B) by pulsed laser deposition in ethanol; (C) by plasma in liquid ethanol; (D) by CVD plasma. Reprinted with permission from [201,202,209,210]. Copyright: 2017 American Chemical Society, 2019 Elsevier, 2018 Elsevier and 2016 Springer Nature respectively.

The utilization requires NDs be dispersible in a stable colloidal suspension. This involves the ND surface chemistry and the related $\zeta$ potential which must fall in the range $-30 \mathrm{mV}$ or higher than $30 \mathrm{mV}$ to ensure the stability of the ND dispersion. Surface is a commonly used method to produce NDs with $\zeta \sim-30 \mathrm{mV}$ at $\mathrm{pH}=7$ [211]. However, the surface chemistry of NDs strongly depends on the synthesis process used. Generally application 
of high temperatures induce graphitization with loss of surface functionalization [212]. HPHT processes induce a surface chemistry mainly formed by mainly hydroxyl groups and few carboxylic groups [213]. Differently, detonation nanodiamonds possess the richer surface chemistry with respect to other carbon nanostructures composed by hydroxyl and carboxylic groups as well as epoxides and lactones [213]. If CVD plasmas are utilized to produce $\mathrm{Nds}$, generally the surface is $\mathrm{H}$ terminated. The different $\mathrm{ND}$ chemistries require a surface homogenization to obtain a uniform ND surface chemistry. Homogenization is performed either under oxidative or reductive conditions depending on the desired surface chemistry [214].

\subsection{Nanodiamond Functionalization}

Concerning the ND functionalization processes, the modification processes of the diamond surface chemistry are rather consolidated. The graphitic shell may also be removed using a mixture of $\mathrm{FeSO}_{4}$ and $\mathrm{H}_{2} \mathrm{O}_{2}$ (Fenton) which readily attack the non-diamond phases terminating the surface with $\mathrm{OH}$ groups [215]. Diamond surface hydroxylation may be obtained also via mechanical methods such as milling and ultrasounds in water causing radical reactions resulting in the $-(\mathrm{OH})$ termination [216]. Oxidation of the ND surface can be obtained during the process of ND purification involving highly oxidizing agents such as strong acids, singlet oxygen in $\mathrm{NaOH}$, strong ozone, air treated in the presence of a catalyst which are used to remove the amorphous/graphitic coating of the diamond core $[217,218]$. Surface oxidation is also performed using supercritical water at $350-450{ }^{\circ} \mathrm{C}$ [219] or using ozone [220]. Once the ND surface is oxidized, different routes are applied to obtain the desired functional groups. One route consist in the non-covalent functionalization which can be performed utilizing or electrostatic or hydrophobic interactions [221]. In the first case the electrostatic interaction is utilized to attach to the ND surface polar molecules as DNA sequences, polymeric molecules etc. The second route relies on apolar interactions utilized and based to self-assemble the desired molecules on the ND surface. This chemical functionalization has been widely used in preparation of composites and drug delivery [214,222].

Non-covalent functionalization is an easy process which however suffers from poor reproducibility due to the lack of control on the adsorption process. The covalent functionalization employs carboxylic and hydroxyl groups to graft additional molecules. As an example, after activation with thionyl chloride, carboxylic groups can react with amines [208]. This reaction was utilized to obtain PEGylated NDs [223]. We observe that PEG-chains can be terminated with amino, thiol, and azido groups frequently used to selectively bind a variety of different biomolecules through covalent bonds. Another widely utilized functionalization is the derivatization of the hydroxyl groups via esterification obtained via high temperature -OH carboxylation succinic anhydride at high temperatures [224]. An alternative route for the derivatization of NDs is the use of siloxanes [213]. However, this kind of functionalization offers a scarce control of the siloxane coating thickness on the ND core which also depend on the siloxane precursor used [213]. Siloxane functionalization was successfully utilized for drug delivery, for covalently anchor dyes and receptors [225]. Other kinds of functionalization include the reaction of alkyl chlorides of hydroxylated NDs, the functionalization with alkenes of H-terminated NDs, or the use of diazonium salts [226]. Finally, carboxyl groups on the ND surface can be transformed in azido groups ready for successive click chemistry reactions [227]. Some of the more common functional groups used to change the ND surface chemistry are summarized in Figure 11. 


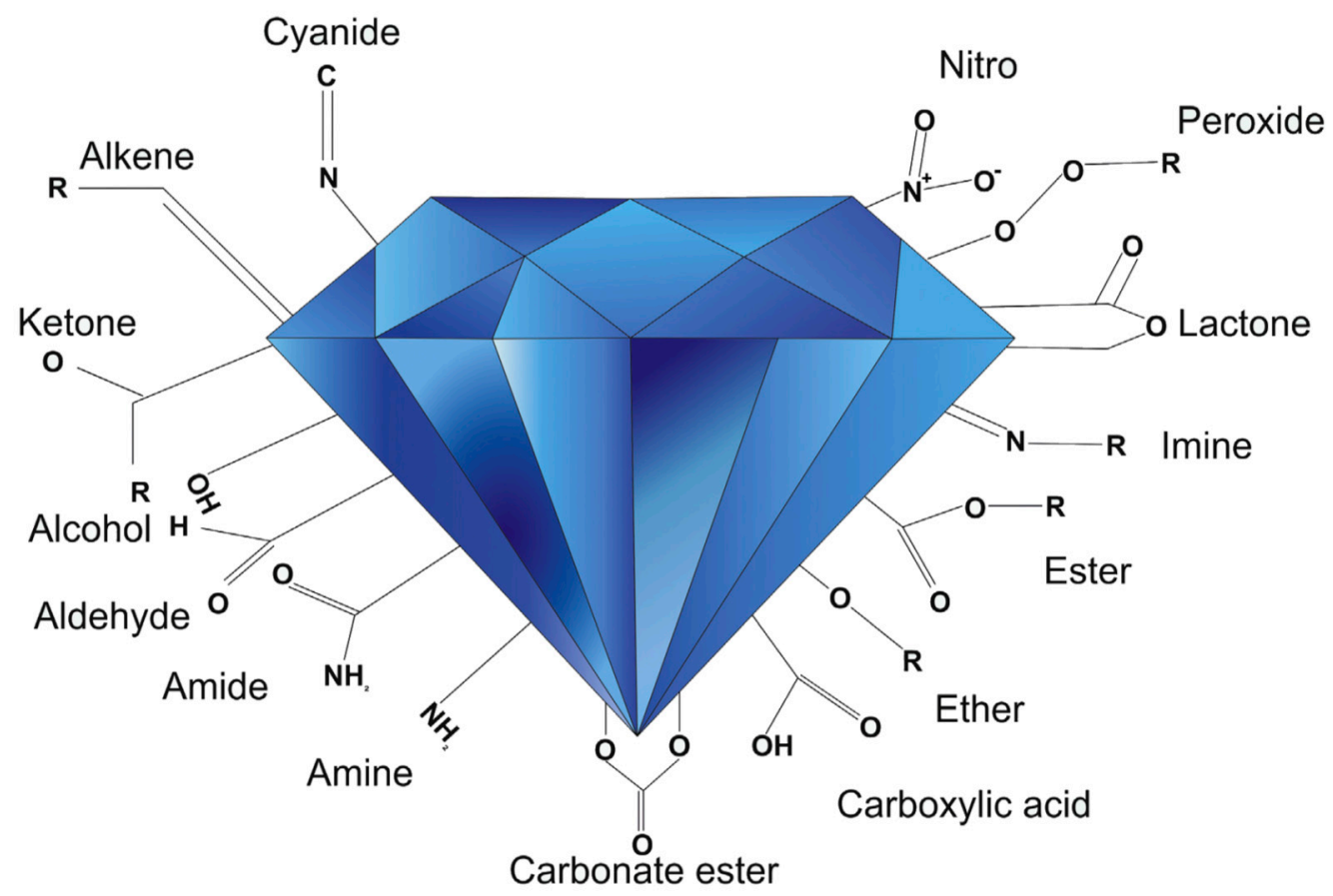

Figure 11. Modification of the surface chemistry of NDs with various functional groups. Reproduced with permission from [228]. Copyright 2019 Elsevier.

\subsection{Nanodiamond Sensing}

The possibility of synthesizing diamonds at reasonable low costs, have promoted the use of diamond in a variety of industrial applications. An example is the use of diamond to fabricate lenses for high-power high-energy radiations or for optics in harsh environments $[229,230]$. The high isolating power coupled to the outstanding thermal conductivity make diamond the material of choice for high power electrical devices [231]. Finally the superior diamond hardness and the capability to dissipate heat employed in diamond coatings for processing hard materials [232]. Diamond nanostructures are utilized in a variety of applications such as energy storage, catalysis, electroanalysis, tribology and lubrication, chromatography, and mass spectrometry [207]. In addition, one of the sectors where NDs are widely utilized is biology and medicine due to the extremely high biocompatibility. In fact, it has been shown that NDs do not influence the cell differentiation, growth and proliferation and their metabolic activity [233]. ND sensing exploits mainly its electrochemical and optical properties. With respect to sensing applications great effort was spent over the past years to develop fluorescent tags to localize individual small molecules as drugs, proteins, nucleic acids, as well as study complex biological processes [234-236]. Ideal fluorescent tags should possess the following properties: (i) high sensitivity, possibly to detect single molecules, (ii) spatial resolution at the nanoscale, (iii) high absorption coefficient and high emission quantum yield, (iv) absence of blinking and photo bleaching, (v) biocompatibility and (vi) possibility modify the original chemistry for functionalization. In the case of bio-sensing, important is also the excitation and detection fall in the "biological transparency window" thus avoiding simultaneous excitation of endogenous fluorescent molecules of blood constituents, of cofactors, and of water. Most of the fluorescent markers do not possess all of these features. NDs, owing to their unique optical and chemical properties, are proposed to be the best alternative to conventional organic dyes, quantum dots or nanoparticles if optical properties, photostability, sensitivity and biocompatibility are concerned. In particular NDs may couple fluorescence properties with quantum sensing [237] which make then an unique probe for the detection of several physical and biological entities. 
As pointed out previously, diamond emission properties are due to defects acting as color centers and emitting at different wavelengths (see Figure 12). Among them, the nitrogen-vacancy (NV) centers constituted to a substitutional nitrogen atom next to a vacancy, is the more diffuse defect. Vacancies can be created by high-energy irradiation with electrons, protons, helium ions. Then annealing at $600-800^{\circ} \mathrm{C}$ induce vacancy mobility and trapping by nitrogen atoms always present in diamond [207]. This process results in the generation of two kinds of $\mathrm{NV}$ centers: the neutral $\left(\mathrm{NV}^{\circ}\right)$ and negatively charged $\left(\mathrm{NV}^{-}\right)$characterized by emission at 575 and $637 \mathrm{~nm}$ respectively.

NE8 : 782-802 nm

$\mathrm{NiSi} \cdot 767-775 \mathrm{~nm}$

$\mathrm{Cr}=740-790 \mathrm{~nm}$

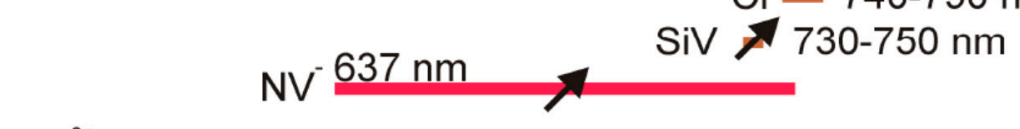

H3 503 nm

? $532 \mathrm{~nm}$

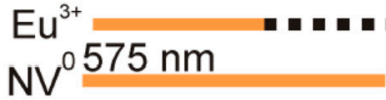

$550 \mathrm{~nm}$

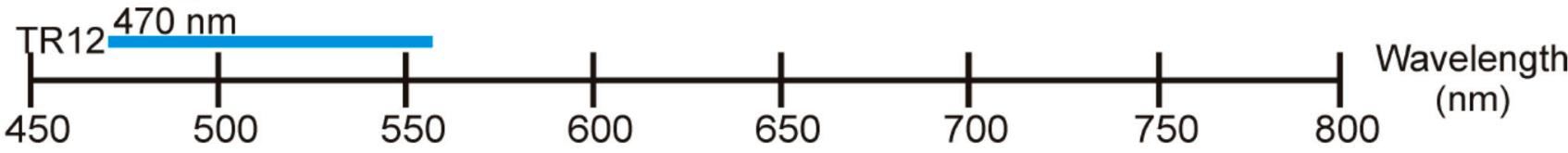

Figure 12. Spectral map of various single emitters in diamond. For centers with emission wavelengths shorter than $730 \mathrm{~nm}$, the length of the colored line represents the approximate width of the emission spectrum of the color center including phonon sidebands. The wavelength given for each center denotes the zero-phonon-line (ZPL) wavelength. For centers with emission wavelengths above $730 \mathrm{~nm}$, the labeled wavelength indicates the spread of the observed ZPL positions, while the colored line indicates the width of the ZPL for a single center. Note that especially in the near the infrared region, a multitude of unidentified color centers exists, which are not given here for clarity. Centers for which spin manipulation of single centers has been shown are labeled with a black arrow. Reprinted with permission from [238]. Free download.

As it appears from Figure 12, the $\mathrm{NV}^{-}$center is of particular interest because (i) it can be optically pumped and emits in the visible; (ii) its ground state spin value is $S=1$ and can be spin-polarized and manipulated using electron paramagnetic resonance; (iii) the $\mathrm{NV}^{-}$is characterized by a long spin coherence time. These properties have been exploited for high-resolution magnetic sensing allowing biomolecule detection [239], fluorescence resonance energy transfer [240] and biomedical imaging [241] quantum information [242], base studies in quantum optics [243].

As for sensing, the ultra high sensitivity of nitrogen vacancy was utilized to detect single protein molecules [244] and reconstruct its structure. At this aim, a sort of "quantum lock-in" was utilized to reject noise when the $\mathrm{NV}^{-}$is coupled to the protein molecule as shown in Figure 13. A microwave pulses delivered to the system with a timing in resonance with the $\mathrm{NV}^{-}$spin precession. Coupling with the external molecule causes a resonance leakage thus enabling the detection of the molecule. The diamond $\mathrm{NV}^{-}$was also used to detect substances in a solution with unprecedented sensitivity. In [245] a spectrometer detects the oscillating magnetic generated by the precession of the analyte nuclear spins weakly coupled to the diamond $\mathrm{NV}^{-}$via magnetic dipole interactions. The $\mathrm{NV}^{-}$centers are optically interrogated using a narrowband dynamical decoupling pulse sequence, leading to an $\mathrm{NV}^{-}$spin-state. 

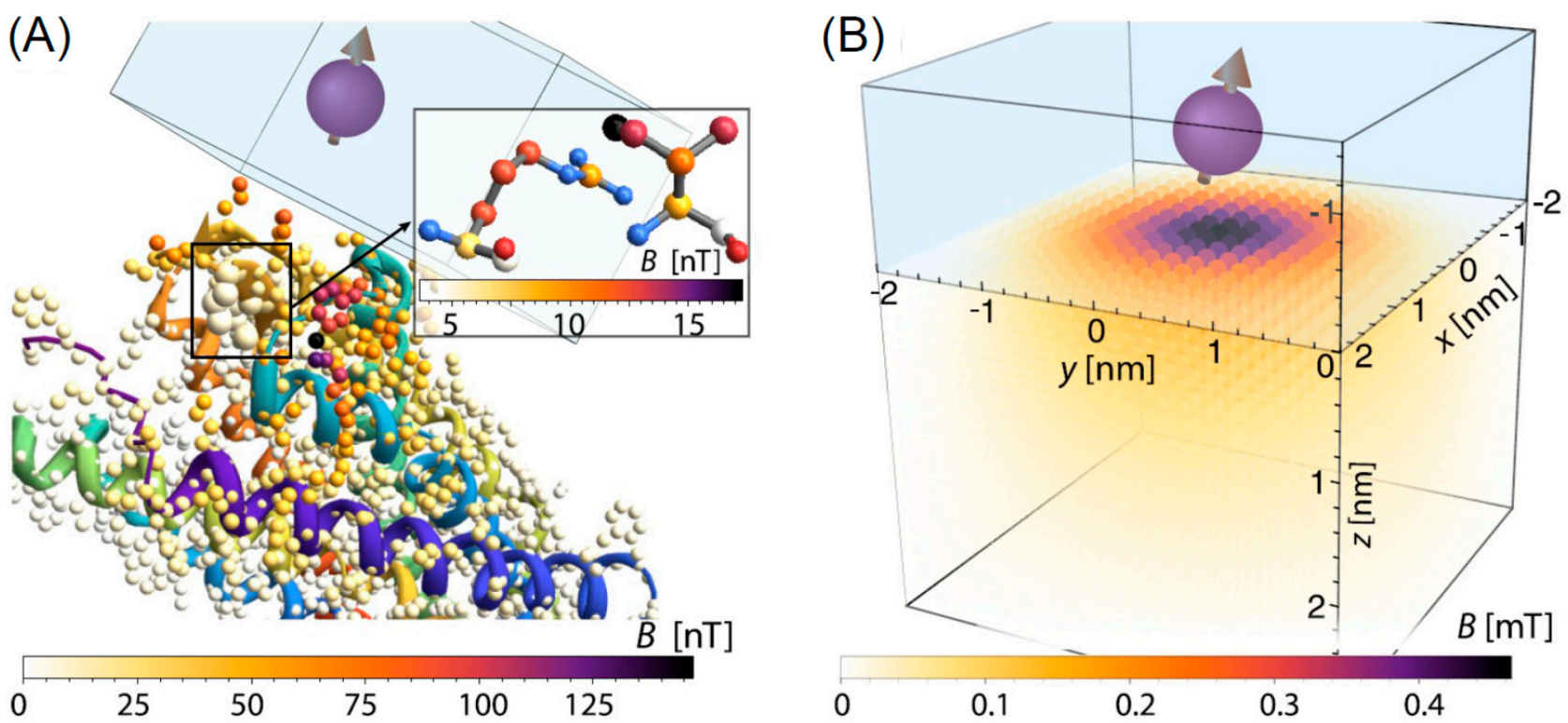

Figure 13. (A) Nuclear spin imaging with a shallow NV center in diamond. A single NV spin (purple) at 1-2 nm from the diamond surface can sense single nuclear spins in a molecule (the chemokine receptor CXCR4, ribbon diagram) anchored to the diamond. The magnetic field produced by individual ${ }^{13} \mathrm{C}$ (spheres with color scale given by $\mathrm{B}_{\perp}{ }_{\perp} / \gamma \mathrm{e}$ ) is in the range of $\mathrm{nT}$, within reach of NV sensitivity. In the inset, we show the binding site of interest [atoms other than $13 \mathrm{C}$ are blue $(\mathrm{O})$ and red (N)]. (B) A shallow NV center (2 nm from the surface) creates a magnetic field gradient $\left[\mathrm{A}(\mathrm{r})=\gamma_{\mathrm{n}}\right]$ above the [111] surface of the diamond. Note the azimuthal symmetry of the field, which causes degeneracy of the frequency shift at many spatial locations. Reprinted with permission from [244].

An interesting review on the properties and applications of the diamond NV are reported in [238]. Besides magnetic fields, the $\mathrm{NV}^{-}$is sensitive to the local electric field. Most of the techniques currently utilized to detect individual electric charges are limited to low-temperature methods such as single-electron transistors, single-electron electrostatic force microscopy and scanning tunnelling microscopy. In [246] authors demonstrate the possibility to perform high precision three-dimensional electric-field measurements using a single diamond $\mathrm{NV}^{-}$. They reached a sensitivity of $202 \pm 6 \mathrm{~V} \mathrm{~cm}^{-1} \mathrm{~Hz}^{-1 / 2}$ corresponding to the electric field generated by a single elementary charge placed at a $150 \mathrm{~nm}$ from the $\mathrm{NV}^{-}$. More recent studies promise to enhance the sensitivity in detecting the electric field produced by a local charge distribution [247] reaching a sensitivity of $150 \mathrm{mV} \mathrm{cm}{ }^{-1} \mathrm{~Hz}^{-1 / 2}$. $\mathrm{NV}^{-}$is also utilized to detect local stresses. Since at high pressure the material properties may greatly change, samples are placed in anvil cells where high pressure can be applied. However, in these experimental conditions the measure of the material's properties is very complex. In [248] authors were able to monitor the behavior of a diamond sample as a function of the temperature and the pressure applied reaching a sensitivity of $\{0.023$; $0.030 ; 0.027\} \mathrm{GPa} / \mathrm{Hz}^{1 / 2}$ in agreement with the theoretical derived values. In another work authors were able to completely reconstruct the stress tensor elements from a twodimensional field of view of NV optically detected magnetic resonance spectra [249] with a sensitivity of $\sim 0.1 \mathrm{MPa}$ at $10 \mathrm{mK}$. Another useful color center of diamond is the silicon vacancy (SiV). Differently form the $\mathrm{NV}^{-}$whose emission yield is $\sim 4 \%$, the $\mathrm{SiV}$ coupling with phonons is very wea leading to an emission efficiency of $\sim 70 \%$ at room temperature. As for $\mathrm{NV}$, also $\mathrm{SiV}$ exist in a charged $\mathrm{SiV}^{-}$and neutral state $\mathrm{SiV}^{0}$ with correspondent zero phonon lines (ZPL) at $738 \mathrm{~nm}$ and $946 \mathrm{~nm}$ respectively. $\mathrm{NV}^{-}$are electron paramagnetic resonant (EPR) defects possessing a rather long coherence time allowing a spin coupling with external species [250]. Differently the SiV are not EPR active and their ZPL relaxation time is of the order of $1 \mathrm{~ns}$ at room temperature [251]. To the long coherent times of NV spins, the correspondent ZPL is rather broad due to emission in the phonon sidebands. Differently the $\mathrm{SiV}$ is narrow (at room temperature $<1 \mathrm{~nm}$ ) making it a very useful tool for 
sensing. The limited line-width of the $\mathrm{SiV}$ defects results in an almost indistinguishable photons from separate $\mathrm{SiV}^{-}$centers which do not need any additional tuning techniques for the creation of high quality excellent single photon source building block for scalable quantum networks [251].

$\mathrm{SiV}$ vacancies can be utilized to perform high precision temperature measurements also in living cells [252]. In this work, authors used the temperature dependent position of the $\mathrm{SiV}^{-} \mathrm{ZPL}$ to estimate the local temperature. The precision obtained corresponds to $\Delta \lambda / \mathrm{DT}=0.0124 \mathrm{~nm} \mathrm{~K}^{-1}\left(\left[6.8 \mathrm{GHz} \mathrm{K}^{-1}\right)\right.$ using a bulk diamond sample. Same authors used the ZPL red shift induced of nanodiamonds to measure the temperature. Because of the lower signal-to-noise ratio, the heating was modulated to increase the measurement precision leading to an uncertainty of $521 \mathrm{mK} / \mathrm{Hz}^{1 / 2}$. This precision is important to study the molecular mechanism regarding the expression of temperature-related physiological functions. An interesting review regarding the different temperature probes (including diamond) and the techniques utilized for temperature sensing in living microorganisms may be found in ref. [253]. The SiV vacancy fluorescence lies in the NIR region which with respect to NV, better matches the optical transparency window of tissues thus giving the opportunity to better analyze biological samples. As an example, in [254] $\mathrm{SiV}^{-}$were used as to label neuronal precursor cells obtaining a very high contrast versus the cellautofluorescence. SiV are bright and highly stable defects which do not suffer from photobleaching as the organic dyes. This opened the possibility to use fluorescent NDs in high resolution microscopy where photostability is highly required. For example SiV defects were utilized to perform high resolution stimulated emission depletion microscopy with a resolution better than $150 \mathrm{~nm}$ [255]. In another work fluorescent nanodiamonds (FND) were used in correlative microscopy as dual-contrast probes [256]. Images were collected using both stimulated emission depletion and transmission electron microscopy to visualize cell organelle such as mitochondria. FNDs are currently utilized to follow fast and slow events in cells thanks to their photostability and resistance to photobleaching and photoblinking. In [257] FNDs were used for tracking the protein fate within an organism as for example the low-density lipoproteins (LDLs) and Yolk lipoprotein complexes (YLC) in Caenorhabditis elegans worm during $12 \mathrm{~h}$. Confocal microscopy and FNDs were used for long-term labeling and tracking of stem cell division, proliferation, and differentiation [258]. Cell development, fate, and contribution to regenerating tissues were observed for up to 8 days without any photobleaching.

Detonation NDs are also used to follow the fate of drug molecules after delivery. The negative charge of functional groups of the DND surface can interact with the positively charged amino group of doxorubicin, a drug used in cancer therapy. Using the ND fluorescence, the release and the effect of the doxorubicin drug can be studied in vivo in animal models [259]. An excellent review on the use of nanodiamonds for biomedical applications is given in [260]. Finally, NDs can be used as electrochemical sensors. Electrochemical measurements involve the detection of different electrostatic potential of two different electrodes immersed in a solution. The potentials are induced by the charge transfer across the electrode/solution interface which depends on the material constituting the electrode. There are a few reasons why diamond shows better performances with respect to other materials: (i) diamond electrodes display a very low background signal thus allowing improved sensitivity; (ii) diamond electrodes do not show ionizable or redox active groups generally present on the surface of the electrodes and leading to distinct background peaks or a pseudo-capacitive background to the voltammetry measurements; (iii) most of the electrodes tend to show extremely large background currents at high potentials caused by the decomposition of the electrolyte. Diamond electrodes possess a much wider electrochemical window in which redox processes may be analyzed. NDs were utilized a nano-electrochemical sensors for the detection of pyrazinamide one of the most consumed antibiotics for the treatment of tuberculosis [261]. NDs were deposited on a glassy carbon electrode and led linear response in the range from $7.9 \times 10^{-7}$ to $4.9 \times 10^{-5} \mathrm{~mol} \mathrm{~L}^{-1}$ with a LOD of $2.2 \times 10^{-7} \mathrm{~mol} \mathrm{~L}^{-1}$. NDs were also used to detect monophenols and 
bisphenols pollutants avoiding problems deriving from sensor fouling. In [262] authors were able to overcome this problem using an electrode modified with boron doped nanodiamonds to detect bisphenol-A reaching a low detection limit at $5 \mathrm{nM}$. A glassy carbon modified with biochar, nanodiamonds and chitosan, was used to detect toxic metals such as cadmium and lead [263]. The sensor showed an electrochemical sensitivities of 0.42 and $\left.5.3 \mu \mathrm{A} /(\mu \mathrm{mol} \mathrm{cm})^{2}\right)$ was found for $\mathrm{Cd}$ and $\mathrm{Pb}$ respectively. In addition, the sensor showed good stability and reproducibility over a period of 30 days. A good review of the ND applications including electrochemical sensing may be found in [264]. Table 3 summarizes characteristics of ND sensing performances.

Table 3. Detection modality, analyte and detection limit of ND based sensors.

\begin{tabular}{|c|c|c|c|c|}
\hline Method & Functionalization & Analyte & Performances & Reference \\
\hline $\mathrm{NV}^{-}$- quantum sensing & - & $\begin{array}{c}\text { chemokine receptor } \\
\text { CXCR4 }\end{array}$ & Single molecule & [244] \\
\hline $\mathrm{NV}^{-}$- quantum sensing & - & TEMPOL & $\mathrm{fM}$ & [245] \\
\hline $\mathrm{NV}^{-}$- quantum sensing & - & E field & $202 \pm 6 \mathrm{~V} / \mathrm{cm} \sqrt{ } \mathrm{Hz}$ & [246] \\
\hline $\mathrm{NV}^{-}$- quantum sensing & - & E field & $150 \mathrm{mV} / \mathrm{cm} \sqrt{ } \mathrm{Hz}$ & [247] \\
\hline $\mathrm{NV}^{-}$- quantum sensing & - & Crystal stress & $\{0.023 ; 0.030 ; 0.027\} \mathrm{GPa} / \mathrm{Hz}^{1 / 2}$ & [248] \\
\hline $\mathrm{NV}^{-}$-quantum sensing & - & Crystal stress & $\sim 0.1 \mathrm{MPa}$ at $10 \mathrm{mK}$ & [249] \\
\hline $\mathrm{SiV}^{-}$ & - & Cell temperature & $521 \mathrm{mK} / \mathrm{Hz}^{1 / 2}$ & [252] \\
\hline $\mathrm{SiV}^{-}$ & - & $\begin{array}{l}\text { Cell high resolution } \\
\text { microscopy }\end{array}$ & $<150 \mathrm{~nm}$ & [255] \\
\hline $\mathrm{SiV}^{-}$ & - & $\begin{array}{l}\text { Cell high resolution } \\
\text { microscopy }\end{array}$ & $<90 \mathrm{~nm}$ & [256] \\
\hline Fluorescent ND & - & Lipoproteins tracking & Tracking duration $=12 \mathrm{~h}$ & [257] \\
\hline Fluorescent ND & - & Cell exocytosis & Analysis duration $=8$ days & [258] \\
\hline Voltammetry & - & Pyrazinamide & $2.2 \times 10^{-7} \mathrm{~mol} / \mathrm{L}$ & [261] \\
\hline Suare wave voltammetry & - & Bisphenol & $5 \mathrm{nM}$ & [262] \\
\hline Voltammetry & - & $\mathrm{Cd}$ and $\mathrm{Pb}$ ions & 0.42 and $\left.5.3 \mu \mathrm{A} /(\mu \mathrm{mol} \mathrm{cm})^{2}\right)$ & [263] \\
\hline
\end{tabular}

\section{Carbon Quantum Dots}

Among the carbon nanostructures, carbon quantum dots (CQDs) raised to the community attention for their versatile synthesis and intriguing properties. CQDs possess a carbon-based skeleton and a large amount of oxygen-containing groups on the surface making them easily dispersible in water [265]. However, the surface chemistry must be carefully adapted to enable CQD fluorescence. Besides surface chemistry also the quantum confinement rising when the CQD size is reduced to nanometric dimensions affects the electronic structure and then the CQD optical properties. As traditional semiconductor quantum dots also CQD display excellent emission properties photobleaching resistance and chemical stability. In addition, CQDs possess also good biocompatibility with low cytotoxicity, and are environmental, and biohazard friendly not always present in other quantum dots.

\subsection{Carbon Quantum Dot Synthesis}

CQDs were discovered by a top-down route consisting in cutting large carbon nanostructures such as graphite, long CNTs or micron-sized graphene patches etc. in smaller dimensions. This exfoliation/decomposition process is performed by acidic oxidation which produces carbon particulate of small dimension and, at the same time, introduces oxygen based functional groups as hydroxyl, carboxyl groups, thus making the particulate easily dispersible in polar solvents. In [266] Chinese ink precursor was oxidized using a 
mixture of $\mathrm{HNO}_{3}, \mathrm{H}_{2} \mathrm{SO}_{4}$, and $\mathrm{NaClO}_{3}$ acids. The carbon particulate was then hydrothermally treated with dimethylformamide to introduce nitrogen functional groups, sodium hydrosulfide for sulphur functional groups and sodium selenide to graft selenium. Three different kinds of carbon dots were obtained showing tunable PL improved quantum yield and lifetime with respect to conventional CQDs. Low-cost manufacturing of CQDs is performed by electrochemical exfoliation of pure graphite electrodes in different $\mathrm{pH}$ electrolytes as $\mathrm{H}_{2} \mathrm{SO}_{4}, \mathrm{NaCl}, \mathrm{NaOH}$. In [267] high purity graphite rods were exfoliated in $7 \mathrm{~mL}$ $0.1 \mathrm{M} \mathrm{NaOH}$ aqueous solution. The resulting solution was then treated adding $1 \mathrm{~mL} \mathrm{80 \%}$ hydrazine hydrate and then centrifuged and dialyzed in deionized water to wash the CQDs and adjust the solution $\mathrm{pH}$ to 7 . The resulting particulate had a dimension in the $5-10 \mathrm{~nm}$ range and was characterized by a bright yellow emission with $14 \%$ quantum yield. A topdown method is the arc discharge [268] used to vaporize a bulk carbon electrode. During the arc discharge the temperatures reached are as high as $4000 \mathrm{~K}$ leading to the formation of a high-energy plasma. Carbon vapors condense on the opposite electrode forming carbon dots. In another experiment, CQDs were serendipitously produced in an arc discharge utilized for CNT production [269]. Fragmentation of CNTs led to the production of three kinds of CQDs with different sizes thus possessing different emission properties in the bluegreen, yellow, and orange regions. Laser ablation is another technique utilized to generate carbon gas plasma using a high energy laser pulse in organic solvents [270]. In this work natural graphite flakes suspended in a mixture of ethanol and diethylenetriamine were irradiated with a Q-Switch ND:YAG laser system. The condensation of the laser-induced plasma plume led to the formation of $\mathrm{N}$ doped graphene-like quantum dots with size of about $6 \mathrm{~nm}$. The three different chemical forms of nitrogen are generated by the pulsed laser deposition: pyridinic, pyrrolic, and graphitic GQDs. Presence of $\mathrm{N}$ doping results in an enhance emission with a quantum yield of $\sim 9.1 \%$. Pulse laser deposition is also used to vaporize carbon glassy particles suspended in polyethylene glycol 200 resulting in the formation of fluorescent CQDs [271]. The average dimension of the CQDs is $3 \mathrm{~nm}$ which were utilized for fluorescent imaging of healthy and cancer epithelial human cells. The CQDs showed good emission properties and photostability. Among the bottom-up techniques, the combustion [272] and microwave pyrolysis [273] are other methods to produce CQDs. These techniques ensure a due to the facile synthesis, safe for the environment, and are easy to scale-up and transfer to industry for mass production. The obtained CGDs are generally easily dispersible in water, possess a tunable PL. Interestingly in [274] a Maillard reaction was used to react glucose with a list of different amino acids [274]. The reaction was performed at $125^{\circ} \mathrm{C}$. The polymeric compound was the treated at $275{ }^{\circ} \mathrm{C}$ to obtain different kinds of CQDs displaying different optical properties upon the parent amino acid utilized. Reason for this is the different size, shape and composition of the synthesized CQDs leading to different optical properties. Microwaves were also utilized to assist a hydrothermal process. Glucose, sucrose or fructose were used as precursors to produce CQDs with an average diameter as small as $1.65 \mathrm{~nm}$ and water-soluble. The precursor was dissolved in water and heated using different microwave power and process duration. The CQDs exhibit deep ultraviolet emission of $4.1 \mathrm{eV}$. In a typical hydrothermal process, an organic precursor is dissolved in water or organic solvent. The mixture is then put in a Teflon-lined stainless steel autoclave and heated at relatively high temperature to form carbon seeding cores which then grow to form CQDs with dimensions less than $10 \mathrm{~nm}$. The hydrothermal method is very popular because it is simple, it produces an almost monosized distribution of CQDs with different surface functionalizations such as sulphur [275], nitrogen [276], oxygen containing groups [277]. Figure 14 shows SEM, TEM images of CQDs produced by different synthesis methods. 

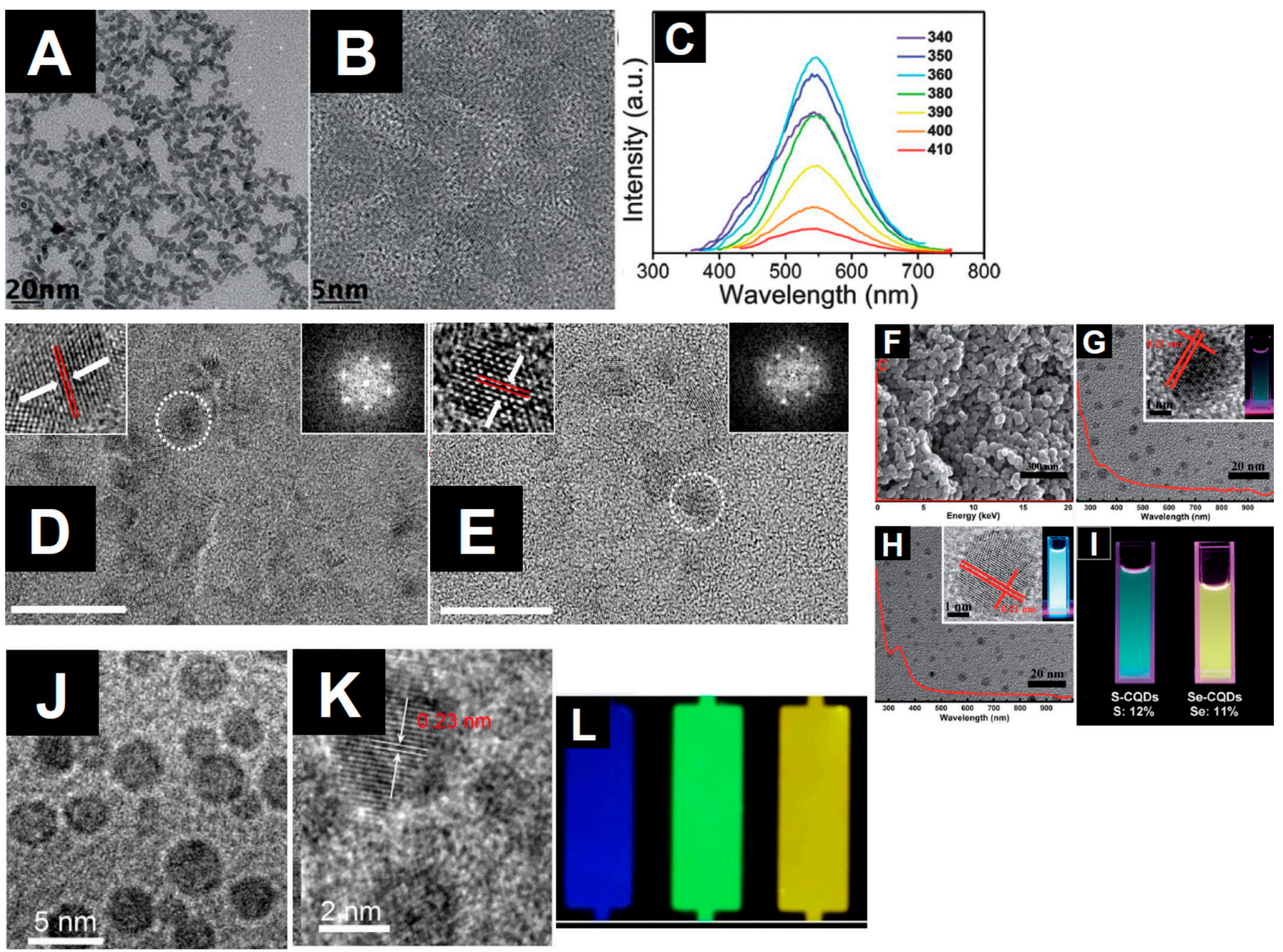

Figure 14. (A) TEM and (B) HRTEM images of the CQDs produced by exfoliation of a graphite rods in $\mathrm{NaOH}$ and deposited on freshly cleaved mica substrates. (C) PL spectra of the CQD aqueous solution at different excitation wavelengths. Reprinted with permission from [267]. Copyright 1991 Royal Society of Chemistry. (D) HR-TEM images of (E) pristine CQDs and N-doped CQDs produced by pulsed ND:YAG laser ablation of graphite flake suspension in a mixture of diethylenetriamine and ethanol. Insets show the high-quality crystalline hexagonal patterns and diffraction patterns of the CQDs and N-doped CQDs. Reproduced with permission from [270]. Copyright 2020 Elsevier. (F) FESEM image and overlapped in red EDS spectrum of CQDs from Chinese ink. (G,H) TEM images of O- (oxidized) and N- (nitrogen doped) CQDs respectively. Inset shows the high resolution of O-CQDs and N-CQDs with indicated the lattice spacing while overlapped in red are the UV-vis absorption spectra; (I) The digital images of S-CQDs and Se-CQDs emission under UV-light irradiation. Reproduced with permission from [266]. Copyright 2013 Royal Society of Chemistry. (J,K) TEM images of the Trp-CQDs of CQDs produced by reacting glucose with different kinds of amino acids and with microwave thermal treatment and (L) different emission obtained from Trp-CQDs, Leu-CQDs, and Asp-CQDs characterized by blue, green, and yellow emission. Reprinted with permission from [274]. Copyright 2014 Springer Nature.

Sulphur, chlorine, phosphor doping and their effect on the CQDs is reported in [278]. Different emission wavelengths are also obtained coordinating the CQDs with metal ions [279]. CQDs were produced polysaccharides to study the effect of $\mathrm{Cu}^{2+}, \mathrm{Sn}^{2+}, \mathrm{Cd}^{2+}$, $\mathrm{Zn}^{2+}$ ions on the CQDs optical properties. Authors found that CQD-Cu ${ }^{2+}$ ions cause an emission quenching while CQD-Sn ${ }^{2+}, \mathrm{CQD}-\mathrm{Cd}^{2+}$, and CQD- $\mathrm{Zn}^{2+}$ led to an enhancement of photoluminescence intensity. Quenching is hypothesized to derive from a photoinduced electron transfer involving the $\mathrm{Cu}$ d-orbitals and the CQD electronic configuration while in the other case the enhancement should derive from internal charge transfer between the $\mathrm{Sn}^{2+}, \mathrm{Cd}^{2+}, \mathrm{Zn}^{2+}$ ions and CQDs. Tuning the CQDs optical properties was performed by an appropriate modification of the surface chemistry. In [280], are indicated the possible approaches to redshift the emission from CQDs: (i) increasing the oxidation state of the 
surface; (ii) introduce nitrogen doping in the in the CQDs core; (iii) solvatochromism; (iv) reacting CQDs with phenylenediamines, naphthalenediamines, or trihydroxybenzene, in harsh conditions. Arginine and ethylenediamine in different proportion can be utilized to produce blue and orange light emitting CQDs as well as white light emission [280]. Finally, same authors were able to obtain important tunable electrochemical properties with the introduction of quinones in the arginine and ethylenediamine reaction mixture.

\subsection{Carbon Quantum Dot Functionalization}

The surface chemistry of the CQDs is determined by the precursor and by the synthesis process selected as previously observed. Good reviews of the CGDs synthesis and properties can be found in $[49,280-284]$.

\subsection{Carbon Dots Sensing}

The most useful and prominent property of the CQDs is their PL. Two main elements affect the optical properties of CQDs: their chemistry and the quantum confinement. CQDs display a typical absorption spectrum with two main bands at around 230 and $350 \mathrm{~nm}$ with a tail extending into the visible range. The feature at $\sim 230 \mathrm{~nm}$ is ascribed to the $\pi-\pi^{*}$ transition C-C bonds in aromatic rings, whereas the shoulder at $\sim 300 \mathrm{~nm}$ is assigned to the $\mathrm{n}-\pi^{*}$ deriving from transitions in $\mathrm{C}=\mathrm{O}$ bonds or other $\mathrm{N}$, $\mathrm{O}$ containing functional groups [285]. These last are also responsible of modification of the absorption spectra caused by the different hybridization derivatives. In addition, absorption spectra may also depend on some molecules as those obtained in citric-acid-based synthesis, displaying similar absorption spectra in the $200-400 \mathrm{~nm}$ range [286] as reported in Figure 15A. Concerning PL, the emission spectra are formed by a unique broad feature characterized by a large Stokes shift with respect to organic dyes. The position of this feature depends on the excitation wavelength which is ascribed to the presence of different dot sizes, the different surface chemistries, different emissive traps [287]. Figure 15B,C show the dependence of the absorption spectrum and of the PL spectra on the excitation wavelength and on the surface chemistry. Besides chemistry, also the presence of quantum confinement influences the optical absorption and PL properties of the CQDs [286]. PL from carbon dots may derive from the presence of stabilized surface energy traps as a result of the surface passivation which become emissive [288]. In addition, electronic states of the carbon core are involved in the PL through radiative recombination of excitons resulting from the $\pi-\pi^{*}$ transition assisted by the quantum confinement [286]. Generally quantum confinement leads to emission wavelength shortening. As the size of the CQDs decreases, the HOMO-LUMO gap increases as experimentally verified in [289] in agreement with theoretical models [290]. These properties are important to tune the emission at the desired wavelength thus enhancing the efficiency of CQDs sensing. 


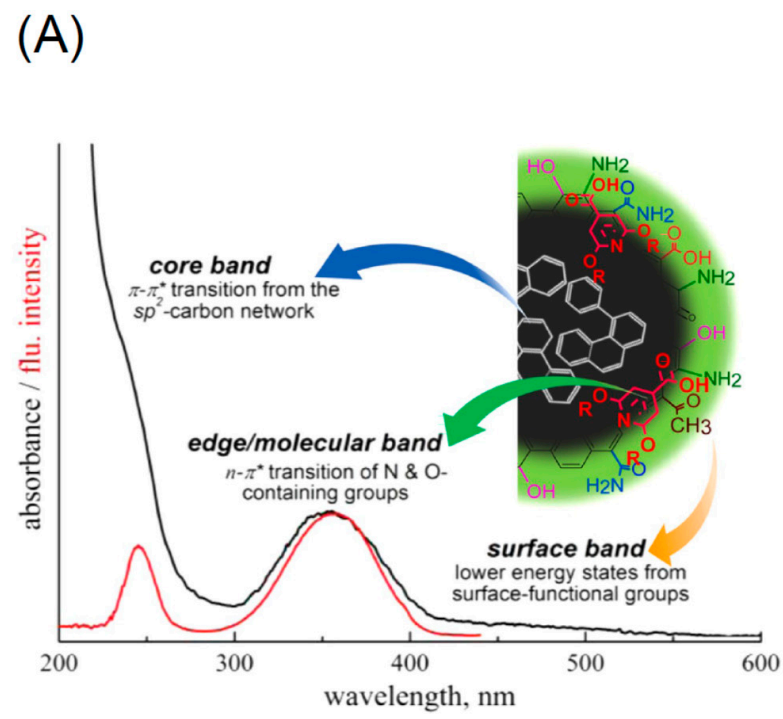

(B)

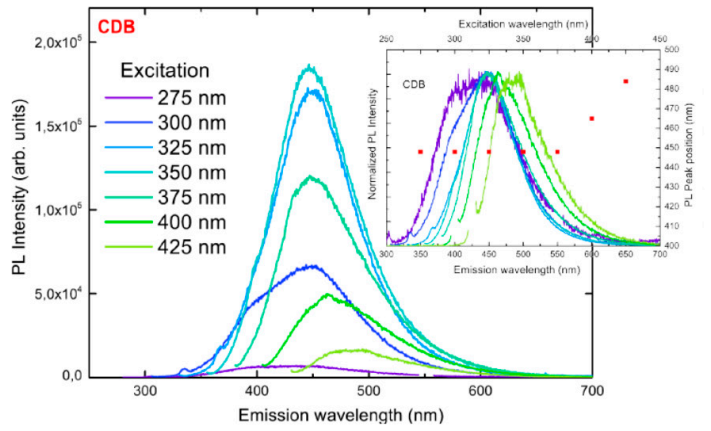

(C)

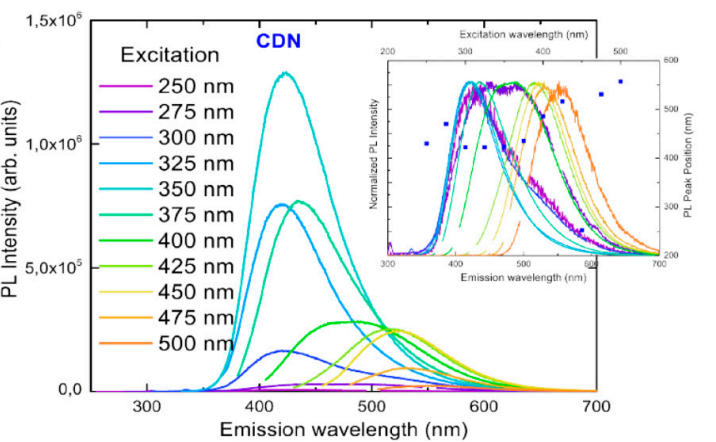

(D)

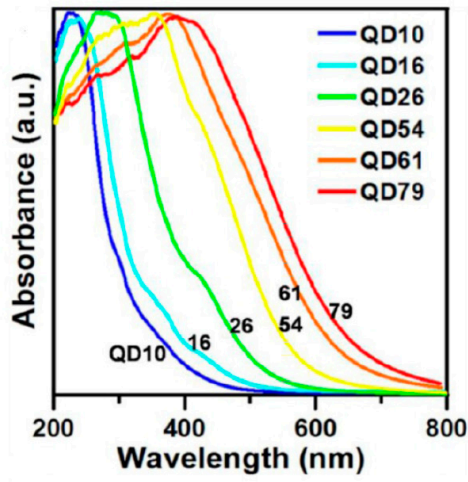

(E)

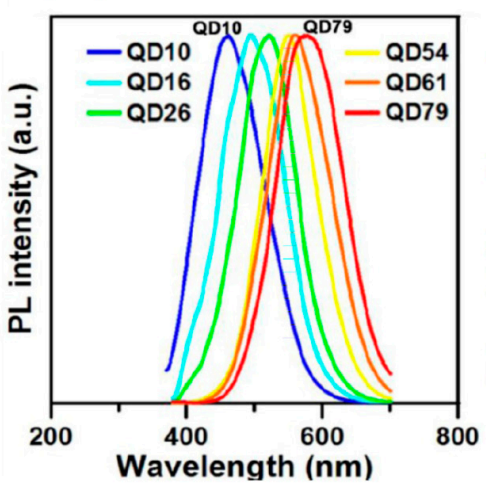

(F)

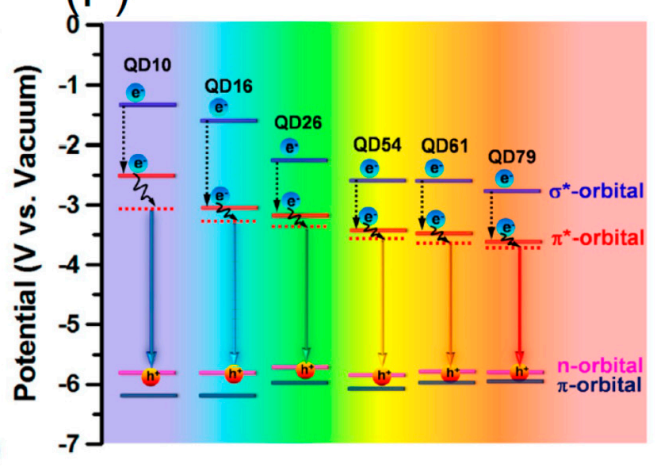

Figure 15. (A) depicts the collective explanation for the origin of PL in CDs, invoking core, edge, and surface bands. Reprinted with permission from [291]. Copyright 2017 American Chemical Society. (B) excitation PL spectra of pristine CQDs and (C) nitrogen doped CQDs. Insets show the normalized PL spectra to highlight the emission peak position on the excitation wavelength. Reprinted with permission from [292]. Copyright 2018 American Chemical Society. (D) normalized absorption and (E) normlalized PL as a function of the CQDs size. (F) dependence of the HOMO and LUMO energy levels with the CQD size. Reprinted with permission from [293]. Copyright 2016 American Chemical Society.

As a matter of fact, the fluorescent emission changes in intensity, wavelength, anisotropy, or lifetime upon interaction with different analytes and upon the analyte concentration [294]. An example is the capability to detect metal ions as $\mathrm{Hg}, \mathrm{Fe}, \mathrm{Cu}$. In this work a turn-off-turn-on of the fluorescence resonance energy transfer and ratiometric response are utilize to detect the metal ions [295] with detection limits of $10 \mu \mathrm{M}-0.2 \mathrm{nM}$ for $\mathrm{Hg}$, $1 \mu \mathrm{M}-0.58 \mathrm{pM}$ for $\mathrm{Cu}^{2+}, 17.5 \mu \mathrm{M}-2 \mathrm{nM}$ for $\mathrm{Fe}^{3+}$. In presence of the ions, an electron, charge or energy transfer occurs resulting in a selective interaction between CQDs and metal ions. The reason of the fluorescence quenching is ascribed to the functional groups on the surface of the CQDs, such as carboxyl groups, hydroxyl groups, aminogroups, etc which can selectively interact with the specific metal ions. As a consequence, the formation of a complex of metal-ion/CQDs the CQD electronic structure changes perturbing the exciton distribution and in particular increasing the non-radiative recombination processes thus leading to quenching. Another mechanism of ion metal detection is related to the change of the absorption properties of the metal-ion/CQD complex. CQD fluorescence 
quenching through metallic ions is the most utilized method to detect ferric ions. Iron assumes important roles in human physiological processes: active site of the hemoglobin and myoglobin of muscle cells. Its deficiency causes anemia reducing resistance to fatigue, cognitive problems, kidney malfunctioning and a general discomfort. Sensing iron ions is then relevant for the general wellness. The CQD fluorescence quenching induced by metal ions was exploited in [296] to detect $\mathrm{Fe}^{3+}$ ions with a LOD of 1 ppm. In this work, $\mathrm{Fe}^{3+}$ ions interact with the phenolic hydroxy groups of the CQDs, through electrontransfer between an electron in the excited state of CQD to the $\mathrm{d}$ orbital of $\mathrm{Fe}^{3+}$ leading to a nonradiative decay. CQDs are also utilized to detect $\mathrm{Hg}^{2+}$ which is one of the most poisonous and ubiquitous pollutants for the environment and health. CQDs were labeled with oligodeoxyribonucleotide. Presence of $\mathrm{Hg}^{2+}$ ions causes fluorescence quenching and a linear relation between degree of quenching and mercury concentration was found in the range 5-200 nM [297]. Fluorescence quenching was also utilized to detect $\mathrm{Cu}^{2+}$ ions. CQDs possessing surface carboxyl, hydroxyl and amine functional groups can coordinate $\mathrm{Cu}^{2+}$ ions which cause fluorescence quenching and the LOD of $23 \mathrm{nM}$ [298]. The same mechanism was also utilized to detect $\mathrm{Cr}^{6+}[299], \mathrm{Pb}^{2+}$ [300], $\mathrm{Au}^{3+}$ [301] and $\mathrm{K}^{+}$[302]. The $\mathrm{Cr}^{6+}$ was detected in a wide concentration range of $0-140 \mu \mathrm{M}$ with a LOD of $40 \mathrm{nM}$. In the case of $\mathrm{Pb}$ the $\mathrm{LOD}$ was as low as $4.5 \mathrm{ppb}$ while for $\mathrm{Au}^{3+}$ was $64 \mathrm{nM}$. Finally, for $\mathrm{K}^{+}$the fluorescent detection had a linear behavior in a wide range of $1-100 \mu \mathrm{M}$ while the LOD was $0.0570 \mu \mathrm{M}$. In the case of $\mathrm{Ag}^{+}$interaction with the CQDs led to a fluorescence enhancement [303]. This effect was attributed to the reduction of $\mathrm{Ag}^{+}$ions to $\mathrm{Ag}^{0}$ with consequent enhancing of the radiative processes. Fluorescence enhancement occurs also when the absorption bands overlap the emission bands then enabling the metal ion detection [295]. Fluorescence enhancement had a linear behavior in a concentration range of $0-90 \mu \mathrm{M}$ with a LOD of $320 \mathrm{nM}$.

Quenching is also used to detect anions. In a typical experiment, first the CQDs are coordinated with a metal ion to quench the fluorescence which is reactivated by adding an anion. Different couples of CQDs-metal ion/anion were tested such as CQDs$\mathrm{Cu}^{2+} / \mathrm{S}^{2}$ [304]. The turn-on sensor showed good selectivity towards other metal ions and a LOD of $1.72 \mu \mathrm{M}$. For CQDs-Cu ${ }^{2+} / \mathrm{H}_{2} \mathrm{~S}$ [305] sensing was linear with the $\mathrm{H}_{2} \mathrm{~S}$ concentration in a range of $5 \mu \mathrm{M}$ to $100 \mu \mathrm{M}$ and a LOD of $0.7 \mu \mathrm{M}$. Polyethylenimine-capped CQDs were used to detect $\mathrm{Cu}^{2+} / \mathrm{CN}^{-}$anions [306]. The sensor had a linear range from 2 to $200 \mu \mathrm{M}$ and a LOD of $0.65 \mu \mathrm{M}$. A sensor based on carboxylated CQDs detected the $\mathrm{Eu}^{3+} / \mathrm{PO}_{4}{ }^{3-}$ couple with a LOD of $5.1 \times 10^{-8} \mathrm{~mol} / \mathrm{L}$ and a linear range from $4.0 \times 10^{-7}-1.5 \times 10^{-5}$ $\mathrm{mol} / \mathrm{L}$ [307]. Finally, a colorimetric fluorescence "turn-on" sensor was obtained using amino functionalized CQDs to detect thiocyanate ions [308]. The change of color was observed by eye at a concentration of $1 \mu \mathrm{M}$ and a LOD of $0.36 \mu \mathrm{M}$ using fluorescence spectroscopy.

Another mechanism exploited for sensing is the photo-induced electron transfer. In this process, exploiting the donor-acceptor interactions an electro-deficient group binds with an electron-rich fluorophores. In the photo-induced electron transfer, the excited-state of fluorophores is likely to donate an electron to the ground-state of the analyte explosive compounds. This coupling leads to a non-radiative de-excitation of the fluorophore which lowers fluorescence intensity. This mechanism was utilized in combination with CQDs to detect explosives [309]. Moreover, the measure of the fluorescence lifetime is an indication of the coupling of CQDs to the external analyte. In [310] water soluble CQDs with optical band gap of $3.4 \mathrm{eV}$ were synthesized for bio-imaging. CQDs were used as fluorescent probes which were easily internalized in cells. Cell imaging was obtained by a two-photon excited fluorescence lifetime imaging microscopy. Using a $750 \mathrm{~nm}$ femtosecond laser excitation was utilized to get long fluorescence lifetime, high-contrast resolution images which coupled to the low CQD cytotoxicity make them interesting for biological applications. As for this kind of applications, CQDs are utilized as electrochemical sensors to detect important neurotransmitters as tyrosine, epinephrine, norepinephrine, acetylcholine, serotonin and in particular dopamine. Here we will give only some examples while a more 
extended description may be find in [282]. A glassy carbon electrode was modified with CQDs to sense dopamine [311]. The sensor provided an oxidation peak current linear with the dopamine concentration in the range from $0.1 \mu \mathrm{M}$ to $30.0 \mu \mathrm{M}$ with the LOD as $11.2 \mathrm{nM}$. Another dopamine sensor was fabricated using reduced graphene oxide-CQDs (rGO-CQDs) [312]. rGO-CQDs showed better electrochemical responses when detecting dopamine if compared to simple glassy carbon electrode or glassy carbon electrodes modified with CQDs or graphene oxide-CQDs in terms of linearity of the peak current and sensitivity which ranges between 0 and $10^{-3} \mathrm{M}$ and a low LOD of $1 \mathrm{nM}$. In another study, CQDs were used to detect acetylcholine [313]. A layered flower-like formed by NiAl and its hydroxide displaying positive surface charge was decorated with negatively charged CQDs. The structure exhibited enhanced electroconductivity and electrocatalytic performance for acetylcholine oxidation. The sensor showed linear response in the concentration range from 5-6885 $\mu \mathrm{M}$ with a low LOD of $1.7 \mu \mathrm{M}$. An electrochemical sensor based on $\mathrm{N}$ doped graphitic CQDs was synthesized to detect epinephrine [314]. The tests were carried out using cyclic voltammetry in presence of in the presence of $100.0 \mathrm{mM}$ pyrrole and of $25.0 \mathrm{mM}$ epinephrine. The electrode was highly performant with a broad linear range from $1.0 \mathrm{pM}$ to $1.0 \mathrm{nM}$ and a LOD of $3 \times 10^{-13} \mathrm{M}$. CQDs are extensively used to detect small molecules of rather different nature including biomolecules as glucose involved in diabetes pathology, through fluorescence quenching of boronic acid coordinated CQDS [315]. The sensor showed a linear behavior in the range from $0.1 \mathrm{mM}$ to $10 \mathrm{mM}$ with a LOD of $5.0 \mu \mathrm{M}$. Sensing glucose is important because its unbalance in metabolism causes diabetes. Thiols as glutathione and cysteine are often involved in cellular metabolism and detoxification, are detected through $\mathrm{Cu}$, Au turn off fluorescence, while $\mathrm{S}$ via a turn-on process induced by complexation of ion metals with thiols [316] or Ag for detecting cysteine following the same principle [317]. CQDs are also utilized to detect drug molecules [318] through fluorescence enhancement, vitamin $B_{12}$ (linear behavior from 1 to $12 \mu \mathrm{g} / \mathrm{mL}$ and $\mathrm{LOD}=0.1 \mu \mathrm{g} / \mathrm{mL}$ ) [319] or proteins as hemoglobin (linear behavior from 0.05 to $250 \mathrm{nM}$ and LOD $=30 \mathrm{pM}$ ) [320] or DNA. In this last case, a turn-off-turn-on process based on methylene blue molecules is used to sense DNA sequences [321]. The surface of CQDs was functionalized with methylene blue which quenches the CQDs fluoresce via an electrontransfer process. DNA can bind methylene blue molecules which are removed from the CQDs thus restoring the fluorescence. The sensor had a linear response in the range from $3.0 \mu \mathrm{M}$ to $80 \mu \mathrm{M} / \mathrm{L}$ with a detection limit of $1.0 \mu \mathrm{M} / \mathrm{L}$. CQDs are also used to discover presence of contaminants [322]. CQDs were recently used to sense trichlorophenol in the red wine and water. The sensing is based on the interaction of trichlorophenol with the aromatic rings of the CQD core leading to an enhancing of the emission from CQDs [323]. The sensor had a linear detection in the concentration range $0.1-20 \mu \mathrm{g} / \mathrm{mL}$ with a LOD of $0.07 \mu \mathrm{g} / \mathrm{mL}$. More information can also be found in [324]. Finally, because of the biocompatibility, one of the more prominent uses of the CQDs is the fluorescent tag for bioimaging. Besides biocompatibility, CQDs possess outstanding properties as the possibility to tailor their surface chemistry/functionality, their superior photostability and high brightness, and spontaneous penetration capabilities which explain the excellent potential of CQDs as probes for the study of biological systems, and for imaging-guided biomedical applications. Over the past decade, applications of CDs in bioimaging were achieved in two broad categories: in vitro imaging of cells and cell organelles, and in vivo applications mainly devoted to drug delivery and visualization of CQDs biodistribution [265,324]. In in vitro cell imaging CQDs are used to enlighten specific structural elements or biological parameters. As an example, CQDs functionalized with both $\mathrm{N}$ and $\mathrm{Cl}$ based functional groups were utilized to detect the intracellular $\mathrm{pH}$ in HeLa cells [325]. As a matter of fact, such CQDs exhibit a fluorescence which decreases with increasing the $\mathrm{pH}$ of the medium. Authors found that the fluorescence lifetime in the $\mathrm{pH}$ range of 2.6-8.6. In another work CQDs were used to sense both $\mathrm{pH}$ and the cytochrome $\mathrm{C}$ released from mitochondria and leading to cell death. CQDs rich of hydroxyl and amine groups exhibited an average particle size of ca. $3.88 \mathrm{~nm}$ and a strong $\mathrm{pH}$-sensitive fluorescent emission which is quenched at increasing 
$\mathrm{pH}$ values. In addition, dexamethasone is known to induce the release of cytochrome $\mathrm{C}$ into the interior of HeLa cells. It was found that the fluorescence intensity of doped CQDs was strictly correlated to the cytochrome $C$ concentration [326]. The fluorescence turn-off allowed detection of cytochrome $C$ within in a range of $10-500 \mathrm{mg} / \mathrm{L}$ with a detection limit of $3.6 \mathrm{mg} / \mathrm{L}$. In another study [327], an ultrasensitive aptamer-CQDs was synthesized for sensing CA125 which is used in the clinical diagnosis as a marker of several cancers and, presently, is the best serum-based tumor marker for ovarian cancer. Gold nanoparticles are modified adding a PAMAM-Dendrimers on the surface. This dendrimer contains $\mathrm{NH}_{2}$ active sites to attach the CA125 antibody. These nanoparticles are able to capture the CA125 cancer marker and at the same time the $\mathrm{Au}$ is able to quench the aptamer-CQDs fluorescence. By measuring of fluorescence resonance energy transfer (FRET) signals between $\mathrm{CDs}$ and AuNPs as nanoquenchers, the decreasing fluorescence can be correlated to CA125 concentration. The immunosensor exhibited an extremely low calculated LOD of $0.5 \mathrm{fg} / \mathrm{mL}$ with a wide linear range from $1.0 \mathrm{fg} / \mathrm{mL}$ to $1.0 \mathrm{ng} / \mathrm{mL}$ of CA 125 . CQDs were utilized for stem cell imaging which is important in regenerative medicine to study the progression of the cell growth and modification in specific tissues such as bone, skin, and neural. The small size and biocompatibility of the CQDs ensures them to be easily internalized in stem cells via endocytosis in a concentration- and time-dependent way. Thanks to the excellent photostability, the CQDs were used to label three different kinds of stem cells without evidence of any adverse or toxic effect providing the option for a long-term imaging [267]. Similarly CQDs were developed as a fluorescent neural tracer based on cholera toxin B (CTB-CQDs), which could be internalized and transported by neurons in the peripheral nervous system of rats [328].

Results suggested that CTB-CQDs could bind with high affinity to monoganglioside GM1 and within four days were retrogradely transported from axonal terminals to neuronal soma. CQDs can serve to stain specific part of the cells. Thanks to their small sizes, surface positive charges and dopamine-mimicking properties nitrogen doped CQDs were successfully used to image the cell nucleus of four different types of cancer model cells, including rat PC12, A549, HepG2 and MD-MBA-231 [329]. CQDs can also be utilized to stain parts of cells and organelle as mitochondria [330], lysosomes [331]. Figure 16 schematize the surface functionalization and the optical properties CTB-CQDs as staining agent in in vitro and in vivo experiments.

Finally, in vivo studies have benefited from using CQDs to study if they induce chronic toxicity, their stability, their biodistribution and possible accumulation in organs/tissues. Near infrared fluorescent CQDs were developed to study the biodistribution in mice. Results put forward that the O-functionalized CQDs were firstly accumulated in the reticuloendothelial system and kidneys but subsequently they were gradually cleared via renal and fecal pathways with low toxic effects [332]. In another study CQDs were utilized to image glioma tissues taking advantage of the enhanced permeation retention [333]. The hydrophilic character of the CQDs is supposed to be one of the reasons for the major accumulation in the glioma. Moreover, the average size of the CQDs in the range of 5-10 nm permits a good infiltration of the glioma tissue being characterized by a pore size in the blood tumor barrier of $\sim 12 \mathrm{~nm}$. Experiments show also an accumulation of the CQDs in liver and kidney indicating that CQDs are mostly eliminated by these two organs. Concerning accumulation and clearance, other authors studied the CQDs biodistribution and uptake in rats and tumor mice [334]. They studied the different biodistribution of silicon- and carbon-based dots through radioelement labelling and dynamic experiments of positron emission tomography. Results indicate a rapid renal clearance from the in vivo systems for both variants of the nanoparticles. However, marked differences in the biodistribution and pharmacokinetic properties of Si- and C-dots were observed. Depending on the surface charge, positive zeta potentials and hydrophilicity induced by the surface functionalities, different accumulation in liver and intestine are obtained. An example of in vitro and in vivo use of the CQDs is represented in Figure 17A,B where CQDs could distribute only in the cytoplasm of PC12 cells, without accumulating 
on the cell membrane or in the cell nucleus. In Figure 17B CQDs are colocalized with lysosomes while in Figure 17C in an in vivo experiment, a strong fluorescence signal is obtained after injection of the CQDs on the back of a nude mouse.
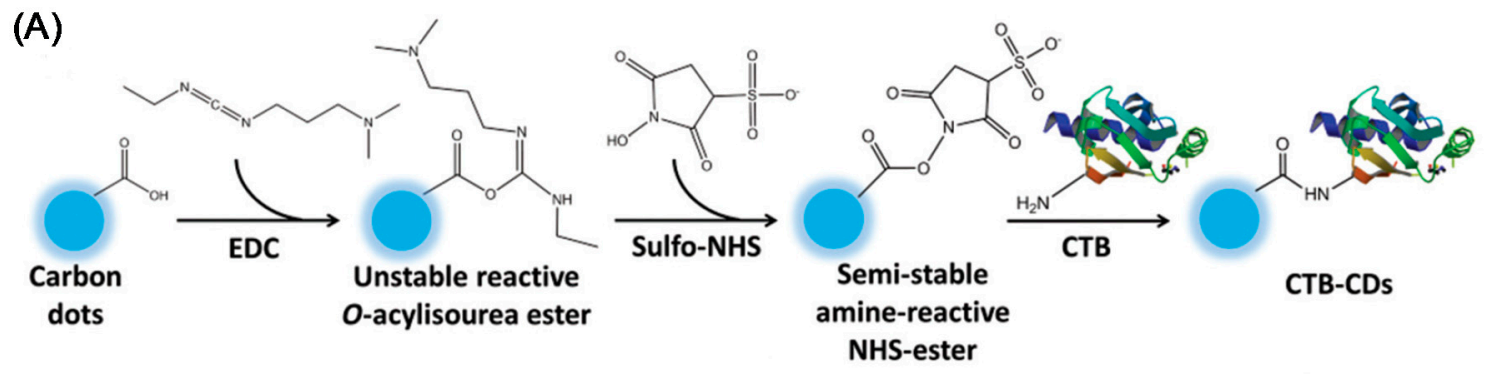

(B)

(C)
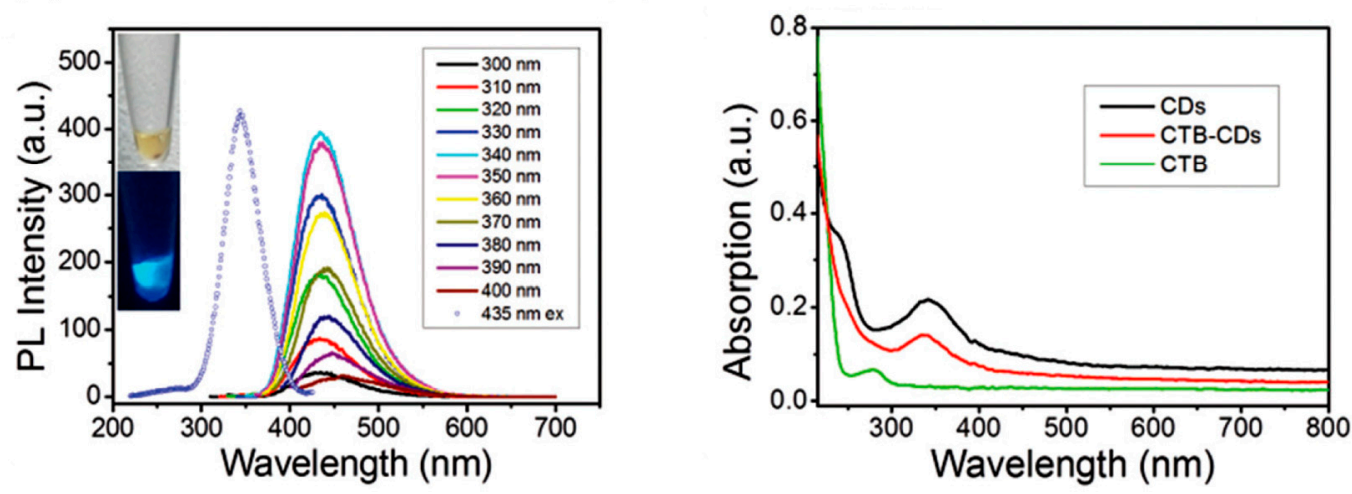

Figure 16. Preparation and characterization of CTB-CQDs conjugates. (A) Schematic illustration of the synthesis of CTBCQDs conjugates using the EDC/NHS reaction: 1-ethyl-3-(3-dimethylaminopropyl) carbodiimide hydrochloride (EDC) was used to react with the carboxyl groups on the surface of CDs, forming an unstable reactive O-acylisourea intermediate. Subsequently, N-hydroxysulfosuccinimide (Sulfo-NHS) was added to convert it to a semi-stable amine-reactive NHSester. The amine groups of CTB then covalently conjugated with the NHS-ester modified CDs to produce CTB-CDs. (B) photoluminescence (PL) spectra of CTB-CDs (Ex: excitation; Em: emission) and insets show photographs of CTB-CDs in aqueous solution under visible (upper) and UV (lower) light. (C) UV-vis absorption spectra of CDs, CTB, and CTB-CDs Reprinted with permission from [328]. Copyright 2009 RSC Publications.
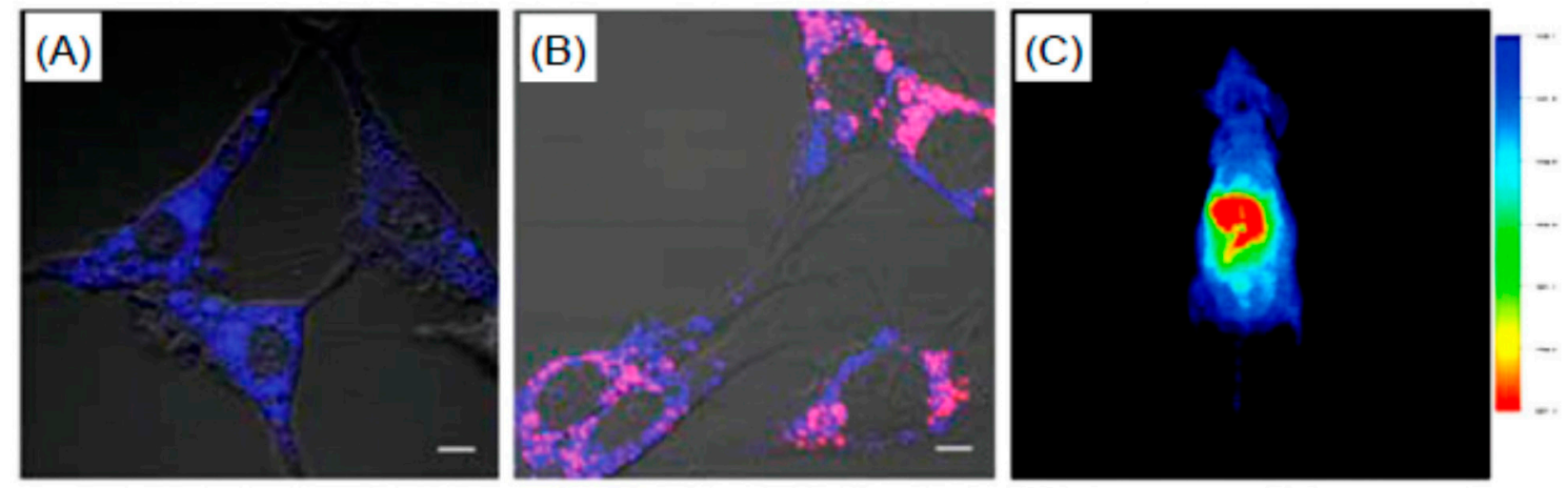

Figure 17. (A) UV-vis absorption spectra of CQDs, CTB, and CTB-CQDs; In vitro and in vivo bioimaging of CTB-CQDs: (D) NGF-treated PC12 cells were labeled with CTB-CQDs; (B) CTB-CQDs and Lysotracker; (C) CTB-CQDs were injected on the back of BALB/c nude mice. Reprinted with permission from [328]. Copyright 2009 Royal Society of Chemistry. 
CQDs may also be utilized to image the drug delivery. A nanocarrier based on cisplatin(IV) prodrug-loaded and charge-convertible CQDs (CQDs-Pt(IV)) was designed for imaging-guided drug delivery [335]. An anionic polymer with dimethylmaleic acid used to coat the CQDs-Pt(IV), displayed a charge conversion in mildly acidic tumor $(\mathrm{pH}=6.8)$ extracellular microenvironment. In addition, the positive charges of the nanocarried facilitated coupling with the negative cell membranes and the internalization. In vivo experiments showed an high tumor-inhibition efficacy and low side effects of the CQDs-Pt(IV) nanocarriers. In another study [336] a turn-on fluorescent CQDs was used as a theranostic nanoprobe. The dots were formed by assembling a polyethylenimine modified carbon dot with hyaluronic acid conjugated with doxorubicin (P-CQDs/HA-Dox). The functionalized P-CQDs/HA-Dox served as multipurpose probes for hyaluronidase detection, self-targeted imaging and drug delivery. Results showed that the nanoprobes could specifically target CD44 receptors overexpressed on many cancer cells thus enhancing the internalization. Once penetrated in the cells, hyaluronidase is activated leading to fragmentation of the HA-Dox, causing the release of Doxorubicin and the recovery of the P-CQDs fluorescence. The efficient release of Doxorubicin induced apoptosis of HeLa cells, as confirmed by MTT assay with effective treatment of cancer. Solid tumor tissues are characterized by hypoxia which remarkably reduces the efficiency of the photodynamic therapy. To solve this problem, carbon nitride was utilized to dope CQDs to induce water splitting under irradiation red light because carbon nitride increase the absorption in the red spectral region [337]. Then protoporphyrin photosensitizer, a tumor-targeting Arg-Gly-Asp motif and polyethylene glycol as a linker molecule were used to assemble PC_CN_CQDs. Results of in vitro studies show that PC_CN_CQDs succeed in increasing the $\mathrm{O}_{2}$ concentration in the cell compartments and, under light irradiation, the production of reactive oxygen species in both hypoxic and normoxic environments. The use of PC_CN_CQDs in cell viability assays showed their ability in reversing the hypoxia-triggered PDT resistance thus leading to a good growth inhibition of cancer cells.

In vitro study showed that $\mathrm{PCCN}$, thus obtained, could increase the intracellular $\mathrm{O}_{2}$ concentration and improve the reactive oxygen species generation in both hypoxic and normoxic environments upon light irradiation. Cell viability assay demonstrated that PCCN fully reversed the hypoxia-triggered PDT resistance, presenting a satisfactory growth inhibition of cancer cells in an $\mathrm{O}_{2}$ concentration of $1 \%$. In vivo experiments also indicated that PCCN had superior ability to overcome tumor hypoxia. The use of water splitting materials exhibited great potential to improve the intratumoral oxygen level and ultimately reverse the hypoxia-triggered PDT resistance and tumor metastasis. CQDs were also utilized to cross the blood brain barrier (BBB) and accumulate in the brain. This property was utilized to enhance the efficiency of drug delivery without harming the BBB integrity using CQDs [338]. Amphiphilic yellow-emissive, $3 \mathrm{~nm}$ sized CQDs were synthesized using citric acid and o-phenylenediamine. This resulted in the presence of both primary amine and carboxyl groups enabling the bioconjugation with small drug molecules. Interestingly, the amphiphilicity and the BBB penetration ability were preserved after coating CQDs with different hydrophilic molecules. Experiments showed the ability of CQDs to enter cells and inhibit the overexpression of human amyloid precursor protein and $\beta$-amyloid which is a major factor responsible for the Alzheimer disease. In another study, neuroactive properties of fluorescent $C$-dots obtained from $\beta$-alanine were synthesized to study their effects on the key characteristics of GABA- and glutamatergic neurotransmission in isolated rat brain nerve terminals [339]. Authors found that CQDs act in dose-dependent manner: (i) decreasing the exocytotic release of [3H] GABA and L-[14C] glutamate; (ii) reducing the acidification of synaptic vesicles; (iii) reducing the initial velocity of $\mathrm{Na}^{+}$-dependent transporter mediated uptake of $[3 \mathrm{H}] \mathrm{GABA}$ and L-[14C] glutamate, and (iv) increasing the ambient level of nerve neurotransmitters without significant changes of the potential of the plasma membrane of nerve terminals. Fluorescent CQDs with their neuromodulatory properties pave their potential usage for labeling/imaging key processes in nerve 
terminals with interesting theranostic perspectives. Table 4 summarizes characteristics of CQDs sensors.

Table 4. Detection modality, analyte and detection limit of CQDs based sensors.

\begin{tabular}{|c|c|c|c|c|}
\hline Method & Functionalization & Analyte & Performances & Reference \\
\hline FRET & - & $\mathrm{Hg}^{2+}, \mathrm{Cu}^{2+}, \mathrm{Fe}^{3+}$ & $\begin{array}{l}\mathrm{Hg}^{2+}: 10 \mu \mathrm{M}-0.2 \mathrm{nM} \\
\mathrm{Cu}^{2+}: 1 \mu \mathrm{M}-0.58 \mathrm{pM} \\
\mathrm{Fe}^{3+}: 17.5 \mu \mathrm{M}-2 \mathrm{nM}\end{array}$ & [295] \\
\hline Fluorescence turn-off & - & $\mathrm{Fe}^{3+}$ & $1 \mathrm{ppm}$ & [296] \\
\hline Fluorescence turn-off & Oligodeoxyribonucleotide & $\mathrm{Hg}^{2+}$ & $5-200 \mathrm{nM}$ & [297] \\
\hline Fluorescence turn-off & Carboxyl, hydroxyl and amine & $\mathrm{Cu}^{2+}$ & $23 \mathrm{nM}$ & [298] \\
\hline Fluorescence turn-off & $\mathrm{N}$ doping & $\mathrm{Cr}^{6+}$ & $40 \mathrm{nM}$ & [299] \\
\hline Fluorescence turn-off & Carboxyl, hydroxyl & $\mathrm{Pb}^{2+}$ & $4.5 \mathrm{ppb}$ & [300] \\
\hline Fluorescence turn-off & $\mathrm{N}$ doping & $\mathrm{Au}^{3+}$ & $64 \mathrm{nM}$ & [301] \\
\hline Fluorescence turn-off & $\mathrm{O}, \mathrm{N}$ functionalities & $\mathrm{K}^{+}$ & $0.0570 \mu \mathrm{M}$ & [302] \\
\hline Fluorescence enhancement & Carboxyl, hydroxyl & $\mathrm{Ag}^{+}$ & $320 \mathrm{nM}$ & [303] \\
\hline Fluorescence turn-on & Carboxyl & $\mathrm{S}^{2-}$ & $1.72 \mu \mathrm{M}$ & [304] \\
\hline Fluorescence turn-on & Carboxyl & $\mathrm{H}_{2} \mathrm{~S}$ & $0.7 \mu \mathrm{M}$ & [305] \\
\hline Fluorescence turn-on & Polyethylenimine & $\mathrm{CN}^{-}$ & $0.65 \mu \mathrm{M}$ & [306] \\
\hline Fluorescence turn-on & Carboxyl & $\mathrm{PO}_{4}{ }^{3-}$ & $15 \mu \mathrm{M} / \mathrm{L}$ & [307] \\
\hline Fluorescence turn-on & Amine & $\mathrm{SCN}^{-}$ & $0.36 \mu \mathrm{M}$ & [308] \\
\hline Fluorescence lifetime & $\mathrm{O}, \mathrm{N}$ functionalities & Cell imaging & - & [310] \\
\hline Voltammetry & Carbonyl, hydroxyl/chitosan & Dopamine & $11.2 \mathrm{nM}$ & [311] \\
\hline Pulse voltammetry & $\mathrm{GO} / \mathrm{CQDs}$ & Dopamine & $1.5 \mathrm{nM}$ & [312] \\
\hline Cyclic voltammetry & $\mathrm{NiAl} / \mathrm{CQDs}$ & Acetylcholine & $0.14 \mu \mathrm{M}$ & [313] \\
\hline Voltammetry & $\mathrm{N}$ doping & Epinephrine & $3 \times 10^{-13} \mathrm{M}$ & [314] \\
\hline Fluorescence turn-off & Boronic acid coordinated CQDS & Glucose & - & [315] \\
\hline Fluorescence enhancement & $\mathrm{N}$ doping & Amoxicillin & - & [318] \\
\hline FRET & O functionalities & Vitamin $B_{12}$ & $0.1 \mu \mathrm{g} / \mathrm{mL}$ & [319] \\
\hline FRET & Carboxyl and amine & Hemoglobin & $30 \mathrm{pM}$ & [320] \\
\hline Fluorescence turn-on & Methylene blue/CQDs & DNA & $1.0 \mu \mathrm{M} / \mathrm{L}$ & [321] \\
\hline Fluorescence enhancement & $\mathrm{S}, \mathrm{N}$ doping & Trichlorophenol & $0.07 \mu \mathrm{g} / \mathrm{mL}$ & [323] \\
\hline FLIM & $\mathrm{N}, \mathrm{Cl}$ & $\mathrm{pH}$ & - & [325] \\
\hline Fluorescence turn-off & Hydroxyl, amine & $\mathrm{pH}$, cyt C & $3.6 \mathrm{mg} / \mathrm{L}$ for cyt $\mathrm{C}$ & [326] \\
\hline Fluorescence turn-off & Au-PAMAM/CQDs & CA125 & $0.5 \mathrm{fg} / \mathrm{mL}$ & [327] \\
\hline Imaging & $\mathrm{N}$ doping & $\begin{array}{l}\text { C12, A549, HepG 2, and } \\
\text { MD-MBA-231 cells }\end{array}$ & - & [329] \\
\hline Imaging & O functionalities & $\begin{array}{l}\text { Reticuloendothelial system } \\
\text { and kidneys }\end{array}$ & - & [332] \\
\hline Imaging & $\mathrm{N}$ doping & Glioma tissues & - & [333] \\
\hline Imaging & CQDs/Pt(IV) & Drug delivery & - & [335] \\
\hline Imaging & $\begin{array}{l}\text { Polyethylenimine/CQDs- } \\
\text { hyaluronic/Doxorubicin }\end{array}$ & $\begin{array}{l}\text { Hyaluronidase detection, } \\
\text { self-targeted imaging and } \\
\text { drug delivery }\end{array}$ & - & [336] \\
\hline Photodynamic theraphy & $\begin{array}{c}\text { PEG-Arg-Gly-Asp } \\
\text { motif-protoporphyrin-carbon } \\
\text { nitride/CQDs }\end{array}$ & Solid tumor tissues & - & [337] \\
\hline Crossing the blood brain barrier & - & B-amyloid targeting & - & [338] \\
\hline Imaging & $\mathrm{N}$-functionalities & $\begin{array}{l}\text { GABA- and glutamatergic } \\
\text { neurotransmission }\end{array}$ & - & [339] \\
\hline
\end{tabular}

FRET = fluorescence resonance energy transfer. FLIM = Fluorescence Lifetime Imaging Microscopy. 


\section{Carbon Nanotubes}

Carbon Nanotubes (CNTs) is a one-dimensional form of carbon possessing has a perfect hollow cylindrical shape. CNTs can be considered as a graphene sheet (an hexagonal network of carbon atoms) rolled up along certain directions corresponding to the CNT chirality. Rolling up a single graphene layer leads to a single walled SWCNT while rolling up multiple graphene sheets results in a multiwalled CTN (MWCNT). Similarity of CNT to graphene regards not only their geometrical structure but also their electronic properties. As in graphene, also in CNTs the carbon atoms are in $\mathrm{sp}^{2}$ hybrids forming three bonds along directions separated by $120^{\circ}$ with three neighboring carbon atoms. This structural configuration leads to the generation of hexagonal rings and to a honeycomb lattice. CNTs and graphene share many interesting properties. The strong carbon-carbon bonds are responsible for the high mechanical strength of the SWCNTs have strong possessing a Young's modulus value range from 320 to $1470 \mathrm{GPa}$ and breaking forces ranging from 13 to $52 \mathrm{GPa}$ [340]. Concerning the electrical properties, CNTs display semiconducting or metallic character. These differences are generated by the direction selected for rolling up the graphene sheet namely the chirality [341]. This direction is defined by two indices $(m, n)$ as schematized in Figure 18. If the direction corresponds to the CNT main axis $(m=i, n=0)$ $\mathrm{i}=1,2,3 \ldots$ then a zig-zag metallic CNT is obtained, for $(\mathrm{m}=\mathrm{i}, \mathrm{n}=\mathrm{i})$ an armchair CNT is obtained while $(m=1, n=j) i \neq j, i, j=1,2,3 \ldots$ a chiral CNT is formed [342].

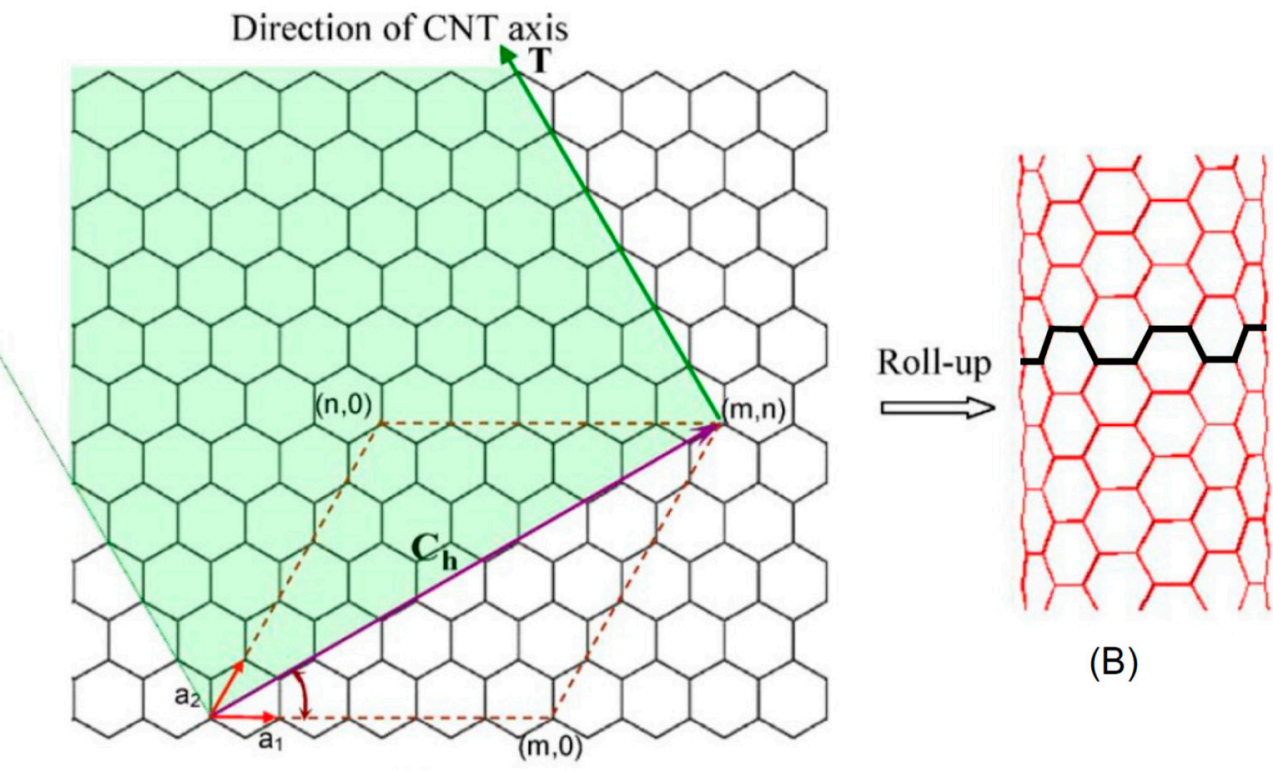

(A)

Figure 18. Roll-up vector defining the structure of carbon nanotubes. (A) Graphene lattice and (B) carbon nanotube along the indicated axis leads to an armchair CNT. Reprinted with permission from [342]. Copyright 2006 Elsevier.

It was found that when $(\mathrm{m}-\mathrm{n}=3 \mathrm{i} \mathrm{i}=1,2,3 \ldots)$ the CNT is metallic while for $(m-n=3 i \pm 1 i=1,2,3 \ldots)$ the CNT behaves as a semiconductor. The electrical conductivity along the main axis of metallic CNTs can be as high as $2 \times 10^{7} \mathrm{~S} \mathrm{~m}^{-1}$ [343], while for MWCNTs the electrical conductivity is about $2 \times 10^{5} \mathrm{~S} \mathrm{~m}^{-1}$ [344]. Besides conductivity, CNTs possess also a good thermal conductivity which for individual SWCNT was reported to be $3500 \mathrm{~W} \mathrm{~m}^{-1} \mathrm{~K}^{-1}$ [345], higher than that of bulk graphite (about $2000 \mathrm{~W} \mathrm{~m}^{-1} \mathrm{~K}^{-1}$ ), while for individual MWCNT the thermal conductivity is $\sim 3000 \mathrm{~W} \mathrm{~m}^{-1} \mathrm{~K}^{-1}$ [346]. SWCNTs possess a rather high theoretical surface area which in average is $\sim 1315 \mathrm{~m}^{2} \mathrm{~g}^{-1}$ [347] (about one half that of graphene) which however depends on the quality of SWCNTs with an highest value of $1587 \mathrm{~m}^{2} \mathrm{~g}^{-1}$ [348]. The one-dimensional tubular morphology of CNTs also bring them unique properties. CNTs have super high length-to-diameter aspect ratio: the diameter measures typically $0.4-2.5 \mathrm{~nm}$ while the length can be as high as $20-1000 \mathrm{~nm}$ 
for SWCNTs, while for MWCNTs the diameter ranges between 1.4-100 nm and the length between 1-500 $\mu \mathrm{m}$ [349]. Due to the tubular shape, CNTs possess a certain internal volume which can be used to house specific functionalities. Furthermore, the presence of curvature makes CNTs more reactive than pristine graphene and chemical functionalization of their surface may be done more easily [350].

\subsection{Carbon Nanotube Synthesis}

The broad interest in CNTs is a direct consequence of the research around fullerenes. Sumio Iijima studying the production of fullerenes using an arc evaporation system, serendipitously discovered a new for of carbon consisting in multi walls filaments of carbon with a diameter from 3 to $30 \mathrm{~nm}$ invariably closed at both ends [351]. From that moment, a large number of alternative methods have been developed for the CNTs synthesis and it became clear that two kinds of CNT could be produced: single or multi walled CNTs. As original CNT synthesis technique, the arc discharge method is well established and broadly utilized. The arc discharge apparatus consists in two highly pure graphite electrodes which are faced at a distance of 2-3 mm. One of the electrodes contains also a certain amount of metal catalysts as $\mathrm{Fe}, \mathrm{Co}, \mathrm{Ni}$, or $\mathrm{Mo}$ [352]. In the reaction chamber a $\mathrm{DC}$ arc discharge is then produced in a He atmosphere a voltage between the electrodes. As the discharge is triggered, the graphite and metal catalyst evaporate and condense onto the cathode or on the walls of the reactor forming carbon soot containing both SWCNTs and MWCNTs. Interestingly, the selection of the metallic catalyst and of the inert gas in the reactor chamber determinates whether the resultant CNTs are SWCNTs or MWCNTs [353]. The reactor atmosphere, pressure and arc current are important parameter for the process to control the yield and quality of CNTs [354]. It was shown that the final morphology of CNTs is strongly dependent on the different atmospheres used. High arc discharge currents in a $\mathrm{He}+\mathrm{CH}_{4}$ atmosphere at high pressure leads to thick nanotubes decorated with carbon nanoparticles [355].

In recent arc discharge processes the production of MWCNTs is performed without catalysts. On method is the arc-discharge under He, ethanol, acetone and hexane atmosphere at various pressures (from 150 to 500 Torr) $[44,356]$. Another possibility to synthesize MWCNTs is the arc discharge in an $\mathrm{NH}_{3}$ atmosphere at $0.02 \mathrm{MPa}$ [357]. Authors showed that there is not significant difference in the structure and shape of the obtained CNTs obtained with similar processes in $\mathrm{He}$ and $\mathrm{H}_{2}$ atmospheres. Pulsed arch discharge in air may be also utilized to produce MWCNTs on a Ni substrate as shown in [358]. More information can be found in [352].

SWCNTs are produced with arc discharges using an $\mathrm{H} / \mathrm{Ar}$ atmosphere and anodes made of graphite and a metal, such as $\mathrm{Ni}, \mathrm{Fe}, \mathrm{Co}, \mathrm{Pd}, \mathrm{Ag}, \mathrm{Pt}$, etc. or mixtures of $\mathrm{Co}$, $\mathrm{Fe}$, Ni with other elements like $\mathrm{Co}-\mathrm{Ni}, \mathrm{Fe}-\mathrm{Ni}, \mathrm{Fe}-\mathrm{No}, \mathrm{Co}-\mathrm{Cu}, \mathrm{Ni}-\mathrm{Cu}, \mathrm{Ni}-\mathrm{Ti}$ [352]. The selection of the metal catalyst will influences the SWCNT production yield. The generation of SWCNTs is due to the co-evaporation of Co and graphite in an arc discharge leading to the formation of single atom wall nanotubes with a diameter of $\sim 1.2 \mathrm{~nm}[359,360]$. Besides $\mathrm{Ni}$, one of the most utilized catalysts for SWNTs synthesis, the role of Ni, Pd, and Pt in the formation of carbon clusters was studied in [361]. More information can be found in [352,362]. The need to produce CNTs in a more controlled way led to the development of alternative synthesis methods as the laser ablation. Compared to other techniques, laser ablation is superior to produce high quality SWCNTs. Generally Nd:YAG and $\mathrm{CO}_{2}$ lasers are used to vaporize a graphite target containing $\mathrm{Ni}$ or $\mathrm{Co}$ as catalyzer [363]. In a typical process, the synthesis is performed in a quartz tube, the graphite target placed at the center and all the system is heated in a tubular furnace at $\sim 1000^{\circ} \mathrm{C}$. The laser beam is focused onto the target which causes the evaporation of carbon and metallic catalyst in an inert gas flow. Vapors condensate on a cooled collector along the downstream producing CNTs with impurities [364]. This process is cost consumable since it uses high energy power to vaporize the target, but this process has a high yield and produces primarily SWCNTs. In [365] the production of CNTs by laser ablation is reviewed describing the 
growth mechanisms and options to make the synthesis more efficient. Other sources of information are [352,362].

Finally, another popular method for the synthesis of CNTs is the chemical vapor deposition (CVD). In a CVD process, hydrocarbon precursors as methane, acetylene, ethane, ethylene or alcohols ... [352,366], are decomposed at high temperature in presence of a metallic catalyst, e.g., $\mathrm{Ni}, \mathrm{Co}, \mathrm{Fe}[366]$. When the reaction gas passing through the flow furnace at high temperature about $1000^{\circ} \mathrm{C}$, hydrocarbon molecules decomposed into active carbon species on the catalyst surface and diffuse into the metal catalyst. Two different processes can then occur: in the tip-growth, the catalyst-substrate interaction is weak Figure 19A. The decomposed hydrocarbons diffuse down through the metal and the CNT grows between catalyst and substrate. In the opposite case, when the catalyst-substrate interaction is strong a base growth process take place. Again the decomposed hydrocarbons dissolve on the catalyst surface but now the precipitation is forced to emerge out from the metal's apex with $\mathrm{sp}^{2}$ structure. Both the processes end when the catalyzer particles are completely covered with excess of carbon.

\section{(A)}
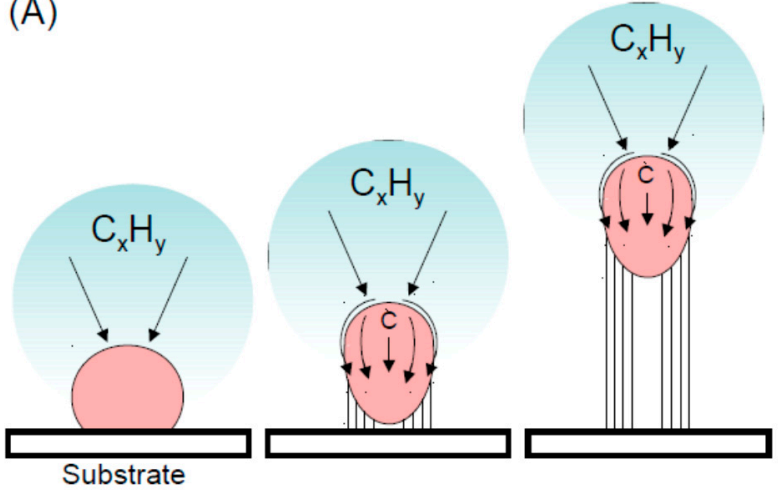

Growth stops

(B)
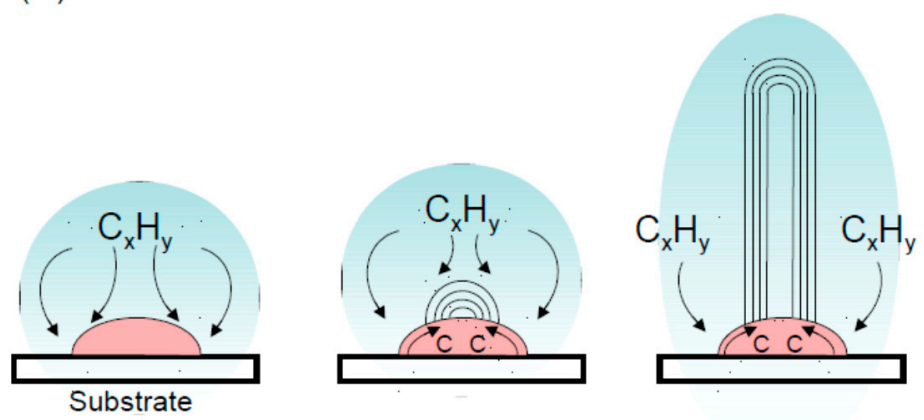

Figure 19. Widely-accepted growth mechanisms for CNTs: (A) tip-growth model, (B) base-growth model.

The characteristics of CNTs produced through CVD method depend on the hydrocarbon pressure and concentration, the nature of the catalyzer, the time of the reaction and temperature [362,366]. To lower the reaction temperature of the CVD process, plasmaenhanced CVD (PECVD) has been developed [367]. Besides lower temperature, advantages claimed include the ability to grow individual, free-standing, vertical SWCNTs, MWCNT and also carbon nanofibers on delicate substrates.

Lower cost and mass production can be obtained using the flame synthesis of CNTs. This method is capable of a controlled production of CNTs on the selected substrates by using the appropriate catalyst, the correct temperature and the adequate source of carbon. In the flame process, the catalyst is introduced in the flame where it condenses in spherical nanoparticles. $\mathrm{Ni}, \mathrm{Co}$, stainless steal and $\mathrm{Fe}$ are generally utilized as catalyst in the flame process [368]. As for the temperature, variation of the flame parameters/patterns 
strongly influences the final product. Several flame patterns, including premixed, partially premixed, and inverse diffusion flames, have been used for the production of CNTs and nanofibers [369]. Finally concerning the source of carbon, different fuels as methane, ethanol, ethylene, methylene acetylene, propane were investigated to check the effect on the production of SWCNT, MWCNT and their morphology. Detailed information can be found in [368]. In Figure 20 are reported TEM images of CNTs synthesized by different techniques.
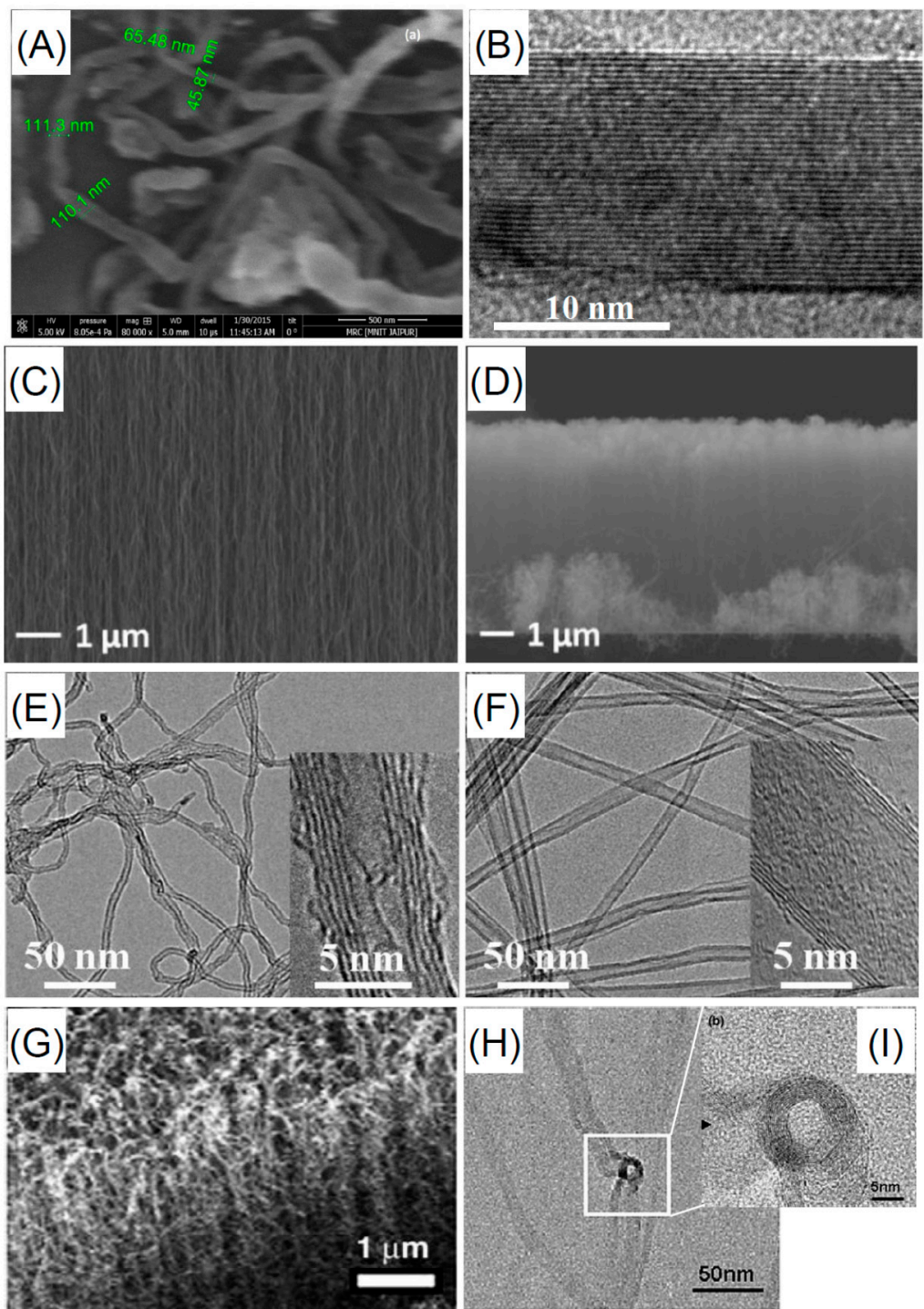

Figure 20. SEM (A) and HRTEM (B) images of MWCNTs synthesized by arc-discharge. Reproduced with permission from [370]. (C) Cross-sectional SEM images of vertically aligned CNTs grown on different buffer layers at $600{ }^{\circ} \mathrm{C}$ of $\mathrm{ALD} \mathrm{Al}_{2} \mathrm{O}_{3}$ and $(\mathbf{D})$ of $\mathrm{SiO}_{2}$ while $(\mathbf{E}, \mathbf{F})$ are the correspondent TEM images. Reproduced with permission from [371]. (G) A SEM image of CNT pillars on Si produced by pulsed lased deposition and (H) a TEM image of a junction connecting two CNTs and (I) its HRTEM magnification. Reprinted with permission from [372]. Copyright 2015 Taylor\&Francis, Copyright 2019 Springer, Copyright, Copyright 2005 Elsevier.

Most of the techniques used to synthesize CNTs, generate soot as a byproduct containing CNTs and other materials as amorphous carbon, fullerenes, nanocrystalline graphite, and metals introduced as catalysts for the synthesis. Freshly prepared CNTs need purification to separate them from the other form of carbon and from the metal catalysts. A variety of different purification methods have been developed for this purpose. However, common 
to all purification procedures are the following main steps: (i) removal of the large graphitic particles; filtration of CNTs and consequent resuspension in appropriate solvents; (ii) elimination of the metallic components via dissolution; (iii) and remove the amorphous carbon clusters and fullerenes, microfiltrations and chromatography for size selection [353]. To accomplish these tasks, chemical and physical purification processes can be used [373,374]. The chemical methods involve of concentrated acids or strong oxidants or treatments at high temperature generally in air or oxygen atmosphere. These treatments are used to accomplish a selective etching since they attack more easily amorphous carbon and carbon particles with respect to CNTs. However, both these treatments lead to consistent damage of the CNTs. Physical processes consist in filtration with membranes characterized by small pore size enabling separation of the CNTs from impurities and also to fractionating the CNTs by length. High-energy ultrasounds in the presence of the suitable solvents are used to get rid of the amorphous impurities [375]. Finally, centrifugation is applied to obtain the separation of particulate with different mass [376]. Low-speed centrifugation $(2000 \times g)$ is applied to remove amorphous carbon and leave SWCNTs and CNPs. High-speed centrifugation $(20,000 \times g)$ forces the CNPs to sediment while the SWCNTs remain suspended in aqueous media. Sedimentation and suspension depend not only on the mass but also on the different suspension stabilities e.g., surface charge of the particulate induced by functional groups [377].

\subsection{Carbon Nanotube Functionalization}

Since their discovery, several well consolidate routes to functionalize the CNTs were developed. Because of the graphitic nature, of the walls of pristine CNTs display a hydrophobic nature. As a consequence, van der Waals forces cause aggregation into bundles of freshly prepared CNTs. Therefore, it is difficult to separate CNTs and make a stable dispersion in water and in most solvents. This problem may be solved by functionalizing the CNT surface. Functionalization processes of CNTs were developed in the nineties and can be distinguished in covalent and non-covalent functionalization. Covalent functionalization consists in the formation of covalent bonds between a functional group and a carbon atom of the CNT. The covalent functionalization process involves several chemical reactions resulting in covalent chemical bonds between CNTs and the functional entities. They can be divided into two categories: (i) oxidation and end/defects functionalization, (ii) side wall covalent functionalization. An The defect-functionalization of CNTs proceeds by attacking the nanotubes wall defects by strong agents leaving holes functionalized with oxygenated functional groups [378]. Examples are depicted in Figure 21A,B.

(A)

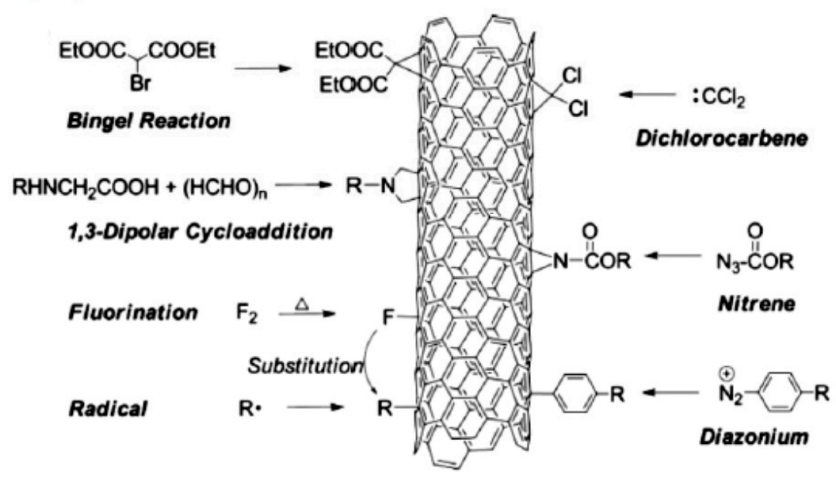

(B)

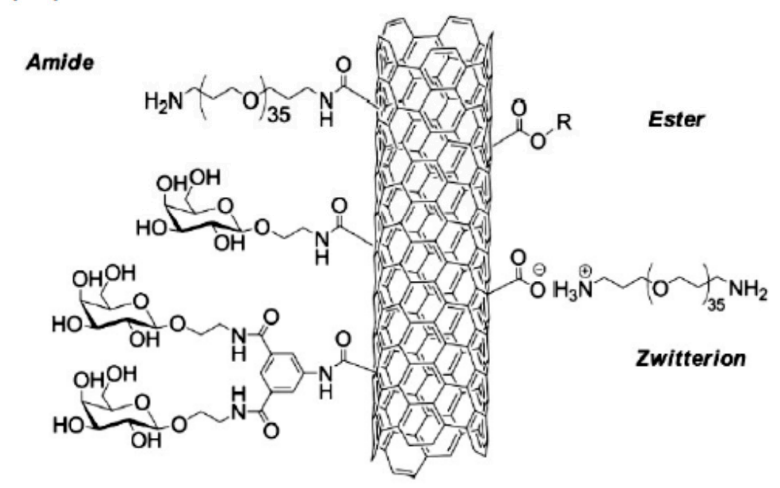

Figure 21. (A) Covalent addition reactions on the sidewall of carbon nanotubes. (B) Reactions targeting carboxylic acids (derived from nanotube surface defects). Adapted with permission from [379]. Copyright 2009 John Willey and Sons.

Generally, oxidation is performed using strong acids as $\mathrm{HNO}_{3}, \mathrm{H}_{2} \mathrm{SO}_{4}$, or their mixture [380] and increasing the reaction temperature [381], or strong oxidant as $\mathrm{KMnO}_{4}$ [382] 
or using ozone [383] or with reactive oxygen plasma [384]. These treatments induce the formation of acidic sites on the CNTs which are subsequently used for attaching other molecules. Oxidation of the CNTs generate oxygen containing functional groups such as carboxylic acid, ketone, alcohol and ester groups, that can be utilized to attach many other different types of chemical moieties [385]. These functional groups enable a rich chemistry allowing attachment of other molecules such as esterification [386], thiolation [387], silanation [388], polymer grafting [389], and some biomolecules [390] see Figure 21B.

In the direct covalent sidewall functionalization the formation of the covalent bond induces a change of the carbon hybridization from $\mathrm{sp}^{2}$ to $\mathrm{sp}^{3}$ and a simultaneous loss of p-conjugation typical of the aromatic rings of graphene. This process can be made by reaction with some molecules of a high chemical reactivity of atoms at the ends and at the defects of the CNTs. One of the main advantages of the CNTs functionalization is their dispersibility in solvents thanks to the polar or non-polar groups grafted on the surface. Examples of sidewall functionalization are depicted in Figure 22A,B.

(A)

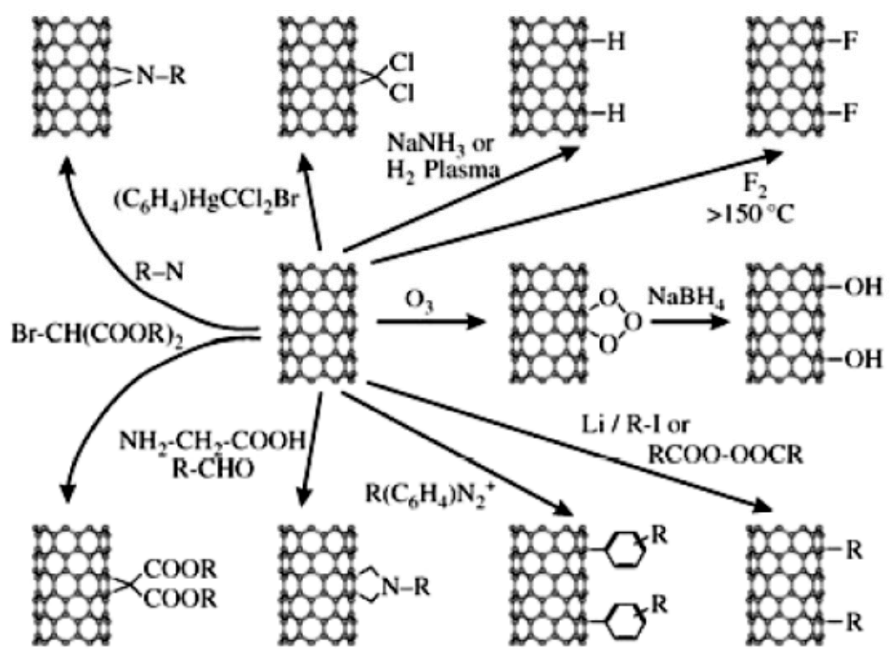

(B)

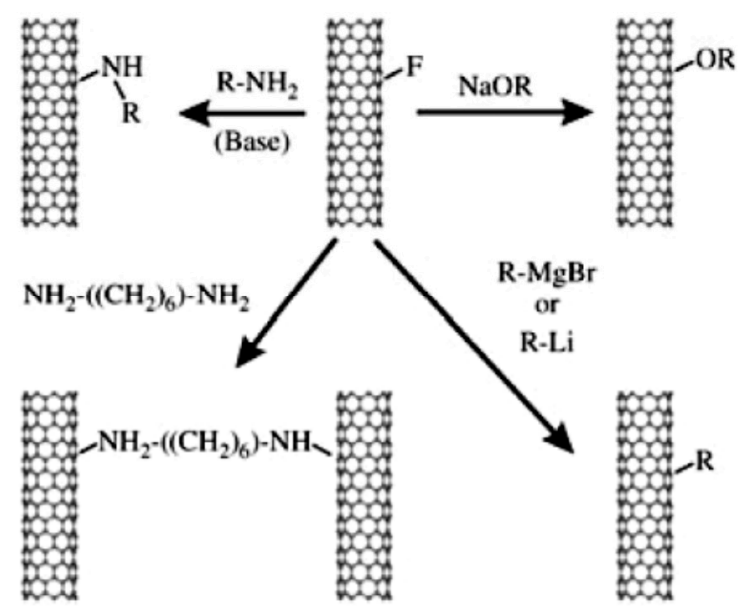

Figure 22. (A) Overview of possible addition reactions for the functionalization of the nanotube sidewall. (B) Functionalization of the sidewall through nucleophilic substitution reactions in fluorinated nanotubes. Reproduced with permission from [391]. Copyright 2005 John Wiley and Sons.

Fluorination of CNTs has become popular because providing substitution sites for additional functionalization [392] enabling replacements of the fluorine atoms by hydroxyl, amino, and alkyl groups [393]. Other possible chemical routes include the Diels-Alder cycloaddition [394], the carbene and nitrene addition [395], chlorination, bromination [396], azomethineylides [397], hydrogenation [398].

However, although covalent functionalization provides a rich list of possible routes to graft the desired molecules, there are some drawbacks. Functionalization accomplished though strong covalent bonds results in the rupture of the aromatic rings of the CNT graphitic lattice. This impacts on both the electrical and the mechanical properties of the CNTs. The disruption of the surface conjugated $\pi$ network introduces defects which can behave as rupture sites while each covalent bond act as a scatter point limiting the electrical conductivity [399]. Therefore, many efforts have been made to find convenient solutions to limit the CNT damage introduced by the functionalization. Preservation of CNT properties is achieved using the non-covalent functionalization. One of the main advantages of non-covalent functionalization is that it does not modify the carbon hybridization thus maintaining the hexagonal lattice with aromatic graphitic rings.

This preserves the structural and electrical properties of the CNTs because the non covalent functionalization is based on supramolecular complexation made by hydrogen 
bonds, adsorption based on van der Waals forces, by electrostatic forces or $\pi$-stacking interactions [400]. An example of $\pi$-stacking interactionsis shown in Figure 23 to attach bio-molecules to the CNT sidewalls.

$(\mathrm{A})$
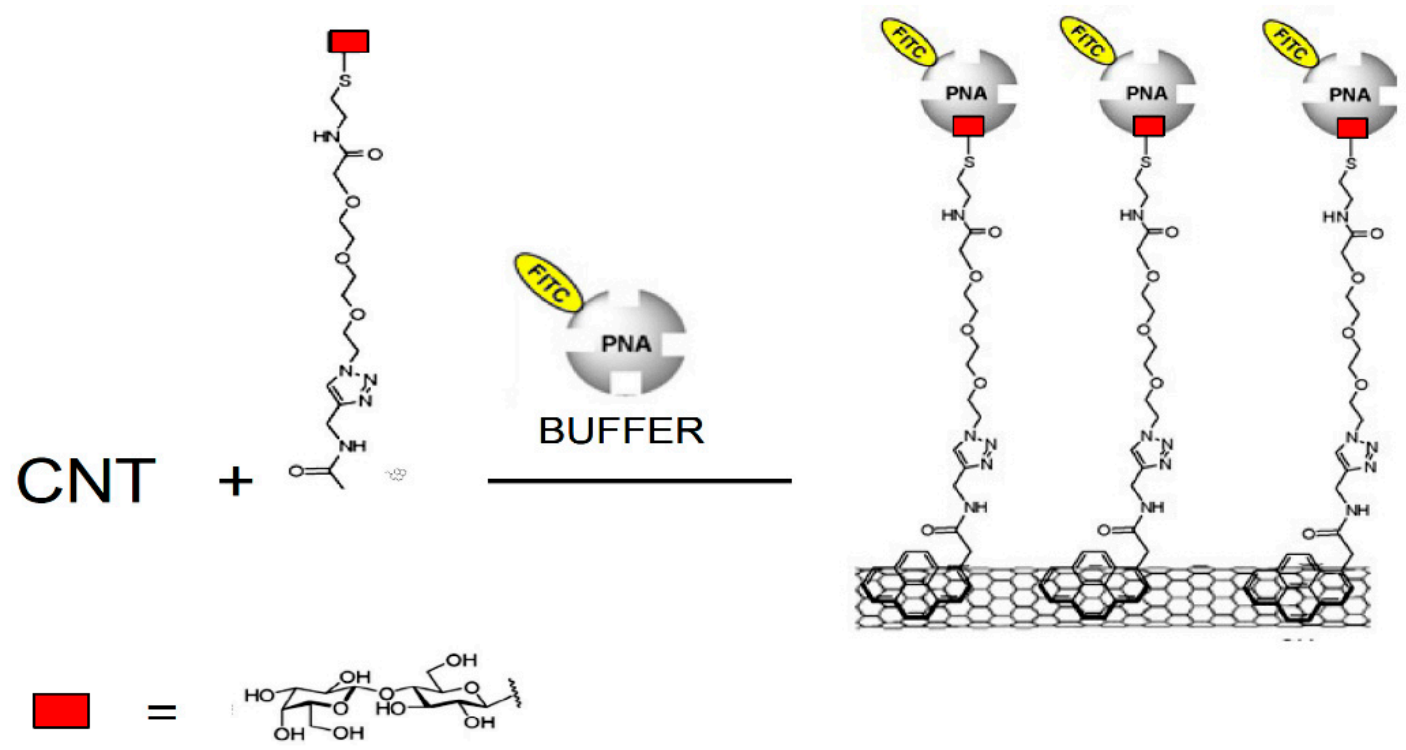

(B)

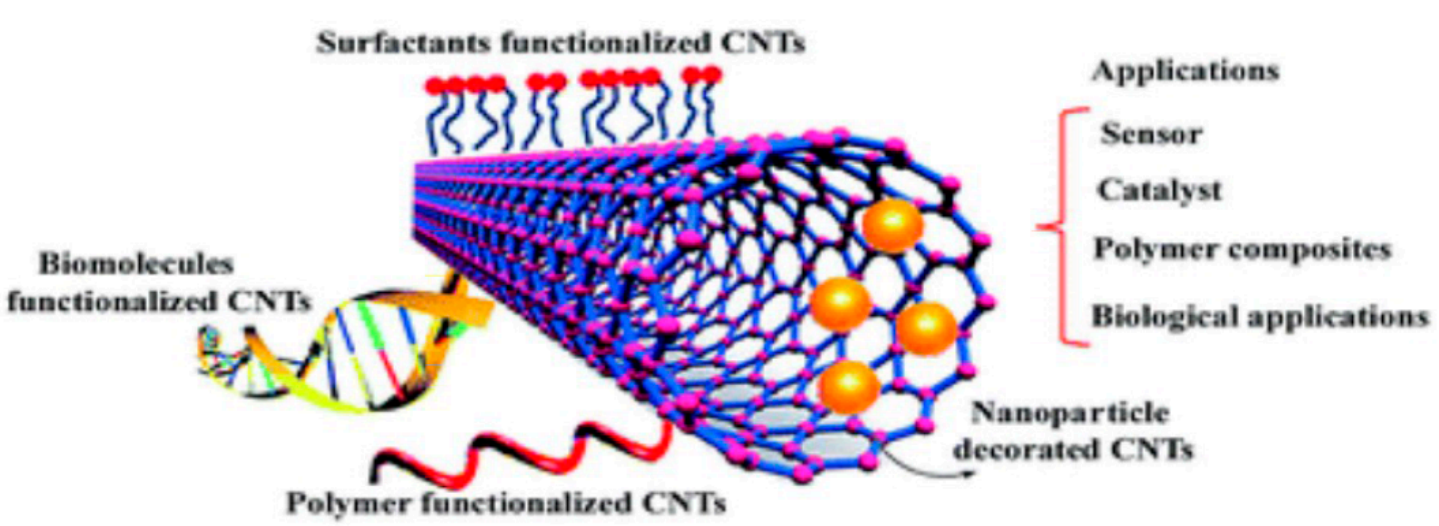

Figure 23. (A) $\pi-\pi$ interaction used to bind a lactose-specific receptor such as the Arachis hypogaea Peanut agglutinin (PNA) for selective recognition of lactose. Reproduced with permission from [401]. Copyright 2005 Royal Society of Chemistry; (B) adsorption functionalization of CNTs and applications. Reprinted with permission from [402]. Copyright 2009, 2016 Royal Society of Chemistry.

A side effect of the non-covalent functionalization is the lower stability caused by interactions between the grafted molecule and the CNTs which might be weak [403]. Adsorption of molecules including biomolecules is widely utilized to functionalize CNTs [400,402], some examples are depicted in Figure 23B. Non-covalent amino functionalized CNTs was obtained by adsorption of $\mathrm{H}_{2} \mathrm{~N}-\mathrm{CH}_{2} \mathrm{CH}_{2} \mathrm{O}-\mathrm{Na}$ to MWCNTs walls [404]. The functionalization preserves the electrical, the thermal and mechanical properties thus making the functionalized MWCNTs- $\mathrm{NH}_{2}$ promising filler for developing electrical conductive MWCNT/epoxy composites. Van der Waals forces are utilized to decorate CNTs with polymeric molecules. CNTs coated with polypyrrole, poly(methylene blue), poly-(neutral red), poly(acrylic acid) and poly(3-methylthiophene) were used to fabricate sensors for the detection of dopamine [405-409]. Phenols were detected using poly(urea-formaldehyde) functionalized CNTs [410]. The interaction by $\pi-\pi$ stacking is very versatile and the list of aromatic compounds that are employed is very rich [400,411,412]. Non-covalent 
functionalization of CNTs include also the adsorption of polymeric molecules [413], and biomolecules [414]. Some of the possible non-covalent functionalization processes of the CNTs are schematized in Figure 24. Functionalization of CNTs is covered in several review articles as [385,391,400,402,415].

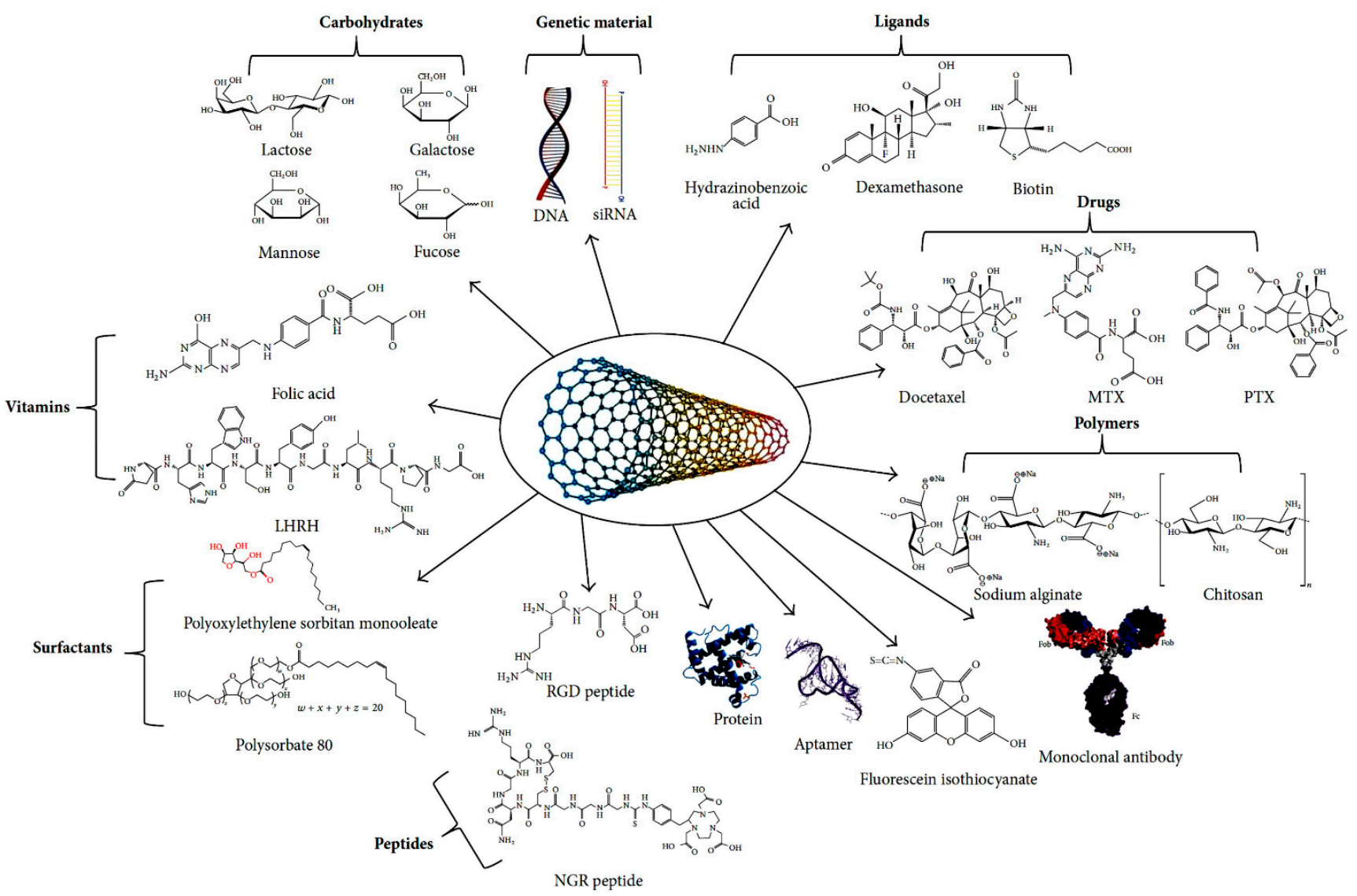

Figure 24. Non-covalent biofunctionalization of CNTs. Reprinted with permission from [414]. Copyright 2017 Hindawi.

\subsection{Carbon Nanotube Sensing}

The multiple and unique physical, mechanical and chemical properties of the CNTs make them suitable for a plethora of different applications. ranging from Their applications include but are not limited to electronics [416,417], energy, [418], environment [419], atomic force microscope [420] and nanomedicine [421], chemical and biochemical sensing [422-424]. Conducting and semiconducting properties of CNTs, the possibility to provide them with recognition elements and to organize them in nanowire networks make them the material of choice for sensing applications. In electrochemical CNT based sensors, sensing is based on charge transfer induced by the analyte that will change the conductivity of the CNTs modulating the concentration of the charge carriers. In normal conditions, CNTs are p-doped because of physisorption of oxygen molecules on their surfaces. As a consequence, Thus, further interaction with p-dopant molecules will increase hole concentration and increase the conductivity while while n-type dopants will increase the resistivity [425-427]. An excellent review regarding the use of the electronic properties of CNT for sensing application can be found in [428]. CNT conductivity may decrease also because of a reduction of the charge carrier mobility caused by charge carrier trapping or by scattering [429]. These mechanisms were utilized to detect $\mathrm{NO}_{2}$ via formation of nitro and nitrite groups [430].

A wide range of gases can be detected using CNT and some examples will be given hereafter. Ammonia vapors are toxic and can cause severe irritation of the respiratory sys- 
tem and pulmonary edema at high concentrations. Detection is based on the ability of $\mathrm{NH}_{3}$ molecules to adsorb on the CNTs walls without any need of particular functionalization changing their electric properties. The sensitivity obtained was as low as $20 \mathrm{ppb}$ with a LOD of $3 \mathrm{ppb}$ [431] and ascribable to the scrupulous preparation of the CNTs and of the sensing electrode. Other studies showed an enhancement of the sensor response to $\mathrm{NH}_{3}$ with the defect level [432]. Deposition of SWCNTs on different kinds of paper were used to fabricate $\mathrm{NH}_{3}$ sensors showing a large dynamic range from 0.5 to $5000 \mathrm{ppm}$ [433]. All the sensors showed a linear resistive response in the range 0.5 to $10 \mathrm{ppm}$ while the LOD varied upon the kind of paper used as a substrate form 0.36 to $2.7 \mathrm{ppm}$. In another work, poly(4-vinylpyridine)-CNTs functionalized with Pd nanoparticles showed an excellent sensitivity to thioethhers with a LOD of $0.1 \mathrm{ppm} \mathrm{[434].} \mathrm{NO}_{2}$ is another gas detected using a SWCNT dispersions onto interdigitated electrodes with a linear response in the range 6 and $100 \mathrm{ppm}$ and a LOD of $44 \mathrm{ppb}$ [435]. A higher sensitivity to $\mathrm{NO}_{2}$ was obtained in using a PECVD reactor to deposit a thin film of MWCNTs on Pt electrodes with a dynamic response from 10 to $100 \mathrm{ppb}$ and a LOD of $10 \mathrm{ppb}$ [436]. $\mathrm{NH}_{3}$ and $\mathrm{NO}_{2}$ sensors were also fabricated coupling CNTs with metal oxides [437,438]. As an exaple $\mathrm{SnO}_{2}$ was coupled to SWCNTs to detect $\mathrm{NH}_{3}$ reaching a LOD of $1 \mathrm{ppm}$ for $\mathrm{NH}_{3}$ and of $20 \mathrm{ppb}$ for $\mathrm{O}_{3}$ [439]. Other metals used to enhance the sensitivity of the CNT electrodes to $\mathrm{CO}, \mathrm{CO}_{2}, \mathrm{NH}_{3}, \mathrm{CH}_{4}$ and $\mathrm{NO}_{2}$ are $\mathrm{Co}, \mathrm{Cu}, \mathrm{Pb}, \mathrm{Pd}, \mathrm{Ni}, \mathrm{Pt}, \mathrm{Ru}, \mathrm{Ag}[440,441]$. Metals are also used to detect $\mathrm{H}_{2}$ and $\mathrm{CH}_{4}$. Pt [442] and $\mathrm{Pd}$ [443] metals are used to decorate HGCNTs for the detection of $\mathrm{H}_{2}$. $\mathrm{Pd}$ decorated SWCNTs are also used to detect $\mathrm{CH}_{4}$ with a dynamic range of 6-100 ppm [444]. $\mathrm{CH}_{4}$ sensors were fabricated also using semiconducting metal oxides as $\mathrm{SnO}_{2}$ or $\mathrm{ZnO}$ functionalized CNTs [445,446]. In the first case a sensitivity of $10 \mathrm{ppm}$ was obtained while in the second the LOD was $2 \mathrm{ppm}$. Electrochemical sensing was also applpied to detect CO although weak interaction and the limited charge transfer with CNTs [447]. Fe-porphyrin functionalized SWCNT were used to detect CO reaching a LOD of $80 \mathrm{ppm}$. Unfortunately, $\mathrm{CO}_{2}$ does not engage in strong binding or charge transfer. Boron and nitrogen doping have been observed theoretically to increase the interaction between $\mathrm{CNTs}$ and $\mathrm{CO}, \mathrm{CO}_{2}$ thus increasing the sensitivity [448]. Oxygen plasma was another strategy to increase the $\mathrm{CO}$ detection sensitivity reaching a LOD of $5 \mathrm{ppm}$ [449]. The sensor showed good selectivity towards other gaseous species as $\mathrm{H}_{2}$ and $\mathrm{CH}_{4}$. $\mathrm{CO}$ can be detected also using an organoCo-CNT complex via chemoresistive measurements [450]. The CNTs were functionalized with an organo- $\left(\mathrm{CP}^{\wedge} \mathrm{CoI}_{2}\right)$ complex which is able to coordinate $\mathrm{CO}$ molecules as depicted in Figure 25.

The sensor showed a good gas selectivity towards other gasses as $\mathrm{CO}_{2}, \mathrm{CH}_{4}, \mathrm{C}_{2} \mathrm{H}_{2}$, $\mathrm{H}_{2}$, air, and a LOD of $90 \mathrm{ppm}$. Also for the detection of $\mathrm{CO}_{2}$ metals can improve the sensor performances. Among others, $\mathrm{Au}$ [451], Pd (theoretically) [452], $\mathrm{SnO}_{2}$ [453]. In the first case a plasmonic structure was performed depositing Au-docorated CNTs on a Bragg grating produced on a single mode optical fiber. The sensor showed a decent selectivity with respect to $\mathrm{CH}_{4}, \mathrm{C}_{2} \mathrm{H}_{6}, \mathrm{C}_{3} \mathrm{H}_{8}, \mathrm{C}_{4} \mathrm{H}_{10}$ air with a LOD of $150 \mathrm{ppm}$. Both sulphur based gases such as $\mathrm{H}_{2} \mathrm{~S}$ or $\mathrm{SO}_{2}$ can also be detected using $\mathrm{Au}$ and $\mathrm{Pt}$ decorated CNTs. $\mathrm{H}_{2} \mathrm{~S}$ is an electron donor while $\mathrm{SO}_{2}$ is an electron acceptor. Au decorated CNTs were able to detect $\mathrm{H}_{2} \mathrm{~S}$ wit a sensitivity of $3 \mathrm{ppb}$ [454]. In the case of $\mathrm{SnO}_{2}$ decorated CNT the chemo-resistive device had a sensitivity to $\mathrm{CO}$ of $1 \mathrm{ppm}$. There are just a few examples of $\mathrm{SO}_{2}$ which was detected using SWCNT at room temperature [455] with a sensitivity 200 times higher than that for $\mathrm{O}_{2}$. Finally also benzene, toluene, and xylene can be detected using CNT based sensors [456] with a LOD between 500 ppb-10 ppm. Detection of volatile organic compounds (VOCs) plays an increasing role in the environmental control and in biomedical applications. Benzene, toluene, methanol, ethanol and acetone were detected by impedence spectroscopy with a LOD of $1.62 \mathrm{ppm}$ and $1.8 \mathrm{ppm}$ for benzene and toluene respectively [457]. MWCNTs treated in an oxygen plasma to increase the sensitivity to benzene vapors to $800 \mathrm{ppb}$. VOCs detection is based on the adsorption of the organic molecules on the CNT walls which depending on the VOC chemistry, might be a rather slow process. To enhance the sensor performances in detecting VOCs as acetone CNTs 
were functionalized with poly-porphyrins rendering the sensor dynamic range very large from 50 to 230,000 ppm with a LOD of $9 \mathrm{ppm}$ and a stable response over a period of 180 days [458]. Another possibility is the decoration of amine functionalized CNTs with Au nanoparticles which improves the detection of hexane, toluene, trichloroethylene and chloroform against polar compounds such as water, proponol and ethanol [459].

(A)

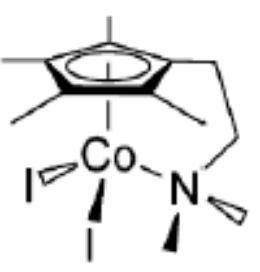

$\left[\mathrm{Cp}^{\wedge} \mathrm{Col}_{2}\right]$
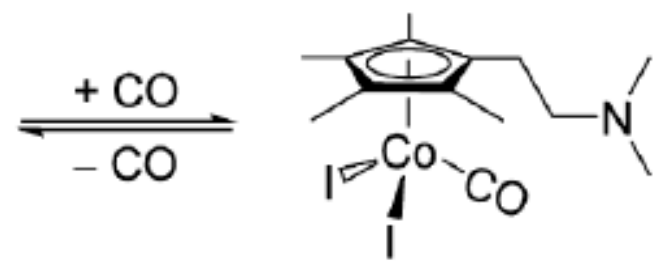

$\left[\mathrm{Cp}^{\wedge} \mathrm{Col}_{2}(\mathrm{CO})\right]$
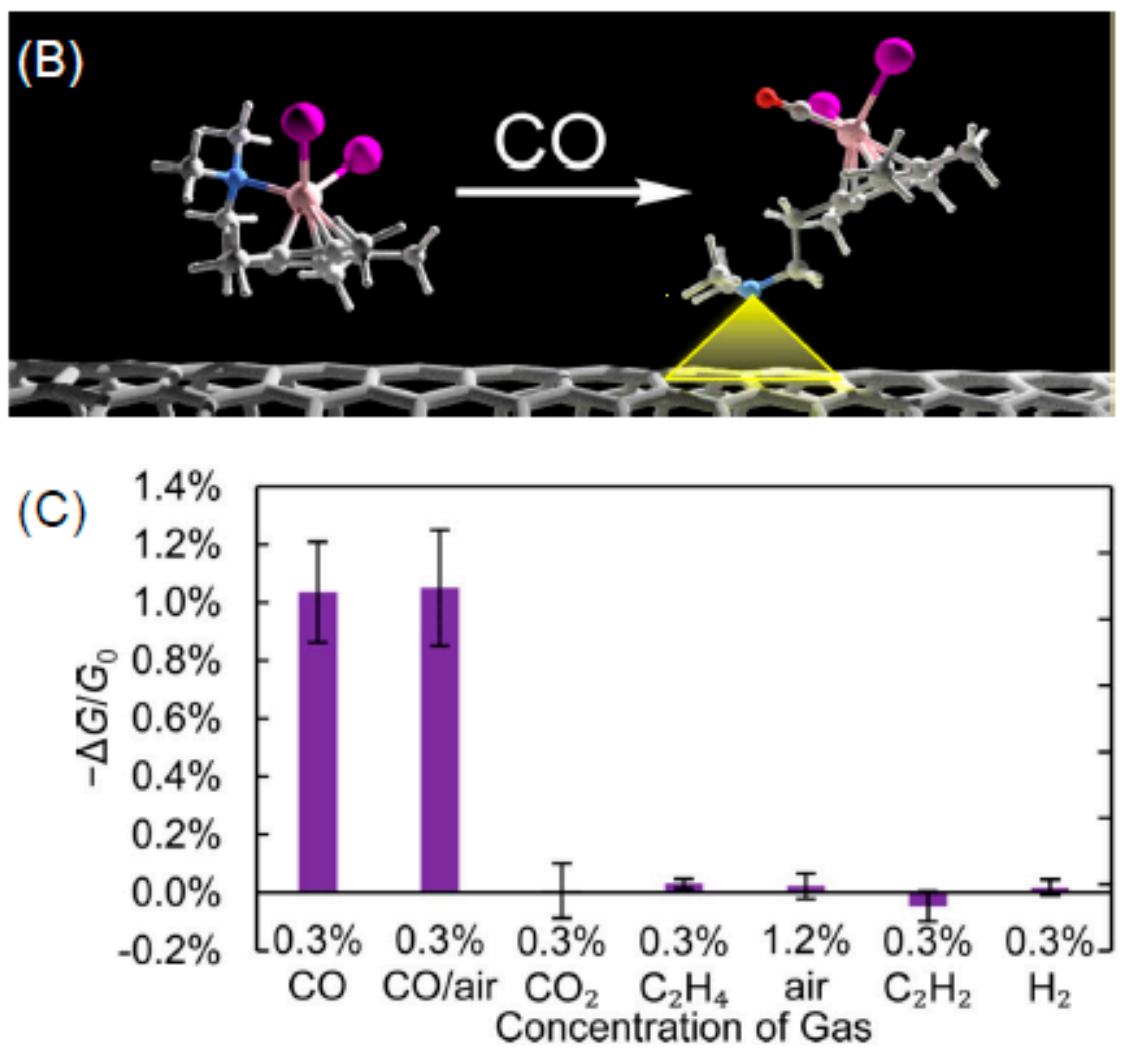

Figure 25. (A,B) reversible $\mathrm{CO}$ Binding to $\left[\mathrm{Cp}^{\wedge} \mathrm{CoI}_{2}\right]$. (C) Average conductance changes of four chemiresistors in response to $60 \mathrm{~s}$ exposures to various gases diluted in $\mathrm{N}_{2}$ unless otherwise indicated. Error bars represent 1 standard deviation across the four devices. Reprinted with permission from [450]. Copyright 2016 American Chemical Society.

Also changes of $\mathrm{pH}$ influence the electrical properties of CNTs allowing detection. Poly(aniline) SWCNTs are sensitive to protonation rendering the complex useful for $\mathrm{pH}$ sensing [460]. High stability was obtained by coordinating SWCNTs with poly(1-amino anthracene) resulting in a sensor providing a stable response over a period of 120 hours in a 2-12 $\mathrm{pH}$ range [461]. Other information can be found in [462-464]. The possibility to integrate CNTs in smart low cost microcircuits enables their use as sensors in the agro-food sector. An example is the control of fruit ripeness through the control of the fruit-ripening hormone ethylene. Virgin CNTs are insensitive to ethylene molecules which then can be detected only by an appropriate functionalization. It has been demonstrated the decoration of CNTs with $\mathrm{SnO}_{2}$ provides the required sensitivity with a LOD of 3 ppm [465]. However, 
$\mathrm{SnO}_{2}$ is also sensitive to $\mathrm{NO}_{2}$ which may be a problem which was solved by a complexation of SWCNTs with fluorinated tris(pyrazolyl)-borate copper(I) [466]. CNTs are also utilized to detect food smells $[467,468]$ using a OR 2AG1 protein/CNTs complex and reaching a sensitivity of $1 \mathrm{fM}$ for Amylbutirate. Food integrity was controlled through detection of bio-amines and ammonia using cobalt meso-aryl-porphyrin complexes [469]. Preservation of food and product integrity takes advantage form the control of the presence of oxygen in the packaging. As already described CNTs can be utilized to detect oxygen and thus the degree of product quality [470].

An important application of CNT is the detection of biomolecules which can be important for biomedical applications. Diabete is one of the pathologies experiencing an increasing impact in modern societies. As a matter of fact, detection of glucose is one of the most frequently performed medical tests. The sensing is based on the catalytic effect of glucose oxidase (GOx)/CNT complex and a direct electron transfer to the sensor upon glucose recognition [471]. However this electrode suffers from poor stability, problem which was solved adsorbing GOx onto Pt nanoparticle decorated CNT electrodes. The new sensor displayed a good linear range $(0.1-13.5 \mathrm{mM})$ and high sensitivity of $14 \mu \mathrm{A} / \mathrm{mM}$ [472]. Other authors utilized a forest of vertically aligned CNTs coordinated with the glucose oxidase enzyme and were able to demonstate that $\mathrm{CNT}$ act as conductive nanowires connecting the enzyme redox-active site to the transducer surface [473]. Other authors developed a Polyaniline/functionalized SWCNT/Prussian Blue complex able to selectively detect glucose among acetaminophen, uric acid, lactate, and ascorbic acid which are common interfering species [474] with a sensitivity of $18.6 \mu \mathrm{A} /\left(\mathrm{m} \mathrm{cm}^{2}\right)$. In another work, CNTs served as chemoresisitive glucose sensors [475]. To render highly hydrophilic, SWCNTs were functionalized with poly(4-vinylpyridine) chains and with GOx molecules. Exposure to glucose induce the formation of hydrogen peroxide which causes a decrease if the SWCNTs resistance. This last changed linearly in a range between 0.08 and $2.2 \mathrm{mM}$ glucose. In addition the hydrogen peroxide attacks the SWCNT walls increasing the number of defects thus inducing changes in the $\mathrm{I}(\mathrm{D}) / \mathrm{I}(\mathrm{G})$ Raman components allowing an indirect measure of the glucose concentration.

Besides glucose also the measurement of the cholesterol level in the blood stream is important. This were accomplished using screen printed electrodes functionalized with cholesterol esterase, peroxidase, oxidase and MWNTs [476]. The sensor showed a linear response over a range from 20 to $200 \mathrm{mg} / \mathrm{dL}$ and 100 to $400 \mathrm{mg} / \mathrm{dL}$ for uric acid and cholesterol and the respective sensitivities were of 0.0721 and $0.0059 \mu \mathrm{A}$ per $\mathrm{mg} / \mathrm{dL}$. In another work authors used CVD grown vertically aligned CNTs which were subsequently functionalized with polyaniline and cholesterol esterase to detect cholesterol [477]. The sensor showed a quasi-linear response in the range $50-300 \mathrm{mg} / \mathrm{dl}$ with a sensitivity of $22 \mu \mathrm{A} /(\mathrm{mg} \mathrm{dL})$. The sensor showed good repeatability and selectivity toward interfering glucose, uric acid acetaminophen and ascorbic acid. In parallel to the detection of glucose, it has been developed a CNT based sensor for insulin enzyme which is important for the treatment of type I diabetes [478]. The sensor was fabricated using dimethylformamide modified CNTs casted on a glassy carbon electrode. The sensor at physiological $\mathrm{pH}$ showed a linear response up to $1000 \mathrm{nM}$ insulin and a LOD of $14 \mathrm{nM}$. In [479] RuOx modified CNTs were utilized to sense insulin. The modified electrode showed a linear response in the range $10-800 \mathrm{nM}$ and a LOD of $1 \mathrm{nM}$. Another important parameter is the concentration of $\mathrm{NO}$ in the blood. Nitric oxide (NO) has different functions acting as a neurotransmitter, is involved in regulation of feelings, of pain, appetite, the circadian cycle, thermoregulation, synaptic plasticity, and neural secretion [480]. SWCNT are known to emit in the NIR region but the fluorescence signal is quenched in presence of NO thus enabling an optical detection. In [481] CNTs were modified binding DNA sequence of d(AT) ${ }_{15}$ oligonucleotides thus enabling single molecule sensitivity. In addition fluorescence quenching was shown to be proportional to the NO concentration adsorbed on the electrode. In another work, DNA oligonucleotide ds(AAAT) ${ }_{7}$ were bonded to CNTs using polyethylene glycol spacers [482]. These modified CNTs were injected in mice and allowed in vivo measurements of NO 
with a LOD of $1 \mu \mathrm{M}$. CNT functionalized with N-hydroxyphenyl maleimide were also used to sense other biomolecules as epinephrine [483] with a linear response in the range $0.09-5.90 \mathrm{ng} / \mathrm{mL}$ and a LOD $=0.02 \mathrm{ng} / \mathrm{mL}$. Au decorated CNTs were utilized to sense dopamine [484] via square wave voltammetry with a linear range between $0.48 \mu \mathrm{M}$ to $5.7 \mu \mathrm{M}$, a LOD of $0.071 \mu \mathrm{M}$. More information may be found in [485]. SWCNTs were used to detect $\mathrm{H}_{2} \mathrm{O}_{2}$ in carcinoma cells [486] via fluorescence quenching. Sensitivity of the technique is down to the single $\mathrm{H}_{2} \mathrm{O}_{2}$ molecule secreted by A431 carcinoma cells in in vitro experiments.

Besides biomolecule sensors, CNTs are also used to fabricate DNA sensors. Ability of detecting specific DNA sequences is important for the diagnosis and the treatment of diseases generated by DNA aberration and genetic disorder. A DNA sensor was fabricated with mercaptohexonal and thiolated DNA sequence were attached to gold electrodes [487]. Hybridization with the complementary DNA sequence occurring on the sidewalls of SWCNT-FET induced a pronounced electrical conductance change due to the modulation of energy level alignment between SWNT and gold contact [487]. The sensing mechanism is depicted in Figure 26A.

(A)

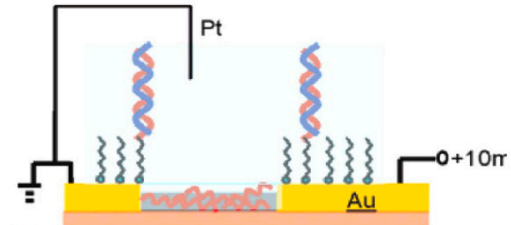

Thiolated DNA (Probe)

3 Complementary Target DNA (CM)

MercaptoHexanol $(\mathrm{MCH})$
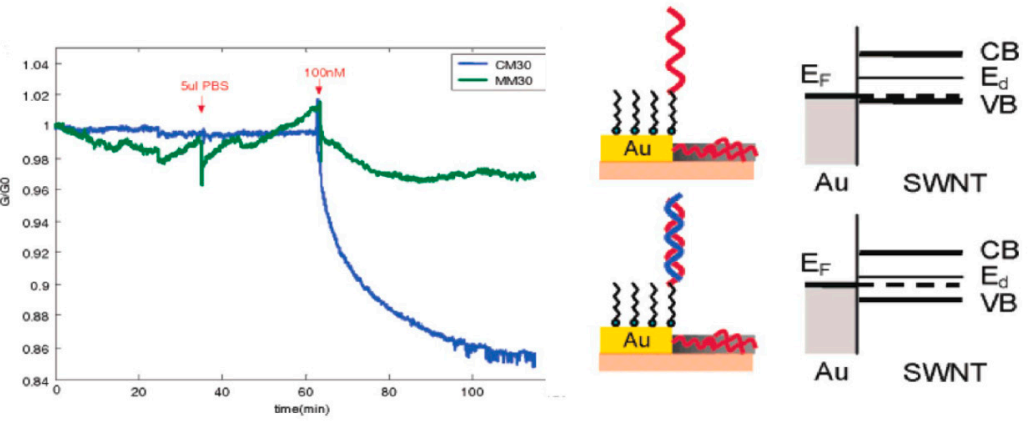

(B)

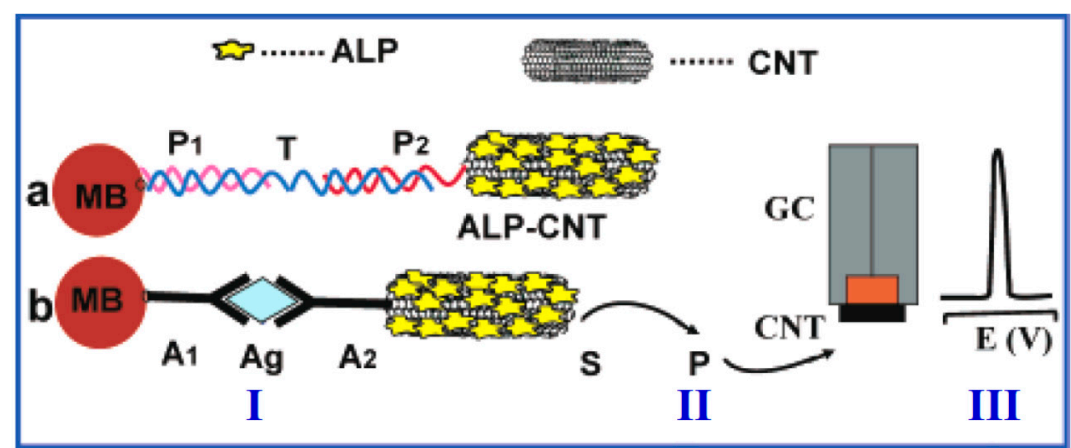

Figure 26. Detection modalities in CNT based sensors. (A) Left: schematic illustration of a single device during electrical measurement. Complementary ssDNA oligos hybridize to thiolated ssDNA coimmobilized with $\mathrm{MCH}$ on the gold electrodes. Center: the electrical conductance of a SWNT functionalized ssDNA capturing exhibits almost no change upon mismatch while shows a marked decrease when hybridized with the complementary target DNA. Right: schematic illustration of energy level alignment before and after DNA hybridization. Reprinted with permission from [487]. Copyright 2006 American Chemical Society (B) Schematic representation of the analytical protocol: (I) Capture of the ALP-loaded CNT tags to the streptavidin-modified magnetic beads (a) by a sandwich DNA hybridization or (b) Ab-Ag-Ab interaction. (II) Enzymatic reaction. (III) Electrochemical detection of the product of the enzymatic reaction at the CNT-modified glassy carbon electrode. Reprinted with permission from [488]. Copyright 2004 American Chemical Society.

The sensitivity of the sensor was $4 \times 10^{13}$ molecules $/ \mathrm{cm}^{2}$. Frequently, in a sensor an electrode is modified with CNTs functionalized with DNA sequences. The DNA hybridization with the complementary sequence leads to a change of the current flowing through the electrode [489]. To increase the detection sensitivity, authors used alkaline 
phosphatase enzyme to preconcentrate the analyte thus amplifying the electrical sensing of proteins and DNA resulting in a LOD of the target DNA of $1 \mathrm{fg} \mathrm{mL}^{-1}$ [488]. Schematic representation of the analytical protocol and detection is represented in Figure 26B. It is also possible to fabricate CNT based devices for sensing any kind of DNA sequence [490]. To achieve this result, authors functionalized CNTs with a specific peptide (with the sequence Fmoc-RRMEHRMEW) as natural molecules having the unique ability to selectively bind to universal DNA sequences. The sensor showed a broad sensing range from nearly $1.6 \times 10^{-4}$ to $5 \mu \mathrm{mol} \mathrm{L}{ }^{-1}$ and a much lower detection limit of approximately $0.88 \mu \mathrm{g} / \mathrm{L}$. An easy detection of DNA sequences was performed by mixing oxygen-functionalized MWCNTs in a buffer solution containing the DNA sequences [491]. The MWCNTs were able to modulate the sensor electrochemical current depending on the sequence of the target DNA and exhibited a LOD of $141.2 \mathrm{pM}$ and a good DNA sequence differentiation. There are other sectors in which CNT based sensors are applied: detection of dangerous metal ions [492,493]. $\mathrm{Pb}, \mathrm{Cd}, \mathrm{Hg}$, As, $\mathrm{Zn}, \mathrm{Cu}, \mathrm{Ag}, \mathrm{Cr}, \mathrm{Ni}$, and $\mathrm{Ti}$, ecc may be detected using $\mathrm{CNT}$ based sensors [494]. For $\mathrm{Pb}, \mathrm{Cd}, \mathrm{Cu}, \mathrm{Zn}$ the LOD varies between 0.017 to $4.4 \mathrm{ppb}$ [494]. CNT based sensors are utilized to detect pesticides and an overview is presented in [495]. Also pathogens may be sensed as presented in [496] or explosives [497].

Table 5 summarizes characteristics of CNT based sensors.

Table 5. Detection modality, analyte and detection limit of CNT based sensors.

\begin{tabular}{|c|c|c|c|c|}
\hline Method & Functionalization & Analyte & Performances & Reference \\
\hline Chemo-Resistivity & $\begin{array}{c}\text { GOx-poly(4- } \\
\text { vinylpyridine)/SWCNT }\end{array}$ & Glucose & $\begin{array}{c}\text { Linear between } 0.08 \\
\text { and } 2.2 \mathrm{mM}\end{array}$ & [475] \\
\hline Chemo-Resistivity & - & $\mathrm{NH}_{3}$ & $20 \mathrm{ppb}$ & [431] \\
\hline Chemo-Resistivity & Paper/CNTs & $\mathrm{NH}_{3}$ & 0.36 to $2.7 \mathrm{ppm}$ & [433] \\
\hline Chemo-Resistivity & $\begin{array}{c}\text { Pd/poly(4-vinylpyridine)- } \\
\text { CNTs }\end{array}$ & Thioethers & $0.1 \mathrm{ppm}$ & [434] \\
\hline Conductivity & Pristine SWCNT & $\mathrm{NO}_{2}$, nitrotoluene & $44 \mathrm{ppb}, 262 \mathrm{ppb}$ & [435] \\
\hline Chemo-Resistivity & Pt/MWCNTs films & $\mathrm{NO}_{2}$ & $10 \mathrm{ppb}$ & [436] \\
\hline Chemo-Resistivity & $\mathrm{SnO}_{2} / \mathrm{SWCNTs}$ & $\mathrm{NH}_{3}, \mathrm{O}_{3}$ & $1 \mathrm{ppm}, 20 \mathrm{ppb}$ & [439] \\
\hline Chemo-Resistivity & $\mathrm{SnO}_{2} / \mathrm{MWCNTs}$ & $\mathrm{CH}_{4}$ & 10 ppm & [445] \\
\hline Chemo-Resistivity & $\mathrm{ZnO} / \mathrm{MWCNTs}$ & $\mathrm{CH}_{4}$ & $2 \mathrm{ppm}$ & [446] \\
\hline Amperometric & Fe Porphyrin/SWCNTs & $\mathrm{CO}$ & 80 ppm & [447] \\
\hline Amperometric & Oxidized CNTs & $\mathrm{CO}$ & 5 ppm & [449] \\
\hline Chemo-Resistivity & $\mathrm{CP}^{\wedge} \mathrm{CoI}_{2} / \mathrm{CNTs}$ & $\mathrm{CO}$ & 90 ppm & [450] \\
\hline Plasmonics & $\mathrm{Au} / \mathrm{CNTs}$ & $\mathrm{CO}_{2}$ & 150 ppm & [451] \\
\hline Chemo-Resistivity & $\mathrm{SnO}_{2} / \mathrm{CNTs}$ & $\mathrm{CO}$ & $1 \mathrm{ppm}$ & [453] \\
\hline Amperometric & Fe-Porphyrine/CNTs & Benzene, xylene, toluene & 500 ppb-10 ppm & [456] \\
\hline $\begin{array}{l}\text { Impedence } \\
\text { spectroscopy }\end{array}$ & Oxygen functionalities & $\begin{array}{c}\text { Benzene, toluene, } \\
\text { methanol, ethanol and } \\
\text { acetone }\end{array}$ & $\begin{array}{l}1.62 \text { for benzene } \\
1.8 \mathrm{ppm} \text { for toluene }\end{array}$ & [457] \\
\hline Chemo-Resistivity & Poly-porphyrins/CNTs & Acetone & 9 ppm & [458] \\
\hline Chemo-Resistivity & Poly(aniline)/SWCNTs & $\mathrm{HCl}, \mathrm{NH}_{3}, \mathrm{pH}$ & $100 \mathrm{ppb}$ for $\mathrm{HCl}$ & [460] \\
\hline
\end{tabular}


Table 5. Cont

\begin{tabular}{|c|c|c|c|c|}
\hline Method & Functionalization & Analyte & Performances & Reference \\
\hline Chemo-Resistivity & $\begin{array}{c}\text { poly(1-amino } \\
\text { anthracene)/SWCNTs }\end{array}$ & $\mathrm{pH}$ & - & [461] \\
\hline Chemo-Resistivity & $\mathrm{SnO}_{2} / \mathrm{CNTs}$ & Ethylene, $\mathrm{NO}_{2}$ & $\begin{array}{l}3 \text { ppm for ethylene } \\
50 \mathrm{ppb} \text { for } \mathrm{NO}_{2}\end{array}$ & [465] \\
\hline Chemo-Resistivity & $\begin{array}{l}\text { tris(pyrazolyl)-borate } \\
\text { copper(I)/CNTs }\end{array}$ & Ethylene & $<1$ ppm & [466] \\
\hline Chemo-Resistivity & OR 2AG1 protein/CNTs & Amyl Butirate & $1 \mathrm{fM}$ & [467] \\
\hline Chemo-Resistivity & $\begin{array}{l}\text { cobalt meso-aryl- } \\
\text { porphyrin/CNT }\end{array}$ & $\begin{array}{l}\mathrm{NH}_{3}, \text { putresceine, } \\
\text { cadaverine }\end{array}$ & $0.5 \mathrm{ppm}$ for $\mathrm{NH}_{3}$ & [469] \\
\hline Cyclic voltammetry & $\mathrm{GOx} / \mathrm{CNT}$ & Glucose & $0.5 \mathrm{mM} / \mathrm{L}$ & [471] \\
\hline Amperometry & $\mathrm{Pt} / \mathrm{CNT}$ & Glucose & $14 \mu \mathrm{A} / \mathrm{mM}$ & [472] \\
\hline Amperometry & $\mathrm{GOx} / \mathrm{CNT}$ & Glucose & $\begin{array}{l}\text { sensitivity of } 18.6 \\
\qquad \mu \mathrm{A} /\left(\mathrm{m} \mathrm{cm}^{2}\right)\end{array}$ & {$[474]$} \\
\hline Chemo-Resistivity & $\mathrm{GOx} / \mathrm{CNT}$ & Glucose & $2.2 \mathrm{mM}$ & [475] \\
\hline Amperometry & $\begin{array}{c}\text { Uricase/CNTs, } \\
\text { cholesterol-esterase/CNTs }\end{array}$ & Cholesterol, uric acid & $\begin{array}{c}0.0721 \text { and } 0.0059 \mu \mathrm{A} \\
\text { per } \mathrm{mg} / \mathrm{dL}\end{array}$ & [476] \\
\hline Amperometry & $\begin{array}{c}\text { Polyaniline and cholesterol } \\
\text { esterase/CNTs }\end{array}$ & $\begin{array}{l}\text { Cholesterol, glucose, uric } \\
\text { acid acetaminophen and } \\
\text { ascorbic acid }\end{array}$ & $22 \mu \mathrm{A} /(\mathrm{mg} \mathrm{dL})$ & [477] \\
\hline Amperometry & $\mathrm{DMF} / \mathrm{CNTs}$ & Insulin & $14 \mathrm{nM}$ & [478] \\
\hline Amperometry & $\mathrm{RuOx} / \mathrm{CNTs}$ & Insulin & $1 \mathrm{Nm}$ & [479] \\
\hline Fluorescence turn-off & SWCNT & NO & Single molecule & [481] \\
\hline Fluorescence turn-off & $\begin{array}{c}\mathrm{ds}(\mathrm{AAAT})_{7} \\
\text { oligonucleotide/CNTs }\end{array}$ & $\mathrm{NO}$ & $1 \mu \mathrm{M}$ in vivo & [482] \\
\hline Amperometry & $\begin{array}{l}\text { N-hydroxyphenyl } \\
\text { maleimide/CNTs }\end{array}$ & Epinephrine & $0.02 \mathrm{ng} / \mathrm{mL}$ & [483] \\
\hline $\begin{array}{l}\text { Square wave } \\
\text { voltammetry }\end{array}$ & $\mathrm{Au} / \mathrm{CNTs}$ & Dopamine & $0.071 \mu \mathrm{M}$ & [484] \\
\hline Fluorescence turn-off & SWCNTs & $\mathrm{H}_{2} \mathrm{O}_{2}$ & Single molecule & [486] \\
\hline Resistivity & SWCNTs & DNA & $\begin{array}{l}4 \times 10^{13} \\
\text { molecules } / \mathrm{cm}^{2}\end{array}$ & [487] \\
\hline Chronopoterntiometric & $\begin{array}{c}\text { alkaline } \\
\text { phosphatase/CNTs }\end{array}$ & DNA & $1 \mathrm{fM}$ & [488] \\
\hline Amperometry & $\begin{array}{c}\text { Fmoc- } \\
\text { RRMEHRMEW/CNTs }\end{array}$ & DNA & $0.88 \mu \mathrm{g} / \mathrm{L}$ & [490] \\
\hline Amperometry & Oxygen functionalities & DNA & $141.2 \mathrm{pM}$ & [491] \\
\hline
\end{tabular}

As a conclusion of this brief review on the use of CNTs for sensing, it clearly appears the versatility of these carbon nanostructures whose prominent properties enable a variety of different sensing applications. CNTs appear as the material of choice for biomedical sensing, environmental monitoring, food and agricultural applications, and application for security. The high flexibility offered by CNTs in the surface chemistry/functionalization, the possibility to integrate them in electronic device, the miniaturization of the sensor architecture and the high sensitivity offered are very important factors which are certain to play a role in a continuous evolution and commercialization of this technology. The rapid development of sensing methods based on CNTs indicate that superior responses are often obtained competing and likely surpassing most other sensor devices allowing application 
of this technology to protect and improve our environment, safety, and health. Some of the detection modalities in CNT based sensors are depicted in Figure 27.

(A)

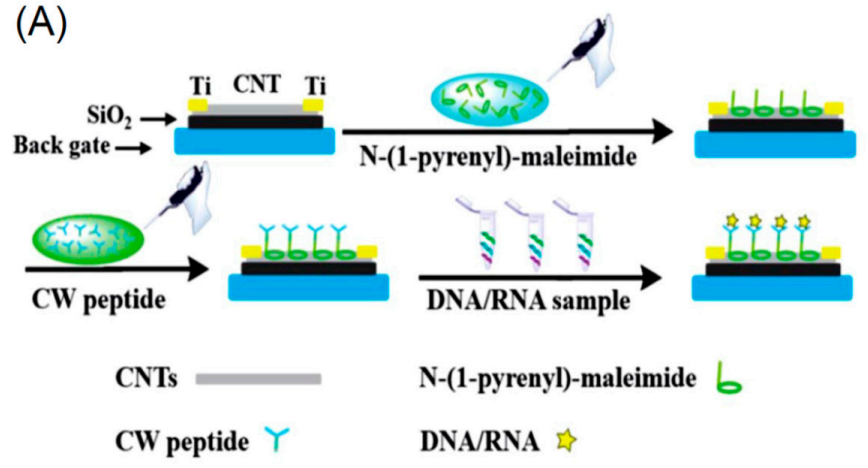

(B)

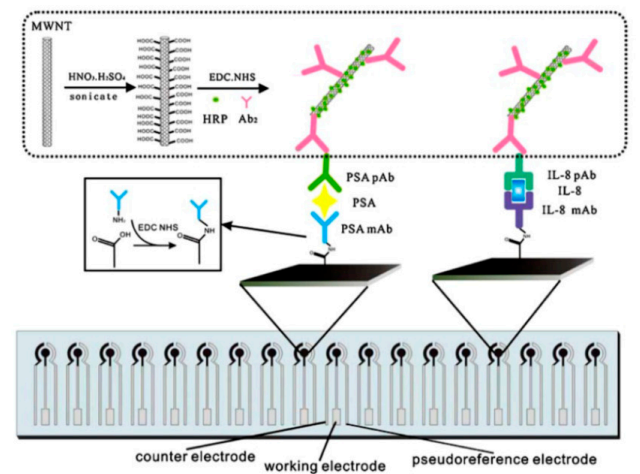

(C)
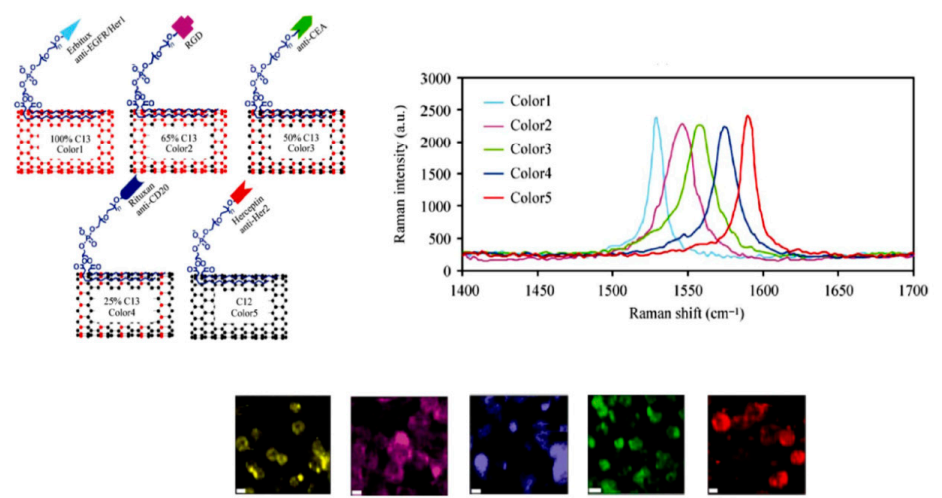

Figure 27. (A) Schematic representation of the novel peptide based CNT biosensor. Reprinted with permission from [490]. Copyright 2019 The Royal Society of Chemistry. (B) Schematic demonstration for the "sandwich" type strategy electrochemical immunosensor. A 16 channel screen-printed carbon electrode array was employed as the detection platform. MWCNT on the working electrode, were oxidized and functionalized with EDC/NHS + the capture antibodies PSA mAb or IL-8. The bonds between the target antigen (PSA or IL-8) to the antibody are recognized with an amperometric readout. Reprinted with permission from [498]. Copyright 2011 Elsevier. (C) Left: SWNTs with different Raman "colors". Color 1, 2, 3, 4, and 5 represent SWNTs with $\mathrm{C}^{13}$ isotope percentages of $100 \%, 65 \%, 50 \%, 25 \%$, and $0 \%$, respectively. Right: Raman spectra of the five different SWNT samples where the shift of SWNT Raman G-band peak is clearly dependent on the $\mathrm{C}^{13} / \mathrm{C}^{12}$ ratio in the SWNTs. Bottom: Five-color Raman imaging of cancer cells with different surface protein expression profiles were successfully labeled by SWNT tags and detected using Raman imaging (scale bars $=10 \mu \mathrm{m}$ ). Reprinted with permission from [499]. Coptright 2010 Springer.

\section{Graphene}

Graphene is a single layer of carbon atoms organized in a two-dimensional structure with a trigonal planar lattice. In graphene all the carbon atoms are $\mathrm{sp}^{2}$ hybrids characterized by $\mathrm{sp}_{\mathrm{xy}}$-mixed orbitals oriented along directions at $120^{\circ}$ while the remaining $\mathrm{p}_{z}$ orbital is orthogonal to the $x y$ plane. The three $\mathrm{sp}_{\mathrm{xy}}$ hybrids lead to the formation of hexagonal rings where each carbon atom is connected to other three carbon atoms via strong covalent $\sigma$ bonds. Differently, unhybridized $\mathrm{p}_{z}$-orbitals generate rather weak $\pi$ bonds formed by two lobes placed above and below the xy plane [43]. The $\sigma$ bonds of the hexagonal lattice have a length of $\sim 1.42 \AA$ (let us remind that the length of the strong $\sigma$ bonds of diamond is $\sim 1.54 \AA$ ). This mirrors the in-plane great mechanical strength of graphene, with breaking strength of $42 \mathrm{~N} \mathrm{~m}^{-1}$ and Young's modulus of 1.0 TPa [500]. Different from the strongly localized $\sigma$ bond electrons, the conjugated out-of-planar $\pi$ bond electrons are quasi-free. 
The particular structure of graphene leads to an electronic band structure with valence and conduction bands are cone-like structures touching each other near the Dirac points $\mathrm{K}$ and $K^{\prime}$ as shown in Figure 28.

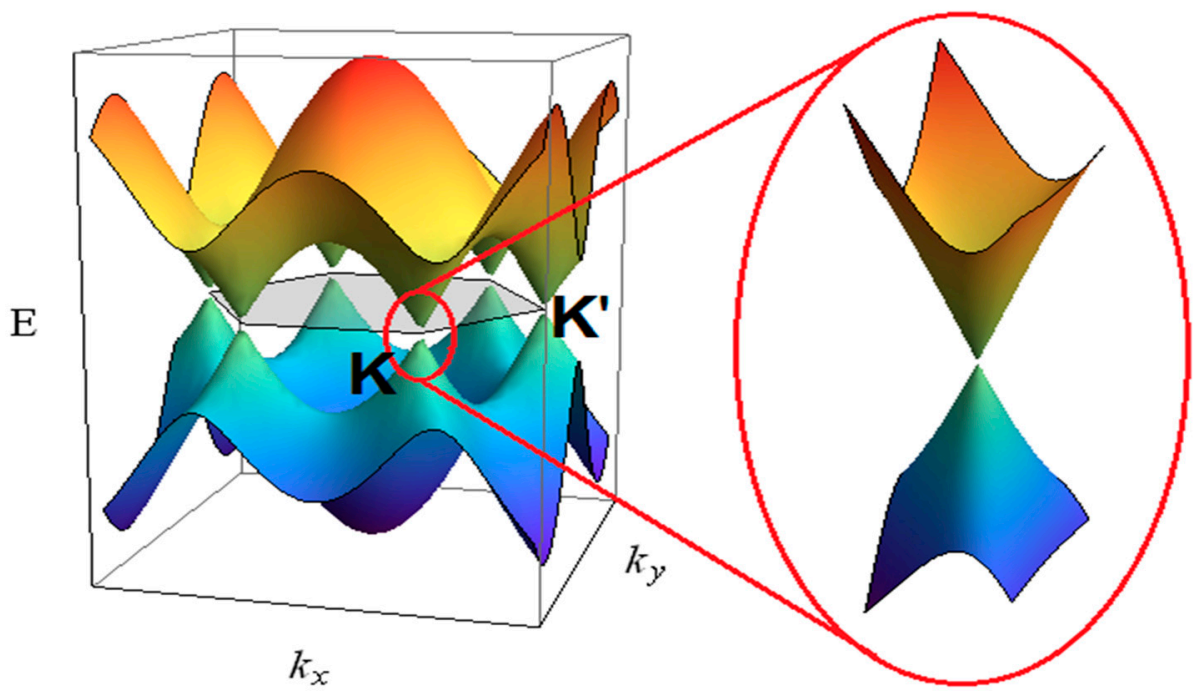

Figure 28. Band structure of graphene as a function of the momentum $k_{x}$ and $k_{y}$. One notices the valence band (at lower energy in blue) and the conduction band (at higher energy in orange). A zoom-in shows the bands close to the point where they touch each other in the Dirac point.

These features lead to two important consequences. First the free carriers in graphene follow a linear dispersion relation and behave as massless relativistic quasi-particles with unprecedented high mobility of $200,000 \mathrm{~cm}^{2} \mathrm{~V}^{-1} \mathrm{~s}^{-1}$ in suspended graphene and Fermi velocity of $\sim 1.10 \times 10^{6} \mathrm{~m} / \mathrm{s}$ and an electrical conductivity of $6300 \mathrm{~S} \mathrm{~cm}^{-1}$ [501]. Second, the two-dimensional honeycomb lattice of graphene displays a sublattice (chiral) symmetry [502]. The structural properties of single layer graphene are also mirrored by the high thermal conductivity of about $5000 \mathrm{Wm} \mathrm{K}^{-1}$ [503]. Finally, thanks to its single-atom thickness and 2D structure, graphene possesses an extraordinary high theoretical specific surface area of $2630 \mathrm{~m}^{2} \mathrm{~g}^{-1}$ [504]. However, despite graphene is a single atomic layer structure, it displays an unusual opacity absorbing a significant fraction of the incident light equal to $2.3 \%$ [505].

\subsection{Graphene Synthesis and Functionalization}

Despite the graphene structure is referred to the single sheet of carbon atoms, the name graphene is commonly used to indicate a class of materials formed by more stacked planes. The synthesis of graphene includes different methods resulting in materials possessing different properties e.g., layer number, lateral dimension, chemical residuals, surface charge and surface functional groups. Several synthesis methods have been developed to produce graphene and can be categorized in bottom-up and top-down approaches.

\subsubsection{Bottom-Up Synthesis}

In bottom-up approaches graphene is built from atom levels through three main synthesis methods: epitaxial growth on silicon carbide ( $\mathrm{SiC})$, chemical vapor deposition method (CVD) and plasma-enhanced chemical vapor deposition (PECVD). Epitaxial graphene growth on $\mathrm{SiC}$ is based on the high temperature thermal decomposition of $\mathrm{SiC}$ under vacuum or in inert gas [506]. In $\mathrm{SiC}, \mathrm{Si}$ and $\mathrm{C}$ are in 1:1 stoichiometry and the $\mathrm{Si}-\mathrm{C}$ bonds along the tetrahedral directions lead to a hexagonal layered structure where carbon and silicon are in alternating positions. Strictly speaking, the growth mechanisms of a graphene layer on the $\mathrm{SiC}$ surface is related to the difference in the vapor pressures of 
silicon and carbon. At high temperature the Si atoms sublimate while the remaining carbon atoms self-reorganize in the graphene structure with strong in-plane $\mathrm{sp}^{2}$ bonds [507].

This method was firstly reported in 1965 by Badami who annealed $\mathrm{SiC}$ in vacuum at $2280^{\circ} \mathrm{C}$ for an hour leading to the development of a graphite lattice crystal surface [508]. Successive studies suggested that a graphene monolayer already formed at $800{ }^{\circ} \mathrm{C}$ under ultra-high vacuum [509]. Authors also observed that graphite were formed on both Siand $\mathrm{C}$-faces of $\mathrm{SiC}$ but the $\mathrm{Si}$-face led to a monocrystalline graphite layer while the $\mathrm{C}$-face generated a polycrystalline graphite layer. However, the quality of the graphene obtained by this method is low because variable thickness, the small graphene grains caused by the changes in the surface morphology of $\mathrm{SiC}$ during sublimation. Figure 29A displays a typical graphene film grown on $\mathrm{SiC}$ : the formation of graphene is not a self-limiting process and with temperature Si sublimation proceeds with formation of a multilayer. To improve the graphene quality, $\mathrm{SiC}$ annealing was performed in an argon atmosphere [510] or in an external Si flux [511], or depositing a nickel [512] or cobalt [513] layer on SiC. Epitaxial graphene derived from $\mathrm{SiC}$ is useful to produce ready-to-use graphene for electronic applications. However, for other kinds of applications graphene transfer is required limiting the utility of this synthesis process.

CVD is a good alternative to the epitaxial growth on $\mathrm{SiC}$, to produce high quality, large area graphene although the properties of the produced graphene, e.g., number of layers and crystallinity, are affected by several factors such as precursors, catalyst layer, assistant gases, and temperature. In this CVD process, a hydrocarbon precursor gas introduced in the reactor chamber, is catalytically decomposed at high temperature [514]. The carbon radicals arrange into a graphene structure on the catalytic layer of the substrate. In CVD synthesis, methane is the most used precursor although other gasses as acetylene [515], ethylene [516], propene [517], or liquid precursors like methanol [518], ethanol [519], propanol [518], hexane [520], benzene [521], or even solid precursors like poly(methyl methacrylate) (PMMA) [522], amorphous carbon [523]. Deposition is made using different catalysts including nickel (Ni) [524], copper (Cu) [524], rhodium (Rh) [525], cobalt (Co) [526] or alloys [527-529]. The catalyst layer plays a role not only to lower the activation energy for the precursor decomposition, but also triggers different graphene growing mechanisms. For example, $\mathrm{Ni}$ induces carbon segregation and precipitation processes because at high temperature $\mathrm{C}$ has a high solubility in Ni. However, decreasing temperature decreases also the carbon solubility, carbon atoms diffuse out from the Ni-C solid solution and precipitate on the Ni surface producing a graphene films. $\mathrm{Cu}$ has much lower carbon solubility than $\mathrm{Ni}$ and only a small amount of carbon dissolves in this catalyzer. Thus, using $\mathrm{Cu}$ graphene grows directly from the precursor decomposition. In addition, the growth of graphene growth on $\mathrm{Cu}$ has the advantage to be a self-limiting process, which is important to form single layer graphene $[524,530]$. High resolution TEM structure of a CVD deposited graphene is shown in Figure 29B,C showing the perfect hexagonal planar structure of the carbon atoms observed at RT and $700{ }^{\circ} \mathrm{C}$ applied to desorb contaminants. The correspondent diffraction patterns are shown in the insets. A careful control of the CVD deposition parameters allows also the growth of large graphene single crystal shown in Figure 29D, also reaching macroscopic dimensions as in Figure 29E.

In PECVD processes the plasma decomposes the precursor allowing the synthesis of graphene at lower temperatures and shorter deposition times [531]. This method can overcome the evaporation problem of catalyst layer at high temperature. However, one drawback of the PECVD process is risk of the generation of plenty of defects in the graphene structure caused by the presence of the plasma [532]. 

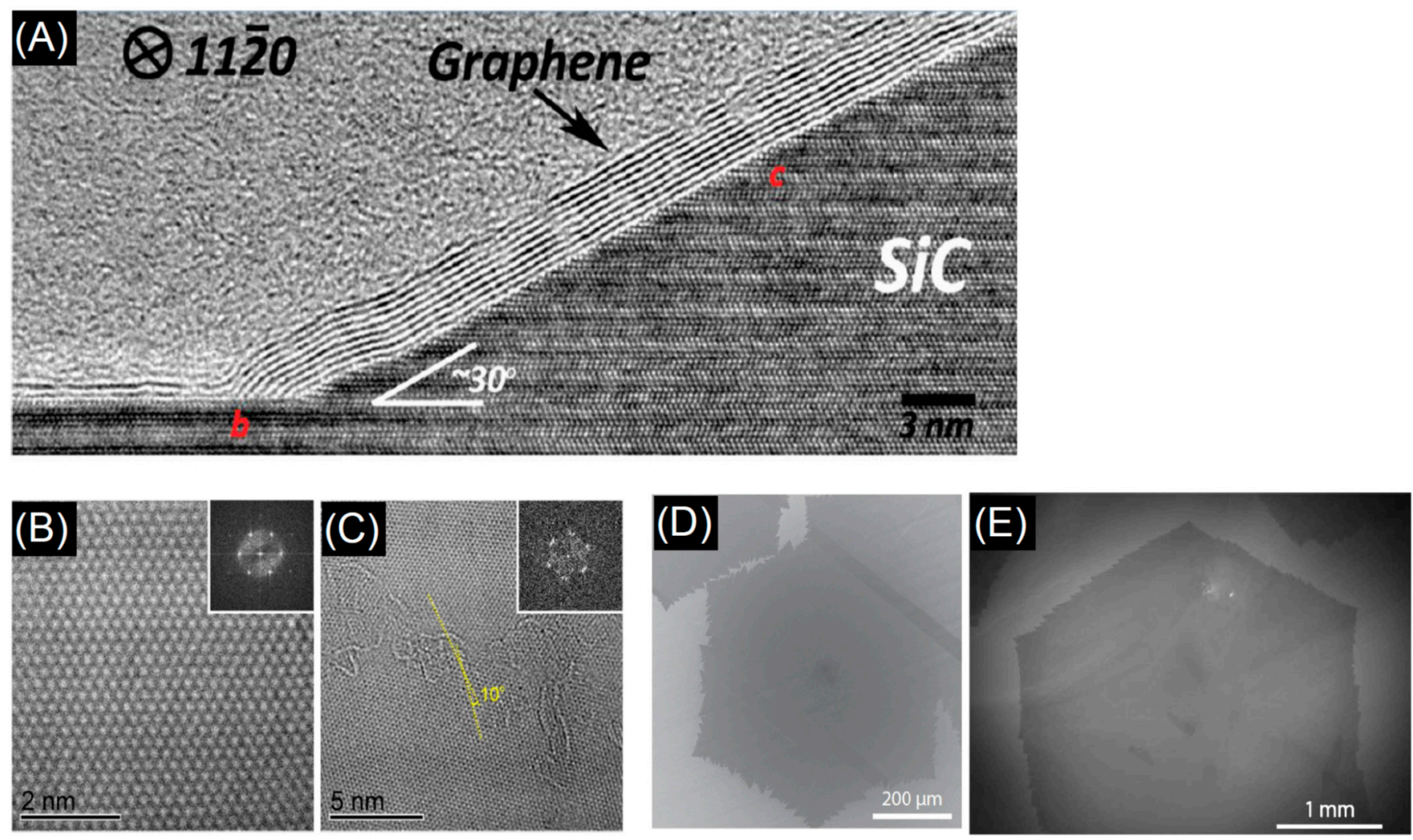

Figure 29. (A). TEM micrographs of epitaxially grown graphene on the (110n) plane of $\mathrm{SiC}$ at $1425^{\circ} \mathrm{C}$. Layer thickness appears not to be self-limiting, evidenced by the many-layer thickness (approximately 8) shown. Adapted with permission from [533]. Copyright 2010 American Chemical Society. (B) HR-TEM image of atomic structures of low temperature CVD grown graphene film and its corresponding diffraction patterns. (C) HR-TEM image near the graphene grain boundary. The misoriented angle between graphene domains is within 10 degrees and is seen as two adjacent diffraction patterns. (B,C) reproduced with permission from [534]. Large single crystal graphene produced inside a copper "pocket". (D) SEM image of an isolated graphene crystal with a diagonal dimension of nearly $750 \mu \mathrm{m}$; (E) SEM image of a CVD graphene single grain with a diameter of over $3 \mathrm{~mm}$. (D,E) reproduced from [535].

\subsubsection{Top-Down Synthesis}

In the top-down synthesis, graphene sheets are detached from high-quality graphite crystals using mechanical or chemical methods namely the mechanical exfoliation method, the chemical exfoliation method followed by reduction of oxidized graphene.

The mechanical exfoliation was the first documented method to successfully separate graphene from crystalline graphite. Exfoliation can be made using a simple adhesive tape to break the van de Waals forces between graphite layers [536]. High quality graphene is produced using this method which, however, is not scalable. Graphene sheets are also produced by applying ultrasonic force to graphite in solvents as $\mathrm{N}$-methylpyrrolidone (NMP) which relies on the strong interactions between NMP and graphene sheets [537]. Another mechanical exfoliation method is the use of the shear force in the ball-milling process. Ball-milling can be performed in both wet [538] and dry form [539]. A common problem of ultrasonic and ball-milling methods is the presence of some un-exfoliated graphite mixed with graphene sheets difficult to eliminate. In addition, ball-milling also produces undesired amorphous carbon domains and defects in the graphene sheets.

Chemical exfoliation is a versatile method for the large-scale synthesis of graphene in a two steps process: (i) production of oxidized graphene (GO) sheets, and (ii) oxygen removal from GO via reduction processes. To generate GO, Hummer's method is usually used exfoliate crystalline graphite. The exfoliation process utilizes sodium nitrate, concentrated sulfuric acid and potassium permanganate, leading to strongly oxidized graphene 
sheets [540]. Graphene oxide layers can be separated by ultrasonic treatment in polar solvent, especially in water, due to the enlarged layer spacing introduced by the oxygen functional groups. Finally, the graphene structure can be restored using electrochemical reduction [541], thermal reduction [542], chemical reduction [543] or hydro/solvothermal method [544]. Figure 30A,B displays the structure of graphene oxide obtained by exfoliation. TEM shows the presence of defects which appear more clearly in Figure 30C,D. Chemical reduction is widely for GO reduction and a variety of reductant were studied. There are several reductants which are effective for GO reduction to rGO, e.g., hydrazine [545], sodium borohydride [546], hydrohalic acid [547], ascorbic acid [548]. The selection of the appropriate reductant is linked to the kind of application envisaged. Chemical residuals from reductants may affect the biocompatibility of the final product since most of them are toxic. To solve this problem, recently green reductants as plant extracts, sugars, microorganisms and amino acids have been tested [549]. As an example, nicotinamide adenine dinucleotide phosphate (NADPH) contained in baker's yeast was utilized to reduce and, at the same time, functionalize the rGO. Specifically, the amine groups of NADPH couple with the epoxy functionalities of GO resulting in a stable water suspension of yeast-rGO [550]. The structure of reduced GO is shown in Figure 30E while in Figure 30F are highlighted the different defects present on the graphene flakes after the reduction process.
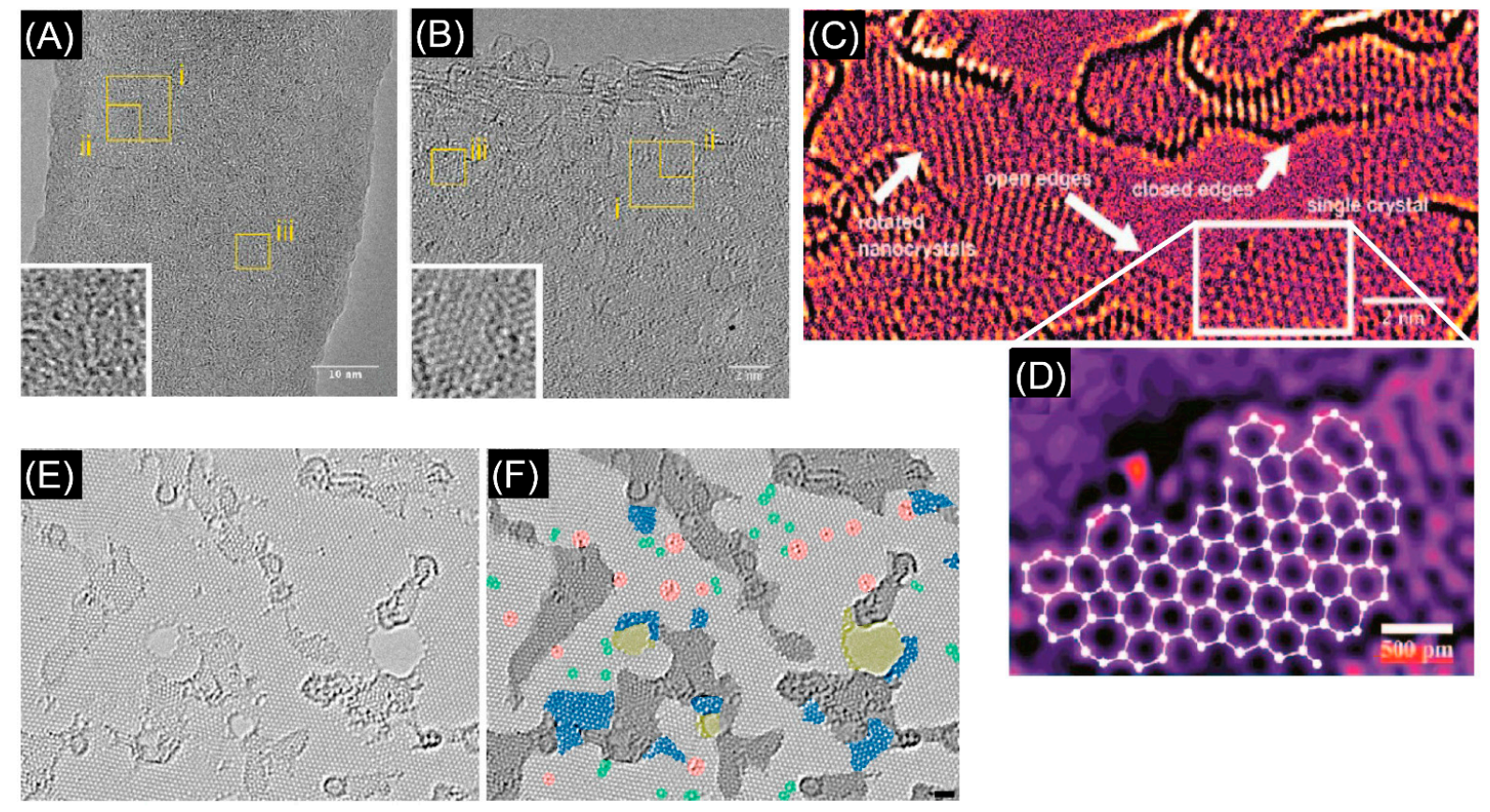

Figure 30. (A) Graphene oxide observed at room temperature and (B) at $700{ }^{\circ} \mathrm{C}$ which in AC-TEM appear notably different. (C) a region of graphene oxide in false color to enhance contrast. Both open (monolayer, low contrast) and closed (folded over bilayer, high contrast) edges are visible as are regions of disorder that separate the nanocrystallites from each other. (D) shows the atomic structures of the region of monolayer GO indicated in (C). (A-D) reprinted with permission from [551]. Copyright 2016 American Chemical Society. (E) TEM image of a single layer reduced-graphene oxide membrane (F) with color added to highlight the different features: defect free crystalline graphene in light gray; contaminated regions in dark gray; disordered single-layer carbon networks, or extended topological defects in blue; red areas highlight individual ad-atoms or substitutions; green areas indicate isolated topological defects. Reproduced with permission from [552]. Copyright 2010 American Chemical Society.

\subsection{Graphene Functionalization}

As for the other carbon nanostructures, chemical functionalization of graphene is one of the crucial steps of material preparation determining the final properties and the kind of applications namely electronics, filler in nanocomposite synthesis, biomateri- 
als/biomedicine, sensing, energy, environment. Functionalization transforms the zero-gap graphene in a semiconductor suitable for electronic applications [553]. In the case of biomaterials, functionalization renders graphene soluble in water, an appropriate surface chemistry renders graphene a suitable nanoplatform for drug delivery, such as tissue engineering and other applications [554].

Graphene is broadly utilized as the ultimate electrode material for various electrocatalytic applications. Modification of the surface chemistry with appropriate functional groups makes the resulting electrode more efficient than the individual components [555].

The chemistry of graphene resembles that of graphite and other $\mathrm{sp}^{2}$ hybridized carbon allotropes [556]. As in CNTs, also in graphene the presence of defects play an important role. Atoms at the edges of defects are generally more reactive than the surrounding $\pi$ surface although functionalization of graphene may occur also on the $\pi$ surface [557] as we will describe later on. As for CNTs, also in the case of graphene functionalization may be performed by covalent or non-covalent routes.

\subsubsection{Covalent Functionalization}

Among the chemical modifications of the graphene surface, those regarding the graphene oxide and the reduction processes have been extensively studied. This enables grafting of a large number of different atoms/organic groups on pristine graphene, radical additions, electrophilic substitution, and cycloaddition reactions. Talking about graphene oxide (GO) studies regarding this material dates back to the 18th when Brodie demonstrated the possibility of obtaining graphene oxide from graphite using potassium chlorate and fuming nitric acid [558]. An alternative was proposed in the sixties by Hummers, and Hoffman who used permanganate and concentrated sulfuric acid or a mixture of sulfuric and nitric acids, achieving similar levels of oxidation of graphite. However it has been demonstrated that the oxidation level depends not only on the nature of the oxidants but also on the quality of the graphite utilized and on the reaction conditions [559]. These scalable processes lead to the formation of hydroxyl and epoxides, and smaller amounts carboxy, carbonyl, phenol, lactone, and quinone groups [560] (see Figure 31A at the sheet edges groups breaking the continuity of the aromatic lattice and inducing a change of the $\mathrm{C}$ atom's hybridization [561]. Thanks to the high concentration of polar functional groups, GO is readily dispersible in water. GO is a functional group rich material, but it may be considered as a starting substance for the preparation specific compounds. Moreover, the hydrophilicity of GO allowed its uniform deposition on different substrates as thin films, necessary for applications in electronics [108]. The presence of oxygen based functional groups allows to further tune the surface chemistry adding desired functional molecules. In [562] a selective functionalization of the hydroxyl groups was carried out using an esterification reaction with aminocaproic acid (see reaction scheme in Figure 31A) and the Williamson reaction with tert-butyl (4-iodobutyl)carbamate. Selective derivatization of Ketones by the Wittig reaction was also studied. The high surface area GO may be used as a support for nanoparticles dispersion, to maintain their electrochemical activities maximizing the electron transfer and also providing optimal mass transport of the reactants to the electroactive site. Nanoparticles are made of metal or metal oxides and are attached to the GO substrate for metal nanoparticles (such as $\mathrm{Pt}, \mathrm{Au}, \mathrm{Ru}, \mathrm{Ag}, \mathrm{Cu} . .$. ) and oxide nanoparticles $\left(\mathrm{TiO}_{2}, \mathrm{ZnO}, \mathrm{SnO}_{2}, \mathrm{Cu}_{2} \mathrm{O}, \mathrm{MnO}_{2}, \mathrm{Mn}_{3} \mathrm{O}_{4}, \mathrm{NiO}, \mathrm{SiO}_{2}\right)$ have been described for the fabrication of electrodes based on GO [563-566]. Moreover, quantum dots are utilized to functionalize GO for producing platforms for electrochemical applications $[567,568]$. Other species utilized to functionalize GO are Prussian blue [569], metal hydroxides [570] used to modulate the electrochemical properties. There is a wide group of organic compounds used to functionalize GO for example porphyrins [571], aromatic dyes [572], alkylamines [573], ionic liquids [574], pyrene and perylenediimide [575], cyclodextrin [576], aryl diazonium compounds [577] and polymers [578].

Graphene sheets are also hydrogenated. Hydrogenation converts $\mathrm{sp}^{2}$ into $\mathrm{sp}^{3}$ hybrids. This induces significant changes in the electronic properties converting graphene from a 
highly conductive into an insulating material [579]. RF-CVD was used to functionalize graphene in a hydrogen atmosphere [580]. Other research groups studied the wet chemical routes as the Birch reduction for the preparation of hydrogenated graphene [581]. Change of the electronic properties of graphene is performed also with doping with halogenated elements [582]. As it happens for hydrogen, covalently bound halogens (graphene halides) strongly modify the graphene properties. For example, fluorographene is only stable stoichiometric graphene halide and the thinnest insulator. Halogenated graphenes exhibit a plethora of remarkable and interesting electronic, optical, thermal, electrocatalytic, magnetic, mechanical, biological, and chemical properties in comparison with their graphene counterparts [582].

Addition of organic functional groups is also possible and has been developed for example to render graphene sheets dispersible in common organic solvents, which is of pivotal importance for the production of nanocomposite materials (see Figure 31B). Radical addition to $\mathrm{sp}^{2}$ carbon hybrids is one of the routes utilized to overcome the low reactivity of pristine graphene. The radical addition of phenyl species obtained by heating of aryl diazonium salts is suitable option to form new covalent bonds to graphene [583]. The aryl diazonium salt covalent modification allows the preparation of functionalized graphene with refined dispersibility in polar organic solvents and water by modifying the substituents in the aryl-ring. This kind of functionalization was utilized for developing high performance graphene-based nanocomposites, with enhanced dispersibility of graphene nanosheets in polymer hosts leading to strong interlayer cohesive energy and surface inertia [584] (see Figure 31C). Covalent modification using 4-bromobenzene diazonium tetrafluoroborate (4-BBDT) was utilized to change the electronic properties of graphene [585]. Authors estimated a downward shift of the Fermi level dependent on the 4-BBDT molecule concentration thus offering the possibility to tune the electronic properties of graphene. The change of electronic properties was made using the diazonium based functionalization which results in a charge transfer which opens a bandgap transforming the graphene regions from quasimetallic to semiconducting and insulating [586]. Click chemistry is possible to perform highly regioselective, rapid and easy and efficient reaction to functionalize the graphene surface. Graphene was functionalized with 4-propargyloxybenzenediazonium tetrafluoroborate and a subsequent attachment by click chemistry of a short chain polyethylene glycol with terminal carboxylic end group [587]. The functionalization resulted in an increased $\zeta$ potential of the particulate thus increasing the wettability of the graphene. Another kind of covalent functionalization is the reaction of azomethine ylides with $\mathrm{sp}^{2}$ graphitic carbon atoms (see Figure 31D). This reaction has been successfully applied to carbon nanostructures as fullerenes, CNOs, CNTs, and also to graphene [588] despite the lack of surface curvature. This reaction, the Prato reaction, consists in a 1,3-dipolar cycloaddition reaction of azomethine ylides generated in situ by thermal condensation of aldehydes and $\alpha$-amino acids [101]. Authors demonstrated that the reaction proceeds not only at the edges possessing higher reactivity but also at the internal carbon-carbon bond network. The Prato reaction is utilized to obtain a number of organic derivatives useful for different applications.

Graphene sheets were obtained from a dispersion of graphite in pyridine [589]. Dihydroxyphenyl groups perpendicular to the graphene surface were formed by the addition of azomethine ylide precursors. The presence of hydroxyl groups induced higher dispersibility of the functionalized graphene in polar solvents. The Prato reaction was utilized to add particular molecules while maintaining the graphene electronic transport. So, the basal plane of few-layer graphene was modified with 1,3-dipolar cycloaddition of azomethine ylides and the subsequently coupled covalently with an electron-donating phthalocyanine used for light-harvesting [590]. The system showed a very fast charge separation between phthalocyanine and the few-layer graphene and a slow recombination. It has been reported the graphene functionalization by nitrene chemistry based on different perfluorophenyl azides. Phenyl and alkyl azides can react with the $\mathrm{C}-\mathrm{C}$ bonds of graphene by the formation of the reactive nitrene intermediate $([2+1]$ cycloaddition to the double bonds of 
graphene, forming an aziridino-ring) [591]. Depending on the functional group bonded to the perfluorophenyl azides the final product was soluble in water or organic compounds. The reaction is applicable regardless graphene size, shape, or configuration thus improving the processability, also considering the possibility to furthermore derivatize with additional molecules opening a number of opportunities in graphene-based materials. Alkyl azides, alkyl groups such as hexyl, dodecyl, hydroxyl-undecanyl, and carboxy-undecanyl have been inserted into surfactant-wrapped graphene sheets [592] allowing bonding gold NPs to the graphene surface used to visualize the reactive sites. Other possible reactions are the Bingel-Hirsch reaction [593], a nucleophilic [2+1] cyclopropanation of a double bond, usually used on fullerene but successfully applied also to graphene (see Figure 32A). The reaction proceeds reacting a bromomalonate in the presence of a base. In [594] graphene obtained by exfoliation was mixed to 8-diazabicyclo[5.4.0]undec-7-ene (DBU), carbon tetrabromide ( $\mathrm{CBr} 4)$, and the corresponding malonate. A Bingel reaction was activated by applying microwaves leading to cyclopropanate malonate units covalently grafted onto the honeycomb skeleton. The final product was a stable suspension for several days in a variety of organic solvents.
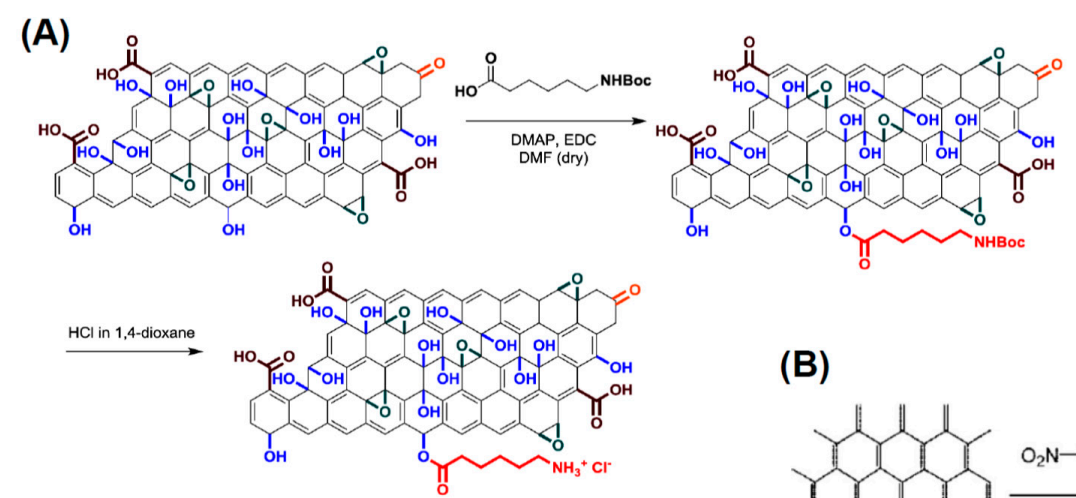

(B)

(C)
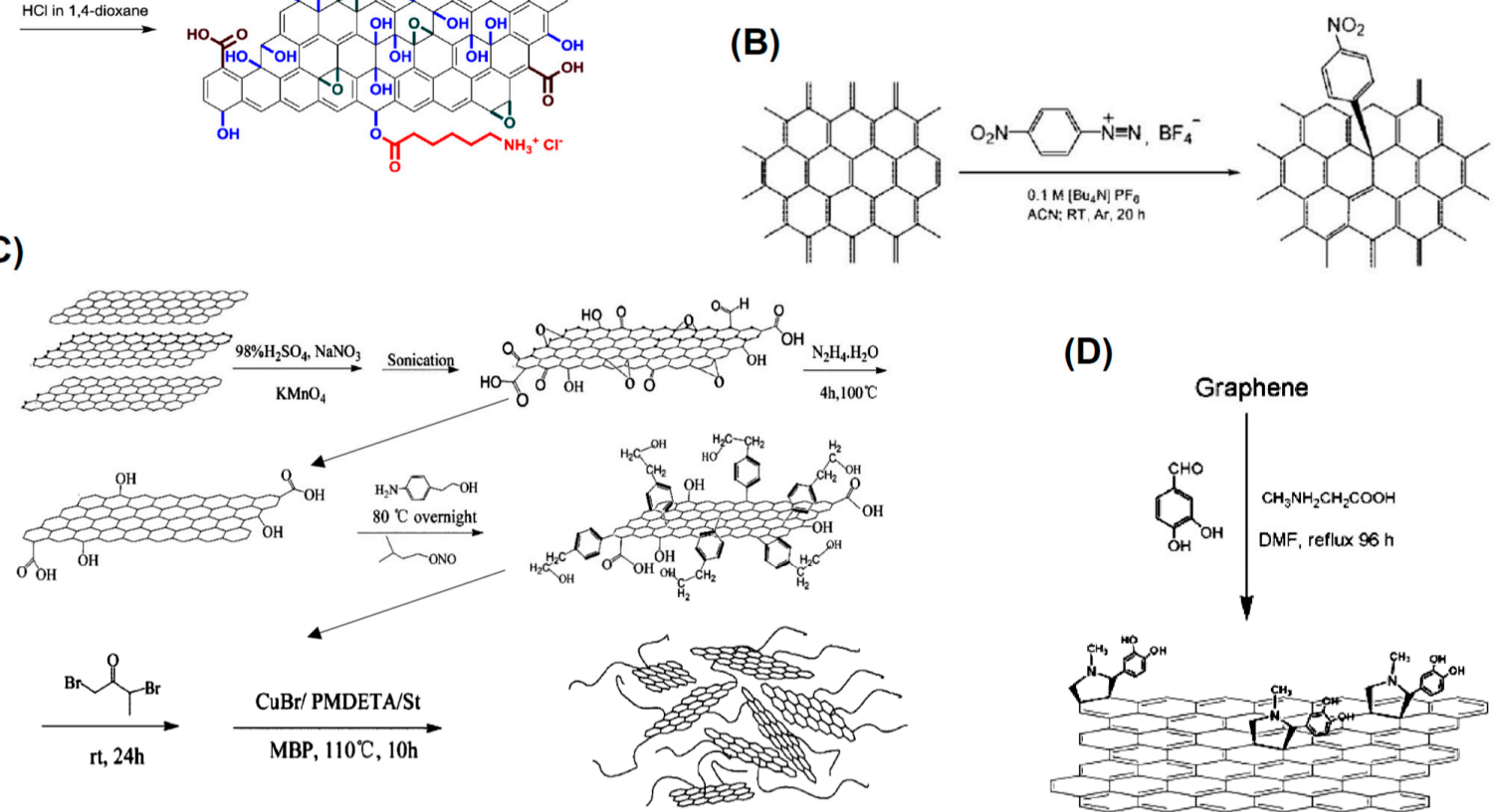

(D)
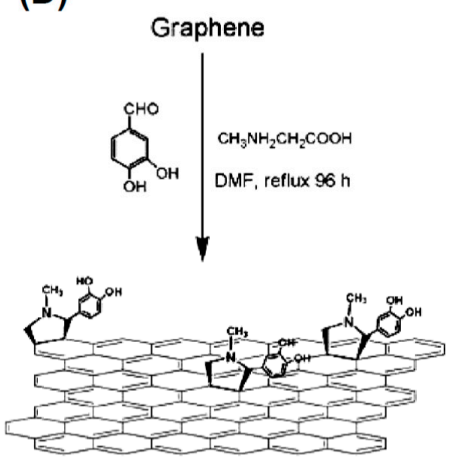

Figure 31. Some examples of graphene functionalization. (A) Possible oxide functional groups of graphene; esterification of the GO hydroxyl groups performed using Boc-protected aminocaproic acid in the presence of EDC and DMAP as coupling agents; Boc cleavage performed using $\mathrm{HCl}$ in 1,4 dioxane, leading to amine functionalized GO. Reprinted with permission from [562] Copyright 2018 Institute of Physics. (B) Schematic illustration of the spontaneous grafting of aryl groups to epitaxial graphene via reduction of 4-nitrophenyl diazonium (NPD) tetrafluoroborate. Adapted with permission from [586]. Copyright 2009 American Chemical Society. (C) Covalent polymer functionalization of graphene: synthesis route of polystyrene-functionalized graphene nanosheets. Reprinted with permission from [584]. Copyright 1991 Royal Society of Chemistry. (D) Schematic representation of the 1,3 dipolar cycloaddition of azomethine ylide to defect-free graphene. Adapted with permission from [589]. Copyright 1996 Royal Society of Chemistry. 


\subsubsection{Non-Covalent Functionalization}

In noncovalent functionalization the linkage between graphene and the functional group is weak enough to preserve the intrinsic electronic structure of graphene. In noncovalent functionalization the interaction of the countermolecules to the hexagonal lattice preserves the intrinsic chemical and physical characteristics while adding interesting selective properties. This kind of functionalization is based on the so called $\pi-\pi$ interaction established between aromatic rings. There are several molecules that can be utilized in non-covalent functionalization. Pyrene moiety has attracted great interest because the strong affinity toward the graphite basal planes. Graphene was functionalized with 1pyrenebutyrate to increase the aqueous dispersibility of graphene in aqueous medium [595]. In another work $\pi-\pi$ noncovalent functionalization was used to attach pyrenebutanoic acid succinimidyl ester to graphene obtaining an enhancement of the power conversion efficiency in graphene-based photovoltaic devices [596]. Unique optical and sensing properties were obtained with a 1-pyrenecarboxylic acid functionalization of graphene [597]. Other examples of non-covalent functionalization may be found in [598]. Some examples of $\pi-\pi$ interaction are shown in Figure 32B.
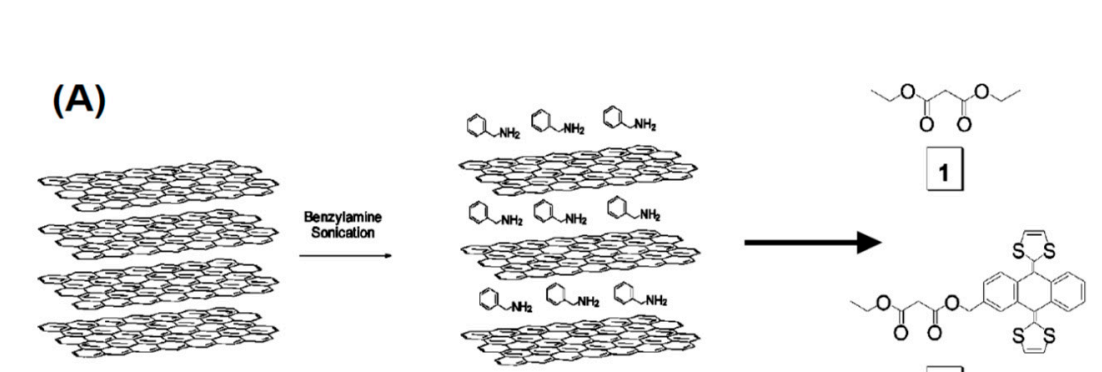

1 or $2 \underset{\mathrm{MW} \text { irradiation }}{\mathrm{CBr}_{4}, \mathrm{DBU}}$

(B)
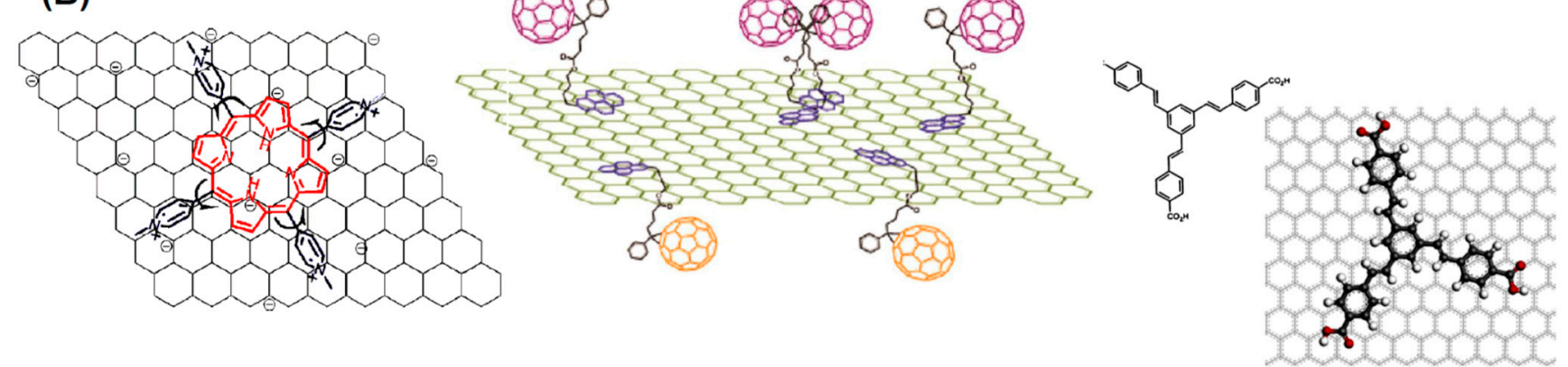

Figure 32. (A) Left: exfoliation of graphite flakes by sonicating in benzylamine; (right) functionalization of graphene by a Bingel reaction using 1,8-Diazabicyclo[5.4.0]undec-7-ene (DBU) and CBr4 and diethylmalonate. Adapted with permission from [594]. Copyright 2010 American Chemical Society. (B) Examples of non-covalent $\pi-\pi$ interaction. Left, the four cationic methylpyridinium molecules (blue) are used to bind a porphirin moiety (red) to a graphene sheet. Reprinted with permission from [599]. Copyright 2009 American Chemical Society. Center Schematic $\pi-\pi$ interaction used to bind rGO-pyrene-PCBM spacer to attach $\mathrm{C}_{60}$ far from the graphene sheet. Reprinted with permission from [600]. Copyright 2013 American Chemical Society. Right structure of Ramizol- (1,3,5-tris[(1E)-20-(40-benzoic acid) vinyl]benzene and its interaction with a graphene sheet. Reprinted with permission from [601]. Copyright 2015 Elsevier.

\subsection{Graphene Sensing}

Advantageous utilization of graphene to develop sensing devices is due to the distinct properties of this material as the exceptional specific surface area, the unique optical 
properties, excellent electrical and thermal properties, the high strength and the flexibility. Notably, these properties persist also in the double and multi-layered graphene structures. A supplementary important feature of the graphene platform is the possibility to adapt its structure and the working conditions accordingly to the application. In electrochemical sensors, the functionalization and the surface engineering pay a crucial role in coupling the analyte molecule to the electrode surface. As for strain, among the two-dimensional materials graphene is the strongest being able to sustain reversible tensile elastic strain larger than $20 \%$. This feature enables the possibility use graphene as a sensor being its properties changed by strain. This fact derives from the strain induced modification of the electronic structure as the shift of the Dirac cones [602] and the generation of a pseudomagnetic field and a reduction of the Fermi velocity [603]. Electron phonon coupling is also sensitive to the application of strains. In particular under the application of uniaxial strains, the $G$ band redshifts and splits into two single $G+$ and $G-$ bands $[604,605]$. The $G+$ and $\mathrm{G}-$ band splitting as a function of the applied uniaxial strain is shown in Figure 33. The Raman scattering from the $\mathrm{G}+$ and $\mathrm{G}-$ bands shows a distinctive polarization dependence on the angle between the strain direction and the underlying graphene crystal axes. The $\mathrm{G}+$ and $\mathrm{G}$ - relative intensities vary with polarization, thus allowing to obtain the strain direction with respect to sample crystallographic orientation.
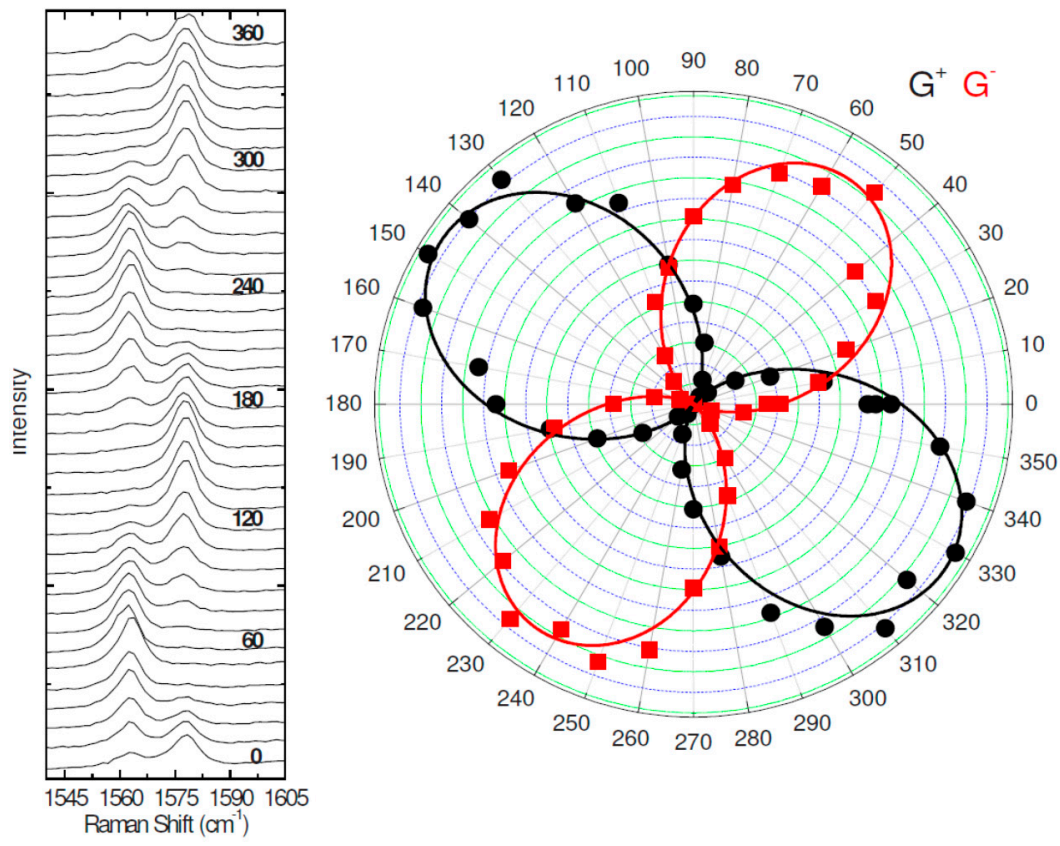

Figure 33. Raman spectra (left) and polar plot (right) of the fitted G+ and $\mathrm{G}^{-}$peaks as a function of the angle between the incident light polarization and the strain axis $\theta_{\text {in }}$ measured with an analyzer selecting scattered polarization along the strain axis $\theta_{\text {out }}=0$. Reprinted with permission from [605]. Copyright 2009 American Physical Society.

These graphene properties may be utilized to derive information about the applied strain. Another important parameter is the gauge factor reflecting the efficiency in generating a change in the electric properties of the composite material based on mechanical deformations. In [606], authors mixed graphene with natural rubber to create conducting composites. The material displayed excellent strain sensing capability with a $10^{4}$-fold increases in resistance and working at strains exceeding $800 \%$. In another work sensors were fabricated by depositing graphene flakes on flexible plastic supports [607]. The change of the flake-flake overlap for different percolation networks upon application of a strain explains the changes in the gauge sensitivity of the material associated to the superior sensitivity of the sensor (see Figure 34). Flexible plastic or stretchable rubber substrates 
were also used to fabricate graphene based piezo-resistive strain sensor [608]. The sensor properties were studied upon applied strains up to $7.1 \%$. The sensor performances were tested on a glove showing the ability to measure magnitudes and directions of the principal strains on the glove induced by the motion of fingers. A film of GO was deposited on a PET coated DVD and was reduced using a Light-Scribe DVD burner [609]. Under substrate deformation the device showed a good linear response to strain and good multi-cycle operation opening perspectives for wide applications in medical-sensing, bio-sensing, artificial skin and many other areas.

(A)

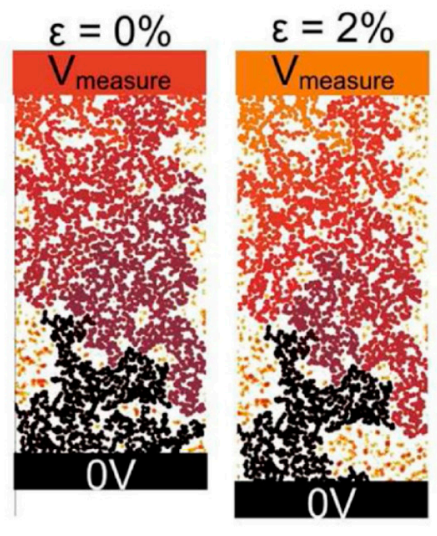

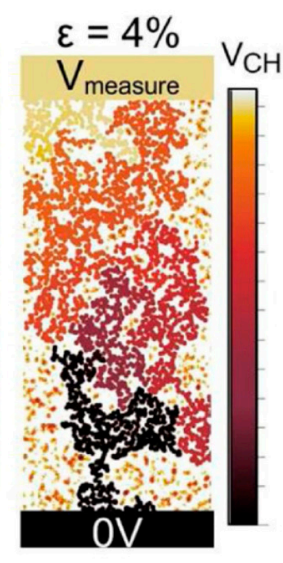

(B)

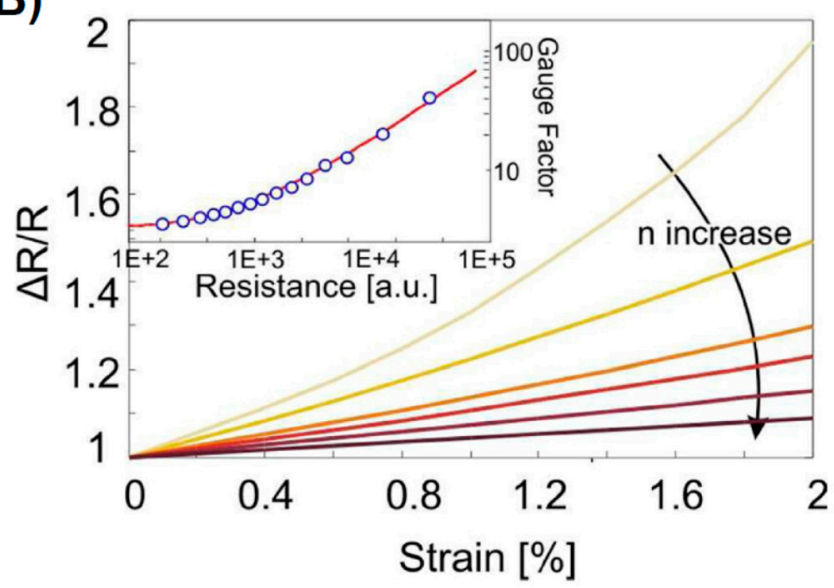

Figure 34. Modeling of percolation through graphene flake network under strain. (A) Representation of voltage drop at fixed current in a graphene film at different levels of strain. (B) Resistance-strain diagram for different graphene flake number density n. (inset) Gauge factor GF as a function of unstrained resistance R0. Reproduced with permission from [607]. Copyright 2012 American Chemical Society.

To overcome the fragility, reduced graphene oxide aerogels were mixed to water soluble polyimide leading to formation of superflexible 3D architectures [610]. The $\mathrm{rGO} /$ polyimide was produced by freeze casting and thermal annealing. The resulting monoliths exhibited as low density, excellent flexibility, super-elasticity. The high recovery rate, and extraordinary reversible compressibility were exploited for sensing strains. The sensor showed perfect linearity: a compression strain of 10\%, resulted in an electronic resistance change of $9.96 \%$. Interestingly, graphene strain sensors may be fabricated using textiles [611]. The sensing properties are obtained by using graphene as a filler in the textile fibers to obtain conduction properties or the using graphene served as conductive coatings. The response of the sensors shows a linear behavior only within a limited strain range due to the slip inside the fiber or fabric and the change of textile strain sensor structure [611]. Although the properties of the graphene-based textile sensors have to be improved, they have important potentialities in many sectors as flexible and wearable sensors for electronic skin, human-machine interfaces, human activities monitoring, intelligent robots, and human health detection.

Detection of substances based on graphene is an extremely broad area of research either for the number of molecules probed and for the different methods used. Hereafter we will review only the electrochemical graphene-based sensors.

A broad area of sensing is based on the electrochemical reactions developed at the electrode/analyte interface. A material is suitable as electrochemical sensor if it possesses a large electrochemical potential window, good electron transfer rate, appropriate redox potentials. Graphene has a wide electrochemical potential window of ca. $2.5 \mathrm{~V}$ in $0.1 \mathrm{M}$ PBS at pH 7.0 [612], which is comparable to that of graphite, GCE [612,613], but possesses a charge-transfer resistance much lower than these electrodes [612]. Finally, the peculiar 
electronic structure of graphene, and the high density of the electronic states over a wide energy range are the reasons for the graphene fast electron transfer [613].

Among the possible sensing application of graphene those related to the detection of biomolecules is one of the more important. In this respect, much attention was paid to the glucose sensing. As for the CNTs, also in the case of graphene enzymatic detection of glucose is based on glucose oxidase $\left(\mathrm{GO}_{\mathrm{x}}\right)$. To increase the device sensitivity, GO sheets were decorated with gold nanoparticles through a benzene $(\mathrm{Ph})$ bridge employing aryldiazonium salt chemistry [614]. The functionalized GO sheets were then attached to a 4-aminophenyl functionalized GCE. Finally, the GOx enzyme was attached to the gold NP through a 4-carboxyphenyl linker (see Figure 35). The sensor showed a linear behavior in the range of $0.3-20 \mathrm{mM}$ with a sensitivity of $42 \mathrm{~mA} \mathrm{mM}^{-1} \mathrm{~cm}^{-2}$ for glucose detection and a fast electron transfer and enzyme turnover rates of 8.3 and $112 / \mathrm{s}$, respectively. This sensor was characterized also by a high selectivity for glucose showing an almost null amperometric response when in contact with $100 \mathrm{mM}$ of interfering analytes as dopamine, ascorbic acid, uric acid, acetaminophen, fructose, lactose, and galactose. In an attempt to increase the sensitivity, newer sensors try to implement a direct electron transfer allowing amperometric measurement of the glucose level. In some sensors the GOx enzyme to be directly attached to the sensing electrode [615] reaching a LOD of $1.7 \mu \mathrm{M}$ with a linear response between 0.02 and $3.2 \mathrm{mM}$. However, an efficient direct electron transfer between the biocatalyst and the electrode surface remains a key challenge.

\section{Gluconic}
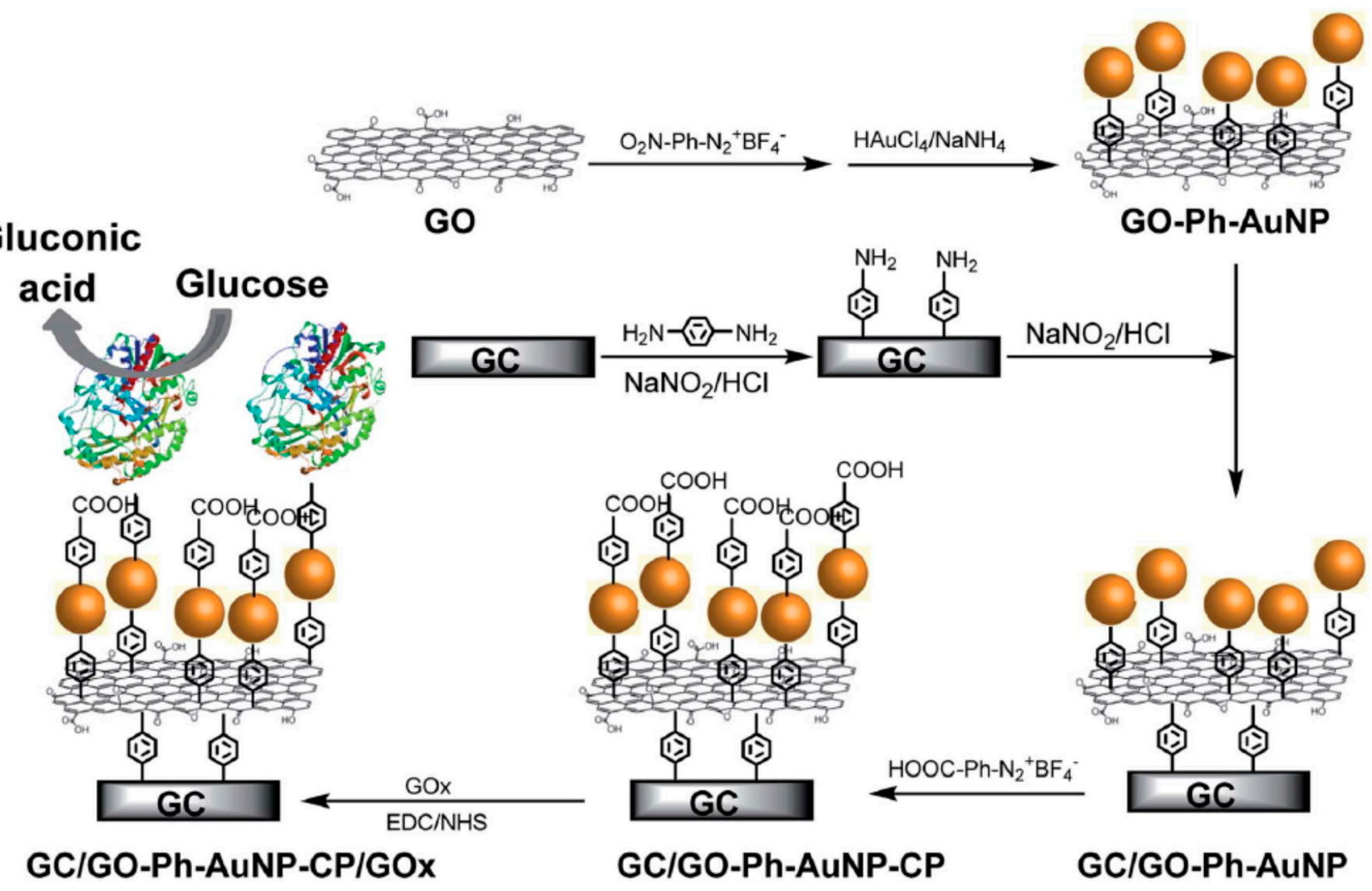

Figure 35. Scheme of a glucose biosensor based on AuNP loaded GO nanocomposites (GO-Ph-AuNP). Based on aryldiazonium salt chemistry, AuNPs were decorated to GO through a benzene bridging. GO-Ph-AuNP was then attached to the 4-aminophenyl modified GCE by C-C bonding. The formed GC/GO-Ph-AuNP interface was further modified with 4-carboxyphenyl before covalent attachment of glucose oxides (GOx) by amide bonds to achieve the GC/GO-Ph-AuNPCP/GOx sensing interface. Reproduced with permission from [614]. Copyright 2016 Royal Society of Chemistry.

Increased efficiency was obtained using the flavin adenine dinucleotide (FAD) as mediators in the redox reactions [616]. Other researchers tried to increase the sensing 
efficiency by tailoring the electronic properties of the electrode. As an example a GCE was engineered with a direct electrochemical reduction of single-layer $\mathrm{GO}$ and subsequent functionalization with (3-aminopropyl)triethoxysilane (APTES) used to immobilize GOx by covalent bonds. The GCE-APTES-rGO-GOx showed a linear behavior in a range 0 to $24 \mathrm{mM}$ glucose [617]. There are a series of different attempt to fabricate sensors with improved sensing capability. In [515] used good performances were obtained using a CVD-grown graphene, in other works the sensing electrode was coated with a Layer-by-layer deposition of graphene and GOx through $\pi-\pi$ interaction [618] or immobilization of GOx on a porousgraphene structure [615]. Other sensors are based on Graphene/polymer composites as for example polyethylene glycol [619], polyvinylpyrrolidone [620], or chitosan [621]. A final note concerns the research made to overcome degradation of the GOx enzyme induced by $\mathrm{pH}$, temperature, pressure or humidity. Non-enzymatic glucose sensors can be fabricated using nitrogen-doped graphene-encapsulated nickel cobalt nitride $(\mathrm{NiCo} 2 \mathrm{~N})$ core-shell nanostructures [622]. The sensor displayed a linear behavior in a wide range from $2.008 \mu \mathrm{M}$ to $7.15 \mathrm{mM}$, an excellent sensitivity of $1803 \mu \mathrm{A} \mathrm{mM}^{-1} \mathrm{~cm}^{-2}$, and a low detection limit of $50 \mathrm{nM}$. In addition, the $\mathrm{NiCo} 2 \mathrm{~N}$ decorated graphene electrode showed a remarkable $\mathrm{H}_{2} \mathrm{O}_{2}$ sensitivity of $2848.73 \mu \mathrm{A} \mathrm{mM}^{-1} \mathrm{~cm}^{-2}$, a wide detection range of $200 \mathrm{nM}$ to $3.4985 \mathrm{mM}$, and a LOD of $200 \mathrm{nM}$ coupled to a short response time of $<3 \mathrm{~s}$. Glucose was detected alsu using nitrogen-doped graphene decorated with copper nanoparticles [623], displaying a linear response in the range $0.004-4.5 \mathrm{mM}$ and a LOD of $48.13 \mu \mathrm{AmM}^{-1}$. Another possibility is the use of Pt nanoparticles supported on GO hydrogel [624]. In this case the electrode showed a linear behavior in the range $5-20 \mathrm{mM}$ and a sensitivity of $137.4 \mu \mathrm{AmM}^{-1} \mathrm{~cm}^{-2}$. Finally, an electrode was fabricated using PtNi bimetallic nanoparticles supported by graphene. The electrode performances were a response current linear to glucose concentration up to $35 \mathrm{mM}$ and a sensitivity of $20.42 \mu \mathrm{A} \mathrm{mM} \mathrm{mm}^{-1} \mathrm{~cm}^{-2}$ [625].

There are other molecules important for their role in biological processes. Among others, cholesterol and its fatty acid esters are important because one of the main constituents of mammalian cell membranes and are precursors of extra biological materials. In addition, cholesterol is one of the blood constituents and its imbalance is at the basis of various heart diseases thus making its detection very important. As made for glucose, also the determination of the cholesterol level may be done either by enzymatic and non-enzymatic routes. In the first case concentration of cholesterol is based on the detection of the $\mathrm{H}_{2} \mathrm{O}_{2}$ level which is the product reaction of cholesterol with cholesterol oxidase (ChOx). Graphene nanocomposites are generally used to fabricate cholesterol sensors. As an example, in [626] ChOx enzyme-functionalized GO was conjugated with ferrocene molecules to enhance the electron transfer. The sensor showed a linear response ranging from 0.5 to $46.5 \mathrm{mM}$ with a sensitivity of $5.71 \mathrm{~mA} \mathrm{mM}{ }^{-1} \mathrm{~cm}^{-2}$ and a LOD of $0.1 \mathrm{mM}$. A higher sensitivity of $1 \mu \mathrm{M}$ was obtained using a graphene/poly(vinylpyrrolidone)/poly(aniline) nanocomposite to fabricate the cholesterol sensing electrode [627]. The sensor showed a linear behavior in the range from $50 \mathrm{mM}$ to $10 \mathrm{mM}$. Metal nanoparticles are also used to increase the performances of the sensors. Among others, $\mathrm{Pt}$ and Pd nanoparticles are well known for their superior electrocatalytic activity. A hybrid graphene Pt decorated electrode surface was synthesized in [628] for cholesterol detection. Graphene was decorated with Pt nanoparticles by an in-situ reduction of $\mathrm{H}_{2} \mathrm{PtCl}_{6}$ in aqueous solution and functionalized with cholesterol oxidase $(\mathrm{ChOx})$ and cholesterol esterase $(\mathrm{ChEt})$. Cholesterol detection via $\mathrm{H}_{2} \mathrm{O}_{2}$ resulted in a linear response up to $12 \mathrm{mM}$ with a LOD of $0.5 \mathrm{nM}$. The same graphene/Pt hybrid material was mixed with $3 \%$ ethanolic nafion solution and drop-casted on a CGE. Two types of sensors were developed: (i) a biosensor for free cholesterol using the enzyme ChOx and (ii) a biosensor for total cholesterol measurement using the enzymes ChOx and ChEt. The sensors showed a linear response up to a $35 \mu \mathrm{M}$ cholesterol concentration and a LOD of cholesterol ester of $0.2 \mu \mathrm{M}$ (see Figure 36). 


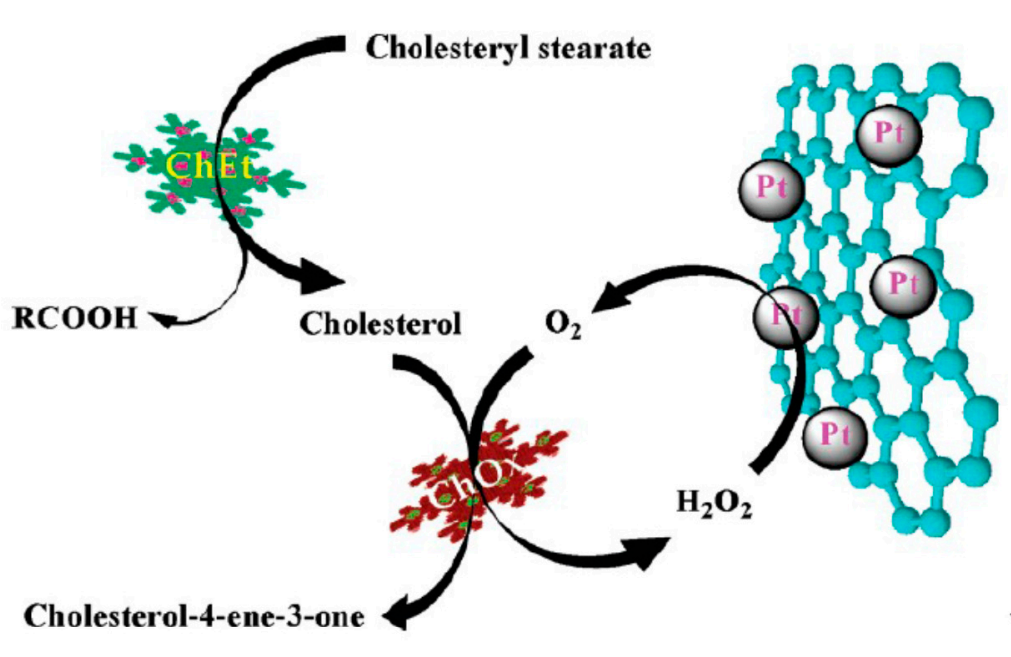

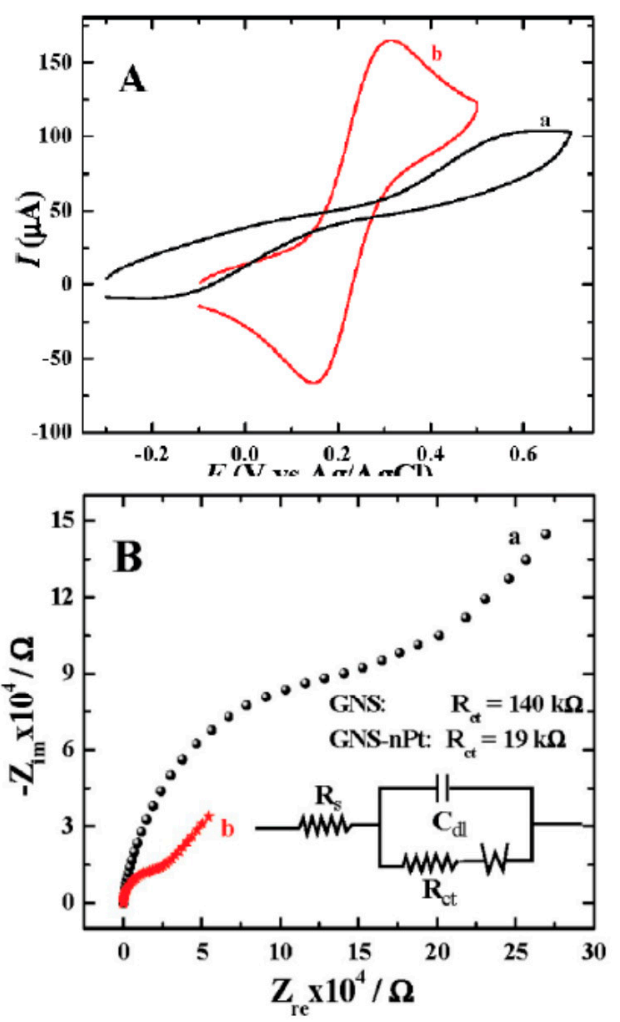

Figure 36. Left: Scheme Illustrating the Biosensing of Cholesterol Ester with the GNS-nPt-Based Biosensor. Right: Cyclic voltammetric (A) and impedance (B) response of graphene nanosheets (a) and graphene/ $\mathrm{Pt}(\mathrm{b})$ electrodes toward the redox marker $\mathrm{Fe}(\mathrm{CN})_{6}{ }^{3-}(2 \mathrm{mM})$ in $0.1 \mathrm{M}$ PBS: scan rate, $100 \mathrm{mV} / \mathrm{s}$. The inset in the impedance plot is the equivalent circuit used to fit the data. Reproduced with permission from [628]. Copyright 2010 American Chemical Society.

A cholesterol biosensor was fabricated using a rGO decorated with Pd nanoparticles [629]. The high electrocatalytic activity of dendritic Pd nanostructures led to a remarkable sensitivity of $5.12 \mathrm{~mA} \mathrm{mM}^{-1} \mathrm{~cm}^{-2}$ and a LOD of $0.05 \mathrm{mM}$. In another work a chitosan/graphene hybrid support was decorated using Pd/Pt bimetallic nanoparticles [630]. The synergistic activity of Pd and Pt catalyzers led to an accelerated direct electron transfer between $\mathrm{ChOx}$ and electrode surface. The cholesterol sensor showed a high dynamic range from $2.2 \times 10^{-6}$ to $5.2 \times 10^{-4} \mathrm{M}$, and a LOD was $0.75 \mu \mathrm{M}$. The same problems of stability affecting the GOx also influence the GhOx enzyme. To solve the problem a non-enzymatic cholesterol biosensor was fabricated using polyaniline nanofiber/graphene microflowers for the detection of cholesterol, with an impressive sensitivity of $0.101 \mathrm{~mA} \mathrm{mg}^{-1} \mathrm{dL} \mathrm{cm}^{-2}$ and an LOD of $1.93 \mathrm{mg} \mathrm{dL}^{-1}$ [631]. A different solution was adopted in [632], where authors used a nickel oxide $(\mathrm{NiO})$ /graphene composite. The sensor displayed a sensitivity

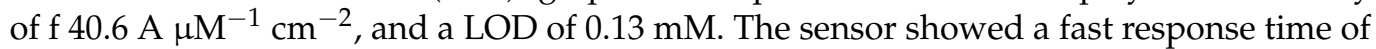
$5 \mathrm{~s}$ and a long-term stability.

Hydrogen peroxide $\left(\mathrm{H}_{2} \mathrm{O}_{2}\right)$ is another molecule important because is not only a byproduct of several enzymatic reactions but appears as a mediator in pharmaceutical, biomedical, environmental applications. This attracted great interest for developing $\mathrm{H}_{2} \mathrm{O}_{2}$ sensors although the presence of overpotentials at the working electrode likely generate a non-negligible interference [633]. $\mathrm{H}_{2} \mathrm{O}_{2}$ was detected using graphene produced via an organic salts assisted exfoliation method [634]. The sensor showed an improved electrocatalytic activity with a linear response over a range from 2.0 to $437 \mu \mathrm{M}$ and a LOD of $0.19 \mu \mathrm{M}$. The use of graphene decorated with $\mathrm{Ag}, \mathrm{Au}, \mathrm{Pt}, \mathrm{Pd}$, and $\mathrm{Cu}$ metal nanoparticles was investigated by several groups due to their catalytic properties. Pt nanoparticles were deposited on graphene sheets through a microwave-assisted heating method and utilized to detect $\mathrm{H}_{2} \mathrm{O}_{2}$ [635]. The presence of Pt nanoparticles resulted in a linear response of 
the sensor in the range 1-500 $\mathrm{mM}$ and a sensor LOD of $80 \mathrm{nM}$. Both Ag and Au possess high catalytic activity with respect to $\mathrm{H}_{2} \mathrm{O}_{2}$. Ag nanoparticles decorated graphene was synthesized by a hydrothermal procedure [636]. The Ag led to improved catalytic and conductivity of the $3 \mathrm{D}$ porous graphene structure used as sensing electrode with a linear range between $0.03-16.21 \mathrm{mM}$ and an LOD of $14.9 \mu \mathrm{M}$. In addition to nanoparticles also Ag nanorods [637] resulting in a linear sensor response in the range 0.1 to $70 \mathrm{mM}$ and a LOD of $2.04 \mu \mathrm{M}$. An alternative solution is the use of graphene/nanowires [638] hybrids leading to a sensor linearity in the range $10.0 \mu \mathrm{M}$ to $34.3 \mathrm{mM}$ and a sensitivity of $12.37 \mu \mathrm{A}$ $\mathrm{mM}^{-1} \mathrm{~cm}^{-2}$ In addition, other noble metal nanoparticles were utilized as Au [639] (linear range $0.5 \mu \mathrm{M}$ to $4.9 \mathrm{mM}$, sensitivity of $75.9 \mu \mathrm{A} \mathrm{mM}^{-1} \mathrm{~cm}^{-2}$ ), $\mathrm{Pt} / \mathrm{rGO}$ nanoparticles [640] (linear range: 1 to $900 \mu \mathrm{M}$ and a LOD of $0.1 \mu \mathrm{M}$ ), $\mathrm{Pt} / \mathrm{Ni}$ nanowires/graphene [641] (linear range: $1 \mathrm{nM}$ to $5.3 \mathrm{mM}, \mathrm{LOD}$ of $0.3 \mathrm{nM})$ or $\mathrm{Cu}_{2} \mathrm{O} /(3 \mathrm{D}, 2 \mathrm{D}) \mathrm{rGO}$ structure [642] (linear range: $1 \mu \mathrm{M}$ to $1.47 \mathrm{mM}$, were utilized for sensing $\mathrm{H}_{2} \mathrm{O}_{2}$. There are other molecules important for their role in biological processes. $\beta$-nicotinamide adenine dinucleotide $\left(\mathrm{NAD}^{+}\right)$and its reduced form (NADH) is a key factor in dehydrogenase processes which raised interest for the development of amperometric sensors. These latter are based on the redox reaction at the sensing electrode where $\mathrm{NADH}$ is oxidized in $\mathrm{NAD}^{+}$which is important for the detection of lactate, alcohol or glucose [643]. The sensing was based on a GCE modified with graphene decorated with tungstate nanoparticles. The sensor was able to detect both $\mathrm{NADH}$ and $\mathrm{NAD}^{+}$displaying a linear behavior for $\mathrm{NADH}$ and $\mathrm{NAD}^{+}$from 10-270 $\mu \mathrm{M}$ and $100-500 \mu \mathrm{M}$ and LOD equal to $2.188 \mu \mathrm{M}$ and $49.8 \mu \mathrm{M}$ for NADH and NAD ${ }^{+}$. Other biomolecules of interest are dopamine (DA) an important molecule involved in the transmission of neural signals ascorbic acid (AA) is a potent reducing and antioxidant agent and uric acid (UA) the final product of the purine metabolism and, as AA, is a potent antioxidant. Excess of UA is a symptom of altered health conditions including diabetes or kidney malfunctioning. Detection of DA, AA and UA is relevant for teranostics. A GCE modified with GO was utilized for the detection of DA in presence of interfering AA bioanalyte [644]. The sensor had a linear response in the range from $1.0 \mu \mathrm{M}$ to $15.0 \mu \mathrm{M}$ and a LOD of $0.27 \mu \mathrm{M}$ for detecting DA. In addition, no interference was observed by the addition of AA thanks to the electrostatic repulsion towards GO. Simultaneous detection of DA, AA and UA was performed using $\mathrm{Au}$ /graphene [645]. The sensor performances were: linear responses to AA, DA and UA in the ranges 30-2000, 0.5-150 and 0.5-60 $\mu \mathrm{M}$, respectively, while the LOD for AA, DA and UA are 10, 0.15 and $0.21 \mu \mathrm{M}$, respectively. DA, AA and UA can be simultaneously detected using Pt/graphene+CNTs [646] in respective concentration ranges of 200-900 mcM, 0.2-30 $\mu \mathrm{M}, 0.1-50 \mu \mathrm{M}$, showed good linearity and sensitivities of $0.186 \mu \mathrm{A} \mathrm{M}^{-1} \mathrm{~cm}^{-2}$ for AA, $9.199 \mu \mathrm{A} \mathrm{M}^{-1} \mathrm{~cm}^{-2}$ for DA and $9.386 \mu \mathrm{A} \mathrm{M}^{-1} \mathrm{~cm}^{-2}$ for UA. Other solutions to sense DA, AA and UA is the use of Ag nanowires/rGO [647] nanostructures. The sensor had a linear response of AA, DA and UA in the concentration range of 45-1550, 40-450 and 35-300 $\mu \mathrm{M}$ with a detection limit of $0.81,0.26$ and $0.30 \mu \mathrm{M}$ respectively. Decorated graphene/polymer hybrid systems are also utilized to detect DA, AA and UA. For example sensors were fabricated using Pd/graphene+chistosan [648] with peak potential separations between AA and DA, DA and UA, AA and UA were $252 \mathrm{mV}$, $144 \mathrm{mV}$ and $396 \mathrm{mV}$, respectively. In addition, the LOD $(\mathrm{S} / \mathrm{N}=3)$ for AA, DA and UA were $20 \mu \mathrm{M}, 0.1 \mu \mathrm{M}$ and $0.17 \mu \mathrm{M}$, respectively. Another possibility is $\mathrm{MoS}_{2}$ decorated PANI-rGO GCE [649]. Pulse voltammetry was utilized to characterize the sensor obtaining linear responses in a wide range from 5.0 to $500 \mu \mathrm{M}, 50 \mu \mathrm{M}$ to $8.0 \mathrm{mM}$, and 1.0 to $500 \mu \mathrm{M}$, giving low detection limits of $0.70,22.20$, and $0.36 \mu \mathrm{M}$ for DA, AA, and UA, respectively. Differential pulse voltammetry applied to $\mathrm{ZnO}$ nanowire arrays/Graphene foam modified electrodes was used to obtain higher sensitivity, leading to a LOD of $0.5,5$, and $0.5 \mu \mathrm{M}$ for DA, AA and UA when detected simultaneously [650]. $\mathrm{SnO}_{2}-\mathrm{rGO}$ electrode was utilized to detect urea [651]. Amperometry show that the $\mathrm{SnO}_{2}-\mathrm{rGO} / \mathrm{GCE}$ electrode is sensitive to urea in the concentration range of 0.016 to $3.9 \mathrm{pM}$, with a LOD as low as $11.7 \mathrm{fM}$.

Graphene is also used as material of choice for the detection of genetic substances important for the recognition of specific pathologies. DNA sensors can be based on differ- 
ent transduction techniques as electrochemistry electrochemiluminescence, fluorescence, surface plasmon resonance, colorimetry [652]. The electrochemical sensors possess a high sensitivity, are fast and low cost. The electrochemical detection mechanism is based on the change of the sensor response upon DNA sequence hybridization. Owing to their unique molecular structure and electronic properties graphene and its derivatives have captured much attention for the electrochemical sensing of genetic matter. In particular, it has been demonstrated that DNA or RNA interact with graphene with weak $\pi-\pi$ interactions [653]. This allows tailoring the electronic properties of the graphene adsorbate thus optimizing the support effective signal amplification. Au NP decorated rGO was utilized to assemble an electrochemical sensor. This latter showed good selectivity towards single base DNA sequences, a linear behavior in the range $0.1 \mathrm{fM}$ to $0.1 \mu \mathrm{M}$ and a LOD of $35 \mathrm{aM}$ [654]. Graphene/Au nanorods/polythionine was utilized to produce a biosensor for the detection of human papillomavirus [655]. The device showed unique sensing properties being able to detect the DNA papillomavirus in a range $1 \times 10^{-13}$ to $1 \times 10^{-10} \mathrm{~mol} / \mathrm{L}$ with a LOD of $4.03 \times 10^{-14} \mathrm{~mol} / \mathrm{L}$. In another work, graphene bifunctionalized with riboflavin 50monophosphate sodium salt (FMNS) leading to high stability and sensitivity with a LOD of $8.3 \times 10^{-17} \mathrm{M}$ slightly higher than $7.4 \times 10^{-17} \mathrm{M}$ that of the conventional electrochemical indicator $\left(\left[\mathrm{Fe}(\mathrm{CN})_{6}\right]^{3-/ 4-}\right)$ [656]. Other researchers developed a GO/chitosan composite DNA sensor for the detection of the typhoid infection [657]. At this aim, a Salmonella single strand DNA was immobilized on the GO/Chitosan-ITO electrode using a glutaraldehyde crosslinker. The use of GO/Chitosan hybrid composite led to high sensing efficiency due to the fast electron transfer from the DNA and the ITO substrate. The biosensor was selective in the recognition of the complementary or non-complementary DNA sequences differing for only one base. The detection was linear in the range from $10 \mathrm{fM}$ to $50 \mathrm{nM}$ with a LOD of $10 \mathrm{fM}$ within a response times of $60 \mathrm{~s}$ required for hybridization of the complementary sequence [657]. In addition, the detection may be performed in serum samples with a low LOD of $100 \mathrm{fM}$. The sensing activity may be reinforced by the integration of metallic $\mathrm{NP}$ in the device. The detection of a gene for the multidrug resistance was made using AuNPs/toluidine blue/GO electrode (see Figure 37) [658]. Under optimal conditions, the amperometric biosensor measured a current proportional to the logarithm of the target DNA concentration in the range from $1.0 \times 10^{-11}$ to $1.0 \times 10^{-9} \mathrm{M}$ with a detection limit of $2.95 \times 10^{-12} \mathrm{M}$. Moreover, the biosensor showed good selectivity and a reasonable stability and reproducibility.

In other sensors were utilized gold nanorods supported on GO as reported in [659]. The biosensor showed high selectivity being able to detect the complementary DNA sequence from a mixture with a 1000:1 proportion. In addition, the sensor had a linear behavior in a $1.0 \mathrm{nM}$ to $10 \mathrm{fM}$ with a LOD of $3.5 \mathrm{fM}$.

A list of different methods has been proposed to amplify the DNA detection signal. For example, in [660] is described the fabrication of a sensor for the detection of the human immunodeficiency virus (HIV) gene. Authors utilized an Au decorated graphene electrode using gold nanoclusters and applying an exonuclease III-assisted target recycling amplification method shown in Figure 38 obtaining a sensor with LOD of $30 \mathrm{aM}$.

Besides amplification, the biosensor showed high selectivity towards interfering sequences at concentrations 10 times higher than the HIV target. Recently an opposite route is gaining increasing interest: biosensensing activity is based on enhanced sensitivity obtained with the use of CRISPR-Cas9 to instead of amplification of the target DNA [661]. In conventional graphene FET based on complementary probe DNA, the sensitivity is limited by the concentration of the target sequences on the sensing electrode. An alternative method recently utilized, consists in a graphene-based field-effect transistor (FET) that uses clusters a matrix of short palindromic repeats (CRISPR) to enable the digital detection of a target DNA sequence. Without the need for amplification, the CRISP based technology in $15 \mathrm{~min}$ is able to generate a detection signal with a sensitivity of $1.7 \mathrm{fM}$. The graphene electrode is functionalized with a deactivated Cas9 CRISP (denoted with dRNP) complex which scans the whole genomic sample unzipping the double helix until finds the target 
sequence. Now Cas9 binds to the target sequence modulating the FET output. Two kinds of DNA were used, the first collected from HEK293T cell lines expressing blue fluorescent protein, the second derived from clinical samples and showing two distinct mutations at exons commonly deleted in individuals with Duchenne muscular dystrophy. The CRISP chip functionalized with the mutating Duchenne sequence produced an enhanced signal output only in presence of the complementary target.

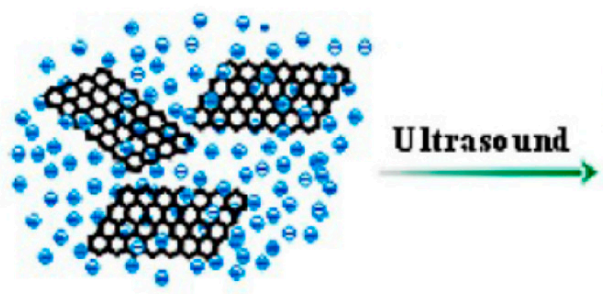

(A)

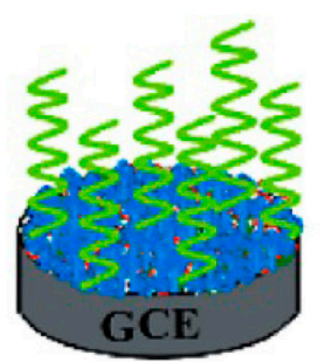

(F)

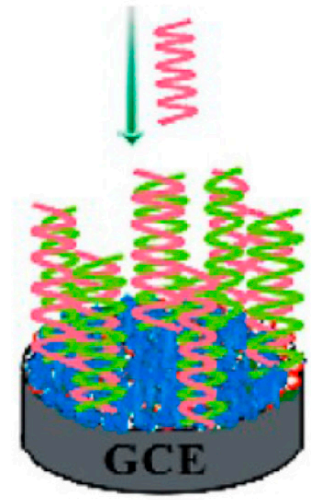

(G)

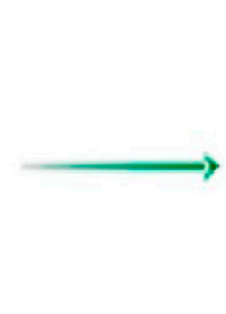

(1)

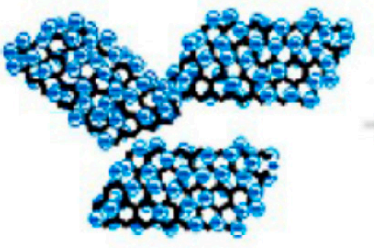

(B)

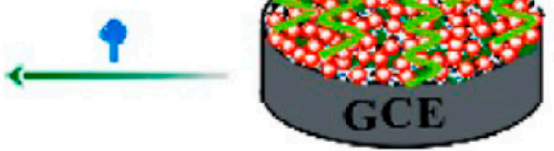

(E)

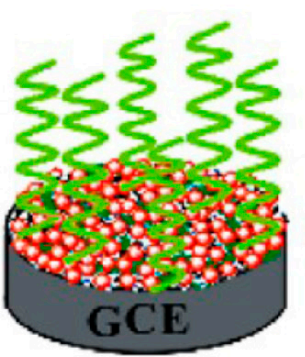

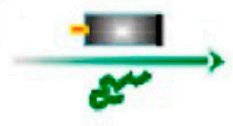

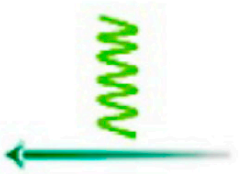

GO

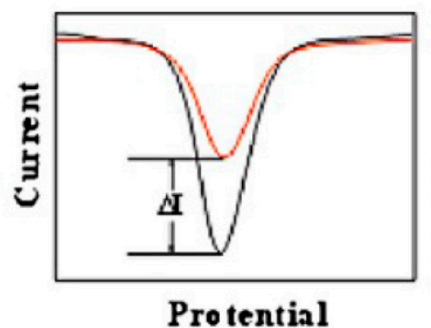

(H)

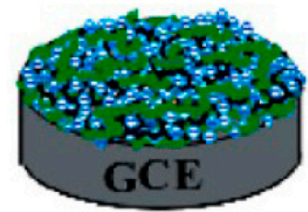

(C)

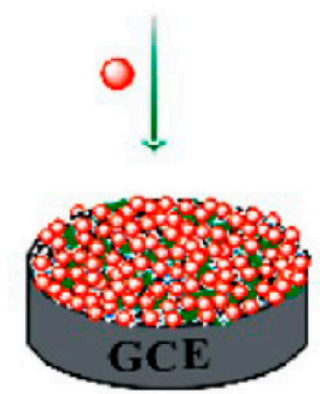

(D)

Figure 37. (A,B) coupling toluidine blue to GO; (C) deposition of the functionalized GO to a GCE; (D) linking Au NP to the electrode; (E) adsorption of the AuNP to the GO/toluidine/GCE; thiol terminated single strand DNA probes where covalently bonded to the Au NPs; (F) the electrode stabilized with mercapto-1-hexanol is ready for DNA detection; (G) hybridization of the probe and target DNA sequences; $(\mathbf{H})$ amperometric detection of the target DNA. Reprinted with permission from [658]. Copyright 2015 Elsevier. 


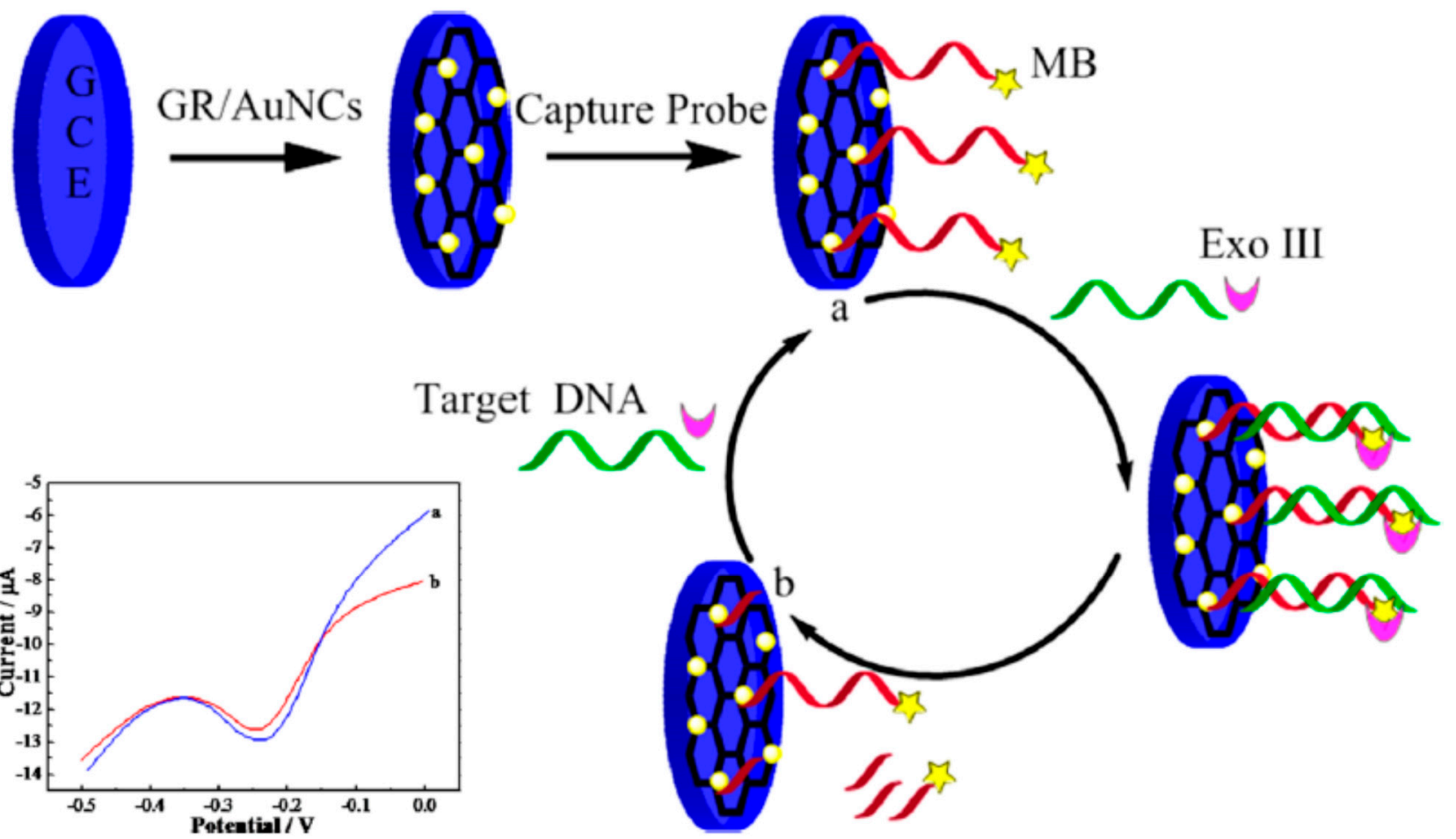

Figure 38. Au nanocluster decorated graphene is deposited on a GCE. Then a single strand capture probe is attached to the $\mathrm{Au}$ nanoclusters leading to an output current signal (a). In presence of the target DNA hybridization occurs and a variation of the current at the sensor electrode is obtained (b). The presence of Exo III leads to the digestion of the capture probe with release of the target DNA which is then ready for further hybridization reactions. In this way, an ultrasensitive signal-off electrical biosensor was realized. Reproduced with permission from [660]. Copyright 2015 American Chemical Society.

In [662] the device sensitivity is amplified by a factor of 20,000 using a hairpin probe DNA allowing a recycling and hybridization of the target. The sensor was capable to sense a 21 mer target DNA at sub-fM concentration with superior selectivity towards mismatched oligomers. In this sensor the sensitivity is independent on the binding affinity of nucleic acids as in conventional sensors.

Besides genomic mutations, cancer is a disease with high socio-economical impact. Detection of cancer markers is then of high importance for the prevention, early detection, the diagnosis and the care. Pd decorated rGO electrochemical sensor were fabricated for a label free detection of the prostate specific antigen, a biomarker of the prostate cancer [663]. The biosensor showed a good sensitivity of $28.96 \mathrm{~mA} \mathrm{~mL} \mathrm{ng}{ }^{-1} \mathrm{~cm}^{-2}$ with an LOD of $10 \mathrm{pg} \mathrm{mL}^{-1}$. Other metallic nanoparticles as $\mathrm{Pd}, \mathrm{Pt}, \mathrm{Ag}$ and $\mathrm{Au}$ were deposited on rGO sheets to fabricate a nanocomposite-based sensor for the detection of epidermal growth factor receptor ErbB2 useful for the breast cancer diagnosis [664] with a LOD varying in the range $1.0 \mathrm{fM}$ to $0.5 \mu \mathrm{M}$ upon the different metal nanoparticle used.

Reduced graphene oxide sheets were decorated with iron nitride nanoparticles $\left(\mathrm{Fe}_{2} \mathrm{~N}\right)$ NPs produced by a solvothermal method and followed by a nitridation process [665]. The nanocomposite was screen printed to fabricate electrodes possessing high electrochemical sensing properties toward 4-nitroquinoline $\mathrm{N}$-oxide (4-NQO) an important cancer marker. The sensor showed a linear response in the range $0.05-574.2 \mu \mathrm{M}$ and nanomolar detection limit $(9.24 \mathrm{nM})$. Finally, the biosensor was utilized to analyze human blood and urine samples showing recoveries close to $100 \%$. In another work, an emulsion of GO and watertoluene were self-assembled in microspheres were used to fabricate an electrochemical sensor [666]. The GO microspheres were functionalized with folic acid and octadecylamine and then dried at $400{ }^{\circ} \mathrm{C}$ for $5 \mathrm{~h}$. The microspheres of $\sim 1.2 \mu \mathrm{m}$ size are characterized by a porous structure densely functionalized with ordered alignment of folic acid groups allowing a high efficiency in capturing specific cancer cell. The sensor has a linear behavior 
in range of $5-10^{5}$ cell $/ \mathrm{mL}$ with a LOD of 5 cells $\mathrm{mL}^{-1}$. The biosensor was able to selectively discriminate between HepG2, HeLa and A549 model cancer cell lines and L02 cell line used as a control. The efficiency in the selective detection of cancer cells was attributed to the overexpression of folic acid on the cancer cell membrane. Another recent example is a cancer biomarker detection based on sulfur-doped reduced graphene oxide ( $\mathrm{SrGO}$ ) product fabricated using an eco-friendly biomass precursor [667]. The functionalized graphene is used to assemble a highly sensitive electrochemical sensor for detecting of 8-hydroxy-2'-deoxyguanosine (8-OHdG) molecules, which are an important biomarker for oxidative stress, cardiovascular diseases, and cancers. The biomarker displayed an excellent electrochemical sensitivity attributed to the strong electron-donating ability of sulfur facilitating the electron transfer to the biomolecules in the redox reactions. The device possesses a sensitivity of $\sim 1 \mathrm{nM}$, a $20-0.002 \mu \mathrm{M}$ wide detection window, good selectivity, stability and reproducibility (see Figure 39). Finally, in optimized experimental conditions, the biosensor showed remarkable recoveries for the detection of 8-OHdG biomarker.

(1)

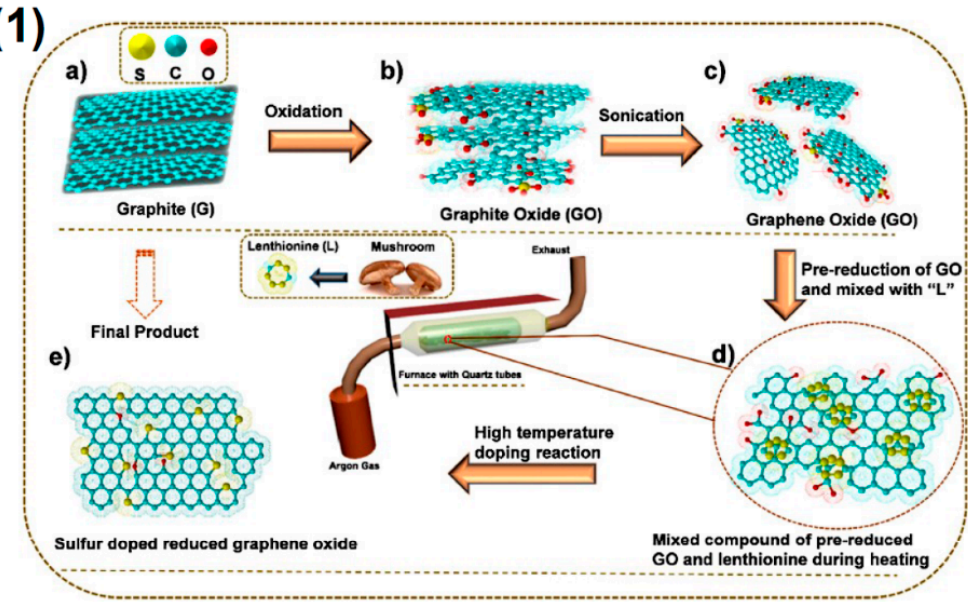

(2)

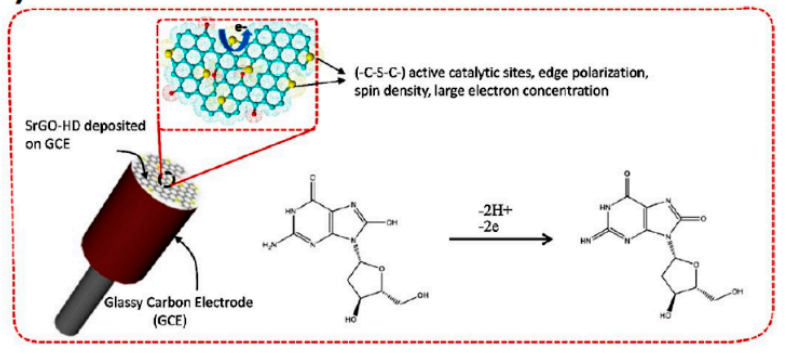

(3)

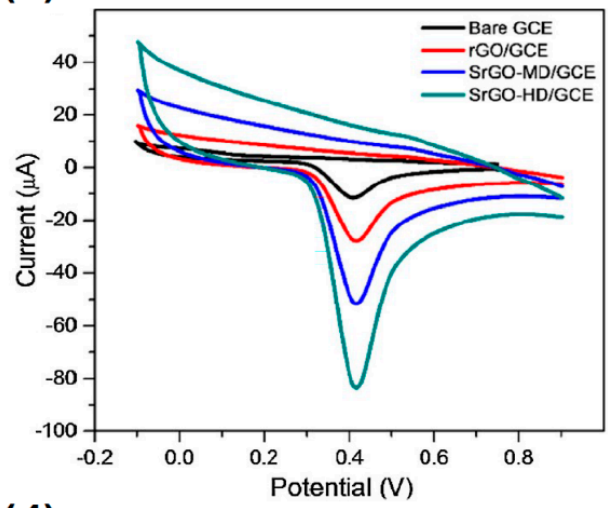

(4)

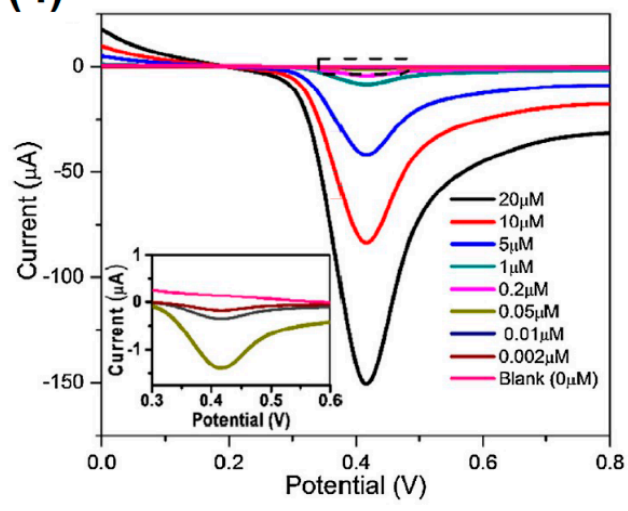

Figure 39. (1) Synthesis of S-doped reduced graphene oxide: (a) Graphite; (b) Oxidation of graphite to get graphite oxide (GO); (c) Sonication of GO to get exfoliated graphene oxide; (d) mixing of pre-reduced graphene oxide with lenthionine; (e) final product after high-temperature thermal treatment to obtain S-doped reduced graphene oxide; (2) The schematic representation of GCE modification and mechanism of electrochemical sensor for 8-OHdG by sulfur-doped reduced graphene oxide. (3) Cyclic voltammograms of bare GCE, rGO/GCE, SrGO-MD/GCE, and SrGO-HD/GCE in the presence of $10 \mu \mathrm{M}$ 8-OHdG in0.1 M PBS (pH 7.2) at scan rate of $100 \mathrm{mV} / \mathrm{s}$. (4) Biosensor responses at different concentrations for 8-OHdG with SrGO-HD/GCE sensor (inset shows the dotted rectangle zoom-in of lower concentrations) in 0.1 MPBS (pH 7.2) at scan rate of $100 \mathrm{mV} / \mathrm{s}$;. Reproduced with permission from [667]. Copyright 2017 Elsevier.

Decoration of graphene with metal NPs is another effective route to improve the detection of cancer biomarkers. Among others, several studies have been carried out to study the graphene/AuNPs electrochemical response for various cancer biomarkers. In such work a three-dimensional graphene/gold NP composite was fabricated for a selective detection of the cytokeratin 19 fragment antigen 21-1 (CYFRA 21-1) [668]. The label-free electrochemical 
immunosensor was capable to selectively detect the tumor marker CYFRA21-1 in non-small cell lung cancer. Briefly, the anti-CYFRA21-1 was immobilized on the Graphene/AuNP modified GCE through the cross-linking of chitosan (CS), glutaraldehyde (GA).

The electrochemical immunosensor was employed to detect CYFRA21-1 and exhibited a wide linear range of $0.25-800 \mathrm{ng} / \mathrm{mL}$ and low detection limit of $100 \mathrm{pg} / \mathrm{mL}$. Other nanoparticles as cobalt sulfide [669] (detection of CA15-3 antigen in wide linear range of 0.1-150 $\mathrm{U} \mathrm{mL}^{-1}$ and a low detection limit of $0.03 \mathrm{U} \mathrm{mL}^{-1}$ ), $\mathrm{Pd}$ [670] (prostate-specific antigen with sensitivity $28.96 \mu \mathrm{A} \mathrm{mL} \mathrm{ng}{ }^{-1} \mathrm{~cm}^{-2}$ corresponding to $10 \mathrm{pg} \mathrm{mL}^{-1}$ ), oxides as $\mathrm{ZrO}_{2}$ [671] (CYFRA-21-1 biomarker with sensitivity $0.756 \mu \mathrm{A} \mathrm{mL} \mathrm{ng}{ }^{-1}$ and a remarkable lower detection limit of $0.122 \mathrm{ng} \mathrm{mL}^{-1}$ ), $\mathrm{Fe}_{3} \mathrm{O}_{4}$ [672] (detection of prostate specific antigen or prostate specific membrane antigen with excellent LOD of $15 \mathrm{fg} / \mathrm{mL}$ for PSA and $4.8 \mathrm{fg} / \mathrm{mL}$ for PSMA were achieved in serum).

A few words must be spent for describing the fabrication of sensors for heavy metal ions which are known to be dangerous for the human health. Electrochemical sensors based on graphene and graphene-based materials are characterized by high sensitivity and selectivity and high stability, rapid response time and low cost thus resulting useful for heavy metal ions detection. There are several review papers dedicated to the description of graphene-based nanomaterials for the detection of some important heavy metal ions [673-675]. In [676] are reviewed graphene-based sensors based on $\mathrm{Cu}^{2+}, \mathrm{Pb}^{2+}, \mathrm{Hg}^{2+}$, $\mathrm{Cd}^{2+} ; \mathrm{Ag}^{2+}$. The use of graphene-based nanomaterials resulted in an improvement of the performances of heavy metal ion sensors. From a survey of the literature the heavy metal ions are detected using graphene based sensors and different electrochemical techniques as differential pulse voltammetry (DPV), differential pulse stripping voltammetry (DPSV), differential pulse anodic stripping voltammetry (DPASV), square wave anodic stripping voltammetry (SWASV) obtaining different degrees of detection limits [673]. In the case of $\mathrm{Cd}(\mathrm{II})$ the LOD ranged from $0.01 \mu \mathrm{g} / \mathrm{L}$ to $0.6 \mu \mathrm{g} / \mathrm{L}$ using DPV, DPASV or SWASV, while in other works and using different concentration units, LOD ranged from $0.19 \mathrm{nM}$ to $3 \mathrm{nM}$. $\mathrm{Pb}$ (II) is detected using DPV, DPASV, SWASV obtaining LOD ranging from $0.02 \mu \mathrm{g} / \mathrm{L}$ to $0.41 \mu \mathrm{g} / \mathrm{L}$, other authors found a LOD ranging from $0.6 \mathrm{nM}$ to $1.41 \mathrm{nM}$ using DPV, SWASV. As(III) was detected using DPV obtaining a LOD from $2.3 \mathrm{nM}$. Hg(II) was detected using DPASV resulting in a LOD of $0.277 \mathrm{nM}$. Cu(II) ions were detected using DPASV, SWASV, with LOD in the range $0.16 \mathrm{nM}-0.52 \mathrm{nM}$ or LOD $=0.02 \mu \mathrm{g} / \mathrm{L} . \mathrm{Zn}$ (II) ions were detected using DPASV and SWASV obtaininf a LOD in the range $0.1-0.18 \mu \mathrm{g} / \mathrm{L}$. Data regarding the sensitivities reached using the various techniques are summarized in the Table 6, while more information may be found in the given references.

Toxic substances as pesticides, herbicides and insecticides used to protect plants in agriculture as well as those produced in industrial processes and toxic gases developed by human activities need a systematic control for societal safety.

Graphene based sensors offer an option for the sensitive detection of agro-hazardous pesticide residues in food [677-680], and to control the quality of the water and air to avoid dangerous effects to humans. Electrochemical sensors play also a significant role in the industry and in everyday life to control toxic substances. For example, controlling the presence of 4-nitrochlorobenzene a toxic organic pollutant is highly desirable. Graphene based electrochemical sensors are the low cost, highly sensitive and offer ease of operation for the detection of toxix substances as: 4-nitrochlorobenzene [681] (LOD = $10 \mathrm{nM}$ ), for the detection of hydrazine [682], sulfides [683], 4-aminophenol [684], diphenolic compounds [685], hydroquinone [686]. In addition, toxic gaseous species can be detected as reported in [687] and example are $\mathrm{H}_{2}$ [688] (LOD: 20 ppm), $\mathrm{CO}$ [689] (LOD: 0.25 ppm), $\mathrm{CO}_{2}$ [690] (sensitivity 0.17\%/ppm), NO [691] (sensitivity: 63.65 pm/ppm), NO $\mathrm{NO}_{2}$ [692] (LOD: 0.5 ppm) $\mathrm{SO}_{2}$ [693] (LOD: $0.5 \mathrm{ppm}$ ), ammonia [694,695]. More information may be found in the review on the toxic gas detection based on graphene metal-oxides [696]. 
Table 6. Detection modality, analyte and detection limit of electrochemical graphene-based sensors.

\begin{tabular}{|c|c|c|c|c|}
\hline Method & Functionalization & Analyte/Entity & Performances & Reference \\
\hline Optical & Pristine Graphene & Young Modulus & $1.9 \pm 0.4 \mathrm{~cm}^{-1 /} \mathrm{GPa}$ & {$[604]$} \\
\hline Optical & Pristine Graphene & Young Modulus & $\begin{array}{l}\partial \omega_{G+} / \partial \varepsilon \sim-18.6 \mathrm{~cm}^{-1} / \% \\
\partial \omega_{G-} / \partial \varepsilon \sim-36.4 \mathrm{~cm}^{-1} / \%\end{array}$ & {$[605]$} \\
\hline Piezo-Resistivity & Pristine Graphene & Strain & $\begin{array}{c}\mathrm{GF}^{1}=\text { from } 4 \text { to } 14 \text { for } \\
\text { strains }>1.8 \%\end{array}$ & {$[608]$} \\
\hline Piezo-Resistivity & Reduced Graphene & Strain & $\mathrm{GF}=9.49$ & [609] \\
\hline Piezo-Resistivity & Reduced Graphene & Strain & Sensitivity of $0.18 \mathrm{kPa}^{-1}$ & [610] \\
\hline Amperometric & $\mathrm{GOx}-\mathrm{Au} / \mathrm{GO}$ & Glucose & $\begin{array}{l}\text { Sensitivity of } 42 \mathrm{~mA} \\
\mathrm{mM}^{-1} \mathrm{~cm}^{-2}\end{array}$ & {$[614]$} \\
\hline Amperometric & $\mathrm{NiCo} 2 \mathrm{~N} / \mathrm{GO}$ & $\begin{array}{c}\text { Glucose } \\
\mathrm{H}_{2} \mathrm{O}_{2}\end{array}$ & $\begin{array}{l}\text { LOD: } 50 \mathrm{nM} \text { for glucose } \\
200 \mathrm{nM} \text { for } \mathrm{H}_{2} \mathrm{O}_{2}\end{array}$ & [622] \\
\hline Amperometric & $\mathrm{Cu} / \mathrm{GO}$ & Glucose & $48.13 \mu \mathrm{A} \mathrm{mM}^{-1}$ & {$[623]$} \\
\hline Amperometric & $\mathrm{Pt} / \mathrm{GO}$ & Glucose & $\begin{array}{l}\text { Sensitivity ofSensitivity } \\
137.4 \mu \mathrm{A} \mathrm{mM} \mathrm{mM}^{-1} \mathrm{~cm}^{-2}\end{array}$ & {$[624]$} \\
\hline Amperometric & $\mathrm{PtNi} / \mathrm{GO}$ & Glucose & $\begin{array}{c}\text { Sensitivity } 20.42 \mu \mathrm{A} \\
\mathrm{mM}^{-1} \mathrm{~cm}^{-2}\end{array}$ & {$[625]$} \\
\hline Amperometric & ChOx-ferrocene/GO & Cholesterol & $0.1 \mathrm{mM}$ & {$[626]$} \\
\hline Amperometric & poly(vinylpyrrolidone)/poly(aniline)/graphene & Cholesterol & $1 \mu \mathrm{M}$ & {$[627]$} \\
\hline Amperometric & $\mathrm{ChOx}, \mathrm{ChEt} / \mathrm{Pt} /$ graphene & $\begin{array}{c}\text { Cholesterol via } \mathrm{H}_{2} \mathrm{O}_{2} \\
\text { Cholesterol direct }\end{array}$ & $\begin{array}{c}0.5 \mathrm{nM}\left(\mathrm{H}_{2} \mathrm{O}_{2}\right) \\
0.2 \mu \mathrm{M} \text { (Cholesterol) }\end{array}$ & {$[628]$} \\
\hline Amperometric & Dendritic Pd/rGO structure & Cholesterol & $0.05 \mathrm{mM}$ & {$[629]$} \\
\hline Amperometric & Pt-Pd chitosan/Graphene & Cholesterol & LOD: $0.75 \mu \mathrm{M}$ & [630] \\
\hline Amperometric & polyaniline nanofiber/graphene & Cholesterol & LOD: $1.93 \mathrm{mg} \mathrm{dL}^{-1}$ & [631] \\
\hline Amperometric & $\mathrm{NiO} /$ graphene & Cholesterol & LOD: $0.13 \mathrm{mM}$ & [632] \\
\hline Amperometric & Graphene & $\mathrm{H}_{2} \mathrm{O}_{2}$ & LOD: $0.19 \mu \mathrm{M}$ & [634] \\
\hline Amperometric & Pt/Graphene & $\mathrm{H}_{2} \mathrm{O}_{2}$ & LOD: $80 \mathrm{nM}$ & [635] \\
\hline Amperometric & Ag/Graphene & $\mathrm{H}_{2} \mathrm{O}_{2}$ & LOD: $14.9 \mu \mathrm{M}$ & {$[636]$} \\
\hline Amperometric & $\mathrm{Ag}$ rods $/ \mathrm{rGO}$ & $\mathrm{H}_{2} \mathrm{O}_{2}$ & LOD: $2.04 \mu \mathrm{M}$ & [637] \\
\hline Amperometric & Ag wires/Graphene & $\mathrm{H}_{2} \mathrm{O}_{2}$ & $\begin{array}{c}\text { Sensitivity of } 12.37 \mu \mathrm{A} \\
\mathrm{mM}^{-1} \mathrm{~cm}^{-2}\end{array}$ & {$[638]$} \\
\hline Amperometric & Au/Graphene & $\mathrm{H}_{2} \mathrm{O}_{2}$ & $\begin{array}{c}\text { Sensitivity of } 75.9 \mu \mathrm{A} \\
\mathrm{mM}^{-1} \mathrm{~cm}^{-2}\end{array}$ & {$[639]$} \\
\hline Amperometric & P/Graphene & $\mathrm{H}_{2} \mathrm{O}_{2}$ & LOD: $0.1 \mu \mathrm{M}$ & [640] \\
\hline Amperometric & PtNi nanowires/Graphene & $\mathrm{H}_{2} \mathrm{O}_{2}$ & LOD: $0.31 \mathrm{nM}$ & [641] \\
\hline Amperometric & $\mathrm{Cu}_{2} \mathrm{O} /(3 \mathrm{D}, 2 \mathrm{D}) \mathrm{rGO}$ structure & $\mathrm{H}_{2} \mathrm{O}_{2}$ & LOD: $0.37 \mu \mathrm{M}$ & [642] \\
\hline Amperometric & $\mathrm{WO}_{4}-2 / \mathrm{G}$ & NADH, NAD ${ }^{+}$ & $\begin{array}{l}\text { LOD: } 2.188 \mu \mathrm{M} \text { for } \mathrm{NADH} \\
\text { and } 49.8 \mu \mathrm{M} \text { for } \mathrm{NAD}^{+}\end{array}$ & {$[643]$} \\
\hline Chronoamperometry & $\mathrm{GO}$ & Dopamine & LOD: $0.27 \mu \mathrm{M}$ & {$[644]$} \\
\hline $\begin{array}{c}\text { Square } \\
\text { voltammetry }\end{array}$ & AU/Graphene & $\begin{array}{l}\text { Dopamine, } \\
\text { Ascorbic acid, } \\
\text { Uric acid }\end{array}$ & $\begin{array}{c}\text { LOD: } 10 \mu \mathrm{M} \text { for } \mathrm{DA}, 0.15 \\
\mu \mathrm{M} \text { for } \mathrm{AA} \text {, and } 0.21 \mu \mathrm{M} \text { for } \\
\mathrm{UA}\end{array}$ & - \\
\hline Amperometric & Pt/Graphene & $\begin{array}{l}\text { Dopamine, } \\
\text { Ascorbic acid, } \\
\text { Uric acid }\end{array}$ & $\begin{array}{l}\text { Sensitivity: } 0.186 \mu \mathrm{A} \mathrm{M}^{-1} \\
\mathrm{~cm}^{-2} \text { for AA, } 9.199 \mu \mathrm{A} \mathrm{M}^{-1} \\
\mathrm{~cm}^{-2} \text { for DA and } 9.386 \mu \mathrm{A} \\
\mathrm{M}^{-1} \mathrm{~cm}^{-2} \text { for UA. }\end{array}$ & [646] \\
\hline Voltammetry & $\mathrm{Ag} / \mathrm{rGO}$ & $\begin{array}{l}\text { Dopamine, } \\
\text { Ascorbic acid, } \\
\text { Uric acid }\end{array}$ & $\begin{array}{c}\text { LOD: } 0.81 \mu \mathrm{M} \text { for } \mathrm{DA}, 0.26 \\
\mu \mathrm{M} \text { for AA and } 0.30 \mu \mathrm{M} \text { for } \\
\mathrm{UA}\end{array}$ & [647] \\
\hline
\end{tabular}


Table 6. Cont.

\begin{tabular}{|c|c|c|c|c|}
\hline Method & Functionalization & Analyte/Entity & Performances & Reference \\
\hline Voltammetry & Pd-chitosan/Graphene & $\begin{array}{l}\text { Dopamine, } \\
\text { Ascorbic acid, } \\
\text { Uric acid }\end{array}$ & $\begin{array}{l}\text { LOD: } 0.1 \mu \mathrm{M} \text { for DA, } 20 \mu \mathrm{M} \\
\text { for AA and } 0.17 \mu \mathrm{M} \text { for UA }\end{array}$ & [648] \\
\hline Pulse voltammetry & $\mathrm{MoS}_{2}$ decorated PANI/rGO & $\begin{array}{l}\text { Dopamine, } \\
\text { Ascorbic acid, } \\
\text { Uric acid }\end{array}$ & $\begin{array}{l}\text { LOD: } 0.7 \mu \mathrm{M} \text { for DA, } 22.2 \\
\mu \mathrm{M} \text { for AA and } 0.36 \mu \mathrm{M} \text { for } \\
\mathrm{UA}\end{array}$ & [649] \\
\hline $\begin{array}{l}\text { Differential pulse } \\
\text { voltammetry }\end{array}$ & $\mathrm{ZnO}$ nanowire array/Graphene foam & $\begin{array}{l}\text { Dopamine, } \\
\text { Ascorbic acid, } \\
\text { Uric acid }\end{array}$ & $\begin{array}{l}\text { LOD: } 0.5 \mu \mathrm{M} \text { for } \mathrm{DA}, 5 \mu \mathrm{M} \\
\text { for AA and } 0.5 \mu \mathrm{M} \text { for } \mathrm{UA}\end{array}$ & {$[650]$} \\
\hline Amperometry & $\mathrm{SnO}_{2} / \mathrm{rGO}$ & Urea & LOD: $11.7 \mathrm{fM}$ & {$[651]$} \\
\hline $\begin{array}{l}\text { Differential pulse } \\
\text { voltammetry }\end{array}$ & $\mathrm{Au} / \mathrm{rGO}$ & DNA & LOD: 35 aM & {$[654]$} \\
\hline $\begin{array}{c}\text { Impedance } \\
\text { spectroscopy }\end{array}$ & nanorods/polythionine-Au/Graphene & DNA & LOD: $4.03 \times 10^{-14} \mathrm{~mol} / \mathrm{L}$ & {$[655]$} \\
\hline $\begin{array}{l}\text { Differential pulse } \\
\text { voltammetry }\end{array}$ & riboflavin 50-monophosphate $\mathrm{Na}$ /graphene & DNA & LOD: $8.3 \times 10^{-17} \mathrm{M}$ & {$[656]$} \\
\hline $\begin{array}{l}\text { Differential pulse } \\
\text { voltammetry }\end{array}$ & Chitosan/GO & DNA & LOD: $10 \mathrm{fM}$ & {$[657]$} \\
\hline Amperometry & AuNPs-toluidine blue/GO & DNA & LOD: $2.95 \times 10^{-12} \mathrm{M}$ & {$[658]$} \\
\hline $\begin{array}{l}\text { Differential pulse } \\
\text { voltammetry }\end{array}$ & Au nanorods/GO & DNA & LOD: $3.5 \mathrm{fM}$ & {$[659]$} \\
\hline $\begin{array}{l}\text { Differential pulse } \\
\text { voltammetry }\end{array}$ & Au/Graphene & DNA & LOD: $30 \mathrm{aM}$ & {$[660]$} \\
\hline Amperometry & Graphene FET $^{1}$ & DNA & LOD: $1.7 \mathrm{fM}$ & {$[661]$} \\
\hline Amperometry & Graphene FET $^{1}$ & DNA & LOD: $<1 \mathrm{fM}$ & {$[662]$} \\
\hline Voltammetry & $\mathrm{Pd} / \mathrm{rGO}$ & $\begin{array}{l}\text { carcinoembryonic } \\
\text { antigen }\end{array}$ & LOD: $10 \mathrm{pg} \mathrm{mL}{ }^{-1}$ & {$[663]$} \\
\hline Resistivity & $\mathrm{Pd}, \mathrm{Pt}, \mathrm{Ag}$ and $\mathrm{Au} / \mathrm{rGO}$ & ErbB2 & LOD: $1.0 \mathrm{fM}$ to $0.5 \mu \mathrm{M}$ & {$[664]$} \\
\hline $\begin{array}{l}\text { Differential pulse } \\
\text { voltammetry }\end{array}$ & $\mathrm{Fe}_{2} \mathrm{~N} / \mathrm{rGO}$ & $\begin{array}{l}\text { 4-nitroquinoline } \\
\text { N-oxide }\end{array}$ & LOD: $9.24 \mathrm{nM}$ & {$[665]$} \\
\hline $\begin{array}{l}\text { Differential pulse } \\
\text { voltammetry }\end{array}$ & folic acid-octadecylamine/GO & HepG2 cells & LOD: 5 cells $\mathrm{mL}^{-1}$ & {$[666]$} \\
\hline $\begin{array}{l}\text { Differential pulse } \\
\text { voltammetry }\end{array}$ & S-doped rGO & $\begin{array}{c}\text { 8-hydroxy-2'- } \\
\text { deoxyguanosine } \\
\text { (8-OHdG) molecules }\end{array}$ & LOD: $\sim 1 \mathrm{nM}$ & {$[667]$} \\
\hline $\begin{array}{l}\text { Differential pulse } \\
\text { voltammetry }\end{array}$ & Au/Graphene & $\begin{array}{l}\text { cytokeratin } 19 \\
\text { fragment antigen 21-1 }\end{array}$ & LOD: $100 \mathrm{pg} / \mathrm{mL}$ & {$[668]$} \\
\hline $\begin{array}{l}\text { Differential pulse } \\
\text { voltammetry }\end{array}$ & $\mathrm{CoS}_{2} /$ Graphene & CA15-3 antigen & LOD: $0.03 \mathrm{U} \mathrm{mL}^{-1}$ & [669] \\
\hline Chronoamperometric & $\mathrm{Pd} / \mathrm{rGO}$ & $\begin{array}{l}\text { prostate-specific } \\
\text { antigen }\end{array}$ & $\begin{array}{c}\text { Sensitivity: } 28.96 \mu \mathrm{A} \mathrm{mL} \\
\mathrm{ng}^{-1} \mathrm{~cm}^{-2}\end{array}$ & {$[670]$} \\
\hline Amperometry & $\mathrm{ZrO}_{2} / \mathrm{rGO}$ & $\begin{array}{l}\text { CYFRA-21-1 } \\
\text { biomarker }\end{array}$ & LOD: $0.122 \mathrm{ng} \mathrm{mL}{ }^{-1}$ & {$[671]$} \\
\hline Amperometry & $\mathrm{Fe}_{3} \mathrm{O}_{4} / \mathrm{GO}$ & $\begin{array}{l}\text { detection of prostate } \\
\text { specific antigen or } \\
\text { prostate specific } \\
\text { membrane antigen }\end{array}$ & $\begin{array}{l}\text { LOD: } 15 \mathrm{fg} / \mathrm{mL} \text { for PSA, } 4.8 \\
\mathrm{fg} / \mathrm{mL} \text { for PSMA }\end{array}$ & {$[672]$} \\
\hline DPASV & $\mathrm{CeO}_{2} /$ Graphene & $\mathrm{Cd}(\mathrm{II})$ & LOD: $0.195 \mathrm{nM}$ & [697] \\
\hline SWASV & $\mathrm{Sn} / \mathrm{rGO}$ & $\mathrm{Cd}(\mathrm{II})$ & LOD: $0.63 \mathrm{nM}$ & {$[698]$} \\
\hline DPASV & $\mathrm{Fe}_{2} \mathrm{O}_{3} / \mathrm{GO}$ & $\mathrm{Cd}(\mathrm{II})$ & LOD: $0.08 \mu \mathrm{g} / \mathrm{L}$ & [699] \\
\hline DPASV & $\mathrm{CeO}_{2} /$ Graphene & $\mathrm{Pb}(\mathrm{II})$ & LOD: $0.10 \mathrm{nM}$ & [697] \\
\hline
\end{tabular}


Table 6. Cont.

\begin{tabular}{|c|c|c|c|c|}
\hline Method & Functionalization & Analyte/Entity & Performances & Reference \\
\hline SWASV & $\mathrm{Sn} / \mathrm{rGO}$ & $\mathrm{Pb}(\mathrm{II})$ & LOD: $0.6 \mathrm{nM}$ & [698] \\
\hline DPASV & $\mathrm{Fe}_{2} \mathrm{O}_{3} / \mathrm{GO}$ & $\mathrm{Pb}(\mathrm{II})$ & LOD: $0.07 \mu g / L$ & [699] \\
\hline DPASV & $\mathrm{CeO}_{2} /$ Graphene & $\mathrm{Hg}(\mathrm{II})$ & LOD: $0.277 \mathrm{nM}$ & [697] \\
\hline DPASV & $\mathrm{CeO}_{2} /$ Graphene & $\mathrm{Cu}(\mathrm{II})$ & LOD: $0.164 \mathrm{nM}$ & [697] \\
\hline SWASV & $\mathrm{Sn} / \mathrm{rGO}$ & $\mathrm{Cu}(\mathrm{II})$ & LOD: $0.52 \mathrm{nM}$ & {$[698]$} \\
\hline $\mathrm{DPV}$ & $\mathrm{Ru} / \mathrm{GO}$ & As(III) & LOD: $2.30 \mathrm{nM}$ & [700] \\
\hline DPASV & Graphene & $\mathrm{Zn}(\mathrm{II})$ & LOD: $1.0 \mu \mathrm{g} / \mathrm{L}$ & [701] \\
\hline DPASV & $\mathrm{Fe}_{2} \mathrm{O}_{3} / \mathrm{GO}$ & $\mathrm{Zn}(\mathrm{II})$ & LOD: $0.11 \mu \mathrm{g} / \mathrm{L}$ & [699] \\
\hline DPV & C nanohorn/GO & 4-nitrochlorobenzene & LOD: $10 \mathrm{nM}$ & {$[681]$} \\
\hline Amperometry & poly(Na styrenesulfonate)/Graphene & Hydrazine & LOD: $1 \mu \mathrm{M} / \mathrm{L}$ & {$[682]$} \\
\hline Amperometry & Reduced Graphene & Sulfides & LOD: $4.2 \mu \mathrm{M}$ & [683] \\
\hline Voltammetry & Polyaniline/Graphene & 4-aminophenol & LOD: $65 \mathrm{nM}$ & {$[684]$} \\
\hline DPV & Polyoxometalate/Graphene & Diphenols & LOD: $40 \mathrm{nM}$ & {$[685]$} \\
\hline Voltammetry & Graphene & Hydroquinone & LOD: $40 \mu \mathrm{M}$ & {$[686]$} \\
\hline Conductometric & Pt/Holey-graphene & $\mathrm{H}_{2}$ & LOD: 20 ppm & [688] \\
\hline Chemoresistive & $\mathrm{Cu} /$ Graphene & $\mathrm{CO}$ & LOD: 0.25 ppm & {$[689]$} \\
\hline Conductometric & Graphene & $\mathrm{CO}_{2}$ & Sensitivity $0.17 \% / \mathrm{ppm}$ & {$[690]$} \\
\hline Optical & Graphene coupled Long-Period Fiber Grating & NO & Sensitivity: 63.65 pm/ppm & {$[691]$} \\
\hline Chemoresistive & PS/Graphene & $\mathrm{NO}_{2}$ & LOD: 0.5 ppm & {$[692]$} \\
\hline Chemoresistive & rGO & $\mathrm{SO}_{2}$ & LOD: 5 ppm & {$[693]$} \\
\hline Chemoresistive & Polyaniline/rGO & $\mathrm{NH}_{3}$ & LOD: 0.2 ppm & {$[694]$} \\
\hline
\end{tabular}

${ }^{1}$ FET: field effect transistor.

Graphene is also utilized for fluorescence detection of substances. Among available techniques, Főrster resonance energy transfer (FRET) may be utilized to localize substances with a resolution of $10 \mathrm{~nm}$. With respect to FRET, carbon nanostructures quench fluorescence over the entire visible region, making them efficient acceptors in FRET. Graphene-sensing is based on fluorescence quenching and several molecules as glucose, $\mathrm{H}_{2} \mathrm{O}_{2}$, dopamine, food toxins and metal ions, can be detected as well as nucleic acids, pathogens and toxins. More information may be found in [702-704].

\section{Conclusions}

Among the elements of the Periodic Table carbon appears as a very special element possessing unique properties and this is the reason why carbon is at the base of the organic chemistry and, in the end, of the life. Among these special properties is the carbon capability to generate rather different structures displaying peculiar properties enabling the development of new biosensors for the detection of a wide variety of biological, organic and inorganic analytes with desirable levels of sensitivity, low detection limits and good stability. The possibility to integrate the carbon nanostructures in other materials as organic polymers or hydrogels or the coupling to metal or oxide nanoparticles constitutes a possible route for increase the detecting possibilities of the sensors or enhance its performances. Moreover, the carbon-based nanostructures may be chemically assembled in 2D and 3Dstructures further providing attributes which improve the sensing capabilities. Finally, carbon nanostructures may be integrated in nano- or micro-scale devices such as microfluidic chips or microelectronic devices which appear particularly promising for the fabrication of devices for the multiple detection of analytes for the miniaturization of the sensors allowing a diffuse sensing to control for example the environment. These advances in biosensor research open interesting perspective for the development of promising plat- 
forms for the detection and control of biomarkers, food quality, toxic substances, pollution, air and environment monitoring.

Funding: This research received no external founding.

Data Availability Statement: All the data presented in this manuscript were derived from the indicated articles which are published in literature and listed in the Reference section.

Acknowledgments: Author would like to thank the Fondazione Bruno Kessler for supporting this work.

Conflicts of Interest: The author declares no conflict of interest.

\section{References}

1. Artunedo, A.; del Toro, R.; Haber, R.E. Consensus-based cooperative control based on pollution sensing and traffic information for urban traffic networks. Sensors 2017, 17, 953. [CrossRef] [PubMed]

2. Kolumban-Antal, G.K.A.; Lasak, V.; Razvan, B.; Groza, B. A secure and portable multi-sensor module for distributed air pollution monitoring. Sensors 2020, 20, 403. [CrossRef] [PubMed]

3. Eifert, T.; Eisen, K.; Maiwald, M.; Herwig, C. Current and future requirements to industrial analytical infrastructure-part 2: Smart sensors. Anal. Bioanal. Chem. 2020, 412, 2037-2045. [CrossRef] [PubMed]

4. Hancke, G.P.; De Carvalho e Silva, B.; Hancke, G.P., Jr. The Role of Advanced Sensing in Smart Cities. Sensors 2013, 13, $393-425$. [CrossRef]

5. Sishodia, R.P.; Ray, R.L.; Singh, S.K. Applications of Remote Sensing in Precision Agriculture: A Review. Remote Sens. 2020, 12, 3136. [CrossRef]

6. Duffy, G.; Regan, F. Recent developments in sensing methods for eutrophying nutrients with a focus on automation for environmental applications. Analyst 2017, 142, 4355-4372. [CrossRef]

7. Lozano, J.; Apetrei, C.; Ghasemi-Varnamkhasti, M.; Matatagui, D.; Santos, J.P. Sensors and Systems for Environmental Monitoring and Control. Hindawi 2017, 2017, 6879748. [CrossRef]

8. Hayat, H.; Griffiths, T.; Brennan, D.; Lewis, R.P.; Barclay, M.; Weirman, C.; Philip, B.; Searle, J.R. The State-of-the-Art of Sensors and Environmental Monitoring Technologies in Buildings. Sensors 2019, 19, 3648. [CrossRef]

9. Gas Sensor Market Size, Share \& Trends Analysis Report By Product (Oxygen/Lambda Sensor, Carbon Dioxide Sensor), by Type (Wired, Wireless), by Technology, by End Use, By Region., and Segment Forecasts, 2021-2028. Available online: https:/ / www.grandviewresearch.com/industry-analysis/gas-sensors-market (accessed on 15 February 2021).

10. Abdel-Karim, R.; Red, Y.; Abdel-Fattah, A. Review-Nanostructured Materials-Based Nanosensors. J. Electrochem. Soc. 2020, 167, 037554. [CrossRef]

11. Kirchner, E.M.; Hirsh, T. Recent developments in carbon-based two-dimensionalmaterials: Synthesis and modification aspectsfor electrochemical sensors. MicroChim. Acta 2020, 187, 441. [CrossRef]

12. Hirsch, A. The era of carbon allotropes. Nat. Mater. 2010, 9, 868-871. [CrossRef]

13. Kreyling, W.G.; Semmler-Behnke, M.; Chaudhry, Q. A complementary definition of nanomaterial. Nano Today 2010, 5, 165-168. [CrossRef]

14. Wang, R.; Chen, D.; Wang, Q.; Ying, Y.; Gao, W.; Xie, L. Recent advances in applications of carbon nanotubes for desalination: A review. Nanomaterials 2020, 10, 1203. [CrossRef] [PubMed]

15. Wu, W.; Shi, Y.; Liu, G.; Fan, X.; Yu, Y. Recent development of graphene oxide based forward osmosis membrane for water treatment: A critical review. Desalination 2020, 491, 114452. [CrossRef]

16. Kanao, E.; Kubo, T.; Otsuka, K. Carbon-Based nanomaterials for Separation Media. Bull. Chem. Soc. Jpn. 2020, 93, 482-489. [CrossRef]

17. Wu, Q.; Lv, H.; Zhao, L. Applications of carbon nanomaterials in chiral separation. Trends Anal. Chem. 2020, $129,115941$. [CrossRef]

18. Pan, X.; Ji, J.; Zhang, N.; Xing, M. Research progress of graphene-based nanomaterials for the environmental remediation. Chin. Chem. Lett. 2020, 31, 1462-1473. [CrossRef]

19. Abd-Elsalam, K.A. Carbon Nanomaterials for Agri-Food and Environmental Applications; Micro and Nanotechnologies; Elsevier Inc.: Amsterdam, The Netherlands, 2020; Volume 1, p. 2.

20. Peng, Z.; Liu, X.; Zhang, W.; Zeng, Z.; Liu, Z.; Zhang, C.; Liu, Y. Advances in the application, toxicity and degradation of carbon nanomaterials in environment: A review. Environ. Int. 2020, 134, 105298. [CrossRef]

21. Peng, L.-M.; Zhang, Z.; Wang, S. Carbon nanotube electronics: Recent advances. Mater. Today 2014, 17, 433-442. [CrossRef]

22. Sengupta, J. Application of Carbon Nanomaterials in the Electronic Industry; Micro and Nano Technologies; Elsevier Science: Amsterdam, The Netherlands, 2020.

23. Yamashita, S. Nonlinear optics in carbon nanotube, graphene, and related 2D materials. APLPhotonics 2014, 4, 034301. [CrossRef]

24. Zhao, Q.; Song, W.; Zhao, B.; Yang, B. Spectroscopic studies of the optical properties of carbon dots: Recent advances and future prospects. Mater. Chem. Front. 2020, 4, 472-488. [CrossRef] 
25. Ghosh, D.; Sarkar, K.; Devi, P.; Kim, K.-H.; Kumar, P. Current and future perspectives of carbon and graphene quantum dots: From synthesis to strategy for building optoelectronic and energy devices. Renew. Sustain. Energy Rev. 2021, 135, 110391. [CrossRef]

26. Jariwala, D.; Sangwan, V.K.; Lauhon, L.J.; Marks, T.J.; Hersam, M.C. Carbon nanomaterials for electronics, optoelectronics, photovoltaics, and sensing. Chem. Soc. Rev. 2013, 42, 2824-2860. [CrossRef] [PubMed]

27. Szeluga, U.; Pusz, S.; Kumanek, B.; Olszowska, K.; Kobyliukh, A.; Trzebicka, B. Effect of graphene filler structure on electrical, thermal, mechanical, and fire retardant properties of epoxy-graphene nanocomposites-A review. Crit. Rev. Sol. Stat. Mater. Sci. 2019, 1-36. [CrossRef]

28. Frank, G.; Lagoudas, D.C. Characterization of electrical and thermal properties of carbon nanotube/epoxy composites. Compos. $B$ 2014, 56, 611-620.

29. Shin, Y.C.; Novin, E.; Kim, H. Electrical and thermal conductivities of carbon fiber composites with high concentrations of carbon nanotubes. Int. J. Precis. Eng. Manufact. 2015, 16, 465-470. [CrossRef]

30. Zhang, X.; Zhao, N.; He, C. The superior mechanical and physical properties of nanocarbon reinforced bulk composites achieved by architecture design-A review. Progr. Mater. Sci. 2020, 113, 100672. [CrossRef]

31. Papageorgiou, D.; Li, Z.; Liu, M.; Kinloch, I.A.; Young, R.J. Mechanisms of reinforcement by graphene and nanotubes in polymer nanocomposites. Nanoscale 2020, 12, 2228-2267. [CrossRef]

32. Hegde, S.; Shenoy, B.S.; Chethan, K.N. Review on carbon fiber reinforced polymer (CFRP) and their mechanical performance. Mater. Today Proc. 2019, 19, 658-662. [CrossRef]

33. Huang, Y.L.G.; Mi, S.W.C.; Wei, S.; Tian, F.; Li, W.; Cao, H.; Cheng, Y. Review on diamond-like carbon films grown by pulsed laser deposition. Appl. Surf. Sci. 2020, 541, 148573.

34. Rekha, M.Y.; Srivastava, C. High corrosion resistance of metal-graphene oxide-metal multilayer coatings. Philos. Mag. 2020, 100, 18-31. [CrossRef]

35. Popescu, C.; Cristea, D.; Bita, B.; Cristescu, R.; Craciun, D.; Chioibasu, G.D.; Luculescu, C.; Paun, I.; Duta, L.; Popescu, A.C. An Experimental Study on Nano-Carbon Films as an Anti-Wear Protection for Drilling Tools. Coatings 2017, 7, 228. [CrossRef]

36. Kausar, A.; Taherian, R. 3-Electrical Conductivity Behavior of Polymer Nanocomposite with Carbon Nanofillers. In Electrical Conductivity in Polymer-Based Composites Experiments, Modelling, and Applications; Plastics Design Library; Elsevier Inc.: Amsterdam, The Netherlands, 2018; pp. 41-72.

37. Lee, J.K.Y.; Chen, N.; Peng, S.; Li, L.; Tian, L.; Thakor, N.; Ramakrishna, S. Polymer-based composites by electrospinning: Preparation \& functionalization with nanocarbons. Progr. Polym. Sci. 2018, 86, 40-84.

38. Zhou, N.; Liu, T.; Wen, B.; Gong, C.; Wei, G.; Su, Z. Recent Advances in the Construction of Flexible Sensors for Biomedical Applications. Biotechnol. J. 2020, 15, 2000094. [CrossRef]

39. Li, C.; Wang, Y.; Jiang, H.; Wang, X. Review-Intracellular Sensors Based on Carbonaceous Nanomaterials: A Review. J. Electrochem. Soc. 2020, 167, 037540. [CrossRef]

40. Kour, R.; Arya, S.; Young, S.-J.; Gupta, V.; Bandhoria, P.; Khosla, A. Review-Recent Advances in Carbon Nanomaterials as Electrochemical Biosensors. J. Electrochem. Soc. 2020, 167, 037555. [CrossRef]

41. Kaur, J.; Gill, G.S.; Jeet, K. Applications of Carbon Nanotubes in Drug Delivery: A Comprehensive Review-Chapter 5. In Characterization and Biology of Nanomaterials for Drug Delivery; Nanoscience and Nanotechnology in Drug Delivery Micro and Nano Technologies; Elsevier Inc.: Amsterdam, The Netherlands, 2019; pp. 113-135.

42. Mendes, R.G.; Bachmatiuk, A.; Büchner, B.; Cuniberti, G.; Rümmeli, M.H. Carbon nanostructures as multi-functional drug delivery platforms. J. Mater. Chem. B 2013, 1, 401-428. [CrossRef] [PubMed]

43. Geim, A.K.; Novoselov, K.S. The rise of graphene. Nat. Mater. 2007, 6, 183-197. [CrossRef]

44. Iijima, S.; Ichihashi, T. Single-shell carbon nanotubes of 1-nm diameter. Nature 1993, 363, 603-605. [CrossRef]

45. Park, S.J.; Lee, S.Y. History and Structure of Carbon Fibers. In Carbon Fibers; Springer series Materials Science; Springer: Dordrecht, The Netherlands, 2015; Volume 210.

46. Kroto, H.W.; Heath, J.R.; O'Brien, S.C.; Curl, R.F.; Smalley, R.E. C60: Buckminsterfullerene. Nature 1985, 318, 162-163. [CrossRef]

47. Suarez-Martinez, I.; Grombert, N.; Ewels, C.P. Nomenclature of sp2 carbon nanoforms. Carbon 2012, 50, 741-747. [CrossRef]

48. Ugarte, D. Curling and closure of graphitic networks under electron-beam irradiation. Nature 1992, 359, 707-709. [CrossRef] [PubMed]

49. Wang, X.; Feng, Y.; Dong, P.; Huang, J. A Mini Review on Carbon Quantum Dots: Preparation, Properties, and Electrocatalytic Application. Front. Chem. 2019, 7, 671. [CrossRef]

50. Danilenko, V.V. On the history of the discovery of nanodiamond synthesis. Phys. Sol. Stat. 2004, 46, 595-599. [CrossRef]

51. Nunn, N.; Torelli, M.; McGuire, G.; Shenderova, O. Nanodiamond: A high impact nanomaterial. Curr. Opin. Sol. Stat. Mater. Sci. 2017, 21, 1-9. [CrossRef]

52. Awschalom, D.D.; Hanson, R.; Wrachtrup, J.; Zhou, B.B. Quantum technologies with optically interfaced solid-state spins. Nat. Photon. 2018, 12, 516-527. [CrossRef]

53. Georgakilas, V.; Perman, J.P.; Tucek, J.; Zboril, R. Broad Family of Carbon Nanoallotropes: Classification, Chemistry, and Applications of Fullerenes, Carbon Dots, Nanotubes, Graphene, Nanodiamonds, and Combined Superstructures. Chem. Rev. 2015, 115, 4744-4822. [CrossRef] [PubMed]

54. Klein, C.A. Pyrolytic Graphites: Their Description as Semimetallic Molecular Solids. J. Appl. Phys. 1962, 33, 3338. [CrossRef] 
55. Mildren, R.P. Intrinsic Optical Properties of Diamond-Chapter 1. In Optical Engineering of Diamond; Wiley-VCH Verlag GmbH \& Co. KGaA: Weinheim, Germany, 2013; pp. 1-34. ISBN 9783527411023.

56. Sergiienko, R.; Shibata, E.; Takashi, H.S.; Akase, N.Z.; Murakami, Y.; Shindo, D. Synthesis of amorphous carbon nanoparticles and carbon encapsulated metal nanoparticles in liquid benzene by an electric plasma discharge in ultrasonic cavitation field. Ultrason. Sonochem. 2006, 13, 6-12. [CrossRef]

57. Posthuma-Trumpie, G.A.; Wichers, J.H.; Koets, M.; Berendsen, L.B.J.M.; van Amerongevn, A. Amorphous carbon nanoparticles: A versatile label for rapid diagnostic (immuno)assays. Anal. Bioanal. Chem. 2012, 402, 593-600. [CrossRef]

58. Xiong, Y.; Xie, Y.; Li, X.; Li, Z. Production of novel amorphous carbon nanostructures from ferrocene in low-temperature solution. Carbon 2004, 42, 1447-1453. [CrossRef]

59. Hu, Z.D.; Hu, Y.F.; Chen, Q.; Duan, X.F.; Peng, L.-M. Synthesis and Characterizations of Amorphous Carbon Nanotubes by Pyrolysis of Ferrocene Confined within AAM Templates. J. Phys. Chem. B 2006, 110, 8263-8267. [CrossRef] [PubMed]

60. Sadeghi, H.; Dorrain, D. Influence of size and morphology on the optical properties of carbon nanostructures. J. Theor. Appl. Phys. 2016, 10, 7-13. [CrossRef]

61. Rakitin, A.; Papadopoulos, C.; Xu, J.M. Electronic properties of amorphous carbon nanotubes. Phys. Rev. B 2000, 61, 5793-5796. [CrossRef]

62. Baimova, J.A.; Liu, B.; Dmitriev, S.V.; Srikanthd, N.; Zhou, K. Mechanical properties of bulk carbon nanostructures: Effect of loading and temperature. Phys. Chem. Chem. Phys. 2014, 16, 19505-19513. [CrossRef] [PubMed]

63. Cataldo, F.; Da Ros, T. Medicinal Chemistry and Pharmacological Potential of Fullerenes and Carbon Nanotubes; Carbon Materials in Chemistry and Physics; Springer Business Science; Springer: Berlin/Heidelberg, Germany, 2008.

64. Semenov, K.N.; Charykov, N.A.; Keskinov, V.A.; Piartman, A.K.; Blokhin, A.A.; Kopyrin, A.A. Solubility of light fullerenes in organic solvents. J. Chem. Eng. Data 2010, 55, 13-36. [CrossRef]

65. Rasovic, I. Water-soluble fullerenes for medical applications. Mater. Sci. Technol. 2017, 33, 777-794. [CrossRef]

66. Lieber, C.M.; Chen, C.-C. Preparation of Fullerenes and Fullerene-Based Materials. Sol. Stat. Phys. 1994, 48, 109-148.

67. Hare, J.P.; Kroto, H.W.; Taylor, R. Preparation and UV/visible spectra of fullerenes C60 and C70. Chem. Phys. Lett. 1991, 177, 394-398. [CrossRef]

68. Parker, D.H.; Wurz, P.; Chatterjee, K.; Lykke, K.R.; Hunt, J.E.; Pellin, M.J.; Hemminger, J.C.; Gruen, D.M.; Stock, L.M. High-yield synthesis, separation, and mass-spectrometric characterization of fullerenes C60 to C266. J. Am. Chem. Soc. 1991, 113, 7499-7503. [CrossRef]

69. Kroto, H.W. Space, Stars, C60, and Soot. Science 1988, 242, 1139-1145. [CrossRef] [PubMed]

70. Taylor, R.; Langley, G.J.; Kroto, H.W.; Walton, D.R.M. Formation of C60 by pyrolysis of naphthalene. Nature 1993, 366, 728-731. [CrossRef]

71. Howard, J.B.; McKinnon, J.T.; Makarovsky, Y.; Lafleur, A.L.; Johnson, M.E. Fullerenes C60 and C70 in flames. Nature 1991, 352, 139-141. [CrossRef]

72. Homann, K.H. Fullerenes and Soot Formation- New Pathways to Large Particles in Flames. Angew. Chem. Int. Ed. 1998, 27, 2434-2451. [CrossRef]

73. Murayama, H.; Tomonoh, S.; Alford, J.M.; Karpuk, M.E. Fullerene production in tons and more: From science to industry. Fuller Nanotub. Carbon Nanostruct. 2005, 12, 1-9. [CrossRef]

74. Stalling, D.L.; Kuo, K.C.; Guo, C.Y.; Saim, S. Separation of Fullerenes C60, C70, and C76-84 on Polystyrene Divinylbenzene Columns. J. Liq. Chromat. 1993, 16, 699-722. [CrossRef]

75. Gonzalez-Martinez, I.G.; Bachmatiuk, A.; Bezugly, V.; Kunstmann, J.; Gemming, T.; Liu, Z.; Cunibertic, G.; Rummeli, M.H. Electron-beam induced synthesis of nanostructures: A review. Nanoscale 2016, 8, 11340-11362. [CrossRef]

76. Troiani, H.E.; Camacho-Bragado, A.; Armendariz, V.; Gardea Torresday, J.L.; Yacaman, M.J. Synthesis of Carbon Onions by Gold Nanoparticles and Electron Irradiation. Chem. Mater. 2003, 15, 1029-1031. [CrossRef]

77. De Herr, W.A.; Ugarte, D. Carbon onions produced by heat treatment of carbon soot and their relation to the $217.5 \mathrm{~nm}$ interstellar absorption feature. Chem. Phys. Lett. 1993, 207, 480-486. [CrossRef]

78. Sano, N.; Wang, H.; Chhowalla, M.; Alexandrou, I.; Amaratunga, G.A. Synthesis of carbon “onions" in water. Nature 2001, 414, 506-507. [CrossRef]

79. Alexandrou, I.; Wang, H.; Sano, N.; Amaratunga, G.A. Structure of carbon onions and nanotubes formed by arc in liquids. J. Chem. Phys. 2004, 120, 1055-1058. [CrossRef]

80. Plonska-Brzezinska, M.E.; Lapinski, A.; Wilczewska, A.Z.; Dubis, A.T.; Villalta-Cerdas, A.; Winkler, K.; Echegoyen, L. The synthesis and characterization of carbon nano-onions produced by solution ozonolysis. Carbon 2011, 49, 5079-5089. [CrossRef]

81. Bian, Y.; Liu, L.; Liu, D.; Zhu, Z.; Shao, Y.; Li, M. Electrochemical synthesis of carbon nano onions. Inorg. Chem. Front. 2020, 7, 4404-4411. [CrossRef]

82. Haddon, R.C.; Palmer, R.E.; Kroto, H.W.; Sermon, P.A. The fullerenes: Powerful carbon-based electron acceptors. Philos. Trans. R Soc. A 1993, 343, 53-62.

83. Hirsch, A. Functionalization of fullerenes and carbon nanotubes. Phys. Stat. Sol. 2006, 243, 3209-3212. [CrossRef]

84. Weibo, Y.; Seifermann, S.M.; Pierrat, P.; Brase, S. Synthesis of highly functionalized C60 fullerene derivatives and their applications in material and life sciences. Org. Biomol. Chem. 2015, 13, 25-54.

85. Samal, S.; Sahoo, S.K. An overview of fullerene chemistry. Bull. Mater. Sci. 1997, 20, 141-230. [CrossRef] 
86. Jensen, A.W.; Wilson, S.R.; Schuster, D.I. Biological applications of fullerenes. Bioorg. Med. Chem. 1996, 4, 767-779. [CrossRef]

87. Kadish, K.M.; Ruoff, R.S. (Eds.) Fullerenes: Chemistry, Physics, and Technology, 1st ed.; Wiley-Interscience: New York, NY, USA, 2000; ISBN-13: 978-0471290896.

88. Diederich, F.; Gomez-Lopez, M. Supramolecular fullerene chemistry. Chem. Soc. Rev. 1999, 28, 263-277. [CrossRef]

89. Ikeda, A.; Iizuka, T.; Maekubo, N.; Aono, R.; Kikuchi, J.; Akiyama, M.; Konishi, T.; Ogawa, T.; Ishida-Kitagawa, N.; Tatebe, H.; et al. Cyclodextrin Complexed [60]Fullerene Derivatives with High Levels of Photodynamic Activity by Long Wavelength Excitation. ACS Med. Chem. Lett. 2013, 4, 752-756. [CrossRef]

90. Chiang, L.Y.; Swirczewski, J.W.; Hsu, C.S.; Chowdhury, S.K.; Cameron, S.; Creegan, K. Multi-hydroxy additions onto C60 fullerene molecules. J. Chem. Soc. Chem. Commun. 1992, 1791-1793. [CrossRef]

91. Li, J.; Takeuchi, A.; Ozawa, M.; Li, X.; Saigo, K.; Kitazawa, K. C60 fullerol formation catalysed by quaternary ammonium hydroxides. J. Chem. Soc. Chem. Commun. 1993, 1784-1785. [CrossRef]

92. Arrais, A.; Diana, E. Highly water soluble C60 derivatives: A new synthesis. Fuller. Nanotub. Carbon Nanostruct. 2003, 11, 35-46. [CrossRef]

93. Kokubo, K.; Matsubayashi, K.; Tategaki, H.; Takada, H.; Oshima, T. Facile Synthesis of Highly Water-Soluble Fullerenes More Than Half-Covered by Hydroxyl Groups. ACS Nano 2008, 2, 327-333. [CrossRef] [PubMed]

94. Chiang, L.Y.; Wang, L.-Y.; Swirczewski, J.W.; Soled, S.; Cameron, S. Efficient Synthesis of Polyhydroxylated Fullerene Derivatives via Hydrolysis of Polycyclosulfated Precursors. J. Org. Chem. 1994, 59, 3960-3968. [CrossRef]

95. Chiang, L.Y.; Bhonsle, J.B.; Wang, L.; Shu, S.F.; Chang, T.M.; Hwu, J.R. Efficent One-Flask Synthesis of Water-Soluble [60]Fullerenols. Tetraedron 1996, 52, 4963-4972. [CrossRef]

96. Schneider, N.S.; Darwish, A.D.; Kroto, H.W.; Taylor, R.; Walton, D.R.M. Formation of Fullerenols via Hydroboration of FullereneC60. J. Chem. Soc. Chem. Commun. 1994, 463-464. [CrossRef]

97. Wang, S.; He, P.; Zhang, J.-M.; Jiang, H.; Zhu, S.-Z. Novel and Efficient Synthesis of Water-Soluble [60]Fullerenol by Solvent-Free Reaction. Synth. Commun. 2005, 35, 1803-1807. [CrossRef]

98. Xing, G.; Zhang, J.; Zhao, Y.; Tang, J.; Zhang, B.; Gao, X.; Yuan, H.; Qu, L.; Cao, W.; Chai, Z. Influences of Structural Properties on Stability of Fullerenols. J. Phys. Chem. B 2004, 108, 11473-11479. [CrossRef]

99. Wudl, F.; Hirsh, A.; Khemani, K.C.; Suzuki, T.; Allemand, P.M.; Koch, A.; Eckert, H.; Srdanov, G.; Webb, A.M. Survey of chemical reactivity of C60, electrophile, and dienopolarophile par excellence. In Chapter 11-Synthesis, Properties, and Chemsitry of Large Carbon Clusters; American Chemical Society: Washington, DC, USA, 1992; pp. 161-176.

100. Seshadri, R.; Govindaraj, A.; Nagarajan, R.; Pradeep, T.; Rao, C.N.R. Addition of amines and halogens to fullerenes C60 and C70. Tetrahedron 1992, 33, 2069-2070. [CrossRef]

101. Maggini, M.; Scorrano, G.; Prato, M. Addition of azomethine ylides to C60: Synthesis, characterization, and functionalization of fullerene pyrrolidines. J. Am. Chem Soc. 1993, 115, 9798-9799. [CrossRef]

102. Kampe, K.-D.; Egger, N.; Vogel, M. Diamino and Tetraamino Derivatives of Buckminsterfullerene C60. Angew. Chem. Int. Ed. 1993, 32, 1174-1176. [CrossRef]

103. Hirsh, A.; Li, Q.; Wudl, F. Globe-trotting Hydrogens on the Surface of the Fullerene Compound C60H6(N(CH2CH2)2O)6. Angew. Chem. Int. Ed. 1991, 30, 1309-1310. [CrossRef]

104. Hwu, J.R.; Kuo, T.Y.; Chang, T.M.; Patel, H.V.; Yong, K.T. Amination of Buckminsterfullerene C60 at Low Temperature: Application in Polyamide Synthesis. Fuller. Sci. Technol. 1996, 4, 407-422. [CrossRef]

105. Li, Y.; Gan, L. Selective Addition of Secondary Amines to C60: Formation of Penta- and Hexaamino[60]fullerenes. J. Org. Chem. 2014, 79, 8912-8916. [CrossRef]

106. Okuyama, M.; Teramoto, A.; Watanabe, M.; Inomata, H. Solvent-free chemical modification of fullerene C60 by an aminoterminated polyethylene glycol. Tanso 2008, 235, 268-274. [CrossRef]

107. Peng, H.; Lam, J.W.Y.; Leung, F.S.; Poon, T.W.H.; Wu, A.X.; Yu, N.-T.; Tang, B.Z. Synthesis of fullerene-containing sol-gel glasses. J. Sol. Gel. Sci. 2001, 22, 205-218. [CrossRef]

108. Miller, G.P. Reactions between aliphatic amines and [60]fullerene: A review. Comptes Rendus Chim. 2006, 9, 952-959. [CrossRef]

109. Bingel, C. Cyclopropanierung von fullerenen. Chem. Ber. 1993, 126, 1957-1959. [CrossRef]

110. Li, H.; Haque, S.A.; Kitaygorodskiy, A.; Meziani, M.J.; Torres-Castillo, M. Alternatively Modified Bingel Reaction for Efficient Syntheses of C60 Hexakis-Adducts. Org. Lett. 2006, 8, 5641-5643. [CrossRef]

111. Torosyan, S.A.; Biglova, Y.N.; Mikheev, V.V.; Khalitova, Z.T.; Gimalova, F.A.; Miftakhov, M.S. Synthesis of fullerene-containing methacrylates. Mendeleev Commun. 2012, 22, 199-200. [CrossRef]

112. Pereira, G.R.; Santos, L.J.; Luduvico, I.; Alves, R.B.; Pereira de Freitas, R. Click' chemistry as a tool for the facile synthesis of fullerene glycoconjugate derivatives. Tetrahedron Lett. 2010, 51, 1022-1025. [CrossRef]

113. Riala, M.; Chronakis, N. A Facile Access to Enantiomerically Pure [60]Fullerene Bisadducts with the Inherently Chiral Trans-3 Addition Pattern. Org. Lett. 2011, 13, 2844-2847. [CrossRef] [PubMed]

114. Jin, B.; Shen, J.; Peng, R.; Zheng, R.; Chu, S. Efficient cyclopropanation of [60]fullerene starting from bromo-substituted active methylene compounds without using a basic catalyst. Tetrahedron Lett. 2014, 55, 5007-5010. [CrossRef]

115. Thilgen, C.; Diederich, F. Tether-directed remote functionalization of fullerenes C60 and C70. Comptes Rendus Chim. 2006, 9 , 868-880. [CrossRef] 
116. Wudl, F. The chemical properties of buckminsterfullerene (C60) and the birth and infancy of fulleroids. Acc. Chem. Res. 1992, 25, 157-161. [CrossRef]

117. Martin, N.; Segura, J.L.; Wudl, F. New Concepts in Diels-Alder Cycloadditions to Fullerenes. In Fullerenes: From Synthesis to Optoelectronic Properties. Developments in Fullerene Science; Springer: Dordrecht, The Netherlands, 2002; Volume 4.

118. Diederich, F.; Thiilgen, C. Covalent Fullerene Chemistry. Science 1996, 271, 317-324. [CrossRef]

119. Goodarzi, S.; Da Ros, T.; Conde, J.; Sefat, F.; Mozafari, M. Fullerene: Biomedical engineers get to revisit an old friend. Mater. Today 2017, 20, 460-480. [CrossRef]

120. Margetic, D.; Strukil, V. Chapter 7-Applications of Ball Milling in Nanocarbon Material Synthesis. In Mechanochemical Organic Synthesis; Elsevier: Amsterdam, The Netherlands, 2016; pp. 323-342. ISBN 9780128021842.

121. Maquin, B.; Derre, A.; Labrugere, C.; Trinquecoste, M.; Chadeyron, P.; Delhaes, P. Submicronic powders containing carbon, boron and nitrogen: Their preparation by chemical vapour deposition and their characterization. Carbon 2000, 38, 145-156. [CrossRef]

122. Tsai, S.; Lee, C.; Chao, C.; Shih, H. A novel technique for the formation of carbon-encapsulated metal nanoparticles on silicon. Carbon 2000, 38, 781-785. [CrossRef]

123. Hou, S.-S.; Chung, D.-H.; Lin, T.-H. High-yield synthesis of carbon nano-onions in counter flow diffusion flames. Carbon 2009, 47, 938-947. [CrossRef]

124. Choucair, M.; Stride, J.A. The gram-scale synthesis of carbon onions. Carbon 2012, 50, 1109-1115. [CrossRef]

125. Zhao, M.; Song, H.; Chen, X.; Lian, W. Large-scale synthesis of onion-like carbon nanoparticles by carbonization of phenolic resin. Acta Mater. 2007, 55, 6144-6150. [CrossRef]

126. Gao, Y.; Zhou, Y.; Park, J.; Wang, H.; He, X.N.; Luo, H.; Jiang, L.; Lu, Y.F. Resonant excitation of precursor molecules in improving the particle crystallinity, growth rate and optical limiting performance of carbon nano-onions. Nanotechnology 2011, $22,165604$. [CrossRef] [PubMed]

127. Chung, D.-H.; Lin, T.-H.; Hou, S.-S. Flame synthesis of carbon nano-onions enhanced by acoustic modulation. Nanotechnology 2010, 21, 435604. [CrossRef] [PubMed]

128. Tomita, S.; Burian, A.; Dore, J.C.; LeBolloch, D.; Fujii, M.; Hayashi, S. Diamond nanoparticles to carbon onions transformation: X-ray diffraction studies. Carbon 2002, 40, 1469-1474. [CrossRef]

129. Zou, Q.; Li, Y.G.; Lv, B.; Wang, M.Z.; Zou, L.H.; Zhao, Y.C. Transformation of onion-like carbon from nanodiamond by annealing. Inorg. Mater. 2010, 46, 127-131. [CrossRef]

130. Tomita, S.; Sakurai, T.; Ohta, H.; Fujii, M.; Hayashi, S. Structure and electronic properties of carbon onions. J. Chem. Phys. 2001, 114, 7477-7482. [CrossRef]

131. Zuaznabar-Gardona, J.C.; Fragoso, A. Determination of the Hansen solubility parameters of carbon nano-onions and prediction of their dispersibility in organic solvents. J. Mol. Liq. 2019, 294, 111646. [CrossRef]

132. Olena, M.; Zubyk, H.; Plonska-Brzezinska, M.E. Carbon nano-onions: Unique carbon nanostructures with fascinating properties and their potential applications. Inorg. Chim. Acta 2017, 468, 49-66.

133. Rettenbacher, A.S.; Elliot, B.; Hudson, J.S.; Amirkhanian, A.; Echegoyen, L. Preparation and Functionalization of Multilayer Fullerenes (Carbon Nano-Onions). Chem. Eur. J. 2006, 12, 376-387. [CrossRef]

134. Basiuk, E.V.; Monroy-Pelaez, M.; Puente-Lee, I.; Basiuk, V.A. Direct Solvent-Free Amination of Closed-Cap Carbon Nanotubes: A Link to Fullerene Chemistry. Nano Lett. 2004, 4, 863-866. [CrossRef]

135. Georgakilas, V.; Guldi, D.M.; Signorini, R.; Bozio, R.; Prato, M. Organic Functionalization and Optical Properties of Carbon Onions. J. Am. Chem. Soc. 2003, 125, 14268-14269. [CrossRef]

136. Palkar, A.; Melin, F.; Cardona, C.M.; Elliot, B.; Naskar, A.K.; Edie, D.D.; Kumbhar, A.; Echegoyen, L. Reactivity differences between carbon nano onions (CNOs) prepared by different methods. Chem. Asian J. 2007, 2, 625-633. [CrossRef] [PubMed]

137. Molina-Ontoria, A.; Chaur, M.N.; Plonska-Brzezinska, M.E.; Echegoyen, L. Preparation and characterization of soluble carbon nano-onions by covalent functionalization, employing a Na-K alloy. Chem. Commun. 2013, 49, 2406-2408. [CrossRef] [PubMed]

138. Palkar, A.; Kumbhar, A.; Athans, A.J.; Echegoyen, L. Pyridyl-Functionalized and Water-Soluble Carbon Nano Onions: First Supramolecular Complexes of Carbon Nano Onions. Chem. Mater. 2008, 20, 1685-1687. [CrossRef]

139. Sek, S.; Breczko, J.; Plonska-Brzezinska, M.E.; Wilczewska, A.Z.; Echegoyen, L. STM-Based Molecular Junction of Carbon Nano-Onion. Chem. Phys. Chem. 2013, 14, 96-100. [CrossRef]

140. Kordatos, K.; Da Ros, T.; Bosi, S.; Vázquez, E.; Bergmin, M.; Cusan, C.; Pellarini, F.; Tomberli, V.; Baiti, B.; Pantarotto, D.; et al. Novel Versatile Fullerene Synthons. J. Org. Chem. 2001, 66, 4915-4920. [CrossRef] [PubMed]

141. Frasconi, M.; Marotta, R.; Markey, L.; Flavin, K.; Spampinato, V.; Ceccone, G.; Echegoyen, L. Multi-Functionalized Carbon Nano-onions as Imaging Probes for Cancer Cells. Chem. Eur. J. 2015, 21, 19071-19080. [CrossRef]

142. Mykhailiv, O.; Imierska, M.; Petelczyc, M.; Echegoyen, L. Chemical versus electrochemical synthesis of carbon nanoonion/polypyrrole composites for supercapacitor electrodes. Chem. A Eur. J. 2015, 21, 5783-5793. [CrossRef]

143. Plonska-Brzezinska, M.E.; Brus, D.M.; Breczko, J.; Echegoyen, L. Carbon Nano-Onions and Biocompatible Polymers for Flavonoid Incorporation. Chem. Eur. J. 2013, 19, 5019-5024. [CrossRef]

144. Bartelmess, J.; Giordani, S. Carbon nano-onions (multi-layer fullerenes): Chemistry and applications. Beilstein J. Nanotechnol. 2014, 5, 1980-1998. [CrossRef]

145. Berdinsky, A.S.; Shevtsov, Y.V.; Okotrub, A.V.; Trubin, S.V.; Chadderton, L.T.; Fink, D.; Lee, J.H. Sensor Properties of Fullerene Films and Fullerene Compounds with Iodine. Chem. Sustain. Dev. 2000, 8, 141-146. 
146. Saha, D.; Das, S. Development of Fullerene Modified Metal Oxide Thick Films for Moisture Sensing Application. Mater. Today Proc. 2018, 5, 9817-9825. [CrossRef]

147. Hammond, J.L.; Formisano, N.; Estrela, P.; Carrara, S.; Tkac, J. Electrochemical biosensors and nanobiosensors. Essay Biochem. 2016, 60, 69-80.

148. Pereira, A.C.; Sales, M.G.F.; Rodrigues, L.R. Biosensors for Rapid Detection of Breast Cancer Biomarkers-Chapter 3. In Advanced Biosensors for Health Care Applications; Elsevier: Amsterdam, The Netherlands, 2019; pp. 71-103.

149. Sherigara, B.S.; Kutner, W.; Da Souza, F. Electrocatalytic Properties and Sensor Applications of Fullerenes and Carbon Nanotubes. Elecroanalysis 2003, 15, 753-772. [CrossRef]

150. Huang, Y.; Wayner, D.M. Selective catalytic debromination by C60 mono-, di-, and trianion. J. Am. Chem Soc. 1993, 115, 367-368. [CrossRef]

151. Diamond, D. (Ed.) Principles of Chemical and Biological Sensors; Wiley: New York, NY, USA, 1993.

152. Rogers, K.R.; Mulchandani, A.; Zhou, W. (Eds.) Biosensors and Chemical Sensor Technology; American Chemical Society: Los Angeles, CA, USA, 1995.

153. Furuuchi, N.; Shrestha, R.G.; Yamashita, Y.; Hirao, T.; Ariga, K.; Shrestha, L.K. Self-Assembled Fullerene Crystals as Excellent Aromatic Vapor Sensors. Sensors 2019, 19, 267. [CrossRef]

154. Rather, J.R.; De Wael, K. Fullerene-C60 sensor for ultra-high sensitive detection of bisphenol-A and its treatment by green technology. Sens. Actuators B 2013, 176, 110-117. [CrossRef]

155. Shih, J.S. Piezoelectric Crystal Membrane Chemical Sensors Based on Fullerene and Macrocyclic Polyethers. J. Chin. Chem. Soc. 2000, 47, 21-32. [CrossRef]

156. Shi, Z.-L.; Mao, Y.-P.; Tong, W.; Jin, L.T. Electrochemical behavior of C60 modified electrode in aqueous solutions. Chin. J. Chem. 1994, 12, 117-122. [CrossRef]

157. Augusto, V.; Baleizao, C.; Berberan-Santos, M.N.; Farinha, J.P. Oxygen-proof fluorescence temperature sensing with pristine C70 encapsulated in polymer nanoparticles. J. Mater. Chem. 2010, 20, 1192-1197. [CrossRef]

158. Baleizao, C.; Nagl, S.; Schaferling, M.; Berberan-Santos, M.N.; Wolbeis, O.S. Dual fluorescence sensor for trace oxygen and temperature with unmatched range and sensitivity. Anal. Chem. 2008, 80, 6449-6457. [CrossRef]

159. Kochmann, S.; Beleizao, C.; Berberan-Santos, M.N.; Wolbeis, O.S. Sensing and Imaging of Oxygen with Parts per Billion Limits of Detection and Based on the Quenching of the Delayed Fluorescence of $13 \mathrm{C} 70$ Fullerene in Polymer Hosts. Anal. Chem. 2013, 85, 1300-1304. [CrossRef] [PubMed]

160. Rather, J.A.; Al Harthi, A.J.; Khudaish, E.A.; Qurashi, A.; Munama, A.; Kannanc, P. An electrochemical sensor based on fullerene nanorods for the detection of paraben, an endocrine disruptor. Anal. Methods 2016, 8, 5690-5700. [CrossRef]

161. Wu, H.; Fan, S.; Jin, X.; Zhang, H.; Chen, H.; Dai, Z.; Zou, X. Construction of a Zinc Porphyrin-Fullerene-Derivative Based Nonenzymatic Electrochemical Sensor for Sensitive Sensing of Hydrogen Peroxide and Nitrite. Anal. Chem. 2014, 86, 6285-6290. [CrossRef]

162. Yang, X.; Ebrahimi, A.; Li, A.; Cui, Q. Fullerene-biomolecule conjugate and their biomedical applications. Int. J. Nanomed. 2014, 9 , 77-92. [CrossRef]

163. Pang, D.-W.; Zhao, Y.-D.; Fang, P.-F.; Cheng, J.-K.; Chen, Y.-Y.; Qi, Y.-P.; Abruna, H.D. Interactions between DNA and a watersoluble C60 derivative studied by surface-based electrochemical methods. J. Electroanal. Chem. 2004, 567, 339-349. [CrossRef]

164. Zhou, L.; Wang, T.; Bai, Y.; Li, Y.; Qiu, J.; Yu, W.; Sheng, Z. Dual-amplified strategy for ultrasensitive electrochemical biosensor based on click chemistry-mediated enzyme-assisted target recycling and functionalized fullerene nanoparticles in the detection of microRNA-141. Biosens. Bioelectron. 2020, 150, 111964. [CrossRef]

165. Shiraishi, H.; Itoh, T.; Takagi, K.; Sakane, M.; Mori, T.; Wang, J. Electrochemical detection of E. coli 16s rDNA sequence using air-plasma-activated fullerene-impregnated screen printed electrodes. Bioelectrochem. 2007, 70, 481-487. [CrossRef]

166. Uygun, Z.O.; Pahin, C.; Yilmaz, M.; Akçay, Y.; Akdemir, A.; Sağın, F. Fullerene-PAMAM(G5) composite modified impedimetric biosensor to detect Fetuin-A in real blood samples. Anal. Biochem. 2018, 542, 11-15. [CrossRef]

167. Bai, L.; Chen, Y.; Bai, Y.; Chen, Y.; Zhou, J.; Huang, A. Fullerene-doped polyaniline as new redox nanoprobe and catalyst in electrochemical aptasensor for ultrasensitive detection of Mycobacterium tuberculosis MPT64 antigen in human serum. Biomaterials 2017, 133, 11-19. [CrossRef] [PubMed]

168. Gao, Y.-F.; Yang, T.; Yang, X.-L.; Zhang, Y.-S.; Xiao, B.-L.; Hong, J.; Sheibani, N.; Ghourchian, H.; Hong, T.; Moosavi-Movahedi, A.A. Direct electrochemistry of glucose oxidase and glucose biosensing on a hydroxyl fullerenes modified glassy carbon electrode. Biosens. Bioelectron. 2014, 60, 30-34. [CrossRef]

169. Lin, L.-H.; Shih, J.S. Immobilized fullerene C60-enzyme-based electrochemical glucose sensor. J. Chin. Chem. Soc. 2011, 58, 228-235. [CrossRef]

170. Chuang, C.-W.; Shih, J.S. Preparation and application of immobilized C60-glucose oxidase enzyme in fullerene C60-coated piezoelectric quartz crystal glucose sensor. Sens. Actuatoas B 2001, 81, 1-8. [CrossRef]

171. Kasra, S.; Lee, Y.H.; Tan, L.L.; Majid, R. Potentiometric Urea Biosensor Based on an Immobilised Fullerene-Urease Bio-Conjugate. Sensors 2013, 13, 16851-16866.

172. Zhong, X.; Yuan, R.; Chai, Y. In situ spontaneous reduction synthesis of spherical Pd@Cys-C 60 nanoparticles and its application in nonenzymatic glucose biosensors. Chem. Commun. 2012, 48, 597-599. [CrossRef] 
173. Anusha, T.; Bhavani, K.S.; Kumar, J.V.S.; Brahman, P.K. Designing and fabrication of electrochemical nanosensor employing fullerene-C60 and bimetallic nanoparticles composite film for the detection of vitamin D3 in blood samples. Diam. Rel. Mater. 2020, 104, 107761. [CrossRef]

174. Shetti, N.P.; Malode, S.J.; Nandibewoor, S.T. Electrochemical behavior of an antiviral drug acyclovir at fullerene-C60-modified glassy carbon electrode. Bioelectrochemistry 2012, 88, 76-83. [CrossRef] [PubMed]

175. Ertugrul, Y.; Uygun, H.D.; Uygun, Z.O.; Canbay, E.; Sagin, F.G.; Sezer, E. Non-invasive cortisol detection in saliva by using molecularly cortisol imprinted fullerene-acrylamide modified screen printed electrodes. Talanta 2020, 206, 120225. [CrossRef]

176. Sharma, P.S.; Dabrowski, M.; Noworyta, K.; Huynh, T.-P.; Kc, C.; Sobczak, J.W.; D’Souza, F.; Kutner, W. Fullerene derived molecularly imprinted polymer for chemosensing of adenosine-5â $€^{2}$ - triphosphate (ATP). Anal. Chim. Acta 2014, 844, 61-69. [CrossRef]

177. Pan, N.-Y.; Shih, J.-S. Piezoelectric crystal immunosensors based on immobilized fullerene C60-antibodies. Sens. Actuators B 2004, 98, 180-187. [CrossRef]

178. Hashmi, M.A.; Lein, M. Carbon Nano-onions as Photosensitizers: Stacking-Induced Red-Shift. J. Phys. Chem. C 2018, 122, 2422-2431. [CrossRef]

179. Maffeis, V.; Moni, L.; Di Stefano, D.; Giordani, S.; Riva, R. Diversity-oriented synthesis of blue emissive nitrogen heterocycles and their conjugation with carbon nano-onions. Front. Chem. Sci. Eng. 2020, 14, 76-89. [CrossRef]

180. Olariu, M.; Arcire, A. Electrostimulated Desorption Hydrogen Sensor Based on Onion-Like Carbons as a Sensing Element. J. Electron. Mater. 2018, 47, 6476-6483. [CrossRef]

181. Buryakov, T.I.; Romanenko, A.I.; Anikeeva, O.B.; Tkachev, E.N.; Kuznetsov, V.L.; Usoltseva, A.N.; Moseenkov, S.I.; Mazov, I.N.; Ischenko, A.V. Temperature dependencies of conductivity of multi-walled carbon nanotubes and onion-like carbon in different gaseous medium. Int. J. Nanosci. 2009, 8, 19-22. [CrossRef]

182. Bo, X.; Bai, J.; Ju, J.; Guo, L. A sensitive amperometric sensor for hydrazine and hydrogen peroxide based on palladium nanoparticles/onion-like mesoporous carbon vesicle. Anal. Chim. Acta 2010, 675, 29-35. [CrossRef]

183. Raghu, A.V.; Karuppanan, K.K.; Pullithadathil, B. Highly Surface Active Phosphorus-Doped Onion-Like Carbon Nanostructures: Ultrasensitive, Fully Reversible, and Portable NH3 Gas Sensors. ACS Appl. Electron. Mater. 2019, 1, 2208-2219. [CrossRef]

184. Zuaznabar-Gardona, J.C.; Fragoso, A. A wide-range solid state potentiometric $\mathrm{pH}$ sensor based on poly-dopamine coated carbon nano-onion electrodes. Sens. Actuators B 2018, 273, 664-671. [CrossRef]

185. Tripathi, K.M.; Bhati, A.; Singh, A.; Gupta, N.R.; Verma, S.; Sarkar, S.; Sonkar, S.K. From the traditional way of pyrolysis to tunable photoluminescent water soluble carbon nanoonions for cell imaging and selective sensing of glucose. RSC Adv. 2016, 6 , 37319-37329. [CrossRef]

186. Sonkar, S.K.; Ghosh, M.; Roy, M.; Begum, A.; Sarkar, S. Carbon Nano-Onions as Nontoxic and High-Fluorescence Bioimaging Agent in Food Chain-An In Vivo Study from Unicellular E. coli to Multicellular C. elegans. Mater. Express 2012, 2, 105-114. [CrossRef]

187. Li, X.; Liu, Y.; Song, X.; Wang, H.; Gu, H.; Zeng, H. Intercrossed Carbon Nanorings with Pure Surface States as Low-Cost and Environment-Friendly Phosphors for White-Light-Emitting Diodes. Angew. Chem. Int. Ed. 2015, 54, 1759-1764. [CrossRef]

188. Vini, S. Natural source derived carbon nano-onions as electrode material for sensing applications. Diam. Rel. Mater. 2018, 87, 202-207.

189. Mohapatra, J.; Ananthoju, B.; Nair, V.; Mitra, A.; Bahadur, D.; Medhekar, N.V.; Aslam, M. Enzymatic and non-enzymatic electrochemical glucose sensor based on carbon nano-onions. Appl. Surf. Sci. 2018, 442, 332-341. [CrossRef]

190. Bartolome, J.P.; Echegoyen, L.; Fragoso, A. Reactive Carbon Nano-Onion Modified Glassy Carbon Surfaces as DNA Sensors for Human Papillomavirus Oncogene Detection with Enhanced Sensitivity. Anal. Chem. 2015, 87, 6744-6751. [CrossRef]

191. Yang, J.; Zhang, Y.; Kim, D.Y. Electrochemical sensing performance of nanodiamond-derived carbon nano-onions: Comparison with multiwalled carbon nanotubes, graphite nanoflakes, and glassy carbon. Carbon 2016, 98, 74-82. [CrossRef]

192. Ozoemena, O.C.; Shai, L.J.; Maphumulo, T.; Ozoemena, K.I. Electrochemical Sensing of Dopamine Using Onion-like Carbons and Their Carbon Nanofiber Composites. Electrocatalysis 2019, 10, 381-391. [CrossRef]

193. Luszczyn, J.; Plonska-Brzezinska, M.E.; Palkar, A.; Dubis, A.T.; Simionescu, A.; Simionescu, D.T.; Kalska-Szostko, B.; Winkler, K.; Echegoyen, L. Small Noncytotoxic Carbon Nano-Onions: First Covalent Functionalization with Biomolecules. Chem. Eur. J. 2010, 16, 4870-4880. [CrossRef]

194. Shahin, D.I.; Anderson, T.J.; Feygelson, T.I.; Pate, B.B.; Wheeler, V.D.; Greenlee, J.D.; Hite, J.K.; Tadjer, M.J.; Christou, A.; Hobart, K.D. Thermal etching of nanocrystalline diamond films. Diam. Rel. Mater. 2015, 59, 116-121. [CrossRef]

195. Graebner, J.E. Thermal Conductivity of Diamond. In Diamond: Electronic Properties and Applications; The Kluwer International Series in Engineering and Computer Science (Electronic Materials: Science and Technology); Springer: Boston, MA, USA, 1995.

196. Painter, G.S.; Ellis, D.E.; Lubinsky, A.R. Ab-Initio Calculation of the Electronic Structure and Optical Properties of Diamond Using the Discrete Variational Method. Phys. Rev. B 1971, 4, 3610-3622. [CrossRef]

197. Lu, H.C.; Lin, M.Y.; Chou, S.L.; Peng, Y.C.; Lo, J.I.; Cheng, B.M. Identification of Nitrogen Defects in Diamond with Photoluminescence Excited in the 160-240 nm Region. Anal. Chem. 2012, 84, 9596-9600. [CrossRef]

198. Walker, J. Optical absorption and luminescence in diamond. Rep. Prog. Phys. 1979, 42, 1606-1659. [CrossRef]

199. Guan, S.; Peng, F.; Liang, H.; Fan, C.; Tan, L.; Wang, Z.; Zhang, Y.; Zhang, J.; Yu, H.; He, D. Fragmentation and stress diversification in diamond powder under high pressure. J. Appl. Phys. 2018, 124, 215902. [CrossRef] 
200. Kumar, A.; Lin, P.A.; Xue, A.; Hao, B.; Yap, Y.K.; Sankaran, R.M. Formation of nanodiamonds at near-ambient conditions via microplasma dissociation of ethanol vapour. Nat. Commun. 2013, 4, 2618. [CrossRef]

201. Tzeng, Y.-K.; Zhang, J.L.; Lu, H.; Ishiwata, H.; Dahl, J.; Carlson, R.M.K.; Yan, H.; Schreiner, P.R.; Vuckovic, J.; Shen, Z.-X.; et al. Vertical-Substrate MPCVD Epitaxial Nanodiamond Growth. Nano Lett. 2017, 17, 1489-1495. [CrossRef] [PubMed]

202. Nee, C.-N.; Lee, M.C.; Poh, H.S.; Yap, S.-S.; Tou, T.-Y.; Yap, S.-S. Plasma synthesis of nanodiamonds in ethanol. Compos. Part B 2019, 162, 162-166. [CrossRef]

203. Dolmatov, V.Y. Detonation-synthesis nanodiamonds: Synthesis, structure, properties and applications. Russ. Chem. Rev. 2007, 76, 339-360. [CrossRef]

204. Sun, L.; Wu, Q. Conversion of Graphite to diamond assisted by non-metallic catalysts under high pressure and high temperature: A review. High. Press Res. 1998, 16, 69-77. [CrossRef]

205. Tatsii, V.F.; Bochko, A.V.; Oleinik, G.S. Structure and Properties of Dalan Detonation Diamonds. Combust. Explos. Shock Waves 2009, 45, 95-103. [CrossRef]

206. Burkhard, G.; Tamura, H.; Tanabe, Y.; Sawaoka, A.B. Formation of diamond during passage of a shock wave in a copper/graphite powder:Formation process and numerical simulation. Appl. Phys. Lett. 1995, 66, 3131-3133. [CrossRef]

207. Mochalin, V.N.; Shenderova, O.; Ho, D.; Gogotsi, Y. The properties and applications of nanodiamonds. Nat. Nanotechnol. 2012, 7, 11-23. [CrossRef] [PubMed]

208. Dolmatov, V.Y. The Influence of Detonation Synthesis Conditions on the Yield of Condensed Carbon and Detonation Nanodiamond Through the Example of Using TNT-RDX Explosive Mixture. J. Superhard Mater. 2018, 40, 290-294. [CrossRef]

209. Mermoux, M.; Chang, S.; Girard, H.A.; Arnault, J.C. Raman spectroscopy study of detonation nanodiamond. Diam. Rel. Mater. 2018, 87, 248-260. [CrossRef]

210. Nee, C.-H.; Yap, S.-L.; Tou, S.-L.; Chang, H.-C.; Yap, S.-S. Direct synthesis of nanodiamonds by femtosecond laser irradiation of ethanol. Sci. Rep. 2016, 6, 33966. [CrossRef]

211. Ginés, L.; Mandal, S.; Ahmed, A.I.; Cheng, C.L.; Sow, M.; Williams, A. Positive zeta potential of nanodiamonds. Nanoscale 2017, 9 , 12549-12555. [CrossRef]

212. Turcheniuk, K.; Mochalin, V.N. Biomedical applications of nanodiamond. Nanotechnology 2017, 28, 252001. [CrossRef]

213. Neburkova, J.; Vavra, J.; Cigler, P. Coating nanodiamonds with biocompatible shells for applications inbiology and medicine. Curr. Opin. Solid State Mater. Sci. 2017, 21, 43-53. [CrossRef]

214. Chipaux, M.; van der Laan, K.J.; Hemelaar, S.R.; Hasani, M.; Zheng, T.; Schirhagl, R. Nanodiamonds and Their Applications in Cells. Small 2018, 14, 1704263. [CrossRef]

215. Martín, R.; Heydorn, P.C.; Heydorn, M.; Garcia, H. General strategy for high-density covalent functionalization of diamond nanoparticles using Fenton chemistry. Chem. Mater. 2009, 21, 4505-4514. [CrossRef]

216. Liang, Y.; Ozawa, M.; Krueger, A. A General Procedure to Functionalize Agglomerating Nanoparticles Demonstrated on Nanodiamond. ACS Nano 2009, 3, 2288-2296. [CrossRef]

217. Gibson, N.M.; Luo, T.-J.M.; Shenderova, O.; Koscheev, P.A.; Brenner, D.W. Electrostatically mediated adsorption by nanodiamond and nanocarbon particles. J. Nanopart Res. 2012, 14, 700. [CrossRef]

218. Petrova, N.; Zhukov, A.; Gareeva, F.; Koscheev, A.; Petrov, I.; Shenderova, O. Interpretation of electrokinetic measurements of nanodiamond particles. Diam. Rel. Mater. 2012, 30, 62-69. [CrossRef]

219. Osswald, S.; Yushin, G.; Mochalin, V.; Kucheyev, S.O.; Gogotsi, Y. Control of sp2/sp3 carbon ratio and surface chemistry of nanodiamond powders by selective oxidation in air. J. Am. Chem. Soc. 2006, 128, 11635-11642. [CrossRef]

220. Shenderova, O.; Koscheev, A.; Zaripov, N.; Petrov, I.; Skryabin, Y.; Detkov, P.; Turner, S. Surface Chemistry and Properties of Ozone-Purified Detonation Nanodiamonds. J. Phys. Chem. C 2011, 115, 9827-9837. [CrossRef]

221. Shuai, C.; Li, Y.; Wang, G.; Yang, W.; Peng, S.; Feng, P. Surface modification of nanodiamond: Toward the dispersion of reinforced phase in poly-l-lactic acid scaffoldss. Int. J. Biol. Macromol. 2019, 126, 1116-1124. [CrossRef]

222. Reina, G.; Gismondi, A.; Carcione, R.; Nanni, V.; Peruzzi, C.; Angjellari, M.; Chau, N.D.Q.; Canini, A.; Terranova, M.L. Oxidized and amino-functionalized nanodiamonds as shuttle for delivery of plant secondary metabolites: Interplay between chemical affinity and bioactivity. Appl. Surf. Sci. 2019, 470, 744-754. [CrossRef]

223. Zhang, X.; Fu, C.; Feng, L.; Ji, Y.; Tao, L.; Huang, Q.; Li, S.; Wei, Y. PEGylation and polyPEGylation of nanodiamond. Polymer 2012, 53, 3178-3184. [CrossRef]

224. Terada, D.; Sotoma, S.; Harada, Y.; Iragashi, R.; Shirakawa, M. One-pot synthesis of highly dispersible fluorescent nanodiamonds for bioconjugation. Bioconjug. Chem. 2018, 29, 2786-2792. [CrossRef]

225. Whitlow, J.; Pacelli, S.; Paul, A. Multifunctional nanodiamonds in regenerative medicine: Recent advances and future directions. J. Control. Release 2017, 261, 62-86. [CrossRef]

226. Reina, G.; Zhao, L.; Bianco, A.; Komatsu, N. Chemical Functionalization of Nanodiamonds: Opportunities and Challenges Ahead. Angew. Chem. Int. Ed. 2019, 58, 2-14. [CrossRef]

227. Kennedy, Z.C.; Barrett, C.A.; Warner, M.G. Direct Functionalization of an Acid-Terminated Nanodiamond with Azide: Enabling Access to 4-Substituted-1,2,3-Triazole-Functionalized Particles. Langmuir 2017, 33, 2790-2798. [CrossRef]

228. Tinwala, H.; Wairkar, S. Production, surface modification and biomedical applications of nanodiamonds: A sparkling tool for theranostics. Mater. Sci. Eng. C 2019, 97, 913-931. [CrossRef] 
229. Liu, H.; Reilly, S.; Herrnsdorf, J.; Xie, E.; Savitski, V.G.; Kemp, A.J.; Gu, E.; Dawson, M.D. Large radius of curvature micro-lenses on single crystal diamond for application in monolithic diamond Raman lasers. Diam. Rel. Mater. 2016, 65, 37-41. [CrossRef]

230. Woerner, E.; Wild, C.; Mueller-Sebert, W.; Koidl, P. CVD-diamond optical lenses. Diam. Rel. Mater. 2001, 10, 557-560. [CrossRef]

231. Shikata, S. Single crystal diamond wafers for high power electronics. Diam. Rel. Mater. 2016, 65, 168-175. [CrossRef]

232. Inspektor, A.; Oles, E.J.; Bauer, C.E. Theory and practice in diamond coated metal-cutting tools. Int. J. Refract. Met. Hard Mater. 1997, 15, 49-56. [CrossRef]

233. Li, J.; Li, W.; Zhang, Y.; Yang, X.; Chen, N.; Sun, Y.; Zhao, Y.; Fan, C.; Huang, Q. The Biocompatibility of Nanodiamonds and Their Application in Drug Delivery Systems. Theranostics 2012, 2, 302-312.

234. Juette, M.F.; Zhou, Z.; Altman, R.B.; Zheng, Q.; Blanchard, S.C. The bright future of single-molecule fluorescence imaging. Curr. Opin. Chem. Biol. 2014, 20, 103-111. [CrossRef]

235. Kusumi, A.; Tsunoyama, T.A.; Hirosawa, K.M.; Kasai, R.S.; Fujiwara, T.K. Tracking single molecules at work in living cells. Nat. Chem. Biol. 2014, 10, 524-532. [CrossRef]

236. Specht, E.A.; Braselmann, E.; Palmer, A.E. A Critical and Comparative Review of Fluorescent Tools for Live-Cell Imaging. Ann. Rev. Physiol. 2017, 79, 93-117. [CrossRef]

237. Degen, C.L.; Reinhard, F.; Cappellaro, P. Quantum sensing. Rev. Mod. Phys. 2017, 89, 035002. [CrossRef]

238. Aharonovich, I.; Neu, E. Diamond Nanophotonics. Adv. Opt. Mater. 2014, 2, 911-928. [CrossRef]

239. Ermakova, A.; Pramanik, G.; Cai, J.-M.; Algara-Siller, G.; Kaiser, U.; Weil, T.; Tzeng, Y.-K.; Chang, H.C.; Naydenov, B.; Jelezko, F. Detection of a Few Metallo-Protein Molecules Using Color Centers in Nanodiamonds. Nano Lett. 2013, 13, 3305-3309. [CrossRef]

240. Schroder, T.; Schell, A.W.; Kewes, G.; Aichele, T.; Benson, O. Fiber-Integrated Diamond-Based Single Photon Source. Nano Lett. 2011, 11, 198-202. [CrossRef]

241. Liu, Y.-Y.; Chang, B.-M.; Chang, H.-C. Nanodiamond-enabled biomedical imaging. Nanomedicine 2020, 15, 1599-1616. [CrossRef]

242. Wrachtrup, J.; Jelezko, F. Processing quantum information in diamond. J. Phys. Condens Matter 2006, 18, S807-S824. [CrossRef]

243. Hensen, B.; Bernien, H.; Dréau, A.E.; Reiserer, A.; Kalb, N.; Blok, M.S.; Ruitenberg, J.; Vermeulen, R.F.L.; Schouten, R.N.; Abellán, C.; et al. Loophole-free Bell inequality violation using electron spins separated by 1.3 kilometres. Nature 2015, 526, 682-686. [CrossRef]

244. Ajoy, A.; Bissbort, U.; Lukin, M.D.; Walsworth, R.L.; Cappellaro, P. Atomic-Scale Nuclear Spin Imaging Using Quantum-Assisted Sensors in Diamond. Phys. Rev. X 2015, 5. [CrossRef]

245. Bucher, D.B.; Glenn, D.R.; Park, H.; Lukin, M.D.; Walworth, R.L. Hyperpolarization-Enhanced NMR Spectroscopy with Femtomole Sensitivity Using Quantum Defects in Diamond. Phys. Rev. X 2020, 10, 021053. [CrossRef]

246. Dolde, F.; Fedder, H.; Doherty, W.; Nöbauer, M.W.; Rempp, F.; Wolf, T.; Reinhard, F.; Hollenberg, L.C.L.; Jelezko, F.; Wrachtrup, J. Electric field sensing using single diamond spins. Nat. Phys. 2011, 7, 459-463. [CrossRef]

247. Blok, M.; Kobrin, B.; Jarmola, A.; Hsieh, S.; Zu, C.; Figueroa, N.L.; Acosta, V.M.; Minguzzi, J.; Maze, J.R.; Budker, D.; et al. Optically Enhanced Electric Field Sensing using Nitrogen-Vacancy Ensembles. arXiv 2020, arXiv:2004.02886.

248. Hsieh, S.; Bhattacharyya, P.; Zu, C.; Mittiga, T.; Smart, T.J.; Machado, F.; Kobrin, B.; Höhn, T.O. Imaging stress and magnetism at high pressures using a nanoscale quantum sensor. Science 2019, 366, 1349-1354. [CrossRef]

249. Kehayias, P.; Turner, J.; Trubko, R.; Schloss, J.M.; Hart, C.A.; Wesson, M.; Glenn, D.R.; Walsworth, R.L. Imaging crystal stress in diamond using ensembles of nitrogen-vacancy centers. Phys. Rev. B 2019, 100, 174103. [CrossRef]

250. Childress, L.; Gurudev Dutt, M.V.; Taylor, J.M.; Zibrov, A.S.; Jelezko, F.; Wrachtrup, J.; Hemmer, P.R.; Lukin, M.D. Coherent Dynamics of Coupled Electron and Nuclear Spin Qubits in Diamond. Science 2006, 314, 281-285. [CrossRef]

251. Becker, J.N.; Neu, E. The silicon vacancy center in diamond. Semicond Semimet. 2020, 103, 201-235.

252. Nguyen, C.T.; Evans, R.E.; Sipahigil, A.; Bhaskar, M.K.; Sukachev, D.D.; Agafonov, V.N.; Davydov, V.A.; Kulikova, L.F.; Jelezko, F.; Lukin, M.D. All-optical nanoscale thermometry with silicon-vacancy centers in diamond. Appl. Phys. Lett. 2018, 112, 203102. [CrossRef]

253. Okabe, K.; Sakaguchi, R.; Kiyonaka, S. Intracellular thermometry with fluorescent sensors for thermal biology. Pflüg. Arch. Eur. J. Physiol. 2018, 470, 717-731. [CrossRef]

254. Merson, T.D.; Castelletto, S.; Aharonovich, T.; Kilpatrick, T.J.; Turnley, A.M. Nanodiamonds with silicon vacancy defects for nontoxic photostable fluorescent labeling of neural precursor cells. Opt. Lett. 2013, 38, 4170-4173. [CrossRef]

255. Silani, Y.; Hubert, F.; Acosta, V.M. Stimulated Emission Depletion Microscopy with Diamond Silicon Vacancy Centers. ACS Photon. 2019, 6, 1577-1582. [CrossRef]

256. Prabhakar, N.; Perula, M.; Koho, S.; Deguchi, T.; Nareoja, T.; Chang, H.-C.; Rosenholm, J.M.; Hanningen, P. STED-TEM Correlative Microscopy Leveraging Nanodiamonds as Intracellular Dual-Contrast Markers. Small 2018, 14, 1701807. [CrossRef]

257. Kuo, Y.; Hsu, T.-Y.; Wu, Y.-C.; Chang, H.-C. Fluorescent nanodiamond as a probe for the intercellular transport of proteins in vivo. Biomaterials 2013, 34, 8352-8360. [CrossRef]

258. Fang, C.Y.; Vaijayanthimala, V.; Cheng, C.A.; Yeh, S.H.; Chang, C.F.; Li, H.C.; Chang, H.C. The exocytosis of fluorescent nanodiamond and its use as a long-term cell tracker. Small 2011, 7, 3363-3370. [CrossRef]

259. Chow, E.K.; Zhang, X.Q.; Chen, M.; Lam, R.; Robinson, E.; Huang, H.; Schaffer, D.; Osawa, E.; Goga, A.; Ho, D. Nanodiamond therapeutic delivery agents mediate enhanced chemoresistant tumor treatment. Sci. Transl. Med. 2011, 3, 73ra21. [CrossRef] 
260. ̌̌ehoř, I.; Šlegerová, J.; Havlík, J.; Raabová, H.; Hývl, J.; Muchová, E.; Cígler, P. Nanodiamonds: Behavior in Biological Systems and Emerging Bioapplications. In Carbon Nanomaterials for Biomedical Applications; Springer Series in Biomaterials Science and Engineering; Springer International Publishing: Cham, Switzerland, 2016; p. 11.

261. Bortolucci Simioni, N.; Almeida Silva, T.; Gabriel Oliveira, G.; Fatibello-Filho, O. A nanodiamond-based electrochemical sensor for the determination of pyrazinamide antibiotic. Sens. Actuators B 2017, 250, 315-323. [CrossRef]

262. Jiang, L.; Santiago, I.; Foord, J. A comparative study of fouling-free nanodiamond and nanocarbon electrochemical sensors for sensitive bisphenol A detection. Carbon 2021, 174, 390-395. [CrossRef]

263. Wong, A.; Ferreira, P.A.; Santos, A.M.; Cincotto, F.H.; Silva, R.A.B.; Sotomayor, M.D.P.T. A new electrochemical sensor based on eco-friendly chemistry for the simultaneous determination of toxic trace elements. Microchem. J. 2020, 158, 105292. [CrossRef]

264. Kumar, S.; Nehra, M.; Kedia, D.; Dilbaghi, N.; Tankeshwar, K.; Kim, K.-H. Nanodiamonds: Emerging face of future nanotechnology. Carbon 2019, 143, 678-699. [CrossRef]

265. Lim, S.Y.; Shen, W.; Gao, Z. Carbon quantum dots and their applications. Chem. Soc. Rev. 2015, 44, 362-381. [CrossRef]

266. Yang, S.; Sun, J.; Li, X.; Zhou, W.; Wang, Z.; He, P.; Ding, G.; Xie, X.; Kang, Z.; Jiang, M. Large scale fabrication of heavy doped carbon quantum dots with tunable photoluminescence and sensitive fluorescence detection. J. Mater. Chem. A 2014, 2, 8660-8667. [CrossRef]

267. Zhang, M.; Bai, L.; Shang, W.; Xie, W.; Ma, H.; Fu, Y.; Fang, D.; Sun, H.; Fan, L.; Han, M.; et al. Facile synthesis of water-soluble, highly fluorescent graphene quantum dots as a robust biological label for stem cells. J. Mater. Chem. 2012, 22, $7461-7467$. [CrossRef]

268. Yatom, B.; Bak, J.; Khrabryi, A.; Raitses, Y. Detection of nanoparticles in carbon arc discharge with laser-induced incandescence. Carbon 2017, 117, 154-162. [CrossRef]

269. Xu, X.Y.; Ray, T.; Gu, Y.L.; Ploehn, H.J.; Gearheart, L.; Raker, K. Electrophoretic analysis and purification of fluorescent single walled carbon nanotube fragments. J. Am. Chem. Soc. 2004, 126, 12736-12737. [CrossRef]

270. Kang, S.; Jeong, Y.K.; Son, Y.; Kim, W.R.; Lee, B.; Jung, K.H.; Kim, K.M. Pulsed laser ablation based synthetic route for nitrogendoped graphene quantum dots using graphite flakes. Appl. Surf. Sci. 2020, 506, 144998. [CrossRef]

271. Donate-Buendia, C.; Torres-Mendieta, R.; Pyatenko, A.; Falomir, E.; Fernandez-Alonso, M.; Minguez-Vega, G. Fabrication by laser irradiation in a continuous flow jet of carbon quantum dots for fluorescence imaging. ACS Omega 2018, 3, 2735-2742. [CrossRef]

272. Liu, H.P.; Ye, T.; Mao, C.D. Fluorescent carbon nanoparticles derived from candle soot. Angew. Chem. Int. Ed. 2007, 46, 6473-6475. [CrossRef]

273. Schwenke, A.M.; Hoeppener, S.; Schubert, U.S. Synthesis and modification of carbon nanomaterials utilizing microwave heating. Adv. Mater. 2015, 27, 4113-4141. [CrossRef]

274. Wei, W.; Xu, C.; Wu, L.; Wang, J.; Ren, J.; Qu, X. Non-Enzymatic-Browning-Reaction: A Versatile Route for Production of Nitrogen-Doped Carbon Dots with Tunable Multicolor Luminescent Display. Sci. Rep. 2014, 4, 3564/1-3564/7. [CrossRef]

275. Wang, G.; Guo, G.; Liu, Z.; Zheng, X.; Xu, A.; Yang, S.; Ding, G. Facile and highly effective synthesis of controllable lattice sulfur-doped graphene quantum dots via hydrothermal treatment of durian. ACS Appl. Mater. Interf. 2018, 10, 5750-5759. [CrossRef]

276. Liu, J.; Li, D.; Zhang, K.; Yang, M.; Sun, H.; Yang, B. One-step hydrothermal synthesis of nitrogen-doped conjugated carbonized polymer dots with $31 \%$ efficient red emission for in vivo imaging. Small 2018, 14, 1703919. [CrossRef]

277. Li, H.; He, X.; Liu, Y.; Huang, H.; Lian, S.; Lee, S.-T.; Kang, Z. One-step ultrasonic synthesis of water-soluble carbon nanoparticles with excellent photoluminescent properties. Carbon 2011, 49, 605-609. [CrossRef]

278. Reckmeier, C.J.; Schneider, J.; Susha, A.S.; Rogach, A.L. Luminescent colloidal carbon dots: Optical properties and effects of doping. Opt. Express 2016, 24, A312-A340. [CrossRef]

279. Chandra, S.; Pathan, S.H.; Mitra, S.; Modha, B.H.; Goswami, A.; Pramink, P. Tuning of photoluminescence on different surface functionalized carbon quantum dots. RSC Adv. 2012, 2, 3602-3606. [CrossRef]

280. Arcudi, F.; Dordevic, L.; Prato, M. Design, Synthesis, and Functionalization Strategies of Tailored Carbon Nanodots. Acc. Chem. Res. 2019, 52, 2070-2079. [CrossRef]

281. Li, H.; Kang, Z.; Liu, Y.; Lee, S.T. Carbon nanodots: Synthesis, properties and applications. J. Mater. Chem. 2012, 22, 24230-24253. [CrossRef]

282. Tajik, S.; Dourandish, Z.; Zhang, K.; Beitollahi, H.; Van Le, Q.; Jang, H.W.; Shokouhimehr, M. Carbon and graphene quantum dots: A review on syntheses, characterization, biological and sensing applications for neurotransmitter determination. RSC Adv. 2020, 10, 15406-15429. [CrossRef]

283. Bourlinos, A.B.; Stassinopoulos, A.; Anglos, D.; Zboril, R.; Karakassides, M.; Giannelis, E.P. Surface functionalized carbogenic quantum dots. Small 2008, 4, 455-458. [CrossRef]

284. Ding, C.; Zhu, A.; Tian, Y. Functional surface engineering of C-dots for fluorescent biosensing and in vivo bioimaging. Acc. Chem. Res. 2014, 47, 20-30. [CrossRef]

285. Zhu, S.; Song, Y.; Zhao, X.; Shao, J.; Zhang, J.; Yang, B. The Photoluminescence Mechanism in Carbon Dots (Graphene Quantum Dots, Carbon Nanodots, and Polymer Dots): Current State and Future Perspective. Nano Res. 2015, 8, 355-381. [CrossRef]

286. Carbonaro, C.M.; Corpino, R.; Salis, M.; Mocci, F.; Thakkar, S.V.; Olla, C.; Ricci, P.C. On the Emission Properties of Carbon Dots: Reviewing Data and Discussing Models. C J. Carbon Res. 2019, 5, 60. [CrossRef] 
287. Zhang, Y.; He, J. Facile Synthesis of S, N Co-Doped Carbon Dots and Investigation of their Photoluminescence Properties. Phys. Chem. Chem. Phys. 2015, 17, 20154-20159. [CrossRef]

288. Sun, Y.-P.; Zhou, B.; Lin, Y.; Wang, W.; Shiral Fernando, K.A.; Pathak, P.; Meziani, M.J.; Harruff, B.; Wang, X.; Wang, H.; et al. Quantum-Sized Carbon Dots for Bright and Colorful Photoluminescence. J. Am. Chem. Soc. 2006, 128, 7756-7757. [CrossRef] [PubMed]

289. Li, H.; He, X.; Kang, Z.; Huang, H.; Liu, Y.; Liu, J.; Lian, S.; Tsang, C.H.A.; Yang, X.; Lee, S.-T. Water-Soluble Fluorescent Carbon Quantum Dots and Photocatalyst Design. Angew. Chem. Int. Ed. 2010, 49, 4430-4434. [CrossRef] [PubMed]

290. Zhang, R.Q.; Bertran, E.; Lee, S.T. Size dependence of energy gaps in small carbon clusters: The origin of broadband luminescence. Diam. Rel. Mater. 1998, 7, 1663-1668. [CrossRef]

291. Sharma, A.; Gadly, T.; Neogy, S.; Gosh, S.K.; Kumbhakar, M. Molecular Origin and Self-Assembly of Fluorescent Carbon Nanodots in Polar Solvents. J. Phys. Chem. Lett. 2017, 8, 1044-1052. [CrossRef]

292. Carbonaro, C.M.; Chiriu, D.; Stagi, L.; Casula, M.F.; Takkar, S.V.; Malfatti, L.; Suzuki, K.; Ricci, P.C.; Corpino, R. Carbon Dots in Water and Mesoporous Matrix: Chasing the Origin of their Photoluminescence. J. Phys. Chem. C 2018, 122, 25638-25650. [CrossRef]

293. Yeh, T.-F.; Huang, W.-L.; Chung, C.-J.; Chiang, I.-T.; Chen, L.-C.; Chang, H.-Y.; Cheng, C.; Chen, S.-J.; Teng, H. Elucidating Quantum Confinement in Graphene Oxide Dots Based On Excitation-Wavelength-Independent Photoluminescence. J. Phys. Chem. Lett. 2016, 2087-2092. [CrossRef]

294. Lakowicz, J.R. Principles of Fluorescence Spectroscopy, 3rd ed.; Springer: Singapore, 2006.

295. Guo, Y.; Zhang, S.; Yang, Y.; Chen, X.; Zhang, M. Fluorescent carbon nanoparticles for the fluorescent detection of metal ions. Biosens. Bioelectron 2015, 63, 61-71. [CrossRef]

296. Zhu, S.; Meng, Q.; Wang, L.; Zhang, J.; Song, Y.; Jin, H.; Zhang, K.; Sun, H.; Wang, H.; Yang, B. Highly photoluminescent carbon dots for multicolor patterning, sensors, and bioimaging. Angew. Chem. Int. Ed. 2013, 125, 3953-3957. [CrossRef]

297. Cui, X.; Zhu, L.; Wu, J.; Hou, Y.; Wang, P.; Wang, Z.; Yang, M. A fluorescent biosensor based on carbon dots-labeled oligodeoxyribonucleotide and graphene oxide for mercury (II) detection. Biosens. Bioelectron. 2015, 63, 506-512. [CrossRef] [PubMed]

298. Zhong, J.; Yang, X.; Trinchi, A.; Hardin, S.; Cole, I.; Zhu, Y.; Li, C.; Muster, T.; Wei, G. Carbon dots as fluorescent probes for "off-on" detection of $\mathrm{Cu} 2+$ and 1-cysteine in aqueous solution. Biosens. Bioelectron. 2014, 51, 330-335. [CrossRef] [PubMed]

299. Cai, F.; Liu, X.; Liu, S.; Liu, H.; Huang, Y. A simple one-pot synthesis of highly fluorescent nitrogen-doped graphene quantum dots for the detection of Cr (VI) in aqueous media. RSC Adv. 2014, 4, 52016-52022. [CrossRef]

300. Ravi, S.; Jayaraj, M.K. Sustainable carbon dots as "turn-off" fluorescence sensor for highly sensitive Pb ${ }^{2+}$ detection. Emerg. Mater. 2020, 3, 51-56. [CrossRef]

301. Liao, J.; Cheng, Z.; Zhou, L. Nitrogen-Doping Enhanced Fluorescent Carbon Dots: Green Synthesis and Their Applications for Bioimaging and Label-Free Detection of $\mathrm{Au}^{3+}$ Ions. ACS Sustain. Chem. Eng. 2016, 4, 3053-3061. [CrossRef]

302. Gao, Z.; Wang, S.; Xu, Z.; Liu, J.; Huang, Y.; Hu, S.; Ren, X. Synthesis of novel cationic carbon dots and application to quantitative detection of $\mathrm{K}+$ in human serum samples. New J. Chem. 2019, 43, 17937-17940. [CrossRef]

303. Gao, X.; Lu, Y.; Zhang, R.; He, S.; Ju, J.; Liu, M.; Lia, L.; Chen, W. One-pot synthesis of carbon nanodots for fluorescence turn-on detection of Ag+ based on the Ag+-induced enhancement of fluorescence. J. Mater. Chem. C 2015, 3, 2302-2309. [CrossRef]

304. Hou, X.; Zeng, F.; Du, F.; Wu, S. Carbon-dot-based fluorescent turn-on sensor for selectively detecting sulfide anions in totally aqueous media and imaging inside live cells. Nanotechnology 2013, 24, 335502. [CrossRef]

305. Zhu, A.; Luo, Z.; Ding, C.; Li, B.; Zhou, S.; Wang, R.; Tian, Y. A two-photon "turn-on" fluorescent probe based on carbon nanodots for imaging and selective biosensing of hydrogen sulfide in live cells and tissues. Analyst 2014, 139, 1945-1952. [CrossRef] [PubMed]

306. Dong, Y.; Wang, R.; Tian, W.; Chi, Y.; Chen, G. “Turn-on" fluorescent detection of cyanide based on polyamine-functionalized carbon quantum dots. RSC Adv. 2014, 4, 3701-3705. [CrossRef]

307. Zhao, H.X.; Liu, L.Q.; Wang, Y.; Zhao, X.J.; Huang, C.Z. Highly selective detection of phosphate in very complicated matrixes with an off-on fluorescent probe of europium-adjusted carbon dots. Chem. Commun. 2011, 47, 2604-2606. [CrossRef] [PubMed]

308. Zhao, D.; Chen, C.; Lu, L.; Yang, F.; Yang, X. A dual-mode colorimetric and fluorometric "light on" sensor for thiocyanate based on fluorescent carbon dots and unmodified gold nanoparticles. Analyst 2015, 140, 8157-8164. [CrossRef]

309. Sun, X.; Wang, Y.; Lei, Y. Fluorescence based explosive detection: From mechanisms to sensory materials. Chem. Soc. Rev. 2015, 44, 8019-8061. [CrossRef]

310. Wang, D.; Wang, Z.; Zhan, Q.; Pu, Y.; Wang, J.-X.; Foster, N.R.; Dai, L. Facile and Scalable Preparation of Fluorescent Carbon Dots for Multifunctional Applications. Engineering 2017, 3, 401-408. [CrossRef]

311. Huang, Q.; Hu, S.; Zhang, H.; Chen, J.; He, Y.; Li, F.; Lin, Y. Carbon dots and chitosan composite film based biosensor for the sensitive and selective determination of dopamine. Analyst 2013, 138, 5417-5423. [CrossRef] [PubMed]

312. Hu, S.; Huang, Q.; Lin, Y.; Wei, C.; Zhang, H.; Zhang, W.; Hao, A. Reduced graphene oxide-carbon dots composite as an enhanced material for electrochemical determination of dopamine. Electrochim. Acta 2014, 130, 805-809. [CrossRef]

313. Wang, L.; Chen, X.; Liu, C.; Yang, W. Non-enzymatic acetylcholine electrochemical biosensor based on flower-like NiAl layered double hydroxides decorated with carbon dots. Sens. Actuators B 2016, 233, 199-205. [CrossRef]

314. Yola, M.L.; Atar, N. Development of molecular imprinted sensor including graphitic carbon nitride/N- doped carbon dots composite for novel recognition of epinephrine. Compos. B 2019, 175, 107113. [CrossRef] 
315. Qu, Z.-B.; Zhou, X.; Gu, L.; Lan, R.; Sun, D.; Yu, D.; Shi, G. Boronic acid function-alized graphene quantum dots as a fluorescent probe for selective and sensitive glucose determination in microdialysate. Chem. Commun. 2013, 49, 9830-9832. [CrossRef]

316. Wang, Q.; Liu, X.; Zhang, L.; Lv, Y. Microwave-assisted synthesis of carbon nanodots through an eggshell membrane and their fluorescent application. Analyst 2012, 137, 5392-5397. [CrossRef]

317. Jiang, K.; Sun, S.; Zhang, L.; Wang, Y.; Cai, C.; Lin, H. Bright-yellow-emissive N-Doped carbon dots: Preparation, cellular imaging, and bifunctional sensing. ACS Appl. Mater. Interf. 2015, 7, 23231-23238. [CrossRef]

318. Niu, J.; Gao, H. Synthesis and drug detection performance of nitrogen-doped carbon dots. J. Lumin. 2014, 149, 159-162. [CrossRef]

319. Wang, J.; Wei, J.; Su, S.; Qiu, J. Novel fluorescence resonance energy transfer optical sensors for vitamin B12 detection using thermally reduced carbon dots. New J. Chem. 2015, 39, 501-507. [CrossRef]

320. Wang, C.-I.; Wu, W.-C.; Periasamy, A.P.; Chang, H.-T. Electrochemical synthesis of photoluminescent carbon nanodots from glycine for highly sensitive detection of hemoglobin. Green Chem. 2015, 16, 2509-2514. [CrossRef]

321. Bai, W.; Zheng, H.; Long, Y.; Mao, X.; Gao, M.; Zhang, L. A carbon dots-based fluorescence turn-on method for DNA determination. Anal. Sci. 2011, 27, 243-246. [CrossRef] [PubMed]

322. Long, C.; Jiang, Z.; Shangguan, J.; Qing, T.; Zhang, P.; Feng, B. Applications of carbon dots in environmental pollution control: A review. Chem. Eng. J. 2021, 405, 126848. [CrossRef]

323. Ruiz-Palomero, C.; Soriano, M.L.; Benitez-Martinez, S.; Valcarcel, M. Photoluminescent sensing hydrogel platform based on the combination of nanocellulose and S, N-codoped graphene quantum dots. Sens. Actuators B 2017, 245, 946-953. [CrossRef]

324. Sun, X.; Lei, Y. Fluorescent carbon dots and their sensing applications. Trends Anal. Chem. 2017, 89, 163-180. [CrossRef]

325. Huang, M.; Liang, X.; Zhang, Z.; Wang, J.; Fei, Y.; Ma, J.; Qu, S.; Mi, L. Carbon Dots for Intracellular pH Sensing with Fluorescence Lifetime Imaging Microscopy. Nanomaterials 2020, 10, 604. [CrossRef]

326. Wang, N.; Zheng, A.Q.; Liu, X.; Chen, J.J.; Yang, T.; Chen, M.; Wang, J.H. Deep Eutectic Solvent-Assisted Preparation of Nitrogen/Chloride-Doped Carbon Dots for Intracellular Biological Sensing and Live Cell Imaging. ACS Appl. Mater. Interf. 2018, 10, 7901-7909. [CrossRef]

327. Hamd-Ghadareh, S.; Salimi, A.; Fathi, F.; Bahrami, S. An amplified comparative fluorescence resonance energy transfer immunosensing of CA125 tumor marker and ovarian cancer cells using green and economic carbon dots for bio-applications in labeling, imaging and sensing. Biosens. Bioelectron. 2017, 96, 308-316. [CrossRef]

328. Zhou, N.; Hao, Z.Y.; Zhao, X.H.; Maharjan, S.; Zhu, S.J.; Song, Y.B.; Yang, B.; Lu, L.J. A novel fluorescent retrograde neural tracer:cholera toxin B conjugated carbon dots. Nanoscale 2015, 7, 15635-15642. [CrossRef]

329. Kang, Y.F.; Fang, Y.W.; Li, Y.H.; Li, W.; Yin, X.B. Nucleus-staining with biomolecule-mimicking nitrogen-doped carbon dots prepared by a fast neutralization heat strategy. Chem. Commun. 2015, 51, 16956-16959. [CrossRef]

330. Hua, X.W.; Bao, Y.W.; Chen, Z.; Wu, F.G. Carbon quantum dots with intrinsic mitochondrial targeting ability for mitochondriabased theranostics. Nanoscale 2017, 9, 10948-10960. [CrossRef] [PubMed]

331. Zhang, Q.Q.; Yang, T.; Li, R.S.; Zou, H.Y.; Li, Y.F.; Guo, J.; Liu, X.D.; Huang, C.Z. A functional preservation strategy for the production of highly photoluminescent emerald carbon dots for lysosome targeting and lysosomal pH imaging. Nanoscale 2018, 10, 14705-14711. [CrossRef]

332. Tao, H.Q.; Yang, K.; Ma, Z.; Wan, J.M.; Zhang, Y.J.; Kang, Z.H.; Liu, Z. In vivo NIR fluorescence imaging, biodistribution, and toxicology of photoluminescent carbon dots produced from carbon nanotubes and graphite. Small 2012, 8, 281-290. [CrossRef] [PubMed]

333. Wang, Y.; Meng, Y.; Wang, S.S.; Li, C.Y.; Shi, W.; Chen, J.; Wang, J.X.; Huang, R.Q. Direct Solvent-Derived Polymer-Coated Nitrogen-Doped Carbon Nanodots with High Water Solubility for Targeted Fluorescence Imaging of Glioma. Small 2015, 11, 3575-3581. [CrossRef]

334. Licciardello, N.; Hunoldt, S.; Bergman, R.; Singh, G.; Mamat, C.; Faramus, A.; Ddungu, J.L.Z.; Silvestrini, S.; Maggini, M.; De Cola, L.; et al. Biodistribution studies of ultrasmall silicon nanoparticles and carbon dots in experimental rats and tumor mice. Nanoscale 2018, 10, 9880-9891. [CrossRef] [PubMed]

335. Feng, T.; Ai, X.Z.; An, G.H.; Yang, P.P.; Zhao, Y.L. Charge-Convertible Carbon Dots for Imaging-Guided Drug Delivery with Enhanced in Vivo Cancer Therapeutic Efficiency. ACS Nano 2016, 10, 4410-4420. [CrossRef]

336. Gao, N.; Yang, W.; Nie, H.L.; Gong, Y.Q.; Jing, J.; Gao, L.J.; Zhang, X.L. Turn-on theranostic fluorescent nanoprobe by electrostatic self-assembly of carbon dots with doxorubicin for targeted cancer cell imaging, in vivo hyaluronidase analysis, and targeted drug delivery. Biosens. Bioelectron. 2017, 96, 300-307. [CrossRef]

337. Zheng, D.W.; Li, B.; Li, C.X.; Fan, J.X.; Lei, Q.; Li, C.; Xu, Z.S.; Zhang, X.Z. Carbon-Dot-Decorated Carbon Nitride Nanoparticles for Enhanced Photodynamic Therapy against Hypoxic Tumor via Water Splitting. ACS Nano 2016, 10, 8715-8722. [CrossRef]

338. Zhou, Y.; Liyanage, P.Y.; Devadoss, D.; Rios Guevara, L.R.; Cheng, L.; Graham, R.M.; Chand, H.S.; Al-Youbi, A.; Bashammakh, A.S.; El-Shahawi, M.S.; et al. Nontoxic amphiphilic carbon dots as promising drug nanocarriers across the blood-brain barrier and inhibitors of $\hat{\mathrm{I}}^{2}$-amyloid. Nanoscale 2019, 11, 22387-22397. [CrossRef]

339. Borisova, T.; Nazarova, A.; Dekaliuk, M.; Krisanova, N.; Pozdnyakova, N.; Borysov, A.; Sivko, R.; Demchenko, A.P. Neuromodulatory properties of fluorescent carbon dots: Effect on exocytotic release, uptake and ambient level of glutamate and GABA in brain nerve terminals. Int. J. Biochem. Cell Biol. 2015, 59, 203-215. [CrossRef]

340. Yu, M.-F.; Files, B.S.; Arepalli, S.; Ruoff, R.S. Tensile Loading of Ropes of Single Wall Carbon Nanotubes and their Mechanical Properties. Phys. Rev. Lett. 2000, 84, 5552-5555. [CrossRef] 
341. Joselevich, E.; Lieber, C.M. Vectorial Growth of Metallic and Semiconducting Single-Wall Carbon Nanotubes. Nano Lett. 2002, 2, 1137-1141. [CrossRef]

342. Kalamkarov, A.L.; Georgiades, A.V.; Rokkam, S.K.; Veedu, V.P.; Ghasemi-Nejhad, M.N. Analytical and numerical techniques to predict carbon nanotubes properties. Int. J. Sol. Struct. 2006, 43, 6832-6854. [CrossRef]

343. Ebbesen, T.W.; Lezec, H.J.; Hiura, H.; Bennett, J.W.; Ghaemi, H.F.; Thio, T. Electrical conductivity of individual carbon nanotubes Nature 1996, 382, 54-56. [CrossRef]

344. Li, Q.W.; Li, Y.; Zhang, X.F.; Chikkannanavar, S.B.; Zhao, Y.H.; Dangelewicz, A.M.; Zheng, L.X.; Doorn, S.K.; Jia, Q.X.; Peterson, D.E.; et al. Structure-Dependent Electrical Properties of Carbon Nanotube Fibers. Adv. Mater. 2007, 19, 3358-3363. [CrossRef]

345. Pop, E.; Mann, D.; Wang, Q.; Goodson, K.; Dai, H. Thermal Conductance of an Individual Single-Wall Carbon Nanotube above Room Temperature. Nano Lett. 2006, 6, 96-100. [CrossRef]

346. Kim, P.; Shi, L.; Majumdar, A.; McEuen, P.L. Thermal Transport Measurements of Individual Multiwalled Nanotubes. Phys. Rev. Lett. 2001, 87, 215502. [CrossRef] [PubMed]

347. Peigney, A.; Laurent, C.; Flahaut, E.; Bacsa, R.R.; Rousset, A. Specific surface area of carbon nanotubes and bundles of carbon nanotubes. Carbon 2001, 39, 507-514. [CrossRef]

348. Cinke, M.; Li, J.; Chen, B.; Cassell, A.; Delzeit, L.; Han, J.; Meyyappan, M. Pore structure of raw and purified HiPco single-walled carbon nanotubes. Chem. Phys. Lett. 2002, 365, 69-74. [CrossRef]

349. Priyanka, S.; Neelesh Kumar, M.; Keerti, J.; Jain, N.K. Biomedical Applications of Carbon Nanotubes: A Critical Review. Curr. Drug Deliv. 2016, 13, 796-817. [CrossRef]

350. Deb, J.; Debolina, P.; Sarkar, U.; Ayers, P.W. Characterizing the sensitivity of bonds to the curvature of carbon nanotubes. J. Mol. Model. 2018, 24, 249. [CrossRef]

351. Iijima, S. Helical microtubules of graphitic carbon. Nature 1991, 354, 56-58. [CrossRef]

352. Prasek, J.; Drbohlavova, J.; Chomoucka, J.; Hubalek, J.; Jasek, O.; Adamc, V.; Kizek, R. Methods for carbon nanotubes synthesisReview. J. Mater. Chem. 2011, 21, 15872-15884. [CrossRef]

353. Eatemadi, A.; Daraee, H.; Karimkhanloo, H.; Kouhi, M.; Zarghami, N.; Akbarzadeh, A.; Abasi, M.; Henifehpour, Y.; Joo, S.W. Carbon nanotubes: Properties, synthesis, purification, and medical applications. Nanoscale Res. Lett. 2014, 9, 393. [CrossRef]

354. Arora, N.; Sharma, N.N. Arc discharge synthesis of carbon nanotubes: Comprehensive review. Diam. Rel. Mater. 2014, 50, 135-150. [CrossRef]

355. Wang, M.; Zhao, X.L.; Ohkochi, M.; Ando, Y. Carbon Nanotubes Grown on the Surface of Cathode Deposit by Arc Discharge. Fuller. Sci. Technol. 1996, 4, 1027-1039. [CrossRef]

356. Shimotani, K.; Anazawa, K.; Watanabe, H.; Shimizu, M. New synthesis of multi-walled carbon nanotubes using an arc discharge technique under organic molecular atmospheres. Appl. Phys. A 2001, 73, 451-454.

357. Jiang, Y.; Wang, H.; Shang, X.F.; Li, H.; Wang, M. Influence of $\mathrm{NH}_{3}$ atmosphere on the growth and structures of carbon nanotubes synthesized by the arc-discharge method. Inorg. Mater. 2009, 45, 1237-1239.

358. Parkansky, N.; Boxman, R.L.; Alterkop, B.; Zontag, I.; Lereah, Y.; Barkay, Z. Single-pulse arc production of carbon nanotubes in ambient air. J. Phys. D 2004, 37, 2715-2719. [CrossRef]

359. Ebbesen, T.W.; Ajayan, P.M. Large-scale synthesis of carbon nanotubes. Nature 1992, 358, 220-222. [CrossRef]

360. Bethune, D.S.; Kiang, C.H.; Devries, M.S.; Gorman, G.; Savoy, R.; Vazquez, J.; Beyers, R. Cobalt-catalysed growth of carbon nanotubes with single-atomic-layer walls. Nature 1993, 363, 605-607. [CrossRef]

361. Seraphin, S.; Zhou, D.; Jiao, J.; Minke, M.A.; Wang, S.; Yadav, T.; Withers, J.C. Catalytic role of nickel, palladium, and platinum in the formation of carbon nanoclusters. Chem. Phys. Lett. 1944, 217, 191-198. [CrossRef]

362. Rahman, G.; Najaf, Z.; Mehmood, A.; Bilal, S.; ul Haq Ali Shah, A.; Mian, S.A.; Ali, G. An Overview of the Recent Progress in the Synthesis and Applications of Carbon Nanotubes. C J. Carbon Res. 2019, 5, 3. [CrossRef]

363. Chrzanowska, J.; Hoffman, J.; Malolepszy, A.; Mazurkiewicz, M.; Kowalewski, T.A.; Szymanski, Z.; Stobinski, L. Synthesis of carbon nanotubes by the laser ablation method: Effect of laser wavelength. Phys. Stat. Sol. B 2015, 252, 1860-1867. [CrossRef]

364. Guo, T.; Nikolaev, P.; Thess, A.; Colbert, D.T.; Smalley, R.E. Catalytic growth of single-walled manotubes by laser vaporization. Chem. Phys. Lett. 1995, 243, 49-54. [CrossRef]

365. Das, R.; Shahnavaz, Z.; Ali, E.; Islam, M.M.; Abd Hamid, S.B. Can We Optimize Arc Discharge and Laser Ablation for WellControlled Carbon Nanotube Synthesis? Nanoscale Res. Lett. 2016, 11, 510. [CrossRef]

366. Kumar, M.; Ando, Y. Chemical vapor deposition of carbon nanotubes: A review on growth mechanism and mass production. $J$. Nanosci. Nanotechnol. 2010, 10, 3739-3758. [CrossRef]

367. Meyyappan, M. A review of plasma enhanced chemical vapour deposition of carbon nanotubes. J. Phys. D 2009, $42,213001$. [CrossRef]

368. Hamzah, N.; Mohd Yasin, M.F.; Modh Yusop, M.Z.; Saatac, A.; Mohd Subhae, N.A. Rapid production of carbon nanotubes: A review on advancement in growth control and morphology manipulations of flame synthesis. J. Mater. Chem. A 2017, 5, 25144-25170. [CrossRef]

369. Merchan-Merchan, W.; Saveliev, A.V.; Kennedy, L.; Jimenez, W.C. Combustion synthesis of carbon nanotubes and related nanostructures. Prog. Energy Combust. Sci. 2010, 36, 696-727. [CrossRef]

370. Sharma, R.; Sharma, A.K.; Sharma, V. Synthesis of carbon nanotubes by arc-discharge and chemical vapor deposition method with analysis of its morphology, dispersion and functionalization characteristics. Cogent Eng. 2015, 2, 1094017. [CrossRef] 
371. Li, H.; Yuan, G.; Shan, B.; Zhang, X.; Ma, H.; Tian, Y.; Lu, H.; Liu, J. Chemical Vapor Deposition of Vertically Aligned Carbon Nanotube Arrays: Critical Effects of Oxide Buffer Layers. Nanoscale Res. Lett. 2019, 14, 106. [CrossRef]

372. Saurakhiya, N.; Zhu, Y.W.; Cheong, F.C.; Ong, C.K.; Wee, A.T.S.; Lin, J.Y.; Sow, C.H. Pulsed laser deposition-assisted patterning of aligned carbon nanotubes modified by focused laser beam for efficient field emission. Carbon 2005, 43, 2128-2133. [CrossRef]

373. Ismail, A.F.; Goh, P.S.; Tee, J.C.; Sanip, S.M. A review of purification techniques for carbon nanotubes. Nano 2008, 3, 127-143. [CrossRef]

374. Hou, P.-X.; Liu, C.; Cheng, H.-M. Purification of carbon nanotubes. Carbon 2008, 46, 2003-2025. [CrossRef]

375. Aqel, A.; Abou El-Nour, K.M.M.; Ammar, R.A.A. Carbon nanotubes, science and technology part (I) structure, synthesis and characterisation. Arab. J. Chem. 2012, 5, 1-23. [CrossRef]

376. Arnold, M.S.; Green, A.A.; Hulvat, J.F.; Stupp, S.I.; Hersam, M.C. Sorting Carbon Nanotubes by Electronic Structure Using Density Differentiation. Nat. Nanotechnol. 2006, 1, 60-65. [CrossRef] [PubMed]

377. Hu, H.; Yu, A.P.; Kim, E.; Zhao, B.; Itkis, M.E.; Bekyarova, E.; Haddon, R.C. Influence of the zeta potential on the dispersability and purification of single-walled carbon nanotubes. J. Phys. Chem. 2005, 109, 11520-11524. [CrossRef] [PubMed]

378. Chen, J.; Hamon, M.A.; Hu, H.; Chen, Y.; Rao, A.M.; Eklund, P.C.; Haddon, R.C. Solution Properties of Single-Walled Carbon Nanotubes. Science 1998, 282, 95-98. [CrossRef]

379. Lu, F.; Gu, L.; Meziani, M.J.; Wang, X.; Luo, P.G.; Veca, L.M.; Cao, L.; Sun, Y.-P. Advances in Bioapplications of Carbon Nanotubes. Adv. Mater. 2009, 21, 139-152.

380. Esumi, K.; Ishigami, M.; Nakajima, A.; Sawada, K.; Honda, H. Chemical treatment of carbon nanotubes. Carbon 1996, 34, $279-281$. [CrossRef]

381. Saleh, T.A. The influence of treatment temperature on the acidity of $\mathrm{MWCNT}$ oxidized by $\mathrm{HNO}_{3}$ or a mixture of $\mathrm{HNO}_{3} / \mathrm{H}_{2} \mathrm{SO}_{4}$. Appl. Surf. Sci. 2011, 257, 7746-7751. [CrossRef]

382. Yu, R.; Chen, L.; Liu, Q.; Lin, J.; Tan, K.-L.; Ng, S.C.; Chan, H.S.O.; Xu, G.-Q.; Hor, T.S.A. Platinum Deposition on Carbon Nanotubes via Chemical Modification. Chem. Mater. 1998, 10, 718-722. [CrossRef]

383. Sham, M.-L.; Kim, J.-K. Surface functionalities of multi-wall carbon nanotubes after UV/Ozone and TETA treatments. Carbon 2006, 44, 768-777. [CrossRef]

384. Wang, S.C.; Chang, K.S.; Yuan, C.J. Enhancement of electrochemical properties of screen-printed carbon electrodes by oxygen plasma treatment. Electrochim. Acta 2009, 54, 4937-4943. [CrossRef]

385. Gao, C.; Guo, Z.; Liu, J.-H.; Huang, X.-J. The new age of carbon nanotubes: An updated review of functionalized carbon nanotubes in electrochemical sensors. Nanoscale 2012, 4, 1948-1963. [CrossRef] [PubMed]

386. Hamon, M.A.; Hui, H.; Bhowmik, P. Ester-functionalized soluble single-walled carbon nanotubes. Appl. Phys. A 2002, 74, 333-338. [CrossRef]

387. Liu, J.; Rinzler, A.G.; Dai, H.; Hafner, J.H.; Bradley, R.K.; Boul, P.J.; Lu, A.; Iverson, T.; Shelimov, K.; Huffman, C.B.; et al. Fullerene Pipes. Science 1998, 280, 1253-1256. [CrossRef]

388. Ma, P.-C.; Kim, J.K.; Tang, B.Z. Functionalization of carbon nanotubes using a silane coupling agent. Carbon 2006, 44, 3232-3238. [CrossRef]

389. Sano, M.; Kamino, A.; Okamura, J.; Shinkai, S. Self-Organization of PEO-graft-Single-Walled Carbon Nanotubes in Solutions and Langmuir-Blodgett Films. Langmuir 2001, 17, 5125-5128. [CrossRef]

390. Coleman, J.N.; Khan, U.; Gun'ko, Y.K. Mechanical Reinforcement of Polymers Using Carbon Nanotubes. Adv. Mater. 2006, 18, 689-706. [CrossRef]

391. Balasubramanian, K.; Burghard, M. Chemically Functionalized Carbon Nanotubes. Small 2005, 1, 180-192. [CrossRef] [PubMed]

392. Touhara, H.; Inahara, J.; Mizuno, T.; Yokoyama, Y.; Okanao, S.; Yanagiuch, K.; Mukopadhyay, I.; Kawasaki, S.; Okino, F.; Shirai, H.; et al. Fluorination of cup-stacked carbon nanotubes, structure and properties. Fluor. Chem. 2002, 114, 181-188. [CrossRef]

393. Stevens, J.L.; Huang, A.Y.; Peng, H.; Chiang, I.W.; Khabashesku, V.N.; Margrave, J.L. Sidewall amino-functionalization of SWNTs through fluorination and subsequent reactions with terminal diamines. Nano Lett. 2003, 3, 331-336. [CrossRef]

394. Munirasu, S.; Albuerne, J.; Boschetti de Fierro, A.; Abetz, V. Functionalization of Carbon Materials using the Diels-Alder Reaction. Macromol. Rapid Commun. 2010, 31, 574-579. [CrossRef]

395. Hu, H.; Zhao, B.; Hamon, M.A.; Kamaras, K.; Itkis, M.E.; Haddon, R.C. Sidewall functionalization of single-walled carbon nanotubes by addition of dichlorocarbene. J. Am. Chem. Soc. 2003, 125, 14893-14900. [CrossRef]

396. Unger, E.; Graham, A.; Krepul, F.; Liebau, M.; Hoelein, W. Electrochemical functionalization of multi-walled carbon nanotubes for solvation and purification. Curr. Appl. Phys. 2002, 2, 107-111. [CrossRef]

397. Tagmatarchis, N.; Prato, M.J. Functionalization of carbon nanotubes via 1,3-dipolar cycloadditions. J. Mater. Chem. 2004, 14, 437-439. [CrossRef]

398. Kim, K.S.; Bae, D.J.; Kim, J.R.; Park, K.A.; Lim, S.C.; Kim, J.J.; Choi, W.B.; Park, C.J.; Lee, Y.H. Modification of Electronic Structures of a Carbon Nanotube by Hydrogen Functionalization. Adv. Mater. 2002, 14, 1818-1821. [CrossRef]

399. Moniruzzaman, M.; Winey, K.I. Polymer Nanocomposites Containing Carbon Nanotubes. Macromolecules 2006, $36,5194-5205$. [CrossRef]

400. Zhou, Y.; Fang, Y.; Ramasay, R.P. Non-Covalent Functionalization of Carbon Nanotubes for Electrochemical Biosensor Development. Sensors 2019, 19, 392. [CrossRef] [PubMed] 
401. Assali, M.; Pernia Leal, M.; Fernandez, I.; Baati, R.; Mioskowski, C.; Khiar, N. Non-covalent functionalization of carbon nanotubes with glycolipids: Glyconanomaterials with specific lectin-affinity. Soft Matt. 2009, 5, 948-950. [CrossRef]

402. Mallakpour, S.; Soltanian, S. Surface functionalization of carbon nanotubes: Fabrication and applications. RSC Adv. 2016, 6, 109916-109935. [CrossRef]

403. Liu, P. Modifications of carbon nanotubes with polymers. Eur. Polym. J. 2005, 41, 2693-2703. [CrossRef]

404. Li, X.; Wong, S.Y.; Tjiu, W.C.; Lyons, B.P.; Oh, S.A.; He, C.B. Non-covalent functionalization of multi walled carbon nanotubes and their application for conductive composites. Carbon 2008, 46, 829-831. [CrossRef]

405. Hsueh, C.C.; Brajter-Toth, A. Electrochemical Preparation and Analytical Applications of Ultrathin Overoxidized Polypyrrole Films. Anal. Chem. 1994, 66, 2458-2464. [CrossRef]

406. Yogeswaran, U.; Chen, M. Multi-walled carbon nanotubes with poly(methylene blue) composite film for the enhancement and separation of electroanalytical responses of catecholamine and ascorbic acid. Sens. Actuators B 2008, 130, 739-749. [CrossRef]

407. Yogeswaran, U.; Chen, M. Separation and concentration effect of f-MWCNTs on electrocatalytic responses of ascorbic acid, dopamine and uric acid at f-MWCNTs incorporated with poly (neutral red) composite films. Electrochim. Acta 2007, 52, 5985-5996. [CrossRef]

408. Wang, H.S.; Li, T.H.; Jia, W.L.; Xu, H.Y. Highly selective and sensitive determination of dopamine using a Nafion/carbon nanotubes coated poly(3-methylthiophene) modified electrode. Biosens. Bioelectron. 2006, 22, 664-669. [CrossRef]

409. Liu, A.; Honma, I.; Zhou, H. Simultaneous voltammetric detection of dopamine and uric acid at their physiological level in the presence of ascorbic acid using poly(acrylic acid)-multiwalled carbon-nanotube composite-covered glassy-carbon electrode. Biosens. Bioelectron. 2007, 23, 74-80. [CrossRef]

410. Wei, B.G.; Zhang, L.Y.; Chen, G. A multi-walled carbon nanotube/poly(urea-formaldehyde) composite prepared by in situpolycondensation for enhanced electrochemical sensing. New J. Chem. 2010, 34, 453-457. [CrossRef]

411. Fujigaya, T.; Nakashima, N. Non-covalent polymer wrapping of carbon nanotubes and the role of wrapped polymers as functional dispersants. Sci. Technol. Adv. Mater. 2015, 16, 024802. [CrossRef]

412. Perez, E.M.; Martin, N. $\pi-\pi$ interactions in carbon nanostructures. Chem. Soc. Rev. 2015, 44, 6425-6433. [CrossRef] [PubMed]

413. Shaoo, N.G.; Rana, S.; Cho, J.W.; Li, L.; Chan, S.H. Polymer nanocomposites based on functionalized carbon nanotubes. Prog. Polym. Sci. 2010, 35, 837-867. [CrossRef]

414. Chen, Z.; Zhang, A.; Wang, X.; Zhu, J.; Fan, Y.; Yu, H.; Yang, Z. The Advances of Carbon Nanotubes in Cancer Diagnostics and Therapeutics. J. Nanomater. 2017, 2017, 3418932. [CrossRef]

415. Jeon, I.-Y.; Chang, D.W.; Kumar, N.A.; Baek, J.-B. Chapter 5: Functionalization of Carbon Nanotubes. In Carbon Nanotubes-Polymer Nanocomposites; IntechOpen: London, UK, 2011; ISBN 978-953-51-4458-8. [CrossRef]

416. Cao, Y.; Cong, S.; Cao, X.; Wu, F.; Liu, Q.; Amer, M.R.; Zhou, C. Review of Electronics Based on Single-Walled Carbon Nanotubes. In Single-Walled Carbon Nanotubes: Preparation, Properties and Applications; Li, Y., Maruyama, S., Eds.; Springer International Publishing: Cham, Switzerland, 2019; pp. 189-224. ISBN 978-3-030-12700-8.

417. Park, S.; Vosguerichian, M.; Bao, Z. A Review of Fabrication and Applications of Carbon Nanotube Film-Based Flexible Electronics. Nanoscale 2013, 5, 1727-1752. [CrossRef] [PubMed]

418. Yan, Y.; Li, C.; Liu, C.; Mutlu, Z.; Dong, B.; Liu, J.; Ozkan, C.S.; Ozkan, M. Bundled and dispersed carbon nanotube assemblies on graphite superstructures as free-standing lithium-ion battery anodes. Carbon 2019, 142, 238-244. [CrossRef]

419. Song, B.; Xu, P.; Zeng, G.; Gong, J.; Zhang, P.; Feng, H.; Liu, Y.; Ren, X. Carbon nanotube-based environmental technologies: The adopted properties, primary mechanisms, and challenges. Rev. Environ. Sci. Biotechnol. 2018, 17, 571-590. [CrossRef]

420. Slattery, A.D.; Shearer, C.J.; Shapter, J.G.; Blanch, A.J.; Quinton, J.S.; Gibson, C.T. Improved Application of Carbon Nanotube Atomic Force Microscopy Probes Using PeakForce Tapping Mode. Nanomaterials 2018, 8, 807. [CrossRef]

421. Vashist, A.; Kaushik, A.; Vashist, A.; Sagar, V.; Ghosal, A.; Gupta, Y.K.; Ahmad, S.; Nair, M. Advances in Carbon NanotubesHydrogel Hybrids in Nanomedicine for Therapeutics. Adv. Healthc. Mater. 2018, 7, 1701213. [CrossRef]

422. Wang, J. Carbon-Nanotube Based Electrochemical Biosensors: A Review. Eelectroanalysis 2005, 17, 7-14. [CrossRef]

423. Meyyappan, M. Carbon Nanotube-Based Chemical Sensors. Small 2016, 12, 2116-2129. [CrossRef] [PubMed]

424. Kauffman, D.R.; Star, A. Carbon Nanotube Gas and Vapor Sensors. Angew. Chem. Int. Ed. 2008, 47, 6550-6570. [CrossRef] [PubMed]

425. Kong, J.; Franklin, N.R.; Zhou, C.; Chaplin, M.G.; Peng, S.; Cho, K.; Dai, H. Nanotube Molecular Wires as Chemical Sensors. Science 2000, 287, 622-625. [CrossRef] [PubMed]

426. Collins, P.G.; Bradley, K.; Ishigami, M.; Zettl, A. Extreme Oxygen Sensitivity of Electronic Properties of Carbon Nanotubes. Science 2000, 287, 1801-1804. [CrossRef]

427. Kang, D.; Park, N.; Ko, J.; Bae, E.; Park, W. Oxygen-Induced p-type Doping of a Long Individual Single-Walled Carbon Nanotube. Nanotechnology 2005, 16, 1048-1052. [CrossRef]

428. Schroeder, V.; Savagatrup, S.; He, M.; Lin, S.; Swager, T.M. Carbon Nanotube Chemical Sensors. Chem. Rev. 2019, 119, 599-663. [CrossRef]

429. Heller, I.; Janssen, A.M.; Mannik, J.; Minot, E.D.; Lemay, S.; Dekker, C. Identifying the Mechanism of Biosensing with Carbon Nanotube Transistors. Nano Lett. 2008, 8, 591-595. [CrossRef]

430. Yim, W.-L.; Gong, X.G.; Liu, Z.-F. Chemisorption of NO2 on Carbon Nanotubes. J. Phys. Chem. B 2003, 107, 9363-9369. [CrossRef] 
431. Rigoni, F.; Tognolini, S.; Borghetti, P.; Drera, G.; Pagliara, S. Enhancing the Sensitivity of Chemiresistor Gas Sensors Based on Pristine Carbon Nanotubes to Detect Low-ppb Ammonia Concentrations in the Environment. Analyst 2013, 138, 7392-7399. [CrossRef] [PubMed]

432. Salehi-Khojin, A.; Khalili-Araghi, F.; Kuroda, M.A.; Lin, K.Y.; Leburton, J.-P.; Masel, R.I. On the Sensing Mechanism in Carbon Nanotube Chemiresistors. ACS Nano 2011, 5, 153-158. [CrossRef]

433. Mirica, K.A.; Weis, J.G.; Schnorr, J.M.; Esser, B.; Swager, T.M. Mechanical Drawing of Gas Sensors on Paper. Angeww. Chem. Int. Ed. 2012, 51, 10740-10745. [CrossRef] [PubMed]

434. Yoon, B.; Liu, S.F.; Swager, T.M. Surface-Anchored Poly(4-Vinylpyridine)-Single-Walled Carbon Nanotube-Metal Composites for Gas Detection. Chem. Mater. 2016, 28, 5916-5924. [CrossRef]

435. Li, J.; Lu, Y.; Ye, Q.; Cinke, M.; Han, J.; Meyyappan, M. Carbon Nanotube Sensors for Gas and Organic Vapor Detection. Nano Lett. 2003, 3, 929-933. [CrossRef]

436. Cantalini, C.; Valentini, L.; Lozzi, L.; Armentano, I.; Kenny, J.M.; Santucci, S.N. $\mathrm{NO}_{2}$ Gas Sensitivity of Carbon Nanotubes Obtained by Plasma Enhanced Chemical Vapor Deposition. Sens. Actuators B 2003, 93, 333-337. [CrossRef]

437. Mubeen, S.; Lai, M.; Zhang, T.; Lim, J.H.; Mulchandani, A.; Deshusses, M.A.; Myung, N.V. Hybrid Tin Oxide-SWNT Nanostructures Based Gas Sensor. Electrochim. Acta 2013, 92, 484-490. [CrossRef]

438. Hoa, N.D.; Van Quy, N.; Kim, D. Nanowire Structured SnOx-SWNT Composites: High Performance Sensor for NOx Detection. Sens. Actuators B 2009, 142, 253-259. [CrossRef]

439. Ghaddab, B.; Sanchez, J.B.; Mavon, C.; Paillet, M.; Parret, R.; Zahab, A.A.; Bantignies, J.-L.; Flaud, V.; Beche, E.; Berger, F. Detection of $\mathrm{O} 3$ and NH3 Using Hybrid Tin Dioxide/Carbon Nanotubes Sensors: Influence of Materials and Processing on Sensor's Sensitivity. Sens. Actuators B 2012, 170, 67-74. [CrossRef]

440. Linag, X.; Chen, Z.; Wu, H.; Guo, L.; He, C.; Wang, B.; Wu, Y. Enhanced $\mathrm{NH}_{3}$-Sensing Behavior of 2,9,16,23-Tetrakis(2,2,3,3Tetrafluoropropoxy) Metal(II) Phthalocyanine/Multi-Walled Carbon Nanotube Hybrids: An Investigation of the Effects of Central Metals. Carbon 2014, 80, 268-278. [CrossRef]

441. Penza, M.; Rossi, R.; Alvisi, M.; Serra, E. Metal-modified and vertically aligned carbon nanotube sensors array for landfill gas monitoring applications. Nanotechnology 2010, 21, 105501. [CrossRef] [PubMed]

442. Kumar, M.K.; Ramaprabhu, S. Nanostructured Pt Functionlized Multiwalled Carbon Nanotube Based Hydrogen Sensor. J. Phys. Chem. B 2006, 110, 11291-11298. [CrossRef]

443. Penner, R.M. A Nose for Hydrogen Gas: Fast, Sensitive H2 Sensors Using Electrodeposited Nanomaterials. Acc. Chem. Res. 2017, 50, 1902-1910. [CrossRef] [PubMed]

444. Lu, Y.; Li, J.; Han, J.; Ng, H.T.; Binder, C.; Partridge, C.; Meyyappan, M. Room Temperature Methane Detection Using Palladium Loaded Single-Walled Carbon Nanotube Sensors. Chem. Phys. Lett. 2004, 391, 344-348. [CrossRef]

445. Humayun, M.T.; Divan, R.; Liu, Y.; Gundel, L.; Solomon, P.A.; Paprotny, I. Novel Chemoresistive CH4 Sensor with 10 ppm Sensitivity Based on Multiwalled Carbon Nanotubes Functionalized with SnO2 Nanocrystals. J. Vac. Sci. Technol. A 2016, 34, 01A131. [CrossRef]

446. Humayun, M.T.; Divan, R.; Stan, L.; Gupta, A.; Rosenmann, D.; Gundel, L.; Solomon, P.A.; Paprotny, I. ZnO Functionalization of Multiwalled Carbon Nanotubes for Methane Sensing at Single Parts per Million Concentration Levels. J. Vac. Sci. Technol. B 2015, 33, 06FF01. [CrossRef]

447. Savagatrup, S.; Schroeder, V.; He, X.; Lin, S.; He, M.; Yassine, O.; Salama, K.M.; Zhang, X.-X.; Swager, T.M. Bio-Inspired Carbon Monoxide Sensors with Voltage-Activated Sensitivity. Angew. Chem. Int. Ed. 2017, 56, 14066-14070. [CrossRef]

448. Peng, S.; Cho, K.J. Ab Initio Study of Doped Carbon Nanotube Sensors. Nano Lett. 2003, 3, 513-517. [CrossRef]

449. Zhao, W.; Fam, D.W.H.; Yin, Z.; Sun, T.; Tan, H.T.; Liu, W.; Tok, A.I.Y.; Boey, Y.C.F.; Zhang, H.; Hng, H.H. A Carbon Monoxide Gas Sensor Using Oxygen Plasma Modified Carbon Nanotubes. Nanotechnology 2012, 23, 425502/1-425502/6.

450. Liu, S.F.; Lin, S.; Swager, T.M. An Organocobalt-Carbon Nanotube Chemiresistive Carbon Monoxide Detector. ACS Sens. 2016, 1, 354-357. [CrossRef]

451. Kauffman, D.R.; Sorescu, D.C.; Schofield, D.P.; Allen, B.L.; Jordan, K.D.; Star, A. Understanding the Sensor Response of Metal-Decorated Carbon Nanotubes. Nano Lett. 2010, 10, 958-963. [CrossRef]

452. Yoosefian, M.; Barzgari, Z.; Yosefian, J. Ab Initio Study of Pd-Decorated Single-Walled Carbon Nanotube with C-Vacancy as CO Sensor. Struct. Chem. 2014, 25, 9-19. [CrossRef]

453. Zhang, Y.; Cui, S.; Chang, J.; Ocola, L.E.; Chen, J. Highly Sensitive Room Temperature Carbon Monoxide Detection Using SnO 2 Nanoparticle-Decorated Semiconducting Single-Walled Carbon Nanotubes. Nanotechnology 2013, 24, 025503. [CrossRef]

454. Mubeen, S.; Zhang, T.; Chartuprayoon, N.; Rheem, Y.; Mulchandani, A.; Myung, N.V.; Deshusses, M.A. Sensitive Detection of H2S Using Gold Nanoparticle Decorated Single-Walled Carbon Nanotubes. Anal. Chem. 2010, 82, 250-257. [CrossRef]

455. Yu, S.Y.S.; Yi, W.Y.W. Single-Walled Carbon Nanotubes as a Chemical Sensor for $\mathrm{SO}_{2}$ Detection. IEEE Trans. Nanotechnol. 2007, 6, 545-548. [CrossRef]

456. Rushi, A.D.; Datta, K.P.; Ghosh, P.S.; Mulchandani, A. Selective Discrimination among Benzene, Toluene, and Xylene: Probing Metalloporphyrin-Functionalized Single-Walled Carbon Nanotube-Based Field Effect Transistors. J. Phys. Chem. C 2014, 118, 24034-24041. [CrossRef]

457. Hafaiedh, I.; El Evch, W.; Clement, P.; Llobet, E.; Abdelghani, A. Multi-walled carbon nanotubes for volatile organic compound detection. Sens. Actuators B 2013, 182, 344-350. [CrossRef] 
458. Sarkar, T.; Srinives, S.; Sarkar, S.; Haddon, R.C.; Mulchendani, A. Single-Walled Carbon Nanotube-Poly(porphyrin) Hybrid for Volatile Organic Compounds Detection. J. Phys. Chem. C 2014, 118, 1602-1610. [CrossRef]

459. Tasaltin, C.; Basarir, F. Preparation of flexible VOC sensor based on carbon nanotubes and gold nanoparticles. Sens. Actuators $B$ 2014, 194, 173-179. [CrossRef]

460. Liao, Y.; Zhang, C.; Zhang, Y.; Strong, V.; Tang, J.; Li, X.G.; Kalantar-Zadeh, K.; Hoek, E.M.V.; Wang, K.L.; Kaner, R.B. Carbon Nanotube/Polyaniline Composite Nanofibers: Facile Synthesis and Chemosensors. Nano Lett. 2011, 11, 954-959. [CrossRef]

461. Gou, P.; Kraut, N.D.; Feigel, I.M.; Bai, H.; Morgan, G.J.; Chen, Y.; Tang, Y.; Bocan, K.; Stanchel, J.; Berger, L.; et al. Carbon Nanotube Chemiresistor for Wireless PH Sensing. Sci Rep. 2015, 4, 4468. [CrossRef] [PubMed]

462. Li, P.; Martin, C.; Yeung, K.K.; Xue, W. Dielectrophoresis Aligned Single-Walled Carbon Nanotubes as PH Sensors. Biosensors 2011, 1, 23-25. [CrossRef] [PubMed]

463. Muenzer, A.M.; Melzer, K.; Heimgreiter, M.; Scarpa, G. Random CNT Network and Regioregular Poly(3-hexylthiophen) FETs for PH Sensing Applications: A Comparison. Biochim. Biophys. Acta 2013, 1830, 4353-4358. [CrossRef] [PubMed]

464. Takeda, S.; Nakanura, M.; Ishii, A.; Subagyo, A.; Hosoi, H.; Sueoka, K.; Mukasa, K. A PH Sensor Based on Electric Properties of Nanotubes on a Glass Substrate. Nanoscale Res. Lett. 2007, 2, 207-212. [CrossRef] [PubMed]

465. Leghrib, R.; Llobet, E.; Pavelko, R.; Vasiliev, A.A.; Felten, A.; Pireaux, J.J. Gas Sensing Properties of MWCNTs Decorated with Gold or Tin Oxide Nanoparticles. Procedia Chem. 2009, 1, 168-171. [CrossRef]

466. Schnorr, J.M.; Swager, T.M. Selective Detection of Ethylene Gas Using Carbon Nanotube-Based Devices: Utility in Determination of Fruit Ripeness. Angew. Chem. Int. Ed. 2012, 51, 5752-5756.

467. Jin, H.J.; Lee, S.H.; Kim, T.H.; Park, J.; Song, H.S.; Park, T.H.; Hong, S. Nanovesicle-Based Bioelectronic Nose Platform Mimicking Human Olfactory Signal Transduction. Biosens. Bioelectron. 2012, 35, 335-341. [CrossRef] [PubMed]

468. Kim, T.H.; Lee, S.H.; Lee, J.; Song, H.S.; Oh, E.H.; Park, T.H.; Hong, S. Single-Carbon-Atomic-Resolution Detection of Odorant Molecules Using a Human Olfactory Receptor-Based Bioelectronic Nose. Adv. Mater. 2009, 21, 91-94. [CrossRef]

469. Liu, S.F.; Petty, A.R.; Sazama, G.T.; Swager, T.M. Single-Walled Carbon Nanotube/Metalloporphyrin Composites for the Chemiresistive Detection of Amines and Meat Spoilage. Angew. Chem. Int. Ed. 2015, 54, 6554-6557. [CrossRef]

470. Mills, A. Oxygen Indicators and Intelligent Inks for Packaging. Chem. Soc. Rev. 2005, 34, 1003-1011. [CrossRef]

471. Cai, C.; Chen, J. Direct electron transfer of glucose oxidase promoted by carbon nanotubes. Anal. Biochem. $2004,332,75-83$.

472. Tang, H.; Chen, J.; Yao, S.; Nie, L.; Deng, G.; Kuang, Y. Amperometric Glucose Biosensor Based on Adsorption of Glucose Oxidase at Platinum Nanoparticle-Modified Carbon Nanotube Electrode. Anal. Biochem. 2004, 311, 89-97. [CrossRef]

473. Patolsky, F.; Weizmann, Y.; Willner, I. Long-range electrical contacting of redox enzymes by SWCNT connectors. Angew. Chem. Int. Ed. 2004, 43, 2113-2117. [CrossRef] [PubMed]

474. Pilan, L.; Raicopol, M. Highly Selective and Stable Glucose Biosensors Based on Polyaniline/Carbon Nanotubes Composites. UPB Sci. Bull. B 2014, 78, 155-166.

475. Soylemez, S.; Yoon, B.; Toppare, L.; Swager, T.M. Quaternized Polymer-Single-Walled Carbon Nanotube Scaffolds for a Chemiresistive Glucose Sensor. ACS Sens. 2017, 2, 1123-1127. [CrossRef]

476. Li, G.; Liao, J.M.; Hu, G.Q.; Ma, N.Z.; Wu, P.J. Study of carbon nanotube modified biosensor for monitoring total cholesterol in blood. Biosens. Bioelectron. 2005, 20, 2140-2144. [CrossRef] [PubMed]

477. Wisitsoraat, A.; Karuwan, C.; Wong-ek, K.; Phokharatkul, D.; Sritongkham, P.; Tuantranont, A. High Sensitivity Electrochemical Cholesterol Sensor Utilizing a Vertically Aligned Carbon Nanotube Electrode with Electropolymerized Enzyme Immobilization. Sensors 2009, 9, 8658-8668. [CrossRef]

478. Wang, J.; Musameh, M. Electrochemical detection of trace insulin at carbon-nanotube-modified electrodes. Anal. Chim. Acta 2004, 511,33-36. [CrossRef]

479. Wang, J.; Tangkuaram, T.; Loyprasert, S.; Vazquez-Alvarez, T.; Veerasai, W.; Kanatharana, P.; Thavarungkul, P. Electrocatalytic detection of insulin at RuOx/carbon nanotube-modified carbon electrodes. Anal. Chim. Acta 2007, 581, 1-6. [CrossRef]

480. Iverson, N.M.; Hofferber, E.M.; Stapleton, J.A. Nitric Oxide Sensors for Biological Applications. Chemosensors 2018, 6, 8. [CrossRef]

481. Zhang, J.Q.; Boghossian, A.A.; Barone, P.W.; Rwei, A.; Kim, J.H.; Lin, D.H.; Heller, D.A.; Hilmer, A.J.; Nair, N.; Reuel, N.F.; et al. Single Molecule Detection of Nitric Oxide Enabled by d(AT)(15) DNA Adsorbed to NearInfrared Fluorescent Single-Walled Carbon Nanotubes. J. Am. Chem. Soc. 2011, 133, 567-581. [CrossRef]

482. Atolia, E.; Farias, E.; McNicholas, T.P.; Reuel, N.F.; Parry, N.M.A.; Wogan, G.N.; Strano, M.S. In vivo biosensing via tissuelocalizable near-infrared-fluorescent single-walledcarbon nanotubes. Nat. Nanotechnol. 2013, 8, 873-880.

483. Prasad, B.B.; Prasad, A.; Tiwari, M.P.; Madhuri, R. Multiwalled carbon nanotubes bearing "terminal monomeric unit" for the fabrication of epinephrine imprinted polymer-based electrochemical sensor. Biosens. Bioelectron. 2013, 45, 114-122. [CrossRef] [PubMed]

484. Caetano, F.R.; Felippe, L.B.; Zarbin, A.J.; Bergamini, M.F.; Marcolino-Junior, L.H. Gold nanoparticles supported on multi-walled carbon nanotubes produced by biphasic modified method and dopamine sensing application. Sens. Actuators B 2017, 243, 43-50. [CrossRef]

485. Bala, K.; Sharma, D.; Gupta, N. Carbon-Nanotube-Based Materials for Electrochemical Sensing of the Neurotransmitter Dopamine. ChemElectroChem 2019, 6, 274-288. [CrossRef] 
486. Jin, H.; Heller, D.A.; Kalbacova, M.; Kim, J.-H.; Zhang, J.; Boghossian, A.A.; Maheshri, N.; Strano, M.S. Detection of singlemolecule $\mathrm{H}_{2} \mathrm{O}_{2}$ signalling from epidermal growth factor receptor using fluorescent single-walled carbon nanotubes. Nat. Nanotechnol. 2010, 5, 302-309. [CrossRef] [PubMed]

487. Tang, X.; Bansarunti, S.; Nakayama, N.; Yenilmez, E.; Chang, Y.-L.; Wang, Q. Carbon Nanotube DNA Sensor and Sensing Mechanism. Nano Lett. 2006, 6, 1632-1636. [CrossRef] [PubMed]

488. Wang, J.; Liu, G.; Jan, M.R. Ultrasensitive Electrical Biosensing of Proteins and DNA: Carbon-Nanotube Derived Amplification of the Recognition and Transduction Events. J. Am. Chem. Soc. 2004, 126, 3010-3011. [CrossRef]

489. Balasubramanian, K.; Burghard, M. Biosensors Based on Carbon Nanotubes. Anal. Bioanal. Chem. 2006, 385, 452-468. [CrossRef]

490. Li, W.; Gao, Y.; Zhang, J.; Wang, X.; Yin, F.; Li, Z.; Zhang, M. Universal DNA detection realized by peptide based carbon nanotube biosensors. Nanoscale Adv. 2020, 2, 717-723. [CrossRef]

491. Li, J.; Lee, E.-C. Functionalized multi-wall carbon nanotubes as an efficient additive for electrochemical DNA sensor. Sens. Actuators B 2017, 239, 652-659. [CrossRef]

492. Morton, J.; Havens, N.; Mugweru, A.; Wanekaya, A. Detection of Trace Heavy Metal Ions Using Carbon Nanotube modified Electrodes. Electroanalysis 2009, 21, 1597-1603.

493. Zaporotskova, I.V.; Boroznina, N.P.; Parkhomenko, Y.N.; Kozhitovb, L.V. Carbon nanotubes: Sensor properties. A review. Mod. Electr. Mater. 2016, 2, 95-105. [CrossRef]

494. Wang, T.; Wei, Y. Carbon nanotubes heavy metal detection with stripping voltammetry A review paper. Electroanalysis 2017, 29, 2178-2189. [CrossRef]

495. Wong, A.; Silva, T.A.; Caetano, F.R.; Bergamini, M.F.; Marcolinio-Jnr, L.H.; Fatibello-Filho, O.; Janegitz, B.C. An Overview of Pesticide Monitoring at Environmental Samples Using Carbon Nanotubes-Based Electrochemical Sensors. C J. Carbon Res. 2017, 3, 8. [CrossRef]

496. Velusamy, V.; Arshak, K.; Korostynska, O.; Oliwa, K.; Adley, C. An Overview of Foodborne Pathogen Detection: In the Perspective of Biosensors. Biotechnol. Adv. 2010, 28, 232-254. [CrossRef]

497. Schnorr, J.M.; van der Zwaag, D.; Walish, J.J.; Weizman, Y.; Swager, T.M. Sensory Arrays of Covalently Functionalized SingleWalled Carbon Nanotubes for Explosive Detection. Adv. Funct. Mater. 2013, 23, 5285-5291. [CrossRef]

498. Wan, Y.; Deng, W.; Su, Y.; Zhu, X.; Peng, C.; Hu, H.; Peng, H.; Song, S.; Fan, C. Carbon nanotube-based ultrasensitive multiplexing electrochemical immunosensor for cancer biomarkers. Biosens. Bioelectron. 2011, 30, 93-99. [CrossRef]

499. Liu, Z.; Tabakman, S.; Sherlock, S.; Li, X.; Chen, Z.; Jiang, K.; Fan, S.; Dai, H. Multiplexed Five-Color Molecular Imaging of Cancer Cells and Tumor Tissues with Carbon Nanotube Raman Tags in the Near-Infrared. Nano Res. 2010, 3, 222-233. [CrossRef]

500. Lee, C.; Wei, X.; Kysar, J.W.; Hone, J. Measurement of the Elastic Properties and Intrinsic Strength of Monolayer Graphene. Science 2008, 321, 385-388. [CrossRef]

501. Bolotin, K.I.; Sikes, K.J.; Jiang, Z.; Klima, M.; Fudenberg, G.; Hone, J.; Kim, P.; Stormer, H.L. Ultrahigh electron mobility in suspended graphene. Solid State Commun. 2008, 146, 351-355. [CrossRef]

502. Novoselov, K.S.; Morozov, S.V.; Mohinddin, T.M.G.; Ponomarenko, L.A.; Elias, D.C.; Yang, R.; Barbolina, I.I.; Blake, P.; Booth, T.J.; Jiang, D.; et al. Electronic properties of graphene. Phys. Stat. Sol. B 2007, 244, 4106-4111. [CrossRef]

503. Balandin, A.; Ghosh, S.; Bao, W.; Calizo, I.; Teweldebrhan, D.; Miao, F.; Lau, C.N. Superior Thermal Conductivity of Single-Layer Graphene. Nano Lett. 2008, 8, 902-907. [CrossRef] [PubMed]

504. Züttel, A.; Sudan, P.; Mauron, P.; Wenger, P. Model for the hydrogen adsorption on carbon nanostructures. Appl. Phys. A 2004, 78, 941-946. [CrossRef]

505. Nair, R.R.; Blake, P.; Grigorenko, A.N.; Novoselov, K.S.; Booth, T.J.; Stauber, T.; Peres, N.M.R.; Geim, A.K. Fine Structure Constant Defines Visual Transparency of Graphene. Science 2008, 320, 1308. [CrossRef]

506. Huang, H.; Chen, S.; Wee, A.T.S.; Chen, W. Chapter 1-Epitaxial growth of graphene on silicon carbide (SiC). In GrapheneProperties, Preparation, Characterization and Devices; Woodhead Publishing Ltd.: Cambridge, UK, 2014; pp. 3-26. ISBN 978-0-85709508-4.

507. Shtepliuk, I.; Khranovskyy, V.; Yakimova, R. Combining graphene with silicon carbide: Synthesis and properties-A review. Semicond. Sci. Technol. 2016, 31, 113004. [CrossRef]

508. Badami, D.V. X-Ray studies of graphite formed by decomposing silicon carbide. Carbon 1965, 3, 53-57. [CrossRef]

509. Van Bommel, A.J.; Crombeen, J.E.; Van Tooren, A. LEED and Auger electron observations of the SiC(0001) surface. Surf. Sci. 1975, 48, 463-472. [CrossRef]

510. Emtsev, K.V.; Bostwick, A.; Horn, K.; Jobst, J.; Kellogg, G.L.; Ley, L.; McChesney, J.L.; Ohta, T.; Reshanov, S.A.; Röhrl, J.; et al. Towards wafer-size graphene layers by atmospheric pressure graphitization of silicon carbide. Nat. Mater. 2009,8 , $203-207$. [CrossRef]

511. Tromp, R.M.; Hannon, J.B. Thermodynamics and Kinetics of Graphene Growth on SiC(0001). Phys. Rev. Lett. 2013, 102, 106104. [CrossRef]

512. Juang, Z.-Y.; Wu, C.-Y.; Lo, C.-W.; Chen, W.-Y.; Huang, C.-F.; Hwang, J.-C.; Chen, F.-R.; Leou, K.-C.; Tsai, C.-H. Synthesis of graphene on silicon carbide substrates at low temperature. Carbon 2009, 47, 2026-2031. [CrossRef]

513. Li, C.; Li, D.; Yang, J.; Zeng, X.; Yuan, W. Preparation of single- and few-layer graphene sheets using co deposition on SiC substrate. J. Nanomater. 2011, 2011, 1-7. [CrossRef] 
514. Wang, J.-B.; Ren, Z.; Hou, Y.; Yan, X.-L.; Liu, P.-Z.; Hua, Z.; Zhang, H.X.; Guo, J.J. A review of graphene synthesis at low temperatures by CVD methods. New Carbon Mater. 2020, 35, 193-208. [CrossRef]

515. Osikoya, A.O.; Parlak, O.; Murugan, N.A.; Dikio, E.D.; Moloto, H.; Uzun, L.; Turner, A.P.; Tiwari, A. Acetylene-sourced CVDsynthesised catalytically active graphene for electrochemical biosensing. Biosens. Bioelectron. 2017, 89, 496-504. [CrossRef] [PubMed]

516. Cushing, G.W.; Johánek, V.; Navin, J.K.; Harrison, I. Graphene Growth on Pt(111) by Ethylene Chemical Vapor Deposition at Surface Temperatures near 1000 K. J. Phys. Chem. C 2015, 119, 4759-4768. [CrossRef]

517. Gotterbarm, K.; Zhao, W.; Höfert, O.; Gleichweit, C.; Papp, C.; Steinrück, H.P. Growth and oxidation of graphene on Rh(111). Phys. Chem. Chem. Phys. 2013, 15, 19625-19631. [CrossRef]

518. Guermoune, A.; Chari, T.; Popescu, F.; Sabri, S.S.; Guillemette, J.; Skulason, H.S.; Szkopek, T.; Siaj, M. Chemical vapor deposition synthesis of graphene on copper with methanol, ethanol, and propanol precursors. Carbon 2011, 49, 4204-4210. [CrossRef]

519. Zhao, P.; Kumamoto, A.; Kim, S.; Chen, X.; Hou, B.; Chiashi, S.; Einarsson, E.; Ikuhara, Y.; Maruyama, S. Self-Limiting Chemical Vapor Deposition Growth of Monolayer Graphene from Ethanol. J. Phys. Chem. C 2013, 117, 10755-10763. [CrossRef]

520. Bautista, C.; Mendoza, D. Multilayer graphene synthesized by CVD using liquid hexane as the carbon precursor. arXiv 2011, arXiv:11091318.

521. Jang, J.; Son, M.; Chung, S.; Kim, K.; Cho, C.; Lee, B.H.; Ham, M.-H. Low-temperature-grown continuous graphene films from benzene by chemical vapor deposition at ambient pressure. Sci. Rep. 2015, 5, 17955. [CrossRef]

522. Zhengzong, S.; Zheng, Y.; Yao, J.; Beitler, E.; Zhu, Y.; Tour, J.M. Growth of graphene from solid carbon sources. Nature 2010, 468, 549-552. [CrossRef]

523. Ji, H.; Hao, Y.; Ren, Y.; Charlton, M.; Lee, W.H.; Wu, Q.; Li, H.; Zhu, Y.; Wu, Y.; Piner, R.; et al. Graphene Growth Using a Solid Carbon Feedstock and Hydrogen. ACS Nano 2011, 5, 7656-7661. [CrossRef]

524. Zhang, Y.; Zhang, L.; Zhou, C. Review of Chemical Vapor Deposition of Graphene and Related Applications. Acc. Chem. Res. 2013, 46, 2329-2339. [CrossRef]

525. Liu, M. Controllable Synthesis of Graphene on Rh. In Controlled Synthesis and Scanning Tunneling Microscopy Study of Graphene and Graphene-Based Heterostructures; Springer: Singapore, 2018; pp. 19-35. ISBN 978-981-10-5181-4.

526. Wang, S.M.; Pei, Y.H.; Wang, X.; Wang, H.; Meng, Q.N.; Tian, H.W.; Zheng, X.L.; Zheng, W.T.; Liu, Y.C. Synthesis of graphene on a polycrystalline Co film by radio-frequency plasma-enhanced chemical vapour deposition. J. Phys. Appl. Phys. 2010, $43,455402$. [CrossRef]

527. Dai, B.; Fu, L.; Zou, Z.; Wang, M.; Xu, H.; Wang, S.; Liu, Z. Rational design of a binary metal alloy for chemical vapour deposition growth of uniform single-layer graphene. Nat. Commun. 2011, 2, 522. [CrossRef]

528. Choi, H.; Lim, Y.; Park, M.; Lee, S.; Kang, Y.; Kim, M.S.; Kim, J.; Jeon, M. Precise control of chemical vapor deposition graphene layer thickness using NixCu1-x alloys. J. Mater. Chem. C 2015, 3, 1463-1467. [CrossRef]

529. Wu, T.; Zhang, X.; Yuan, Q.; Xue, J.; Lu, G.; Liu, Z.; Wang, H.; Wang, H.; Ding, F.; Yu, Q.; et al. Fast growth of inch-sized single-crystalline graphene from a controlled single nucleus on $\mathrm{Cu}-\mathrm{Ni}$ alloys. Nat. Mater. 2015, 15, 43. [CrossRef] [PubMed]

530. Yang, X.; Zhang, G.; Prakash, J.; Chen, Z.; Gauthier, M.; Sun, S. Chemical vapour deposition of graphene: Layer control, the transfer process, characterisation, and related applications. Int. Rev. Phys. Chem. 2019, 38, 149-199. [CrossRef]

531. Li, M.; Liu, D.; Wei, D.; Song, X.; Wei, D.; Wee, A.T.S. Controllable Synthesis of Graphene by Plasma-Enhanced Chemical Vapor Deposition and Its Related Applications. Adv. Sci. 2016, 3, 1600003. [CrossRef] [PubMed]

532. Woehrl, N.; Ochedowski, O.; Gottlieb, S.; Shibasaki, K.; Schulz, S. Plasma-enhanced chemical vapor deposition of graphene on copper substrates. AIP Adv. 2014, 4, 047128. [CrossRef]

533. Robinson, J.; Weng, X.; Trumbull, K.; Cavalero, R.; Wetherington, M.; Frantz, E.; LaBella, M.; Hughes, Z.; Fanton, M.; Snydert, D. Nucleation of Epitaxial Graphene on SiC(0001). ACS Nano 2010, 4, 153-158. [CrossRef] [PubMed]

534. Fujita, J.; Hiyama, T.; Hirukawa, A.; Kondo, T.; Nakamura, J.; Ito, S.; Araki, R.; Ito, Y.; Takeguchi, M.; Pai, W.W. Near room temperature chemical vapor deposition of graphene with diluted methane and molten gallium catalyst. Sci. Rep. 2017, 7, 12371. [CrossRef]

535. Miseikis, V.; Convertino, D.; Mishra, N.; Gemmi, M.; Mashoff, T.; Heun, S.; Haghighian, N.; Bisio, F.; Canepa, M.; Piazza, V.; et al. Rapid CVD growth of millimetre-sized single crystal graphene using a cold-wall reactor. arXiv 2015, arXiv:1501.06356. [CrossRef]

536. Novoselov, K.S.; Geim, A.K.; Morozov, S.V.; Jiang, D.; Zhang, Y.; Dubonos, S.V.; Grigorieva, I.V.; Firsov, A.A. Electric Field Effect in Atomically Thin Carbon Films. Science 2004, 306, 666-669. [CrossRef]

537. Hernandez, Y.; Nicolosi, V.; Lotya, M.; Blighe, F.M.; Sun, Z.; De, S.; McGovern, I.T.; Holland, B.; Byrne, M.; Gun'Ko, Y.K.; et al. High-yield production of graphene by liquid-phase exfoliation of graphite. Nat. Nanotechnol. 2008, 3, 563-568. [CrossRef]

538. Knieke, C.; Berger, A.; Voigt, M.; Taylor, R.N.K.; Röhrl, J.; Peukert, W. Scalable production of graphene sheets by mechanical delamination. Carbon 2010, 48, 3196-3204. [CrossRef]

539. Lv, Y.; Yu, L.; Jiang, C.; Chen, S.; Nie, Z. Synthesis of graphene nanosheet powder with layer number control via a soluble salt-assisted route. RSC Adv. 2014, 4, 13350-13354. [CrossRef]

540. Hummers, W.S.; Offeman, R.E. Preparation of Graphitic Oxide. J. Am. Chem. Soc. 1958, 80, 1339. [CrossRef]

541. Quezada-Renteria, J.A.; Ania, C.O.; Chazaro-Ruiz, L.F.; Rangel-Mendez, J.R. Influence of protons on reduction degree and defect formation in electrochemically reduced graphene oxide. Carbon 2019, 149, 722-732. [CrossRef] 
542. Saleem, H.; Haneef, M.; Abbasi, H.Y. Synthesis route of reduced graphene oxide via thermal reduction of chemically exfoliated graphene oxide. Mater. Chem. Phys. 2018, 204, 1-7. [CrossRef]

543. Guex, L.G.; Sacchi, B.; Peuvot, K.F.; Andersson, R.L.; Pourrahimi, A.M.; Strom, V.; Farris, S.; Olsson, R.T. Experimental review: Chemical reduction of graphene oxide (GO) to reduced graphene oxide (rGO) by aqueous chemistry. Nanoscale 2017, 9, 9562-9571. [CrossRef] [PubMed]

544. Mei, X.; Meng, X.; Wu, F. Hydrothermal method for the production of reduced graphene oxide. Phys. E Low-Dimens. Syst. Nanostruct. 2015, 68, 81-86. [CrossRef]

545. Park, S.; An, J.; Potts, J.R.; Velamakanni, A.; Murali, S.; Ruoff, R.S. Hydrazine-reduction of graphite- and graphene oxide. Carbon 2011, 49, 3019-3023. [CrossRef]

546. Shin, H.-J.; Kim, K.K.; Benayad, A.; Yoon, S.-M.; Park, H.K.; Jung, I.-S.; Jin, M.H.; Jeong, H.-K.; Kim, J.M.; Choi, J.-Y.; et al. Efficient Reduction of Graphite Oxide by Sodium Borohydride and Its Effect on Electrical Conductance. Adv. Funct. Mater. 2009, 19, 1987-1992. [CrossRef]

547. Moon, I.K.; Lee, J.; Lee, H. Highly qualified reduced graphene oxides: The best chemical reduction. Chem. Commun. 2011, 47, 9681-9683. [CrossRef]

548. Zhang, J.; Yang, H.; Shen, G.; Cheng, P.; Zhang, J.; Guo, S. Reduction of graphene oxide vial-ascorbic acid. Chem. Commun. 2010, 46, 1112-1114. [CrossRef]

549. De Silva, K.K.H.; Huang, H.H.; Joshi, R.K.; Yoshimura, M. Chemical reduction of graphene oxide using green reductants. Carbon 2017, 119, 190-199. [CrossRef]

550. Khanra, P.; Kuila, T.; Kim, N.H.; Bae, S.H.; Yu, D.-S.; Lee, J.H. Simultaneous bio-functionalization and reduction of graphene oxide by baker's yeast. Chem. Eng. J. 2012, 183, 526-533. [CrossRef]

551. Dave, S.H.; Gong, C.; Robertson, A.W.; Warner, J.H.; Grossman, J.C. Chemistry and Structure of Graphene Oxide via Direct Imaging. ACS Nano 2016, 10, 7515-7522. [CrossRef]

552. Gomez-Navarro, C.; Meyer, J.C.; Sundaram, R.S.; Chuvilin, A.; Kurash, S.; Burghard, M.; Kern, K.; Kaiser, U. Atomic Structure of Reduced Graphene Oxide. Nano Lett. 2010, 10, 1144-1148. [CrossRef]

553. Yavari, F.; Kritzinger, C.; Gaire, C.; Song, L.; Gullapalli, H.; Borca-Tasciuc, T. Tunable bandgap in graphene by the controlled adsorption of water molecules. Small 2010, 6, 2535-2538. [CrossRef] [PubMed]

554. Chung, C.; Kim, Y.-K.; Shin, D.; Ryoo, S.-R.; Hong, B.-H.; Min, D.-H. Biomedical applications of graphene and graphene oxide. Acc. Chem Res. 2013, 46, 2211-2224. [CrossRef]

555. Viswanathan, P.; Ramaraj, R. Chapter 2-Functionalized Graphene Nanocomposites for Electrochemical Sensors. In GrapheneBased Electrochemical Sensors for Biomolecules Micro and Nano Technologies; Elsevier: Amsterdam, The Netherlands, 2019; ISBN 978-0-12-815394-9.

556. Rodrigues-Perez, L.; Herranz, M.A.; Martin, N. The chemistry of pristine graphene. Chem. Commun. 2013, 49, $3721-3735$. [CrossRef]

557. Allen, M.J.; Tung, V.C.; Kaner, R.B. Honeycomb carbon: A review of graphene. Chem. Commun. 2010, 110, 132-145. [CrossRef]

558. Brodie, B.C. On the atomic weight of graphite. Philos. Trans. R Soc. Lond. 1859, 14, $249-259$.

559. Dreyer, D.R.; Park, S.; Bielawski, C.W.; Ruoff, R.S. The chemistry of graphene oxide. Chem. Soc. Rev. 2010, 39, 228-240. [CrossRef]

560. Eda, G.; Chowalla, M. Chemically Derived Graphene Oxide: Towards Large-Area Thin-Film Electronics and Optoelectronics. Adv. Mater. 2010, 22, 2392-2415. [CrossRef]

561. Hontoria-Lucas, C.; Lopez-Peinado, A.; Lopez-Gonzalez, J.; Rojas-Cervantes, M.; Martin-Aranda, R. Study of oxygen- containing groups in a series of graphite oxides: Physical and chemical characterization. Carbon 1995, 33, 1585-1592. [CrossRef]

562. Vacchi, I.A.; Raya, J.; Bianco, A.; Ménard-Moyon, C. Controlled derivatization of hydroxyl groups of graphene oxide in mild conditions. 2D Mater. 2018, 5, 035037. [CrossRef]

563. Chen, D.; Feng, H.; Li, J. Graphene Oxide: Preparation, Functionalization, and Electrochemical Applications. Chem. Rev. 2012, 112, 6027-6053. [CrossRef]

564. Khan, A.A.; Aftab, A.P.; Khan, A.; Asiri, A.M.; Ashraf, G.; Alhogbia, B.G. Graphene Oxide Based Metallic Nanoparticles and their Some Biological and Environmental Application. Curr. Drug Metab. 2017, 18, 1020-1029. [CrossRef] [PubMed]

565. Liu, J.; Fu, S.; Yuan, B.; Li, Y.; Deng, Z. Toward a Universal “Adhesive Nanosheet" for the Assembly of Multiple Based on a Protein-Induced Reduction/of graphene oxide. J. Am. Chem. Soc. 2010, 132, 7279-7281. [CrossRef] [PubMed]

566. Toda, R.; Furue, R.; Hayami, S. Recent progress in applications of for gas sensing: A review. Anal. Chim. Acta 2015, 878, 43-53. [CrossRef]

567. Wang, Y.; Lu, J.; Tang, L.H.; Chang, H.X.; Li, J.H. Graphene Oxide Amplified Electrogenerated Chemiluminescence of Quantum Dots and Its Selective Sensing for Glutathione from Thiol-Containing Compounds. Anal. Chem. 2009, 81, 9710-9715. [CrossRef] [PubMed]

568. Vijesh, V.K.; Mathew, S.; Nampoori, V.P.N.; Sheenu, T. Carbon dots decorated graphene oxide nanosheets prepared by a novel technique with enhanced nonlinear optical properties. AIP Adv. 2019, 9, 015219.

569. Liu, X.W.; Yao, Z.J.; Wang, Y.F.; Wei, X.W. Graphene oxide sheet-prussian blue nanocomposites: Green synthesis and their extraordinary electrochemical properties. Coll. Surf. B 2010, 81, 508-512. [CrossRef]

570. Wang, H.L.; Casalongue, H.S.; Liang, Y.Y.; Dai, H.J. Ni(OH) 2 Nanoplates Grown on Graphene as Advanced Electrochemical Pseudocapacitor Materials. J. Am. Chem. Soc. 2010, 132, 7472-7477. [CrossRef] 
571. Karousis, N.; Economopoulos, S.P.; Sarantopoulou, E.; Tagmatarchis, N. Porphyrin counter anion in imidazolium-modified graphene-oxide. Carbon 2010, 48, 854-860. [CrossRef]

572. Liu, H.; Gao, J.; Xue, M.Q.; Zhu, N.; Zhang, M.N.; Cao, T.B. Processing of graphene for electrochemical application: Noncovalently functionalize graphene sheets with water-soluble electroactive methylene green. Langmuir 2009, 25, 12006. [CrossRef]

573. Stankovich, S.; Dikin, D.A.; Compton, O.C.; Dommett, G.H.B.; Ruoff, R.S.; Nguyen, S.T. Systematic Post-assembly Modification of Graphene Oxide Paper with Primary Alkylamines. Chem. Mater. 2010, 22, 4153-4157. [CrossRef]

574. Yang, H.F.; Shan, C.S.; Li, F.H.; Han, D.X.; Zhang, Q.X.; Niu, L. Covalent functionalization of polydisperse chemically-converted graphene sheets with amine-terminated ionic liquid. Chem. Commun. 2009, 3880-3882. [CrossRef] [PubMed]

575. Su, Q.; Pang, S.P.; Alijani, V.; Li, C.; Feng, X.L.; Mullen, K. Composites of Graphene with Large Aromatic Molecules. Adv. Mater. 2009, 21, 3191-3195. [CrossRef]

576. Tan, L.; Zhou, K.G.; Zhang, Y.H.; Wang, H.X.; Wang, X.D.; Guo, Y.F.; Zhang, H.L. Nanomolar detection of dopamine in the presence of ascorbic acid at $\beta$-cyclodextrin/graphene nanocomposite platform. Electrochem. Commun. 2010, 12, 557-560. [CrossRef]

577. Lomeda, J.R.; Doyle, C.D.; Kosynkin, D.V.; Hwang, W.F.; Tour, J.M. Diazonium Functionalization of Surfactant-Wrapped Chemically Converted Graphene Sheets. J. Am. Chem. Soc. 2008, 130, 16201-16206. [CrossRef] [PubMed]

578. Layek, R.K.; Nandi, A.K. A review on synthesis and properties of polymer functionalized graphene. Polymer 2013, 54, 5087-5103. [CrossRef]

579. Elias, D.C. Control of graphene's properties by reversible hydrogenation: Evidence for graphane. Science 2009, 323, 610-613. [CrossRef]

580. Zheng, L.; Li, Z.; Bourdo, S.; Watanabe, F.; Ryersonb, C.C.; Biris, A.S. Catalytic hydrogenation of graphene films. Chem. Commun. 2011, 47, 1213-1215. [CrossRef]

581. Schafer, R.A.; Englert, J.M.; Wehrfritz, P.; Bauer, W.; Hauke, F.; Seyller, T.; Hirsh, A. On the way to graphane-pronounced fluorescence of polyhydrogenated graphene. Angew. Chem. Int. Ed. 2013, 52, 754-757. [CrossRef]

582. Karlicky, F.; Datta, K.K.R.; Otyepka, M.; Zboril, R. Halogenated Graphenes: Rapidly Growing Family of Graphene Derivatives. ACS Nano 2013, 7, 6434-6464. [CrossRef]

583. Sinitskii, A.; Dimiev, A.; Corley, D.A.; Fursina, A.A.; Kosynkin, D.V.; Tour, J.M. Kinetics of diazonium functionalization of chemically converted graphene nanoribbons. ACS Nano 2010, 4, 1949-1954. [CrossRef]

584. Fang, M.; Wang, K.; Lu, H.; Yang, Y.; Nutt, S. Covalent polymer functionalization of graphene nanosheets and mechanical properties of composites. J. Mater. Chem. 2009, 19, 7098-7105. [CrossRef]

585. Lim, H.; Lee, J.S.; Shin, H.J.; Shin, H.S.; Choi, H.C. Spatially resolved spontaneous reactivity of diazonium salt on edge and basal plane of graphene without surfactant and its doping effect. Langmuir 2010, 26, 12278-12284.

586. Bekyarova, E.; Itkis, M.; Ramesh, P.; Berger, C.; Sprinkle, M.; deHeer, W.A.; Haddon, R.C. Chemical modification of epitaxial graphene: Spontaneous grafting of aryl groups. J. Am. Chem. Soc. 2009, 131, 1336-1337. [CrossRef] [PubMed]

587. Jin, Z.; McNicholas, T.P.; Shih, C.J.; Wang, Q.H.; Paulus, G.L.; Hilmer, A.J.; Shimizu, S.; Strano, M.S. Click chemistry on solution-dispersed graphene and monolayer CVD graphene. Chem. Mater. 2011, 23, 3362-3370.

588. Qintana, M.; Spyrou, K.; Grzelczak, M.; Browne, W.R.; Rudolf, P.; Prato, M. Functionalization of graphene via 1,3-dipolar cycloaddition. ACS Nano 2010, 4, 3527-3533. [CrossRef] [PubMed]

589. Georgakilas, V.; Bourlinos, A.B.; Zboril, R.; Steriotis, T.A.; Dallas, P.; Stubos, A.K.; Trapalis, C. Organic functionalisation of graphenes. Chem. Commun. 2010, 46, 1766-1768.

590. Ragoussi, M.E.; Malig, J.; Katsukis, G.; Butz, B.; Spiecker, E.; De La Torre, G.; Torres, T.; Guldi, D.M. Linking photo- and redoxactive phthalocyanines covalently to graphene. Angew. Chem. Int. Ed. 2012, 51, 6421-6425. [CrossRef] [PubMed]

591. Liu, L.H.; Lerner, M.M.; Yan, M. Derivitization of pristine graphene with well-defined chemical functionalities. Nano Lett. 2010, 10, 3754-3756. [CrossRef] [PubMed]

592. Vadukumpully, S.; Gupta, J.; Zhang, Y.; Xu, G.Q.; Valiyaveettil, S. Functionalization of surfactant wrapped graphene nanosheets with alkylazides for enhanced dispersibility. Nanoscale 2011, 3, 303-308. [CrossRef] [PubMed]

593. Hirsch, A.; Brettreich, M. Fullerenes-Chemistry and Reactions; Wiley-VCH: Weinheim, Germany, 2005.

594. Economopoulos, S.P.; Rotas, G.; Miyata, Y.; Shinohara, H.; Tagmatarchis, N. Exfoliation and chemical modification using microwave irradiation affording highly functionalized graphene. ACS Nano 2010, 4, 7499-7507. [CrossRef]

595. Xu, Y.; Bai, H.; Lu, G.; Li, C.; Shi, G.Q. Flexible graphene films via the filtration of water-soluble noncovalent functionalized graphene sheets. J. Am. Chem. Soc. 2008, 130, 5856-5857. [CrossRef] [PubMed]

596. Wang, Y.; Chen, X.; Zhong, Y.; Zhu, F.; Loh, K.P. Large area, continuous, few-layered graphene as anodes in organic photovoltaic devices. Appl. Phys. Lett. 2009, 95, 063302. [CrossRef]

597. An, X.; Butler, T.W.; Washington, M.; Nayak, S.K.; Kar, S. Optical and sensing properties of 1-pyrenecarboxylic acid-functionalized graphene films laminated on polydimethylsiloxane membranes. ACS Nano 2011, 5, 1003-1011. [CrossRef]

598. Georgakilas, V.; Tiwari, J.N.; Kemp, K.C.; Perman, J.A.; Bourlinos, A.B.; Kwang, S.K.; Zboril, R. Noncovalent Functionalization of Graphene and Graphene Oxide for Energy Materials, Biosensing, Catalytic, and Biomedical Applications. Chem. Rev. 2016, 116, 5464-5519. [CrossRef]

599. Xu, Y.; Zhao, L.; Bai, H.; Hong, W.; Li, C.; Shi, G. Chemically Converted Graphene Induced Molecular Flattening of 5,10,15,20Tetrakis(1-methyl-4-pyridinio)porphyrin and Its Application for Optical Detection of Cadmium(II) Ions. J. Am. Chem. Soc. 2009, 131, 13490-13497. [CrossRef] 
600. Qu, S.; Li, M.; Xie, L.; Huang, X.; Yang, J.; Wang, N.; Yang, S. Noncovalent Functionalization of Graphene Attaching [6,6]-PhenylC61-butyric Acid Methyl Ester (PCBM) and Application as Electron Extraction Layer of Polymer Solar Cells. ACS Nano 2013, 7, 4070-4081. [CrossRef] [PubMed]

601. Wahid, M.H.; Stroeher, U.H.; Eroglu, E.; Chen, X.; Vimalanathan, K.; Raston, C.L.; Boulos, R.A. Aqueous Based Synthesis of Antimicrobial-Decorated Graphene. J. Coll. Interf. Sci. 2015, 443, 88-96. [CrossRef] [PubMed]

602. Si, C.; Sun, Z.; Liu, F. Strain engineering of graphene: A review. Nanoscale 2016, 8, 3207-3217. [CrossRef] [PubMed]

603. Choi, S.-M.; Jhi, S.-H.; Son, Y.-W. Effects of strain on electronic properties of graphene. Phys. Rev. B 2010, 81, 081407. [CrossRef]

604. Huang, M.; Yan, H.; Chen, C.; Song, T.; Heinz, T.F.; Hone, J. Phonon softening and crystallographic orientation of strained graphene studied by Raman spectroscopy. Proc. Natl. Acad. Sci. USA 2009, 106, 7304-7308. [CrossRef] [PubMed]

605. Mohiuddin, T.M.G.; Lombardo, A.; Nair, R.R.; Bonetti, A.; Savini, G.; Jalil, R.; Bonini, N.; Basko, D.M.; Galiotis, C.; Marzari, N.; et al. Uniaxial strain in graphene by Raman spectroscopy: G peak splitting, Grüneisen parameters, and sample orientation. Phys. Rev. B 2009, 79, 205433. [CrossRef]

606. Boland, C.S.; Khan, U.; Backes, C.; O’Neil, A.; McCauley, J.; Duane, S.; Shanker, R.; Liu, Y.; Jurewicz, I.; Dalton, A.B.; et al. Sensitive, high-strain, high-rate bodily motion sensors based on graphene-rubber composites. ACS Nano 2014, 8, 8819-8830. [CrossRef]

607. Hempel, M.; Nezich, D.; Kong, J.; Hofman, M. A Novel Class of Strain Gauges Based on Layered Percolative Films of 2D Materials. Nano Lett. 2012, 12, 5714-5718. [CrossRef]

608. Bae, S.-H.; Lee, Y.; Sharma, B.K.; Lee, H.-J.; Kim, J.-H.; Ahn, J.-H. Graphene-based transparent strain sensor. Carbon 2013, 51, 236-242. [CrossRef]

609. Tian, H.; Shu, Y.; Cui, Y.-L.; Mi, W.-T.; Yang, Y.; Xie, D.; Ren, T.-L. Scalable fabrication of high-performance and flexible graphene strain sensors. Nanoscale 2014, 6, 669-705. [CrossRef] [PubMed]

610. Qin, Y.; Peng, Q.; Ding, Y.; Lin, Z.; Wang, C.; Li, Y.; Xu, F.; Li, J.; Yuan, Y.; He, X.; et al. Lightweight, superelastic, and mechanically flexible graphene/polyimide nanocomposite foam for strain sensor application. ACS Nano 2015, 9, 8933-8941. [CrossRef]

611. Yu, R.; Zhu, C.; Wan, J.; Li, Y.; Hong, X. Review of Graphene-Based Textile Strain Sensors, with Emphasis on Structure Activity Relationship. Polymers 2021, 13, 151. [CrossRef]

612. Zhou, M.; Zhai, Y.M.; Dong, S.J. Electrochemical Sensing and Biosensing Platform Based on Chemically Reduced Graphene Oxide. Anal. Chem. 2009, 81, 5603-5613. [CrossRef] [PubMed]

613. McCreery, R.L. Advanced Carbon Electrode Materials for Molecular Electrochemistry. Chem. Rev. 2008, 108, 2646-2687. [CrossRef]

614. Qi, M.; Zhang, Y.; Cao, C.; Luc, Y.; Liu, G. Increased sensitivity of extracellular glucose monitoring based on AuNP decorated GO nanocomposites. RSC Adv. 2016, 6, 39180-39187. [CrossRef]

615. Cui, M.; Xu, B.; Hu, C.; Shao, H.B.; Qu, L. Direct electrochemistry and electrocatalysis of glucose oxidase on three-dimensional interpenetrating, porous graphene modified electrode. Electrochim. Acta 2013, 98, 48-53. [CrossRef]

616. Liang, B.; Guo, X.; Fang, L.; Hu, Y.; Yang, G.; Zhu, Q.; Wei, J.; Ye, X. Study of Direct Electron Transfer and Enzyme Activity of Glucose Oxidase on Graphene Surface. Electrochem. Commun. 2015, 50, 1-5. [CrossRef]

617. Wang, Z.; Zhou, X.; Zhang, J.; Boey, F.; Zhang, H. Direct Electrochemical Reduction of Single-Layer Graphene Oxide and Subsequent Functionalization with Glucose Oxidase. J. Phys. Chem. C 2009, 113, 14071-14075. [CrossRef]

618. Liu, J.; Kong, N.; Li, A.; Luo, X.; Cui, L.; Wang, R.; Feng, S. Graphene Bridged Enzyme Electrodes for Glucose Biosensing Application. Analyst 2013, 138, 2567-2575. [CrossRef] [PubMed]

619. Jin, L.; Yang, K.; Yao, K.; Zhang, S.; Tao, H.; Lee, S.-T.; Peng, R. Functionalized Graphene Oxide in Enzyme Engineering: A Selective Modulator for Enzyme Activity and Thermostability. ACS Nano 2012, 6, 4864-4875. [CrossRef] [PubMed]

620. Shan, C.; Yang, H.; Song, J.; Han, D.; Ivaska, A.; Niu, L. Direct Electrochemistry of Glucose Oxidase and Biosensing for Glucose Based on Graphene. Anal. Chem. 2009, 81, 2378-2382. [CrossRef]

621. Quian, L.; Lu, L. Three Dimensional Porous Graphene-Chitosan Composites from Ice-Induced Assembly for Direct Electron Transfer and Electrocatalysis of Glucose Oxidase. RSC Adv. 2014, 4, 38273-38280. [CrossRef]

622. Deepalakshmi, T.; Tran, D.T.; Kim, N.H.; Chong, K.T.; Lee, J.H. Nitrogen-Doped Graphene-Encapsulated Nickel Cobalt Nitride as a Highly Sensitive and Selective Electrode for Glucose and Hydrogen Peroxide Sensing Applications. ACS Appl. Mater. Interf. 2018, 10, 35847-35858. [CrossRef]

623. Jiang, D.; Liu, Q.; Wang, K.; Qian, J.; Dong, X.; Yang, Z.; Du, X.; Qiu, B. Enhanced Non-Enzymatic Glucose Sensing Based on Copper Nanoparticles Decorated Nitrogen-Doped Graphene. Biosens. Bioelectron. 2014, 54, 273-278. [CrossRef]

624. Hoa, L.T.; Sun, K.G.; Hur, H. Highly Sensitive Non- Enzymatic Glucose Sensor Based on Pt Nanoparticle Decorated Graphene Oxide Hydrogel. Sens. Actuators B 2015, 210, 618-623. [CrossRef]

625. Gao, H.; Xiao, F.; Ching, C.B.; Duan, H. One-Step Electrochemical Synthesis of PtNi Nanoparticle-Graphene Nanocomposites for Nonenzymatic Amperometric Glucose Detection. ACS Appl. Mater. Interf. 2011, 3, 3049-3057. [CrossRef] [PubMed]

626. Dey, R.S. Redox-Functionalized Graphene Oxide Architecture for the Development of Amperometric Biosensing Platform. ACS Appl. Mater. Interf. 2013, 5, 4791-4798. [CrossRef] [PubMed]

627. Ruecha, N.; Rangkupan, R.; Rodthongkum, N.; Chailapakul, O. Novel Paper-Based Cholesterol Biosensor Using Graphene/Polyvinylpyrrolidone/Polyaniline Nanocomposite. Biosens. Bioelectron. 2014, 52, 13-19. [CrossRef] [PubMed]

628. Dey, R.S.; Raj, C.R. Development of an Amperometric Cholesterol Biosensor Based on Graphene-Pt Nanoparticle Hybrid Material. J. Phys. Chem. C 2010, 114, 21427-21433. [CrossRef] 
629. Dey, R.S.; Raj, C.R. Enzyme-integrated cholesterol biosensing scaffold based on in situ synthesized reduced graphene oxide and dendritic Pd nanostructure. Biosens. Bioelectron. 2014, 62, 357-364. [CrossRef] [PubMed]

630. Cao, S.; Zhang, L.; Chai, Y.; Yuan, R. Electrochemistry of Cholesterol Biosensor Based on a Novel Pt-Pd Bimetallic Nanoparticle Decorated Graphene Catalyst. Talanta 2013, 109, 167-172. [CrossRef]

631. Lakshmi, B.V.S.; Sharma, A.; Solanki, P.R.; Avasthi, K. Mesoporous Polyaniline Nanofiber Decorated Graphene Micro-Flowers for Enzyme-Less Cholesterol Biosensors. Nanotechnology 2016, 27, 345101. [CrossRef]

632. Rengaraj, A.; Haldorai, Y.; Kwak, C.H.; Ahn, S.; Jeon, K.-J.; Park, S.H.; Han, Y.-K.; Huh, Y.S. Electrodeposition of Flower-Like Nickel Oxide on CVD-Grown Graphene to Develop an Electrochemical Non-Enzymatic Biosensor. J. Mater. Chem. B 2015, 3 , 6301-6309. [CrossRef]

633. Chen, W.; Cai, S.; Ren, Q.-Q.; Wen, W.; Zhao, Y.-D. Recent Advances in Electrochemical Sensing for Hydrogen Peroxide: A Review. Analyst 2012, 137, 49-58. [CrossRef]

634. Hou, B.; Liu, H.; Qi, S.; Zhu, Y.; Zhou, B.; Jiang, X.; Zhu, L. Preparation of Pristine Graphene in Ethanol Assisted by Organic Salts for Nonenzymatic Detection of Hydrogen Peroxide. J. Coll. Interf. Sci. 2018, 510, 103-110. [CrossRef] [PubMed]

635. Guo, S.; Wen, D.; Zhai, Y.; Dong, S.; Wang, E. Platinum Nanoparticle Ensemble-on-Graphene Hybrid Nanosheet: One-Pot, Rapid Synthesis, and Used as New Electrode Material for Electrochemical Sensing. ACS Nano 2010, 4, 3959-3968. [CrossRef]

636. Zhan, B.; Liu, H.; Li, C.; Wang, L.; Huang, W.; Dong, X. A Hydrogen Peroxide Electrochemical Sensor Based on Silver Nanoparticles Decorated Three- Dimensional Graphene. Appl. Phys. Lett. 2014, 104, 243704. [CrossRef]

637. Yu, B.; Feng, J.; Liu, S.; Zhang, T. Preparation of Reduced Graphene Oxide Decorated with High Density Ag Nanorods for Non-Enzymatic Hydrogen Peroxide Detection. RSC Adv. 2013, 3, 14303-14307. [CrossRef]

638. Zhang, M.; Wang, Z. Nanostructured Silver Nanowires-Graphene Hybrids for Enhanced Electrochemical Detection of Hydrogen Peroxide. Appl. Phys. Lett 2013, 102, 213104. [CrossRef]

639. Xi, Q.; Chen, X.; Evans, D.G.; Yang, W. Gold Nanoparticle-Embedded Porous Graphene Thin Films Fabricated via Layer-by-Layer Self-Assembly and Subsequent Thermal Annealing for Electrochemical Sensing. Langmuir 2012, 28, 9885-9892. [CrossRef] [PubMed]

640. Chen, D.; Zhuang, X.; Zhai, J.; Zheng, Y.; Lu, H.; Chen, L. Preparation of Highly Sensitive Pt Nanoparticles-Carbon Quantum Dots/Ionic Liquid Functionalized Graphene Oxide Nanocomposites and Application for $\mathrm{H}_{2} \mathrm{O}_{2}$ Detection. Sens. Actuator B 2018, 255, 1500-1506. [CrossRef]

641. Sun, Y.; Luo, M.; Qin, Y.; Zhu, S.; Li, Y.; Xu, N.; Meng, X.; Ren, Q.; Wang, L.; Guo, S. Atomic-Thick PtNi Nanowires Assembled on Graphene for High-Sensitivity Extracellular Hydrogen Peroxide Sensors. ACS Appl. Mater. Interf. 2017, 9, 34715-34721. [CrossRef] [PubMed]

642. Cheng, C.; Zhang, C.; Gao, X.; Zhuang, Z.; Du, C.; Chen, W. 3D Network and 2D Paper of Reduced Graphene Oxide/Cu2O Composite for Electrochemical Sensing of Hydrogen Peroxide. Anal. Chem. 2018, 90, 1983-1991. [CrossRef]

643. Nagarajan, R.D.; Murugan, P.; Sundramoorthy, A.K. Selective Electrochemical Sensing of NADH and NAD+ Using Graphene/Tungstate Nanocomposite Modified Electrode. ChemistrySelect 2020, 5, 14643-14651. [CrossRef]

644. Gao, F.; Cai, X.; Wang, X.; Gao, C.; Liu, S.; Gao, F.; Wang, Q. Highly Sensitive and Selective Detection of Dopamine in the Presence of Ascorbic Acid at Graphene Oxide Modified Electrode. Sens. Actuators B 2013, 186, 380-387. [CrossRef]

645. Tian, X.; Cheng, C.; Yuan, H.; Du, J.; Xiao, D.; Xie, S.; Choi, M.M.F. Simultaneous Determination of L-Ascorbic Acid, Dopamine and Uric Acid With Gold Nanoparticles- $\beta$-Cyclodextrin-Graphene-Modified Electrode by Square Wave Voltammetry. Talanta 2012, 93, 79-85. [CrossRef]

646. Ramakrishnan, S.; Pradeep, K.R.; Raghul, A.; Senthilkumar, R.; Rangarajan, M.; Kothurkar, N.K. One-Step Synthesis of PtDecorated Graphene-Carbon Nanotubes for the Electrochemical Sensing of Dopamine, Uric Acid and Ascorbic Acid. Anal. Methods 2015, 7, 779-786. [CrossRef]

647. Li, S.-M.; Wang, Y.-S.; Hsiao, S.-T.; Liao, W.-H.; Lin, C.-W.; Yang, S.-Y.; Tien, H.-W.; Ma, C.-C.M.; Hu, C.-C. Fabrication of a Silver Nanowire-Reduced Graphene Oxide-Based Electrochemical Biosensor and its Enhanced Sensitivity in the Simultaneous Determination of Ascorbic Acid, Dopamine, and Uric Acid. J. Mater. Chem. C 2015, 3, 9444-9453. [CrossRef]

648. Wang, X.; Wu, M.; Tang, W.; Zhu, Y.; Wang, L.; Wang, Q.; He, P.; Fang, Y. Simultaneous Electrochemical Determination of Ascorbic Acid, Dopamine and Uric Acid Using a Palladium Nanoparticle/Graphene/Chitosan Modified Electrode. J. Electroanal. Chem. 2013, 695, 10-16. [CrossRef]

649. Li, S.; Ma, Y.; Liu, Y.; Xin, G.; Wang, M.; Zhang, Z.; Liu, Z. Electrochemical sensor based on a three dimensional nanostructured MoS 2 nanosphere-PANI/reduced graphene oxide composite for simultaneous detection of ascorbic acid, dopamine, and uric acid. RSC Adv. 2019, 9, 2997-3003. [CrossRef]

650. Yue, H.Y.; Huang, S.; Chang, J.; Heo, C.; Yao, F.; Adhikari, S.; Huy, T.Q.; Luan, N.V.; Lee, Y.H. ZnO Nanowire Arrays on 3D Hierachical Graphene Foam: Biomarker Detection of Parkinson's Disease. ACS Nano 2014, 8, 1639-1646. [CrossRef]

651. Dutta, D.; Chandra, S.; Swain, A.K.; Bahadur, D. $\mathrm{SnO}_{2}$ Quantum Dots-Reduced Graphene Oxide Composite for Enzyme-Free Ultrasensitive Electrochemical Detection of Urea. Anal. Chem. 2014, 86, 5914-5921. [CrossRef] [PubMed]

652. Du, Y.; Dong, S. Nucleic Acid Biosensors: Recent Advances and Perspectives. Anal. Chem. 2017, 89, 189-215. [CrossRef] [PubMed]

653. Zhu, X.; Sun, L.; Chen, Y.; Ye, Z.; Shen, Z.; Li, G. Combination of Cascade Chemical Reactions with Graphene-DNA Interaction to Develop New Strategy for Biosensor Fabrication. Biosens. Bioelectron. 2013, 47, 32-37. [CrossRef] 
654. Wang, J.; Shi, A.; Fang, X.; Han, X.; Zhang, Y. An ultrasensitive super sandwich electrochemical DNA biosensor based on gold nanoparticles decorated reduced graphene oxide. Anal. Biochem. 2015, 469, 71-75. [CrossRef] [PubMed]

655. Huang, H.; Bai, W.; Dong, C.; Guo, R.; Liu, Z. An ultrasensitive Electrochemical DNA Biosensor Based on Graphene/Au Nanorod/Polythionine for Human Papillomavirus DNA Detection. Biosens. Bioelectron. 2015, 68, 442-446. [CrossRef]

656. Yang, T.; Chen, H.; Qiu, Z.; Yu, R.; Luo, S.; Li, W.; Jiao, K. Direct Electrochemical Vibrio DNA Sensing Adopting Highly Stable Graphene-Flavin Mononucleotide Aqueous Dispersion Modified Interface. ACS Appl. Mater. Interf. 2018, 10, 4540-4547. [CrossRef] [PubMed]

657. Singh, A.; Sinsinbar, G.; Choudhary, M.; Kumar, V.; Pasricha, R.; Verma, H.N.; Singh, S.P.; Arora, K. Graphene Oxide-Chitosan Nanocomposite Based Electrochemical DNA Biosensor for Detection of Typhoid. Sens. Actuator B 2013, 185, 675-684. [CrossRef]

658. Peng, H.-P.; Hu, Y.; Liu, P.; Deng, Y.-N.; Wang, P.; Chen, W.; Liu, A.-L.; Chen, Y.-Z.; Lin, X.-H. Label-Free Electrochemical DNA Biosensor for Rapid Detection of Mutidrug Resistance Gene Based on Au Nanoparticles/Toluidine Blue-Graphene Oxide Nanocomposites. Sens. Actuator B 2015, 207, 269-276. [CrossRef]

659. Han, X.; Fang, X.; Shi, A.; Wang, J.; Zhang, Y. An Electrochemical DNA Biosensor Based on Gold Nanorods Decorated Graphene Oxide Sheets for Sensing Platform. Anal. Biochem. 2013, 443, 117-123. [CrossRef]

660. Wang, Y.; Bai, X.; Wen, W.; Zhang, X.; Wang, S. Ultrasensitive Electrochemical Biosensor for HIV Gene Detection Based on Graphene Stabilized Gold Nanoclusters with Exonuclease Amplification. ACS Appl. Mater. Interf. 2015, 7, 18872-18879. [CrossRef]

661. Hajian, R.; Balderston, S.; Tran, T.; deBoer, T.; Etienne, J.; Sandhu, M.; Wauford, N.A.; Chung, J.-Y.; Nokes, J.; Athaiya, M.; et al Detection of unamplified target genes via CRISPR-Cas9 immobilized on a graphene field-effect transistor. Nat. Biomed. Eng. 2019, 3, 427-437. [CrossRef]

662. Gao, Z.; Xia, H.; Zauberfman, J.; Tomaiuolo, M.; Ping, J.; Zhang, Q.; Ducos, P.; Ye, H.; Wang, S.; Yang, X.; et al. Detection of Sub-fM DNA with Target Recycling and Self-Assembly Amplification on Graphene Field-Effect Biosensors. Nano Lett. 2018, 18, 3509-3515. [CrossRef]

663. Lin, D.; Wu, J.; Wang, M.; Yan, F.; Ju, H. Triple Signal Amplification of Graphene Film, Polybead Carried Gold Nanoparticles as Tracing Tag and Silver Deposition for Ultrasensitive Electrochemical Immunosensing. Anal. Chem. 2012, 84, 3662-3668. [CrossRef]

664. Ali, M.A.; Singh, C.; Srivastava, S.; Admane, P.; Agrawal, V.V.; Sumana, G.; John, R.; Panda, A.; Dong, L.; Malhotra, B.D. Graphene Oxide-Metal Nanocomposites for Cancer Biomarker Detection. RSC Adv. 2017, 7, 35982-35991. [CrossRef]

665. Rajaji, U.; Muthumariyappan, A.; Chen, S.-M.; Chen, T.-W.; Ramalingam, R.J. A novel electrochemical sensor for the detection of oxidative stress and cancer biomarker (4-nitroquinoline $\mathrm{N}$-oxide) based on iron nitride nanoparticles with multilayer reduced graphene nanosheets modified electrode. Sens. Actuators B 2019, 291, 120-129. [CrossRef]

666. Li, R.; Fangchao, C.; Cui, F.; Zhu, H.; Sun, X.; Li, Z. Electrochemical sensor for detection of cancer cell based on folic acid and octadecylamine-functionalized graphene aerogel microspheres. Biosens. Bioelectron. 2018, 119, 156-162.

667. Shahzad, F.; Zaidi, S.A.; Koo, C.M. Highly sensitive electrochemical sensor based on environmentally friendly biomass-derived sulfur-doped graphene for cancerbiomarker detection. Sens. Actuators B 2017, 241, 716-724. [CrossRef]

668. Zeng, Y.; Bao, J.J.; Zhao, Y.; Huo, D.; Chen, M.; Yang, M.; Fa, H.; Hou, C. A sensitive label-free electrochemical immunosensor for detection of cytokeratin 19 fragment antigen 21-1 based on 3D graphene with gold nanopaticle modified electrode. Talanta 2018, 178, 122-128. [CrossRef] [PubMed]

669. Khoshroo, A.; Mazloum-Ardakani, M.; Forat-Yazdi, M. Enhanced performance of label-free electrochemical immunosensor for carbohydrate antigen 15-3 based on catalytic activity of cobalt sulfide/graphene nanocomposite. Sens. Actuators B 2018, 255, 580-587. [CrossRef]

670. Kumar, V.; Srivastava, S.; Umrao, S.; Kumar, R.; Nath, G.; Sumana, G.; Saxena, P.S.; Srivastava, A. Nanostructured PalladiumReduced Graphene Oxide Platform for High Sensitive, Label Free Detection of a Cancer Biomarker. RSC Adv. 2014, 4, $2267-2273$. [CrossRef]

671. Kumar, S.; Sharma, J.G.; Maji, S.; Malhotra, B.D. Nanostructured Zirconia Decorated Reduced Graphene Oxide Based Efficient Biosensing Platform for Non-Invasive Oral Cancer Detection. Biosens. Bioelectron. 2016, 78, 497-504. [CrossRef]

672. Sharafeldin, M.; Bishop, G.; Bhakta, S.; El-Sawy, A.; Suib, S.L.; Rusling, J.F. $\mathrm{Fe}_{3} \mathrm{O}_{4}$ Nanoparticles on Graphene Oxide Sheets for Isolation and Ultrasensitive Amperometric Detection of Cancer Biomarker Proteins. Biosens. Bioelectron. 2017, 91, 359-366. [CrossRef]

673. Lu, Y.; Liang, X.; Niyungeko, C.; Zhou, J.; Xu, J.; Tian, G. A review of the identification and detection of heavy metal ions in the environment by voltammetry. Talanta 2018, 178, 324-338. [CrossRef]

674. Liu, X.; Yao, Y.; Ying, Y.; Ping, J. Recent advances in nanomaterial-enabled screen-printed electrochemical sensors for heavy metal detection. Trends Anal. Chem. 2019, 115, 187-202. [CrossRef]

675. Ullah, N.; Mansha, M.; Khan, I.; Qurashi, A. Nanomaterial-based optical chemical sensors for the detection of heavy metals in water: Recent advances and challenges. Trends Anal. Chem. 2018, 100, 155-166. [CrossRef]

676. Zhang, L.; Peng, D.; Liang, R.P.; Qiu, J.D. Graphene-based optical nanosensors for detection of heavy metal ions. Trends Anal. Chem. 2018, 102, 280-289. [CrossRef]

677. Perez-Fernendez, B.; Costa-Garcia, A.; de la Escosura-Muniz, A. Electrochemical (Bio)Sensors for Pesticides Detection Using Screen-Printed Electrodes. Biosensors 2020, 10, 32. [CrossRef]

678. Singh, M.; Bhardiya, S.R.; Verma, F.; Rai, V.K.; Rai, A. Graphene-based Nanomaterials for Fabrication of "Pesticide" Electrochemical Sensors. Curr. Graphene Sci. 2020, 3, 26-40. [CrossRef] 
679. Facure, M.H.M.; Mercante, L.A.; Mattoso, L.H.C.; Correa, D.S. Detection of trace levels of organophosphate pesticides using an electronic tongue based on graphene hybrid nanocomposites. Talanta 2017, 167, 59-66. [CrossRef] [PubMed]

680. Li, G.; Xia, Y.; Tian, Y.; Wu, Y.; Liu, J.; He, Q.; Chen, D. Review-recent developments on -based electrochemical sensors toward nitrite. J. Electrochem. Soc. 2019, 166, B881-B895. [CrossRef]

681. Zhu, G.; Sun, H.; Zou, B.; Liu, Z.; Sun, N.; Yi, Y.; Wong, K. Electrochemical sensing of 4-nitrochlorobenzene based on carbon nanohorns/graphene oxide nanohybrids. Biosens. Bioelectron. 2018, 106, 136-141. [CrossRef]

682. Wang, C.; Zhang, L.; Guo, Z.H.; Xu, J.G.; Wang, H.Y.; Xhai, K.F.; Zhuo, X. A novel hydrazine electrochemical sensor based on the high specific surface area graphene. Microchim. Acta 2010, 169, 1-6. [CrossRef]

683. Qi, P.; Wan, Y.; Zhang, D.; Wu, J. Reduced Graphene Sheets Modified Electrodes for Electrochemical Detection of Sulfide. Electroanalysis 2011, 23, 2796-2801. [CrossRef]

684. Fan, Y.; Liu, J.H.; Yang, C.P.; Yu, M.; Liu, P. Graphene-polyaniline composite film modified electrode for voltammetric determination of 4-aminophenol. Sens. Actuators B 2011, 157, 669-674. [CrossRef]

685. Cao, L.Y.; Sun, H.M.; Li, J.; Lu, L.H. An enhanced electrochemical platform based on graphene-polyoxometalate nanomaterials for sensitive determination of diphenolic compounds. Anal. Methods 2011, 3, 1587-1594. [CrossRef]

686. Li, S.J.; Xing, Y.; Wang, G.F. A graphene-based electrochemical sensor for sensitive and selective determination of hydroquinone. Microchim. Acta 2012, 176, 163-168. [CrossRef]

687. Meng, F.-L.; Guo, Z.; Huang, X.-J. Graphene-based hybrids for chemiresistive gas sensors. Trends Anal. Chem. 2015, 68, 37-47. [CrossRef]

688. Vedala, H.; Sorescu, D.C.; Kotchey, G.P.; Star, A. Chemical sensitivity of graphene edges decorated with metal nanoparticles. Nano Lett. 2011, 11, 2342-2347. [CrossRef] [PubMed]

689. Zhang, D.; Jiang, C.; Liu, J.; Cao, Y. Carbon monoxide gas sensing at room temperature using copper oxide-decorated graphene hybrid nanocomposite prepared by layer-by-layer self-assembly. Sens. Actuatos B 2017, 247, 875-882. [CrossRef]

690. Yoon, H.J.; Jun, D.H.; Zhou, Z.; Yang, S.S.; Cheng, M.C.-C. Carbon dioxide gas sensor using a graphene sheet. Sens. Actuators B 2011, 157, 310-313. [CrossRef]

691. Xu, B.; Huang, J.; Xu, X.; Zhou, A.; Ding, L. Ultrasensitive NO Gas Sensor Based on the Graphene Oxide-Coated Long-Period Fiber Grating. ACS Appl. Mater. Interf. 2019, 11, 40868-40874. [CrossRef] [PubMed]

692. Fei, H.; Wu, G.; Cheng, W.-Y.; Yan, W.; Xu, H.; Zhang, D.; Zhang, D.; Zhao, Y.; Lv, Y.; Chen, Y.; et al. Enhanced NO2 Sensing at Room Temperature with Graphene via Monodisperse Polystyrene Bead Decoration. ACS Omega 2019, 4, 3812-3819. [CrossRef] [PubMed]

693. Kumar, R.; Kaur, A. Chemiresistive gas sensors based on thermally reduced graphene oxide for sensing sulphur dioxide at room temperature. Diam. Relat. Mater. 2020, 109, 108039. [CrossRef]

694. Ly, T.N.; Park, S. Highly sensitive ammonia sensor for diagnostic purpose using reduced graphene oxide and conductive polymer. Sci. Rep. 2018, 8, 18030. [CrossRef]

695. Vikrant, K.; Kumar, V.; Kim, K.-H. Graphene materials as a superior platform for advanced sensing strategies against gaseous ammonia. J. Mater. Chem. A 2018, 6, 22391-22410. [CrossRef]

696. Gupta, C.S.; Shyamasree, C.; Chatterjee, S.; Roy, A.K.; Chakraborty, A.K. Graphene-metal oxide nanohybrids for toxic gas sensor: A review. Sens. Actuators B 2015, 221, 1170-1181. [CrossRef]

697. Xie, Y.L.; Zhao, S.Q.; Ye, H.L.; Yuan, J.; Song, P.; Hu, S.Q. Graphene $/ \mathrm{CeO}_{2}$ hybrid materials for the simultaneous electrochemical detection of cadmium(II), lead(II),copper(II), and mercury(II). J. Electroanal. Chem. 2015, 757, 235-242. [CrossRef]

698. Lee, R.M.; Chen, Z.; Li, L.; Liu, E. Reduced graphene oxide decorated with tin nanoparticles through electrodeposition for simultaneous determination of trace heavy metals. Electreocim. Acta 2015, 174, 207-214. [CrossRef]

699. Lee, S.; Oh, J.; Kim, D.; Piao, Y. A sensitive electrochemical sensor using an iron oxide/graphene composite for the simultaneous detection of heavy metal ions. Talanta 2016, 160, 528-536. [CrossRef]

700. Gumpu, M.B.; Veerapandian, M.; Krishnan, U.M.; Rayappan, J.B. Simultaneous electrochemical detection of Cd(II), Pb(II), As(III) and $\mathrm{Hg}$ (II) ions using ruthenium(II)-textured graphene oxide nanocomposite. Talanta 2017, 162, 574-582. [CrossRef]

701. Ruecha, N.; Rodthongkum, N.; Cate, D.M.; Volkens, J.; Chailapakul, O.; Henry, C.S. Sensitive electrochemical sensor using a graphene-polyaniline nanocomposite for simultaneous detection of $\mathrm{Zn}(\mathrm{II}), \mathrm{Cd}(\mathrm{II})$, and $\mathrm{Pb}(\mathrm{II})$. Anal. Chim. Acta 2015, 874, 40-48. [CrossRef]

702. Krishnan, S.K.; Singh, E.; Singh, P.; Meyyappan, M.; Singh Nalwa, H. A review on graphene-based nanocomposites for electrochemical and fluorescent biosensors. RSC Adv. 2019, 9, 8778-8881. [CrossRef]

703. Jin, X.; Feng, C.; Ponnamma, D.; Yi, Z.; Parameswaranpillai, J.; Sabu, T.; Salim, N.V. Review on exploration of graphene in the design and engineering of smart sensors, actuators and soft robotics. Chem. Eng. J. Adv. 2020, 4, 100034. [CrossRef]

704. Zheng, P.; Wu, N. Fluorescence and Sensing Applications of Graphene Oxide and Graphene Quantum Dots: A Review. Chem. Asian J. 2017, 12, 2343-2353. [CrossRef] [PubMed] 